\title{
A Novel Intrabody Communication Transceiver for Biomedical
}

\section{Applications}

\author{
Mir Hojjat Seyedi \\ B.Sc. (Telecommunications), M.Sc. (Electronics)
}

College of Engineering and Science

Victoria University

SUBMITTED IN FULFILLMENT OF THE REQUIREMENTS

FOR THE DEGREE OF

DOCTOR OF PHILOSOPHY

AUGUST 2014 
(C) Copyright by Mir Hojjat Seyedi 2014 All Rights Reserved 
To my wife, Zeinab 


\section{Doctor of PhiLOSOPhy Declaration}

"I, Mir Hojjat Seyedi, declare that the PhD thesis entitled 'A Novel Intrabody Communication Transceiver for Biomedical Applications' is no more that 100,000 words in length including quotes and exclusive of tables, figures, biblography and reference. This thesis contains no material that has been submitted previously, in whole or in part, for the award of any other academic degree or diploma. Except where otherwise indicated, this thesis is my own work."

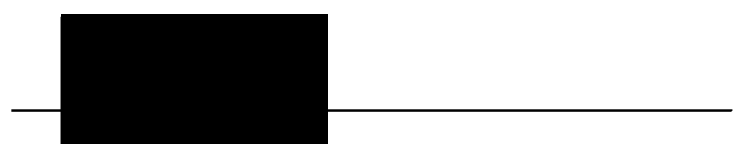

Mir Hojjat Seyedi

Date:June 2, 2014 


\section{Abstract}

Intrabody communication (IBC) is a new physical layer defined in the recently ratified wireless body area network (WBAN) IEEE 802.15.6 standard. The cable-free IBC technology uses body tissue as a propagation medium instead of air. While recent studies have shown a degradation of transmission signal for IBC between limb segments, these degradations have yet to be quantified with respect to relative limb positions. The current thesis investigates the influence of human movement on signal attenuation during IBC considering limb joint effects within the transmission frequency range $0.3-200 \mathrm{MHz}$. In-vivo experiments are conducted to determine the effects of size, situations (flexed or extended), and locations (lower or upper limb) of joints on the IBC. Results show that the presence of joints along the transmission path causes high signal attenuation (up to $6.0 \mathrm{~dB}$ ), the flexed limb exhibits $4.0 \mathrm{~dB}$ less attenuation compared with extended one, and the lower limb joints (knee) shows higher attenuation $(2.0 \mathrm{~dB})$ than upper limb joints, below $60 \mathrm{MHz}$. We propose a new IBC circuit model explaining elbow joint effects. The presented model not only takes the limb joint effects of the body into account but also considers the influence of measurement equipment in higher frequency band thus predicting signal attenuation behavior over wider frequency ranges. Results from the model simulation reveal that the presence of limb joint within the signal transmission path causes an additional 
1.0 to $5.2 \mathrm{~dB}$ loss at frequencies below $60 \mathrm{MHz}$ for on-body channel length of $20 \mathrm{~cm}$. The simulation results suggest that the measurement equipment effects are negligible, below $60 \mathrm{MHz}$. Finally, this work proposes transmitter and receiver architectures for intrabody communication. A carrier-free scheme based on impulse radio (IR) for the IBC (IR-IBC) is implemented on a FPGA. Results demonstrate data rates of up to 1.56 Mbps achievable for the galvanic coupling IBC method. 


\section{Acknowledgements}

In the name of God, the Beneficent the Merciful

During this research and throughout my years in university, I have received insight, support, and encouragement from many people, to whom, I am grateful from the bottom of my heart.

First, and foremost, I would like to extend my sincere gratitude toward my supervisor, Dr Daniel Lai, for his continuous encouragement, academic guidance, and patient support through this entire journey. I would like to thank him for being the source of inspiration and sharing his expert knowledge at every stage of this doctoral study. I am also sincerely grateful to my associate supervisor, Prof. Michael Faulkner, for his practical advices, insightful comments, and critical views through the course of my research. I greatly appreciate his guidance and counseling.

My appreciation also goes to TEPS research students and staff members for creating friendly, supportive, and stimulating atmosphere. I would like to thank Dr. Francois Rivet, Behailu, Zibo, Clement, and Lance for being supportive colleges and coworkers throughout the $\mathrm{PhD}$ study. I am also greatly thankful for the administrative staff of College of Engineering and Science and the Gradate Research Center for their support and assistance in a timely and reliable manner.

Last, but definitely not least, my deepest gratitude goes to my wife for her endless 
love and encouragement and to both our loving families for their tireless support and persistence throughout the whole $\mathrm{PhD}$ journey. 


\section{List of Publications}

\section{Peer-Reviewed Journal Papers}

- M. H. Seyedi, B. Kibret, D. T. H. Lai, and M. Faulkner, "A survey on intrabody communications for body area network applications," IEEE Transactions on Biomedical Engineering, vol. 60, pp. 2067-2079, 2013.

- M. H. Seyedi, Z. Cai, and D. T. H. Lai, "Characterization of signal propagation through limb joints for intrabody communication," International Journal of Biomaterials Research and Engineering (IJBRE), vol. 2, pp. 1-12, 2013.

- M. H. Seyedi and D. T. H. Lai, "The effect of limb joints and limb movement on intrabody communications for body area network applications," Journal of Medical and Biological Engineering (JMBE), vol. 34, pp. 276-283, 2014.

- B. Kibret, M. H. Seyedi, D. T. H. Lai, and M. Faulkner, "Investigation of galvanic coupled intrabody communication using human body circuit model," IEEE Journal of Biomedical and Health Informatics, Accepted, vol. 18, pp. 1196-1206, 2014.

\section{Book Chapter}

- M. H. Seyedi, B. Kibret, S. Salibindla, and D. T. H. Lai, "An overview of intrabody communication transceivers for biomedical applications," Encyclopaedia 
of Information Science and Technology (3rd Ed.), Edited by Mehdi KhosrowPour, IGI Global, Release Date:July 2014.

\section{Refereed Conference Papers}

- M. H. Seyedi, D. T. H. Lai, and M. Faulkner, "Limb joint effects on signal transmission in capacitive coupled intra-body communication systems," in Proc. 34th Annual International Conference of the IEEE EMBS, pp. 6699-6702, CA, September 2012.

- M. H. Seyedi, B. Kibret, D. T. H. Lai, and M. Faulkner, "An empirical comparison of limb joint effects on capacitive and galvanic coupled intra-body communications," IEEE eighth International Conference on Intelligent Sensors, Sensor Networks and Information Processing (ISSNIP), pp. 213-218, Australia, April 2013.

- B. Kibret, M. H. Seyedi, D. T. H. Lai, and M. Faulkner, "An empirical comparison of limb joint effects on capacitive and galvanic coupled intra-body communications," IEEE eighth International Conference on Intelligent Sensors, Sensor Networks and Information Processing (ISSNIP), pp. 318-323, Australia, April 2013.

- A.H.A. Razak, A. Zayegh, R. Begg, M. H. Seyedi, and D. Lai, "BPSK modulation to compare intra-body communication methods for foot planter pressure measurement," rth IEEE GCC Conference, pp. 172-176, Qatar, November 2013.

- Z. Cai, M. H. Seyedi, D. Lai, and F. Rivet, "Characteristics of baseband digital 
signal transmission for intrabody communications," IEEE International Instrumentation and Measurement Technology Conference (I2MTC), pp. 168-190, Uruguay, May 2014. 


\section{Contents}

Abstract $\quad$ v

Acknowledgements vii

List of Publications $\quad$ ix

Contents

List of Tables $\quad$ xvi

List of Figures $\quad$ Xviii

$\begin{array}{ll}\text { List of Symbols } & \text { XXv }\end{array}$

1 Introduction 1

1.1 Human Body Communication . . . . . . . . . . . . . . . . 5

1.2 Objectives . . . . . . . . . . . . . . . . . . 9

1.3 Organization of the Thesis . . . . . . . . . . . . . . . . 11

2 Literature Review 13

2.1 IBC Specifications and Methods . . . . . . . . . . . . . . . . . . . . 14

2.2 Electrical Properties of the Human Tissues . . . . . . . . . . . . . . . 16 
2.3 Modeling Methods of Body Tissues . . . . . . . . . . . . . . . . . . . 19

2.3.1 Human Tissues Parametric Model . . . . . . . . . . . . . . . . 20

2.3.2 Body Channel Circuit Model . . . . . . . . . . . . . . 24

2.3.3 Finite Element Model (FEM) . . . . . . . . . . . . . . 26

2.3.4 Circuit-Coupled FEM Model . . . . . . . . . . . . . 27

2.3.5 FDTD Model . . . . . . . . . . . . . . . . . . . 28

2.3.6 Theoretical Electromagnetic Model . . . . . . . . . . . . . 29

2.4 IBC Transceiver Design . . . . . . . . . . . . . . . . . . . . . . 30

2.5 IBC Challenges . . . . . . . . . . . . . . . . . . . 36

2.6 Summary . . . . . . . . . . . . . . . . . . . 37

3 Experimental Methodology $\quad 39$

3.1 Pre-measurement Preparation and Safety . . . . . . . . . . . . . . . . 40

3.2 Measurement Setup . . . . . . . . . . . . . . . . . . . . 41

3.2.1 On-Body Electrodes . . . . . . . . . . . . . . . . 42

3.2.2 Human Body Channel . . . . . . . . . . . . . . . 44

3.2.3 Electronic Equipment . . . . . . . . . . . . . . . . . 45

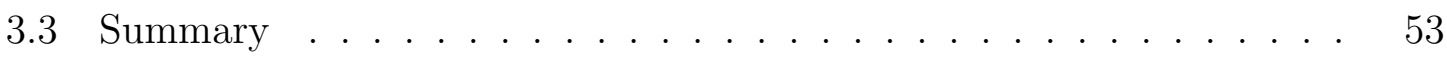

4 Empirical Measurement $\quad 54$

4.1 Influence of Joint-Segments . . . . . . . . . . . . . . . 55

4.1.1 Two-electrode Configuration . . . . . . . . . . . . . . 59

4.1.2 Four-electrode Configuration . . . . . . . . . . . . 62

4.1.3 Discussion ....................... 65

4.2 Effect of Body Posture on IBC . . . . . . . . . . . . . . . . . . 69

4.2 .1 Discussion . . . . . . . . . . . . . . . . . . 74 
4.3 Arm Posture and Effect of Channel Length . . . . . . . . . . . . . . . 75

4.3.1 Discussion . . . . . . . . . . . . . . . . . . . . . 79

4.4 Environmental Effects on IBC . . . . . . . . . . . . . . . . . . 81

4.4.1 Results and Discussion . . . . . . . . . . . . . . 82

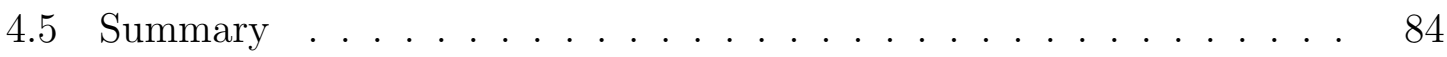

5 Body Channel Modeling $\quad 86$

5.1 Background on IBC Channel Models . . . . . . . . . . . . . . . . 87

5.2 Methods . . . . . . . . . . . . . . . . . . . . 89

5.2.1 Anatomy of the Human Arm . . . . . . . . . . . . . 90

5.2 .2 Limb-Joint Circuit Model _ . . . . . . . . . . . . . . . . . 93

5.3 Measurement and Simulation _. . . . . . . . . . . . . . . . 95

5.3.1 Empirical Measurement . . . . . . . . . . . . . 96

5.3 .2 Circuit Model Simulation _. . . . . . . . . . . . . . 99

5.4 Results . . . . . . . . . . . . . . . . . . 105

5.5 Discussion . . . . . . . . . . . . . . . . . . . . 108

5.6 Summary and Conclusion _. . . . . . . . . . . . . . . 112

6 IBC System Design $\quad 115$

6.1 IBC Hardware Overview . . . . . . . . . . . . . . . . . . . . . . 116

6.2 High Frequency Pulse Transmission . . . . . . . . . . . . . . 120

6.2.1 Pulse Duty Cycle Effect on IBC . . . . . . . . . . . . . . 127

6.3 IBC Hardware Architecture . . . . . . . . . . . . . . . . . . . . 128

6.3 .1 Transmitter Unit . . . . . . . . . . . . . . . . . . 129

6.3.2 Analog Front-End (AFE) for Receiver . . . . . . . . . . . 135

6.3.3 Receiver Unit . . . . . . . . . . . . . . . . . . 137 
6.4 Measurement Results and Discussion . . . . . . . . . . . . . . . . 138

6.5 Summary and Conclusion . . . . . . . . . . . . . . . . . . 143

7 Conclusions and Future Work $\quad 144$

7.1 Challenges of the IBC System Design . . . . . . . . . . . . . 147

7.2 Future Work and Future Directions . . . . . . . . . . . . . . . . 149

$\begin{array}{ll}\text { References } & 151\end{array}$

$\begin{array}{ll}\text { A Appendix } & 166\end{array}$ 


\section{List of Tables}

1.1 Summary of Common RF Protocols Used in WBAN . . . . . . . . . . 4

1.2 Comparison of IBC with NB and UWB Specifications in IEEE 802.15.6 8

2.1 Comparison Between Characteristics of Capacitive and Galvanic Coupling IBC Methods . . . . . . . . . . . . . . . . 17

2.2 Summary and Comparision of Currently Reported IBC Transceivers • 36

4.1 The Signal Attenuation Changes During Joints Motion (Flexion) for Both Configurations of On-Body Electrodes. . . . . . . . . . . . 74

4.2 Variation of Distance between Transmitter ( $\mathrm{Tx}$ ) and Receiver ( $\mathrm{Rx})$ Based on Joints Motion. . . . . . . . . . . . . . . . . . 75

5.1 Thickness of the Tissue Layers Based on Human Arm Radius . . . . . 95

5.2 The Geometries of the Subjects and the Length of the Communication Channel ......................... 101

5.3 The Proposed IBC Models Characteristics Compared to Current Models113

6.1 The Characteristics of IBC Received Signal from the Body of Male

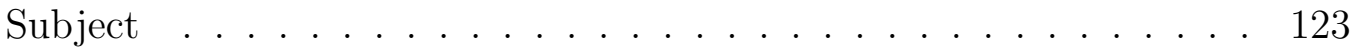

6.2 The Performance Comparision of IBC Systems . . . . . . . . . . . . . 142 
7.1 Summary of Challenges and Future Work in the IBC Technique . . . 150 


\section{List of Figures}

1.1 A communication link is applied between WBAN and WLAN techniques through a healthcare monitoring system. Hub node can act as an information gateway device, transmitting data to hospital access points for analysis or store in electronic record. . . . . . . . . 5

1.2 PHYs of IEEE 802.15 .6 standard. . . . . . . . . . . . . . . . . . 6

2.1 (a) Capacitive coupled IBC. Lines of electric field surrounding the human body are shown in the figure. (b) Galvanic coupled IBC method.

2.2 Current flow establishes between transmitter and receiver electrodes in the galvanic method. The major current travels in short inter-electrode paths in both transmitter and receiver sides. . . . . . . . . . .

2.3 Variation of human tissues electrical properties, relative permittivity and conductivity, against frequency. Three dispersion areas of $\alpha, \beta$, and $\gamma$ are characterized in the figure [48] . . . . . . . . . . . .

2.4 Relative permittivity and Conductivity of human body tissues at different frequencies are plotted based on Gabriel et al. research findings.

2.5 Cole-Cole equation equivalent circuit model for a single time constant. 22

2.6 Current flow in human body tissues [50]. . . . . . . . . . . . . 23 
2.7 Complex (left) and simplified (right) equivalent circuit model of human tissues. The equivalent components of extra-cellular, intra-cellular, and cell membrane are respectively specified by the indexes $e, i$, and $m$ [51]. 24

2.8 Four terminals (electrodes) human arm circuit model during galvanic coupling IBC technique. . . . . . . . . . . . . . . . 25

2.9 Human forearm model is simulated using the circuit-coupled FEM method. The parasitic return path is represented by mean of a capacitor element [57]. . . . . . . . . . . . . . . . . . . . 28

2.10 Simplified block diagram of the IBC transceiver system. . . . . . . . . 31

3.1 Portable residual-current device (RCD) safety switch power outlet. . . 41

3.2 The dedicated measurement setup in IBC method. . . . . . . . . . . . 42

3.3 The employed electrodes in IBC measurement system. . . . . . . . . . 43

3.4 Skin surface electrode designs including metal, gel, and insulator of

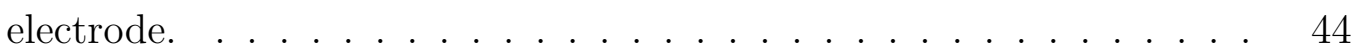

3.5 IBC measurement setup associated with S-parameter. . . . . . . . . . 45

3.6 Measuring the transmission and reflection signal using MiniVNA Pro while the human body acts as DUT. . . . . . . . . . . . . . 46

3.7 The schematic diagram of the employed baluns in the measurement

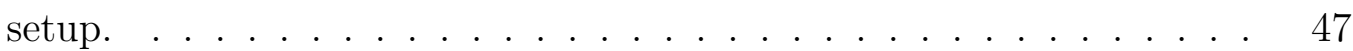

3.8 The balun loss at desired frequency range of this study. . . . . . . . . 48

3.9 The IBC measurement setup: (a) two on-body electrodes and (b) four on-body electrodes configurations . . . . . . . . . . . . . .

3.10 The variation of $S_{21}$ due to the presence of balun in the IBC measurement setup. . . . . . . . . . . . . . . . . 50

3.11 Comparison of signal attenuation through the human body and air. . 51 
3.12 The measured body channel reflection coefficient $\left(S_{11}\right)$ by means of miniVNA Pro. .......................... 52

4.1 Elbow joint anatomy [www.iphysioperth.com.au]. . . . . . . . . 56

4.2 Transmitter and receiver electrodes points over the human body. . . . 58

4.3 Signal attenuation of all subjects during the two-electrode configuration and the presence of elbow joint within the channel. . . . . . . .

4.4 The average (Avr) and standard deviations (SD) of attenuation between three subjects. Results show the effect of elbow joint along the signal pathway during two-electrode IBC configuration. . . . . . . . . 60

4.5 The measured attenuation average (Avr) and standard deviation (SD) between all subjects. The knee joint effect on the IBC signal propagation is demonstrated for two-electrode configuration. . . . . . . . .

4.6 The influence of elbow joint on the signal propagation during fourelectrode configurations. . . . . . . . . . . . . . .

4.7 The influence of elbow joint on signal attenuation during IBC with fourelectrode configurations. The average (Avr) and standard deviation (SD) are calculated between the achieved results from all subjects. . .

4.8 The knee joint effects on signal attenuation during four-electrode configurations. The calculated average (Avr) and standard deviation (SD) are plotted for both channels, with and without joints. . . . . . . .

4.9 Flexion of elbow and knee joints: (a) lifting $10 \mathrm{~cm}$ toes off the floor (b) 90-degree elbow joint angle while the transmitter and electrodes are attached to the body. . . . . . . . . . . . . . . 
4.10 The dedicated measurement setup for joint angle motion experiments. By employing the tape, the position of connecting cables will be fixed on the body. . . . . . . . . . . . . . . . . .

4.11 Elbow joint flexion effects on the IBC signal attenuation for both electrode configurations. Graphs show the average values of attenuations achieved from three subjects. . . . . . . . . . . . .

4.12 Knee joint flexion effects on signal attenuation for both IBC electrode configurations. . . . . . . . . . . . . . .

4.13 The measurement setup; $\theta$ indicates the joint angle between upper and forearm, receiver and transmitter are attached to lower and upper left arm, respectively. . . . . . . . . . . . . . .

4.14 The $90^{\circ}$ flexed arm channel attenuations which measured with the two different cables. . . . . . . . . . . . . . . . .

4.15 Variation of signal attenuation over input signal frequency. Each graph shows the results of 15 and $20 \mathrm{~cm}$ channel length for specified joint angles of $45,90,135$, and 180 degrees. . . . . . . . . .

4.16 The value of average attenuations at different joint angles for two frequencies of (a) $2.0 \mathrm{MHz}$ and (b) $60 \mathrm{MHz}$ for channel lengths of $15 \mathrm{~cm}$

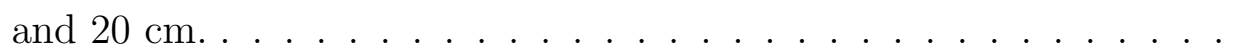

4.17 The average (Avr.) and standard deviation (SD) of the IBC channel loss in three different environments for two-electrode configurations. .

4.18 The average (Avr.) and standard deviation (SD) of the IBC channel loss in three different environments for four-electrode configurations. .

5.1 Current propagation through body and air, current path 1 and 2 respectively, during four-electrode IBC configuration . . . . . . . . . . 
5.2 Geometrical human arm model including four on-body electrodes and presence of the elbow joint in the middle of signal transmission path between transmitter (TX) and receiver (RX) electrodes. (a) Human body communication channel is involved the limb joint (b) No joint is

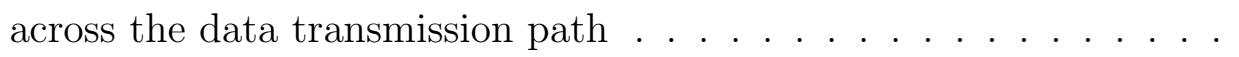

5.3 Proposed circuit model of human arm considering elbow joint during four-electrode IBC configuration: Circuit model A. . . . . . . . . .

5.4 The body channel circuit model including measurement equipment model: Circuit model B.

5.5 The measurement setup of IBC using four on-body electrodes during no joint (left) and with joint (right) positions. . . . . . . . . . . . 96

5.6 The results of empirical measurements on a body arm using IBC. . . 98

5.7 (a) Circuit model A: simulation of the human arm in presence of the elbow joint. (b) Circuit model B: simulation by adding the cables and Baluns model. . . . . . . . . . . . . . . . . . . . . . 100

5.8 The transverse current flow between two electrodes of transmitter or receiver through the inner layers of human tissue. . . . . . . . . . . . 102

5.9 The empirical measurement results to determine the electrode-skin impedance. $\mathrm{Z}$ is the combination of electrode-skin and input impedance of the body $\left(Z_{i}\right) \ldots \ldots \ldots \ldots \ldots$

5.10 The measurement results of electrode-skin impedance. . . . . . . . . . 104

5.11 The comparison of the air path and the body path impedances between the transmitter and receiver electrodes. . . . . . . . . . . . . 105

5.12 Measurement and model simulation (without cables and baluns model) results on human arm as a transmission path. . . . . . . . . . 106 
5.13 The comparison of the empirical and model B simulation results. The effects of cables and baluns were considered in the simulation. . . . . 107

5.14 The RMSE between empirical and simulation for both proposed models.108

5.15 Signal attenuation at different elbow joint length $\left(L_{j}\right) . \quad \ldots . .109$

6.1 Bidirectional IBC Transceiver. . . . . . . . . . . . . . . . . . . 117

6.2 The measurement setup of the IBC technique. . . . . . . . . . . . . 121

6.3 Transmitted pulse wave $(50 \mathrm{MHz})$ through the body using IBC method.122

6.4 The signal attenuation of the arm for 0.3-200 MHz. . . . . . . . . . . 124

6.5 IBC received signal in $50 \mathrm{MHz}$ for both female male test subject. . . 125

6.6 IBC received signal in $150 \mathrm{MHz}$ for both female male test subject. . . 126

6.7 The signal attenuation of the body channel for input signal with 20\%$50 \%$ duty cycle when Data Generator was used as transmitter. . . . . 127

6.8 The architecture of proposed IBC PPM. . . . . . . . . . . . . . . 130

6.9 A simplified architecture of the IBC PPM transmitter. . . . . . . . . 131

6.10 A generated PPM signal, $s(t)$, and the output of pulse generator, $p(t)$, in the IBC PPM architecture. . . . . . . . . . . . . . . . 132

6.11 The output of IBC transmitter, in+ and in-, which transmit through the body. . . . . . . . . . . . . . . . . . 133

6.12 The transmitter output pattern in the proposed IBC system. . . . . . 134

6.13 The schematic of receiver AFE used in the proposed IBC system. . . 135

6.14 The output signal of AFE compared to the generated signal by transmitter, $s(t)$ during the body channel length of $14 \mathrm{~cm}$. The phase-offset and noise is shown in the figure. . . . . . . . . . . . . 136

6.15 The receiver $(\mathrm{RX})$ unit of the proposed IBC system. . . . . . . . . . . 137

6.16 Spectrum measured at PPM transmitter output. . . . . . . . . . . 138 
6.17 BER calculation through the measurement results of PPM IBC system. The error bits are indicated in red color. . . . . . . . . . . . . . . . 139

6.18 The measurement setup of galvanic coupling IBC using FPGA board for calculating the system BER. . . . . . . . . . . . . . . . . 140

6.19 BER Performance of PPM IBC system. . . . . . . . . . . . . . . . . 141

A.1 The top-level register-transfer level (RTL) schematic of the proposed IBC tranceiver in this syudy. . . . . . . . . . . . . . . 166 


\section{List of Symbols}

\begin{tabular}{|c|c|}
\hline$f_{B W}$ & bandwidth frequency............ \\
\hline$\epsilon_{r}$ & relative permittivity..... \\
\hline$\sigma$ & electrical conductivity...... \\
\hline$\tau$ & relaxation time constant \\
\hline$\alpha_{n}$ & distribution parameter \\
\hline$\sigma_{i}$ & 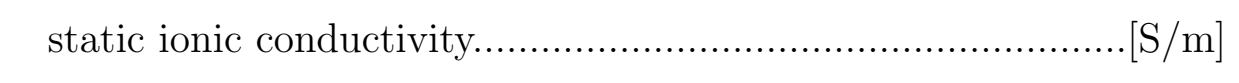 \\
\hline$Z^{*}$ & complex specific impedance................... \\
\hline$Z_{C P E}^{*}$ & constant phase element impedance .... \\
\hline$L_{A}$ & arm length...... \\
\hline$D_{A}$ & arm diameter. \\
\hline$L_{j}$ & joint length..... \\
\hline$L_{F A}$ & forearm length... \\
\hline$L_{U A}$ & upper arm length.. \\
\hline$A$ & tissue cross section area.. \\
\hline$d$ & longitudinal distance between electrodes.... \\
\hline$Z_{i}$ & body transversal impedance (input) \\
\hline$Z_{o}$ & body transversal impedance (output) ......... \\
\hline$b$ & 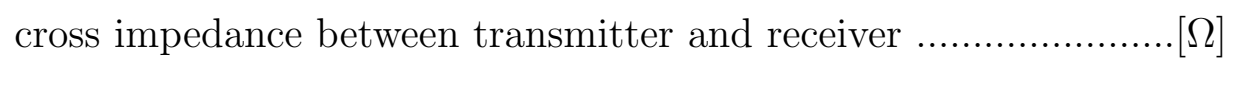 \\
\hline
\end{tabular}




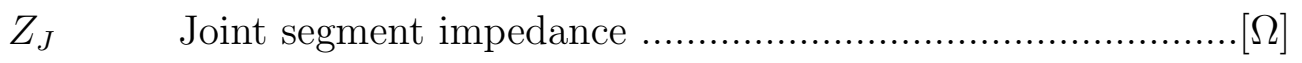

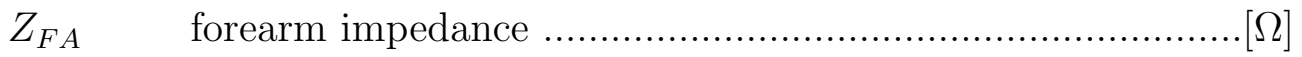

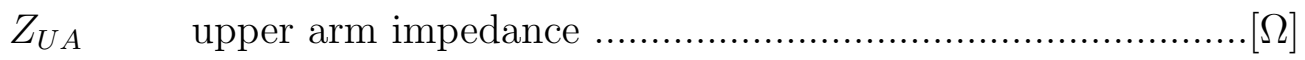

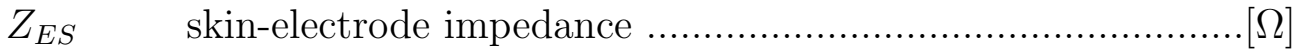




\section{Chapter 1}

\section{Introduction}

Imagine a world in which as you go about your daily life, your health is continuously monitored. Such systematic monitoring helps to reduce the reliance on medical personnel for current medical disorders like heart attacks or stroke [2]. It could minimize hospital visits, cut costs for both patient and healthcare system, and improve the quality of healthcare monitoring. According to recent research from New England Healthcare Institute (NEHI, 2009), up to $\$ 6.4$ billion could be saved annually by only U.S. patients at risk of heart failure through minimising the excessive need for hospital visits and the associated medical costs [3]. Based on a report by the Australian Academy of Technological Sciences and Engineering (ATSE) in 2010, the potential cost saving of patients' healthcare monitoring from home is estimated up to $\$ 520$ million per year for the Government [4]. The Federal Government of Australia has included remote patient monitoring as one of its main cost saving solutions in the healthcare system. Apart from medical applications, real-time healthcare monitoring is in high demand for sport where a personalized training plan for each athlete should be developed by coaches to improve the performance of their team, especially at an 
elite level. To this end, the physiological capabilities, physical activities, and vital signs of athletes need to be closely monitored and recorded by the coaches through long-term monitoring [5]. According to a report by ABI Research, the market for sports and fitness-related monitoring devices is projected to reach 80 million device sales by 2016 [6].

Nowadays, portable monitoring devices have herald a revolutionary change in healthcare monitoring systems. They are capable of logging and monitoring physiological data and recording basic vital signs, routinely. For instance, Myo Trac Infiniti is a recent handheld portable electromyography (EMG) system introduced by Thought Technology Ltd. (NY, USA) in 2003. It has a smaller size $(102 \mathrm{~mm} \times 152 \mathrm{~mm} \times 51 \mathrm{~mm})$ and lighter weight (330 g with batteries) compared to conventional PC-based EMG monitoring systems, such as Nicolet Viking $I V$, which makes it suitable for real-time medical measurements. The high quality of monitoring as well as the increased freedom and mobility of the patients or athletes distinguish the portable monitoring methods from traditional wired techniques.

The current paradigm in healthcare is the notion of continuous remote patient monitoring using electronic devices with sensors. The sensors are miniature and lightweight, commonly worn or implanted in the body. For instance, the eMotion electrocardiogram (ECG) sensor (Mega Electronics Ltd., Finland) could continuously transmit the patient's heart rate to a mobile phone via Bluetooth. Further, an implantable blood glucose sensor from Pepex Biomedical, Inc. (AZ, USA) is capable for continuous monitoring of the blood sugar levels. The sensors avoid the need for a manual self-administered health system and may enable users to take control of their health disorders in the future.

Currently, multiple sensors attached to different body locations for monitoring 
patients' vital signs are widely used in health care monitoring due to their high recognition accuracy and low computational load [7]. For instance, brain and muscles activities need to be continuously monitored in patients with Parkinson's disease [8]. The 192-channel simultaneous recording, i.e. large number of electroencephalography (EEG) sensors, are employed to get more data from a larger area of the head. Therefore, creating a network of sensors and providing new wireless standards, such as wireless body area network (WBAN), instead of cabling all sensors to the processing unit will improve portability. Employing more sensors provides higher data resolution, yet increases the bandwidth requirement of the network and power consumption.

Wireless communication technologies provide new possibilities for clinicians to remotely sample biomedical data [10]. Currently wireless medical telemetry systems (WMTS) are employed for patient monitoring using well-known wireless protocols such as WLAN (wireless local area network) [11], and short range communication by means of IEEE 802.15.1 (Bluetooth) and IEEE 802.15.4 (ZigBee). In a recent WBAN for live sport monitoring, 40000 runners of the Paris Marathon were equipped with 120000 (3 sensor nodes per athlete) on-body sensors through IEEE 802.15.4 for data communication [12]. Generally, these wireless protocols operate at radio frequency (RF) bands for data transmission within long or short distances. Table 1.1 shows the characteristics of some protocols which are currently developed in biomedical applications.

A major drawback of wireless RF propagation for miniaturized medical systems, especially portable monitoring devices, is the high power consumption which limits the practical duration of operation. Most current studies claim that ZigBee and ANT have a battery life of three years, but this is at a low operating data rate, for example 1 byte transmitted every 5 minutes [13]. Further, the IEEE 802.15.4 standard for 
Table 1.1: Summary of Common RF Protocols Used in WBAN

\begin{tabular}{|c|c|c|c|}
\hline Porotocol & $\begin{array}{c}\text { Frequency } \\
{[\mathrm{GHz}]}\end{array}$ & $\begin{array}{c}\text { Data rate } \\
{[\mathrm{bps}]}\end{array}$ & $\begin{array}{c}\text { Coverage area } \\
{[\mathrm{m}]}\end{array}$ \\
\hline \hline $\begin{array}{c}\text { WLAN } \\
\text { (IEEE } \\
802.11 \mathrm{a} / \mathrm{g} / \mathrm{n})\end{array}$ & $2.4-5.1$ & $54 \mathrm{M}$ & $35-250$ \\
\hline $\begin{array}{c}\text { Bluetooth } \\
(\mathrm{IEEE} \mathrm{802.15.1)}\end{array}$ & 2.4 & $0.1-24 \mathrm{M}$ & $1-10$ \\
\hline $\begin{array}{c}\text { ZigBee } \\
(\mathrm{IEEE}\end{array}$ & 2.4 & $250 \mathrm{k}$ & $10-75$ \\
\hline 802.15 .4$)$ & 2.4 & $1 \mathrm{M}$ & 30 \\
\hline ANT & & & \\
\hline
\end{tabular}

low power Zigbee protocol specifies a transmission power output of $0 \mathrm{dBm}(1 \mathrm{~mW})$. Continuous operation at the maximum data rate of $250 \mathrm{kbps}$ generally consumes a normal Lithium ion battery in a matter of hours. On the other hand, Bluetooth (classic) provides at least 1.0 Mbps while approximately consuming $148.5 \mathrm{~mW}$ during on-body communication [14]. It is evident that new approaches to ultra-low power wireless technology are required to improve next generation wireless technologies for medical body area network (BAN) applications. According to Hanson et al. [15], a peak power consumption of WBAN in the active mode should be at the most $30 \mathrm{~mW}$ and $0.001-0.1 \mathrm{~mW}$ in stand-by mode.

Figure 1.1 shows data transmission among multiple sensors using WBAN technologies within and around the body and a communication link between decision maker (usually hub node) and wireless LANs technologies. For instance, in the Paris Marathon WBAN, the hub node was located on a motorbike a few meters ahead of the runner during the whole race [12]. 


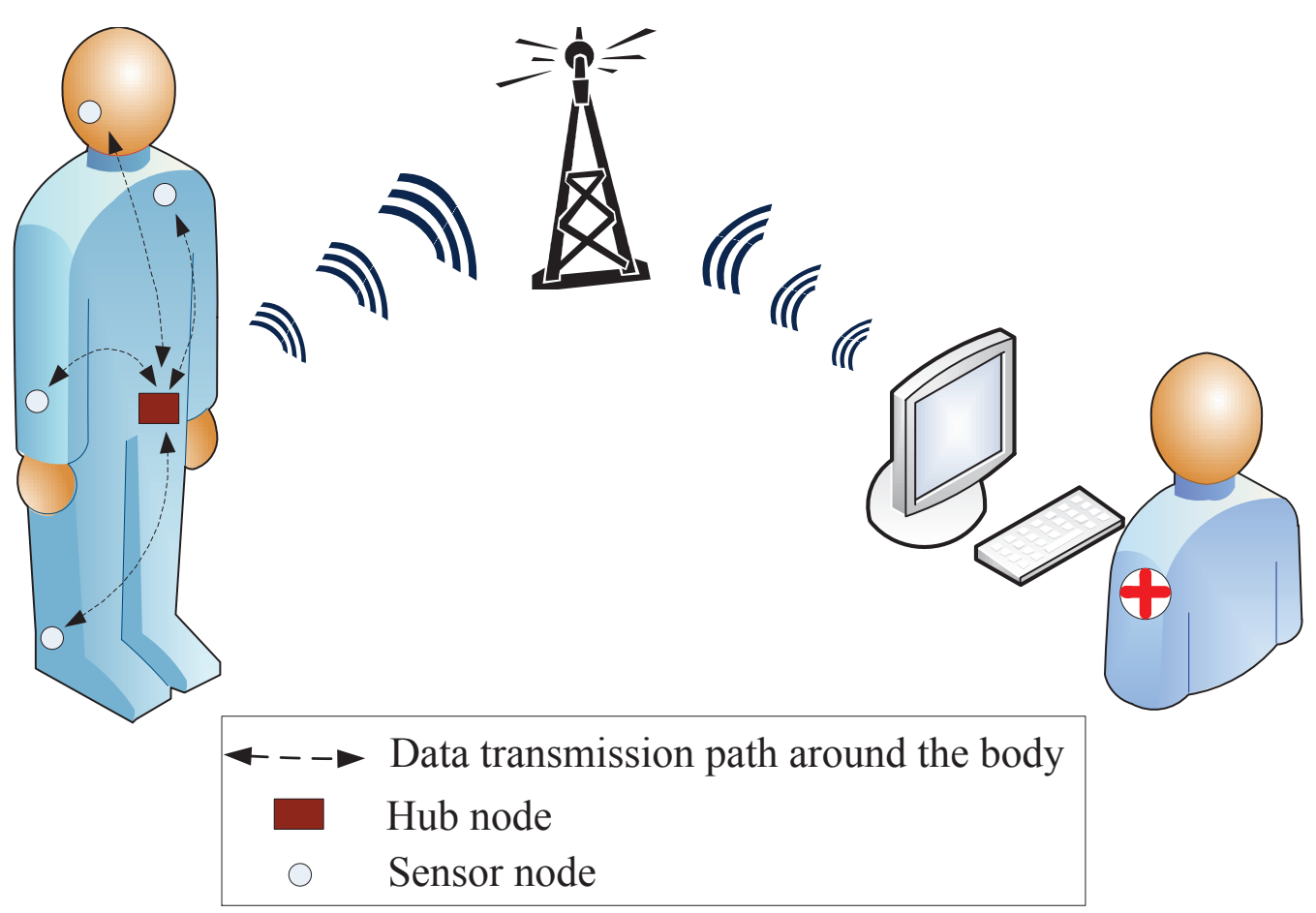

Figure 1.1: A communication link is applied between WBAN and WLAN techniques through a healthcare monitoring system. Hub node can act as an information gateway device, transmitting data to hospital access points for analysis or store in electronic record.

\subsection{Human Body Communication}

In the early 2012, the standardization of a new WBAN protocol, IEEE 802.15.6 was ratified by task group TG6 that defines the new proprietary physical layers (PHYs) for sensor networks around the body: Narrowband (NB), Ultra-wideband (UWB), and Human body communication (HBC) PHY. The operation of the first two is based on $\mathrm{RF}$ propagation, while the latter is a new non-RF communication technique based on data transmission through human body tissues [16].

Multiple frequency bands of operation supported by NB PHY include the 402$405 \mathrm{MHz}$ for implantable devices, three different frequency bands (863-956 MHz) for wearable applications, and finally $2360-2400 \mathrm{MHz}$ for medical demands. UWB 


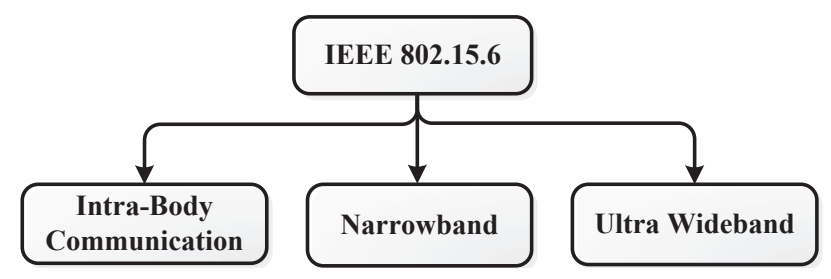

Figure 1.2: PHYs of IEEE 802.15.6 standard.

PHY operates in the highest frequency regions, particularly the $3-5 \mathrm{GHz}$ and the 6-10 GHz bands with channel frequency bandwidth $\left(f_{B W}\right)$ of $499.2 \mathrm{MHz}$ [17]. Data rates of NB PHY range between 100 and 1000 kbps and UWB PHY range from 395 kbps to 12.636 Mbps in the mandatory mode. NB and UWB suffer from high signal attenuation through the human body (more than $60 \mathrm{~dB}$ loss [18]) which will increase the power consumption of WBAN devices. Since the WBANs devices are designed to operate under both dynamic and static conditions of their users, the body shadowing effects can be a critical issue while employing NB and UWB technologies in WBAN implementations [19]. In addition, these devices are often operated in the licensefree industrial, scientific and medical (ISM) radio band which is an over crowded radio spectrum (shared by any device that uses Bluetooth, Zigbee and ANT) which would affect the reliability of the communicated data and the network coexistence, particularly when many users are in close vicinity.

HBC is a novel data transmission technique using the human body itself as the transmission medium or channel. This method is set to eliminate both bulky cable and wireless antenna from medical monitoring communication devices. Compared to RF wireless techniques, HBC potentially provides lower transmission power (less than 1.0 $\mathrm{mW}$ ) with data rates of more than $100 \mathrm{kbps}$ [20]. According to WBAN standard [17] the operating frequency band of $\mathrm{HBC}$ is centered at $21 \mathrm{MHz}$ with $f_{B W}=5.25 \mathrm{MHz}$ and scalable data rates of 164-1312.5 kbps. HBC has been referred by other authors 
as body channel communications (BCC) [21] or intrabody communications (IBC). In this thesis, we will use IBC to denote this communication method as the study will cover work outside the IEEE 802.15.6 standard. The IBC technique has been touted to have the following desirable characteristics:

- Security: The IBC system is a protected and private communication network which provides natural security and low interference communication [22]. The required operating frequency of IBC is much lower compared to RF systems. This means signals are confined to the person's proximity since reading data requires body contact to be made [23]. At higher frequencies (100 $\mathrm{MHz}$ to several gigahertz), the signal wavelength becomes comparable to the human body channel length and the body radiates energy, acting as an antenna (dipole antenna) [24]. Since transmitter and receiver contain small size electrodes (for example Neuroline electrodes active area is $54 \mathrm{~mm}^{2}$ ) instead of antennas, the larger wavelength of the carrier signal compared to the electrode size results in low interference IBC.

- Energy efficiency: The key issue with RF propagation in portable devices is that it consumes battery life quickly. For example, ZigBee has maximum data rate of $250 \mathrm{kbps}$ at $26.5 \mathrm{~mW}$ resulting in $106 \mathrm{~nJ}$ per received bit [21]. The energy consumption of UWB is $2.5 \mathrm{~nJ} / \mathrm{b}$ when the data rate is $16.7 \mathrm{Mbps}$. Recent research results demonstrated that IBC consumes an order of magnitude less energy $(0.24 \mathrm{~nJ} / \mathrm{b})$ at data rates up to $10 \mathrm{Mbps}$ which makes it an attractive communications method for WBAN applications [21].

- Frequency reuse: IBC forms a short range communication network inside and around human body and therefore allows the same frequency band to be reused 
Table 1.2: Comparison of IBC with NB and UWB Specifications in IEEE 802.15.6

\begin{tabular}{|c|c|c|}
\hline & IBC & $\mathrm{NB}, \mathrm{UWB}$ \\
\hline Communication medium & Human body & Air \\
\hline Frequency band & $\begin{array}{c}\text { Centered at } 21 \mathrm{MHz} \\
\left(f_{B W}=5.25 \mathrm{MHz}\right)\end{array}$ & $\begin{array}{l}\text { Different bands } \\
\text { (402 M-10 GHz) }\end{array}$ \\
\hline Data rate & less than 2 Mbps & less than $13 \mathrm{Mbps}$ \\
\hline Transmission Range & less than $2 \mathrm{~m}$ & $10 \mathrm{~m}$ \\
\hline Signal Attenuation & Low & $\begin{array}{c}\text { High } \\
\text { (Body Shadowing) }\end{array}$ \\
\hline On-Body Antenna requirement & No & Yes \\
\hline Energy Efficiency High & $\begin{array}{c}\text { High } \\
\text { (High Conductivity } \\
\text { of the human body) }\end{array}$ & $\begin{array}{c}\text { Low } \\
\text { (Air has low } \\
\text { conductivity) }\end{array}$ \\
\hline
\end{tabular}

by WBANs on other users (coexistence with other WBANs) with minimal interference. This property potentially allows future designs to focus on improving data rates, reducing power consumption, and integrating the transceivers into smaller form factors [31]. Table 1.2 adapted from [17], [32-34], situates the IBC technique with respect to RF WBAN and provides a brief comparison between their specifications.

Although the advantages of IBC have been reflected by several researchers in terms of reliability and energy efficiency, the IBC related attempts are still in their infancy. Several studies have been generally conducted to tackle some major issues of the IBC system design such as communication data rate [25], communication noise and interference, the electromagnetic (EM) radiation $[22,26]$, and so on. Further, more recent studies have concisely indicated that different body positions affect data transmission during IBC [27]. These studies have investigated the variation of signal 
communication through the body during complex body movement, such as walking on treadmill [28]. Yet, the concern for developing an efficient IBC system extends beyond the investigation of the complex body movement. As Schaal [29] stated, complex body motions consist of smaller segments called "units of action" or "movement primitives". While previous studies have shown degradations of transmission signal for the complex movement sequences, these degradations have yet to be quantified with respect to relative "units of action". According to [30], whole body movements are mainly permitted by joint limbs. Therefore, the precise study of limb joints effects on signal communication needs to be considered through the "units of action". While body motion effects on IBC are subject to change in response to various internal (e.g. body tissue composition) and external factors (e.g. surrounding environment), quantification of the transmission signal loss in the presence of limb joints will improve understanding of the IBC method's limits. According to Wegmueller et al. [9], the transmission loss caused by the body limbs with massive amount of bone, such as limb joints, is higher compared to the limbs with less bone. Since research studies on IBC have rarely explored the variations of signal attenuations in the 'unit of action' scales, this study aims to deal with this issue by focusing on different joint sizes, joint situations (extended or flexed), and joint locations (upper or lower limb).

\subsection{Objectives}

The characteristics of IBC such as the low operation frequency, low interference wireless communication, and natural security (i.e. physical contact needs to be made to read the transmitted data) make it enticing for short-range communication scenarios. Since the human body is employed as a communication channel in IBC technique, the 
body postures in different situations have inevitable effects on IBC systems design. This raises a further concern about the influence of body motion on the data transmission through the human body. Currently, not a lot is known about the influence of body smaller segments motion, i.e. 'units of action', on the IBC system. Hence, the major aim of this research is to investigate the effects of body movements on IBC. For this purpose, we investigate the effects of limb joints, as a limb causes whole body movements, whereas they place across the data transmission path. As a first step, comprehensive empirical measurements will be carried out on the real human body. The experimental results will enable us to derive and verify a suitable human body model. Therefore, in second step, to understand the body channel characteristics and constraints, simulation models will be considered. Finally, the effect of joint between the proposed on-body transmitter and receiver during square wave communication will be examined. The results will be used to develop new energy efficient IBC system. Therefore our study specifically aims to:

1. investigate the presence of limb joints on the signal transmission path during different methods of IBC.

2. investigate the operation of IBC on lower and upper limbs and influence of external ground on the signal communication.

3. develop a new model of the human body as a communication channel while considering the body's limb joint effects.

4. develop a new IBC transmitter and receiver design based on the experimental results and modeling. 


\subsection{Organization of the Thesis}

This thesis consists of 6 parts and each part is compiled as a chapter. A brief outline of work in each chapter is presented.

- Chapter 2 introduces in details both IBC coupling methods, capacitive coupling and galvanic coupling. It reviews the technical aspects of IBC such as communication channel modeling and transceiver designs presented by recent research. The remaining challenges in the proposed IBC systems are also surveyed in this chapter.

- Chapter 3 presents an explanation of the research methodology as well as experimental equipment. In addition, IBC testing safety requirement and measurement setups for both IBC methods used in later chapters are discussed and detailed.

- Chapter 4 highlights new empirical results which demonstrate that body motion (in term of joint movement) affects signal attenuation during IBC methods. In this chapter, the experimental details and results for in-vivo measurements are examined. Results contain the investigation of IBC method with two and four electrodes attached to the body. The experiments are carried out on upper and lower limbs of the human body. The obtained results are analyzed and discussed for each measurement.

- Chapter 5 proposes a new circuit model for IBC considering limb joint and measurement equipment. The achieved results from the model are compared with the real body measurements results in chapter 4 . The characteristics of our model are analyzed and compared with previous IBC models. The results 
demonstrate that the signal propagates through the body tissues below $54 \mathrm{MHz}$ where the effects of measurement equipment such as cables are negligible.

- Chapter 6 presents the behavior of square waves while transmitting through the body. The effects of the elbow joint, signal duty cycle, and the transmission frequency are examined in this chapter. Finally, a digital baseband IBC system based on impulse radio (IR) is designed and implemented in FPGA. We have developed a receiver analog front-end (AFE) using off-the-shelf components for an energy efficient IBC system design. The results demonstrate that the proposed IBC architecture could provide a data throughput of $1.56 \mathrm{Mbps}$ with the low power consumption of $3.94 \mathrm{~mW}$.

- Chapter 7 highlights the remaining challenges and the required future research based on some of our own experimental results and the latest IEEE 802.15.6 standard. 


\section{Chapter 2}

\section{Literature Review}

The term "Body Area Network" (BAN) was first introduced by Van Dam et al. [36] in 2001. BAN technology envisions miniaturized sensors worn [37] or implanted on the body, continuously monitoring health parameters and acting to prevent the onset of critical health events. For instance, diabetics currently have access to an automatic insulin pump which monitors glucose levels and administers insulin when glucose levels are high. Similar technologies are also leading toward the construction of devices which can minimize incidences of heart attack or stroke, thus reducing hospital visits and saving costs for both the individual patient and a nation's healthcare system. According to a recent report from Parks Associates, the U.S. market for wireless home-based healthcare applications and services are expanding with an annual growth rate of over $180 \%$ and becoming a $\$ 4.4$ billion industry in 2013 [38]. Such statistics indicate a rising demand for portable health monitoring devices, e.g. BANs, which are currently undergoing tremendous research and development.

This chapter aims to compile the latest research on a new form of wireless communications in BAN which is fast gaining attention. IBC is a novel non-RF wireless 
data communication technique which uses the human body itself as transmission medium for electrical signals. The inhibition of communication to the person's proximity in IBC users prevents the energy from being dissipated into the surrounding environment, resulting in potentially lower power consumption. Research has shown that IBC is capable of low transmission power below $1 \mathrm{~mW}$ and data rates of more than $100 \mathrm{kbps}$ [20] which makes this approach potentially appealing as a short range communications alternative.

This chapter is organized as follows: Section 2.1 briefly introduces the IBC technique which is primarily based on capacitive or galvanic coupling. Section 2.2 presents the effects of human body tissues dielectric properties on signal transmission through the body. The various human body communication channel modeling methods and their characteristics are reviewed in section 2.3. The final section discusses the latest state of the art transceiver designs and implements.

\subsection{IBC Specifications and Methods}

In general, IBC can be classified into two basic coupling types (i.e. how the electrical signals are transmitted): capacitive coupling (near-electric field) and the galvanic coupling (Waveguide). Figure 2.1 illustrates schematically the two different types of IBC coupling. For both coupling types both transmitter and receiver needs two pair of electrodes each. In capacitive coupling only one of the electrodes, i.e. signal electrode, of the transmitter side and receiver side is attached to the body while the other electrode (ground electrode) is floating. In the galvanic coupling method both electrodes of transmitter and receiver side are attached to the human body. The theory of capacitive coupled IBC is established based on the capacitive coupling of 


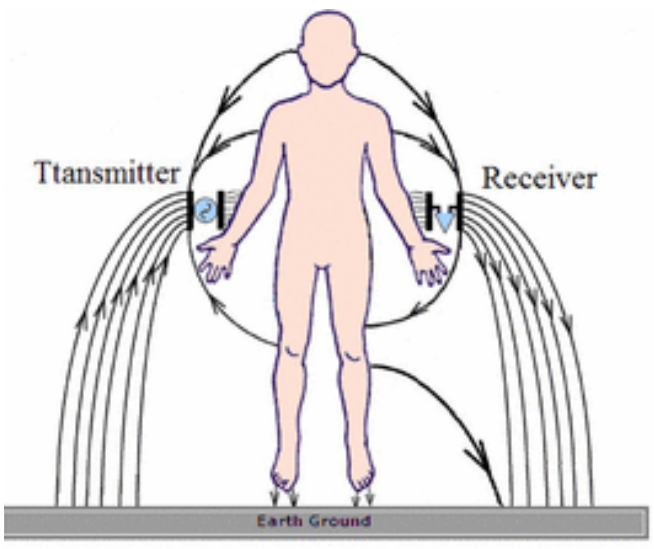

(a)

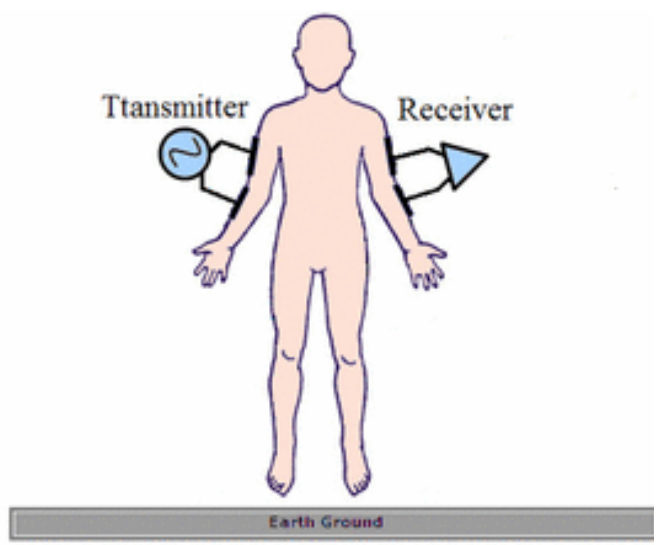

(b)

Figure 2.1: (a) Capacitive coupled IBC. Lines of electric field surrounding the human body are shown in the figure. (b) Galvanic coupled IBC method.

the human body to its surrounding environment. The signal is generated between the body channel transceiver by making a current loop through the external ground. The signal electrode of the transmitter induces the electric field in to the human body. The induced electrical signal is controlled by an electrical potential and the body acts as a conductor with the ground as the return path. Data propagation by capacitive coupling through the human body was first proposed by Zimmerman as means of communications in a personal area network (PAN) [39].

On the other hand, galvanic coupling is achieved by coupling alternating current into the human body. It is controlled by an AC current flow and the body is considered as a transmission line (waveguide). In the galvanic coupled IBC an electrical signal is applied differentially between the two electrodes of the transmitter. Major propagation of the signal occurs between the two transmitter electrodes and a largely attenuated signal is received by the two receiver electrodes. Figure 2.2 shows pathways for a current flow between transmitter and receiver electrodes in the galvanic method. The small current also results in a differential signal between the electrodes 


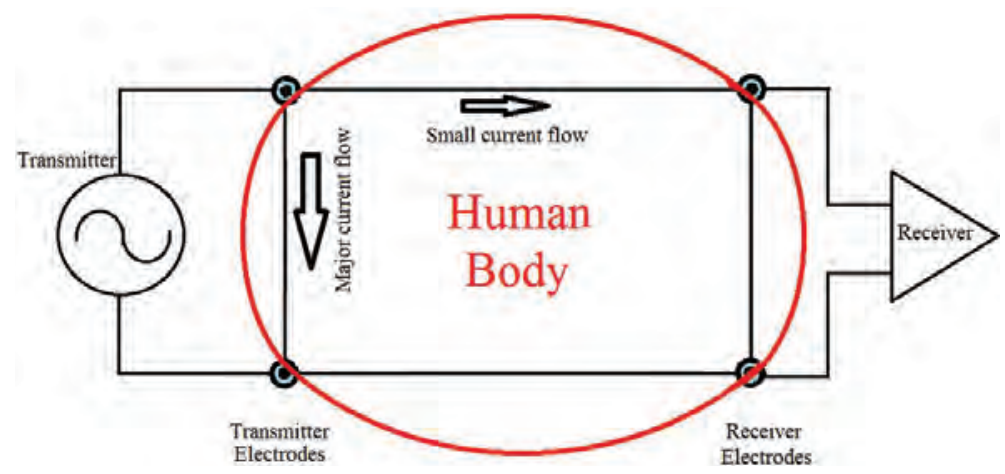

Figure 2.2: Current flow establishes between transmitter and receiver electrodes in the galvanic method. The major current travels in short inter-electrode paths in both transmitter and receiver sides.

of the receiver. In general, the ion content in the human body is the carrier of information in the galvanic coupling method. The principles of galvanic coupling IBC was first introduced by Oberle [40] while designing a single chip low power biomedical system. Table 2.1 briefly compares capacitive and galvanic coupling methods.

\subsection{Electrical Properties of the Human Tissues}

Propagation of galvanically or capacitively coupled signals through the human body in IBC is largely governed by human tissue electrical properties. The two major properties are relative permittivity $\left(\epsilon_{r}\right)$ and electrical conductivity $(\sigma)$. The relative permittivity and electrical conductivity of a material are, respectively, the dipole and current densities induced in response to an applied electric field of unit amplitude [44]. Tissue types, the operation frequency range, temperature, intactness of cellular membranes, and tissue water content are some of the major factors which determine the tissue electrical properties in the human body.

The most comprehensive overview on human body electrical properties is presented by Gabriel et al. in 1996 [1]. Experiments were performed on human and 
Table 2.1: Comparison Between Characteristics of Capacitive and Galvanic Coupling IBC Methods

\begin{tabular}{|c|c|}
\hline Capacitive Coupling (Electric Field) & Galvanic Coupling (Waveguide) \\
\hline $\begin{array}{l}\text { The induced signal is controlled by } \\
\text { an electrical potential (Applying static } \\
\text { charged electrode). }\end{array}$ & $\begin{array}{l}\text { The induced signal is controlled by a } \\
\text { current flow (alternating currents over } \\
\text { multiple electrodes). }\end{array}$ \\
\hline $\begin{array}{l}\text { Only signal electrodes of the transmit- } \\
\text { ter and the receiver are attached to } \\
\text { the body, while both ground electrodes } \\
\text { float. }\end{array}$ & $\begin{array}{l}\text { A pair of transmitter and receiver elec- } \\
\text { trodes is attached to the body. }\end{array}$ \\
\hline Ground is required as a reference. & Ground is not required as a reference. \\
\hline $\begin{array}{l}\text { The dominant signal transmission } \\
\text { pathway is the environment [31]. }\end{array}$ & $\begin{array}{l}\text { The dominant signal transmission } \\
\text { pathway is the body tissue. }\end{array}$ \\
\hline $\begin{array}{l}\text { Higher transmission date rate and } \\
\text { channel gain (Higher operation fre- } \\
\text { quency compared to galvanic coupling) } \\
{[21] \text {. }}\end{array}$ & Lower transmission date rate [9]. \\
\hline $\begin{array}{l}\text { The human body is modeled as a vol- } \\
\text { ume conductor (body is approximated } \\
\text { as a single node }[41] \text { ). }\end{array}$ & $\begin{array}{l}\text { The body is modeled as a waveguide for } \\
\text { signal conduction [42]. }\end{array}$ \\
\hline $\begin{array}{l}\text { Signal quality influenced by the envi- } \\
\text { ronment around the body. }\end{array}$ & $\begin{array}{l}\text { Signal quality influenced by dielectric } \\
\text { properties of human tissue. }\end{array}$ \\
\hline $\begin{array}{l}\text { Interference from surrounding devices } \\
\text { that could capacitively couple directly } \\
\text { to the IBC device. }\end{array}$ & $\begin{array}{l}\text { Sensitive to the body location because } \\
\text { of the dependence on inter-electrode } \\
\text { distance and orientation along the } \\
\text { body. }\end{array}$ \\
\hline $\begin{array}{l}\text { Does not require direct contact with } \\
\text { the human body. Needs only to be in } \\
\text { the proximity. }\end{array}$ & $\begin{array}{l}\text { Direct contact with body tissue is nec- } \\
\text { essary. Capable of both on-body and } \\
\text { in-body (implanted) device communi- } \\
\text { cation [43]. }\end{array}$ \\
\hline
\end{tabular}

animal tissue within the frequency range of $10 \mathrm{~Hz}$ to $20 \mathrm{GHz}$. During the experiments the temperature was fixed $\left(37^{\circ} \mathrm{C}\right)$ and it was assumed that tissue layers were 
homogenous. The electrical properties of a living tissue were measured through the interaction between electromagnetic radiation and tissue cells. Additional research revealed that dielectric properties of living tissue vary differently with frequency dispersion. Frequency dispersion mechanism was first introduced by Schwan [45] to characterize the electrical properties of biomaterials. The dispersion refers to the behavior of tissues at various frequency ranges; low frequency ranges, RF ranges, and gigahertz frequency ranges which are respectively referred to as Alpha, Beta, and Gamma dispersion. Figure 2.3 shows the dispersion of relative permittivity and specific conductivity of human body tissue. The major characteristics of biological tissues can be summarized as follows:

- Permittivity strongly declines, whereas conductivity increases within these frequency dispersions.

- The polarization of water molecules creates the gamma $(\gamma)$ distribution in the gigahertz region (microwave frequencies). The gamma dispersion is not strong and it has minimal effect on the electrical properties of body tissues which carry protein bound water.

- The polarization of cellular membranes is an obstacle for an ion to flow in, or out of the cell and leads to the beta $(\beta)$ dispersion. This region lies within hundreds of kilohertz to ten megahertz range. The polarization of protein is another contributing factor to the beta dispersion trend.

- The transport of ions across a biological membrane is related to the low frequency alpha $(\alpha)$ dispersion. The alpha dispersion can be found in frequency range between $1 \mathrm{~Hz}$ up to $100 \mathrm{kHz}$. An increase in tissue conductivity is rarely 


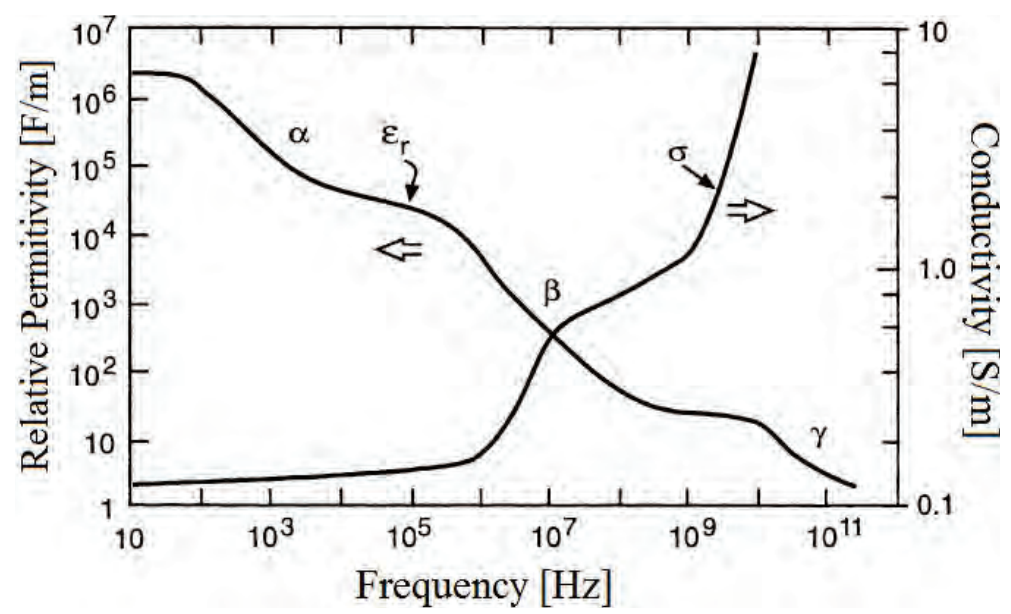

Figure 2.3: Variation of human tissues electrical properties, relative permittivity and conductivity, against frequency. Three dispersion areas of $\alpha, \beta$, and $\gamma$ are characterized in the figure [48].

evident in the alpha dispersion and the permittivity shows a significant decrease $[45]$.

The electrical properties of human tissue are a key element (feature) for designing an energy efficient, low noise, and cost effective IBC transceiver system achieved through the modeling of the human body transmission channel characteristics.

\subsection{Modeling Methods of Body Tissues}

The human body is modeled as a communication channel to investigate the propagation behavior of galvanically or capacitively coupled signals, and hence predict the transmitted data quality. Transmission characteristics of the body have been examined via modeling human body tissues. Although, there is encouraging progress in human body modeling, large discrepancies still exist between empirical results and model predictions. There are different methods for human body channel modeling, including electric equivalent circuit models which are based on parametric model of 
human tissues, numerical simulations such as finite element models (FEM) and finite difference time domain (FDTD) models. Transmission propagation models have been frequently used to guide RF transceiver designs [46], and tissue-specific models are expected to be applied similarly in the IBC field to future IBC transceiver design.

\subsubsection{Human Tissues Parametric Model}

To establish a proper parametric model of tissue properties, dielectric alteration of these properties is determined as a function of frequency. The Cole-Cole equation [47] presents the change of dielectric properties of a tissue over a broad frequency range:

$$
\varepsilon^{*}(\omega)=\varepsilon_{\infty}+\frac{\Delta \varepsilon_{n}}{1+\left(j \omega \tau_{n}\right)^{\left(1-\alpha_{n}\right)}}
$$

where $\epsilon^{*}$ is the complex dielectric constant, $\Delta \epsilon_{n}$ is the magnitude of the dispersion which is calculated from the difference between permittivity at static $\left(\epsilon_{s}\right)$ and infinite frequency $\left(\epsilon_{\infty}\right), \omega$ is the angular frequency, $\tau$ is the relaxation time constant which depends on physical processes such as ion effects, and $\alpha_{n}$ is distribution parameter which is between 0 and 1 . The decrease in the three separate dispersion areas, i.e. alpha, beta, and gamma, is determined by summation of the frequency dependent permittivity expressed by:

$$
\varepsilon^{*}(\omega)=\varepsilon_{\infty}+\sum_{n} \frac{\Delta \varepsilon_{n}}{1+\left(j \omega \tau_{n}\right)^{\left(1-\alpha_{n}\right)}}+\frac{\sigma_{\mathrm{i}}}{j \omega \varepsilon_{0}}
$$

where $\sigma_{i}$ is static ionic conductivity. The dielectric performance of biological tissue is predicted by this summation through proper parameter selection for each tissue. The complex conductivity and the complex specific impedance of tissue are calculated by: 

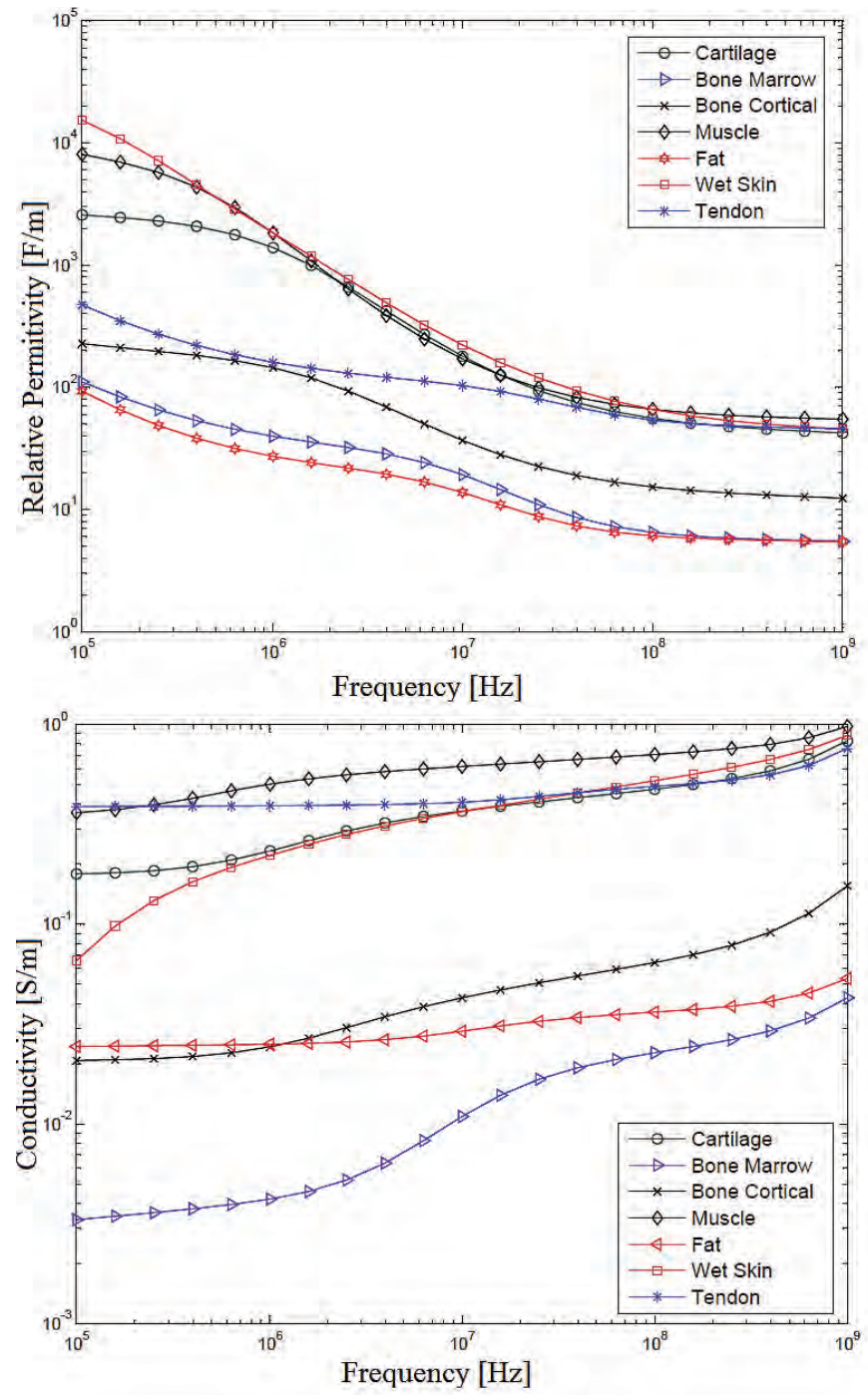

Figure 2.4: Relative permittivity and Conductivity of human body tissues at different frequencies are plotted based on Gabriel et al. research findings.

$$
\sigma^{*}=j \omega \varepsilon_{0} \varepsilon^{*} \quad, \quad \mathrm{z}^{*}=\frac{1}{\sigma^{*}}
$$

The relative permittivity and electrical conductivity of wet skin, fat, muscle, bone cortical, and bone marrow as a function of frequency is shown in figure 2.4. The simulation results are plotted based on equation (2.2) by [1]. 


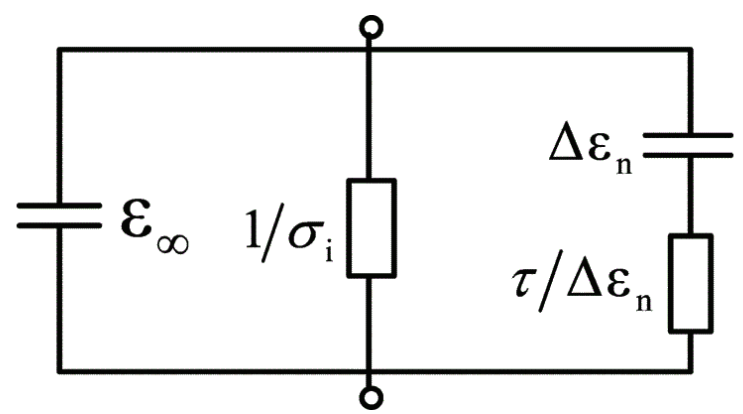

Figure 2.5: Cole-Cole equation equivalent circuit model for a single time constant.

Electrical properties of human body tissues can be modeled by equivalent electrical components such as resistors and capacitors. There are two types of circuit models:

1. $R C$ elements connected in series could be employed to model single limbs and limb linkages [48].

2. A more common method is presenting the circuit model by considering a constant phase element (CPE) with a complex valued impedance given by $Z_{C P E}^{*}=A(j \omega)^{-n}$ where $\mathrm{A}$ is a constant and $\mathrm{n}=\alpha$. This CPE impedance reduces to a simple resistance for $\mathrm{n}=0$ and to a capacitance reactance for $\mathrm{n}=1$. The physical meaning of the CPE is not clearly understood [49]. The model representation of resistive and capacitive elements appears to explain the empirical measurements well. However, determining the exact tissue components responsible for these properties is complicated by the non-homogeneous nature of tissue and randomly distributed cells sizes.

The circuit model of equation (2.2), when $\alpha=0$, is depicted by a parallel combination of an ideal capacitor, a resistor and a CPE which is a series combination of a frequency-dependent capacitance and resistance [50] in figure 2.5. In fact, the Cole-Cole model is mainly applied to biological materials while other distributions 


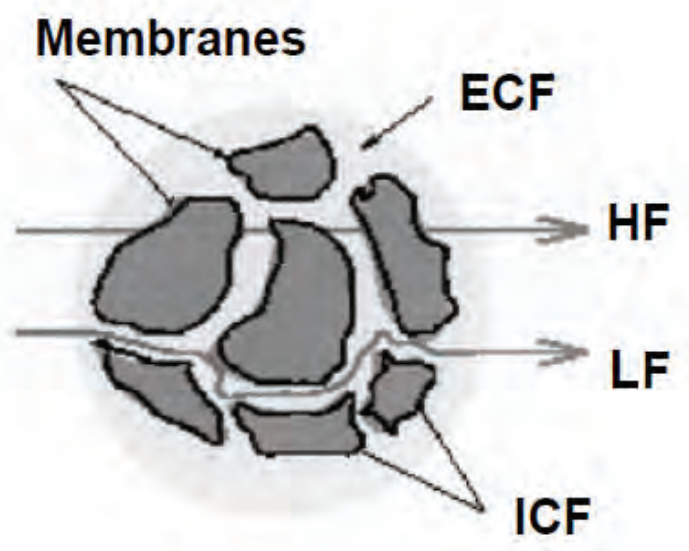

Figure 2.6: Current flow in human body tissues [50].

like Cole - Davidson and Havrilak - Negami are used for non-biological materials [48].

Electrical current flow through human tissue follows several pathways including intra-cellular, extra-cellular, and cellular membrane pathways. The high frequency current tends to pass easily through the tissues (higher conductivity as seen in figure 2.4). The intra-cellular fluid (ICF) is a liquid that circulates inside the cell and surrounded by cell membrane as well as extra-cellular fluid (ECF). The two lines in figure 2.6 show the low frequency (LF) current and high frequency (HF) current pathways [51]. Extra-cellular resistor and capacitor is modeled by $R_{e}$ and $C_{e}$. Constituents of cell membrane and intra-cellular contribution are indicated by $R_{m}$ and $C_{m}$ as well as $R_{i}$ and $C_{i}$ respectively. Kanai et al. in 1987 [52] has presented the complex and simplified circuit model of human tissues seen in figure 2.7. The resistors and capacitors in the proposed model represent physiological effects including blood circulation, metabolism of tissues, and electrolytic concentration of intra- and extra-cellular fluids within the body [52]. 

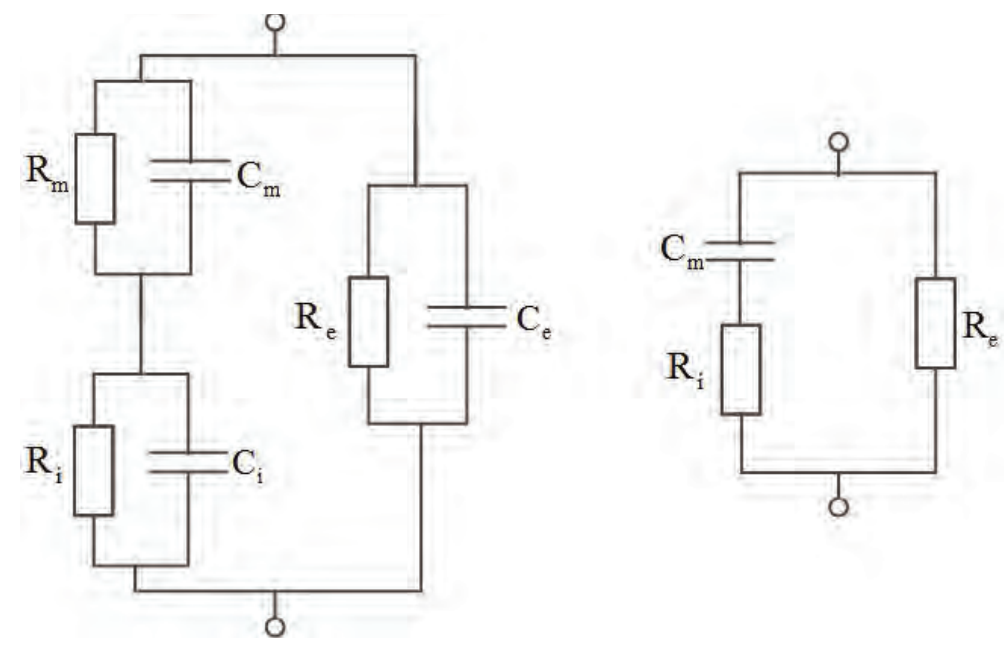

Figure 2.7: Complex (left) and simplified (right) equivalent circuit model of human tissues. The equivalent components of extra-cellular, intra-cellular, and cell membrane are respectively specified by the indexes $e, i$, and $m$ [51].

\subsubsection{Body Channel Circuit Model}

Obtaining the transfer function of the IBC system is the first step for construction of the circuit model. Zimmerman [39] proposed the first circuit model of body communication channel. The model consists of four significant transversal and longitudinal impedances in between transmitter and receiver electrodes. The inter-electrode impedances between the electrodes of the transmitter and receiver were ignored in impedance calculations of his model.

Capacitive coupling frequency characteristics of the human body are identified through a developed $R C$ model in [41]. The body is considered as a single node due to the large impedance of the return path. However, this approximation is not true at high frequency. In this model, human body parts are presented as three cylindrical models which are divided into $R C$ unit blocks each. To measure the $R$ and $C$ quantities the values from Gabriel's research [1] and Zimmerman's circuit model [39] were used. Xu et al. [46] proposed a capacitive coupling channel circuit 
in which they took the body shielding effects into account. Recent examinations [53] also presented skin propagation circuit models obtained from the electro physiological properties of skin. However, the authors did not verify the proposed model through empirical measurements. The comparisons of the model were confirmed only through achieved outcomes from other research data which were performed under different experimental conditions.

In the galvanic coupling circuit model, tissue impedance can be described by the Cole - Cole equation. Hachisuka et al. [54] designed an electric circuit model of galvanic coupled IBC for the first time. They also presented a new two terminal (electrodes) circuit model, where only two of the four electrodes were attached to the body (capacitive coupling). In [54], they suggested four terminals circuit model and used six impedances between transmitter and receiver electrodes. The results indicated that the two terminal electrode structures had a gain of $20 \mathrm{~dB}$ greater than the four terminal electrode structures. Four terminals circuit model with six body tissue impedances and four electrode-skin coupling impedances $\left(Z_{E S}\right)$ were proposed by Wegmueller et al. [9] (see figure 2.8). The contact conditions between the electrodes

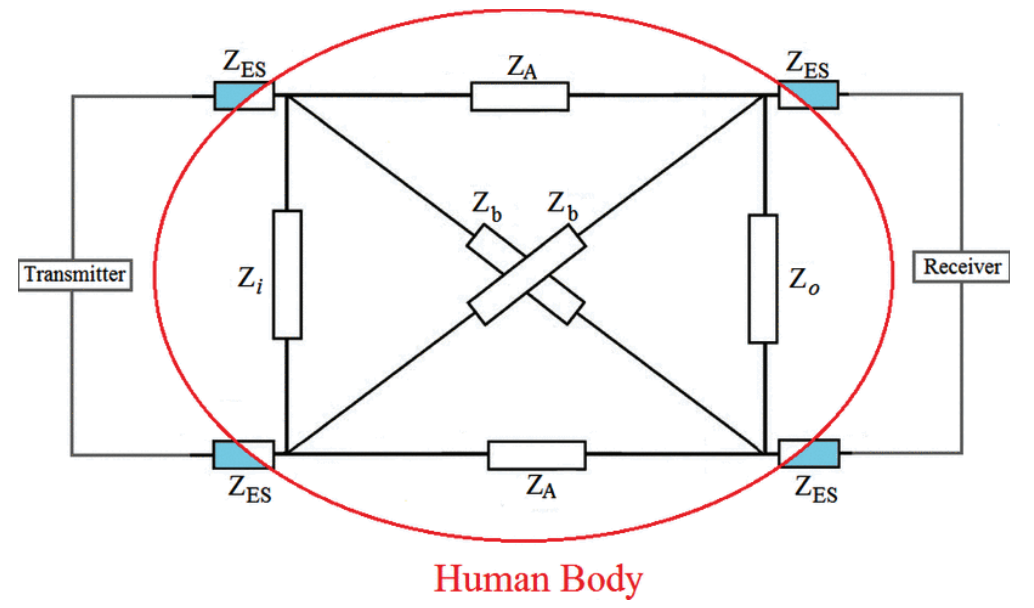

Figure 2.8: Four terminals (electrodes) human arm circuit model during galvanic coupling IBC technique. 
and human body represented by the impedance $\left(Z_{E S}\right)$ influence signal coupling and attenuation as well as the transceiver power consumption [55]. The value of $Z_{E S}$ is represented by a series of three impedances consisting of electrode impedance, interface impedance (gel impedance), and skin layer impedances which include epidermis and subdermal impedances. According to Besio et al. [56], good contact between electrode and skin surface is established when $Z_{E S}<10 \mathrm{k} \Omega$. The output resistance of the transmitter and the input resistance of the receiver were considered by Song et al. in a four terminal circuit model [57]. In the mentioned models, the geometry of the human body is approximated as a homogeneous solid volume. More detailed components such as joints were not considered in the circuit model. A limitation of this technique is that model complexity increases dramatically with the number of body tissue layers.

\subsubsection{Finite Element Model (FEM)}

FEM is a technique which could model individual body tissues. It is based on the numerical solutions of partial differential equations and integrals. Electrical behavior of the human body is simulated through a physical model using this technique. The FEM is intended for better investigation of human anatomy effects on signal transmission in the IBC method. It is also able to reconstruct the potential distributions caused by induced current into the human tissue.

$\mathrm{Xu}$ et al. [58] have utilized FEM to investigate capacitive coupled IBC for the first time. The environment around the human body was separated into three different regions: near-field region, transmission region, and far-field region. Arm, chest, abdomen, and leg formed the four parts of the model. The results of FEM simulation showed that the presence of capacitive return path in capacitive coupled IBC plays 
a pivotal role in determining the characteristics of the body communication channel such as channel loss. It was also indicated that the return path is mainly coupled through the external ground during the capacitive IBC method.

The signal attenuation through the body in galvanic coupling IBC was investigated using FEM [59]. Authors explored the bioimpedance, electric field, current density, and the influence of channel length and the inter-electrode distance on signal propagation through the body in this work. They employed the default meshing option provided by COMSOL Multiphysics to model the arm geometry of the human body. While the model simulation results showed that an increase in the channel length led to higher signal attenuation, increasing the inter-electrode distance had a considerable decrement of about $1.0 \mathrm{~dB} / \mathrm{cm}$ in attenuation. In general, there was good agreement between simulation and empirical results over the investigated frequency range of $1.0 \mathrm{k}-100 \mathrm{MHz}$.

However, a major drawback with this method is the large size and the complexity of the human body which can significantly reduce the simulation accuracy [57]. Among different methods for channel modeling, the equivalent circuit model contains a few number of elements. However, the FEM is created from arbitrary number of nodes (more than thousands) to construct only one mesh. According to Wegmueller [6], to model the body channel using the FEM, mesh sizes were between 150000 and 200000 elements. Therefore, employing the circuit model could reduce the complexity as well as the analysis duration.

\subsubsection{Circuit-Coupled FEM Model}

Circuit-coupled FEM provides useful insights into the capacitive coupled IBC. It allows modeling of IBC channel components with different abstraction levels. The 


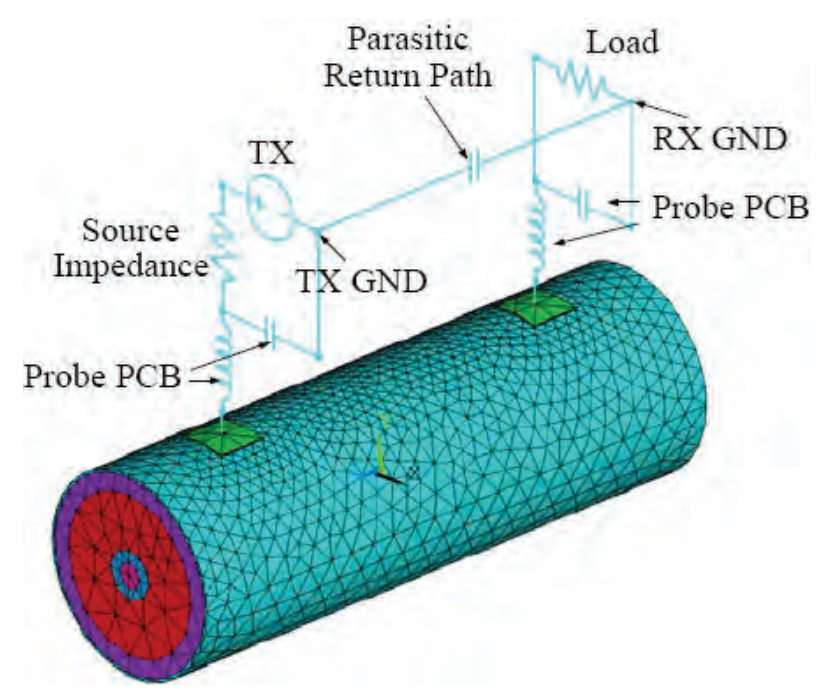

Figure 2.9: Human forearm model is simulated using the circuit-coupled FEM method. The parasitic return path is represented by mean of a capacitor element [57].

human forearm was modeled by a multi-layer FEM and the parasitic effects of probe PCBs (printed circuit board) were modeled by LC circuits (figure 2.9 [58]). Since frequency-dependent features of the parasitic return path make it difficult to simulate, the authors used a simplified capacitor model for the parasitic return path [58]. The circuit-coupled FEM revealed that large distance between transmitter and receiver electrodes led to higher signal attenuation. The variation of distance between transmitter and receiver was thought to be mainly caused by the size of the parasitic capacitor return path.

\subsubsection{FDTD Model}

Finite difference time domain (FDTD) model is a computational modeling technique used in the field of electromagnetism. It examines the distribution of the electric field inside and outside the complex geometries such as human body. Since the model is 
merely operating in the presence of electromagnetic fields in the simulation area, it is considered as the state-of-the-art method for investigation of signal behavior in IBC technology.

The arm was modeled by FDTD calculation model through the capacitive coupling method by Fujii et al. [60]. Authors believed that a simple homogeneous calculation model was sufficient for the capacitive coupled IBC. It was concluded that the ground electrode in the transmitter side was necessary to strengthen the electric field around the limb. However, FDTD is a time consuming process for constructing a model in the lower frequency range and more suitable for higher frequency ranges (several hundred megahertz [61]) which is outside our current range of interest.

\subsubsection{Theoretical Electromagnetic Model}

Theoretical models of human body channels can be developed by solving Maxwell's equations and specific boundary conditions. Maxwell's equations explain the coupling between electromagnetic signals around the body and body itself through a set of complete electric field equations. Recently, a theoretical model of the capacitive IBC using Maxwell's equations was proposed by Bae et al. [62] to predict the properties of the electrical wave travelling on the surface of the body. The complete equation of electric field around the body consists of three electric field components; the quasi-static near-field, induction-field radiation, and the surface wave far-field, were considered to obtain the general IBC model. The results from both measurement and proposed theoretical model indicated that increase in channel length led to channel path loss enhancement. Their model was empirically verified for operating frequencies of $0.1-100 \mathrm{MHz}$ and channel lengths of up to $1.3 \mathrm{~m}$.

Chen et al. [63] proposed a galvanic coupling electromagnetic model to predict 
the effects of the body channel loss opposed to body tissue (muscle) thickness in both IBC and RF techniques. The transmitter and receiver were supposed as implantable and wearable sensors, respectively. When the implanted sensors were $60 \mathrm{~mm}$ deep from the body surface, simulation results using COMSOL predicted $35 \mathrm{~dB}$ and $50 \mathrm{~dB}$ signal attenuation for IBC and RF techniques, respectively. This difference increased to $20 \mathrm{~dB}$ for a transmitter-receiver distance of $80 \mathrm{~mm}$. The results also demonstrated that galvanic coupling IBC is more power efficient than RF for deeper implants.

\subsection{IBC Transceiver Design}

In communication systems design several challenges need to be addressed. Channel characteristics are the main challenge for an ideal communication system design. Typically, transmitter, communication channel, and receiver comprise three fundamental stages of any communication system. The transmitter is composed of several sub systems: an analog-to-digital converter (ADC) an encoder, and a modulator. Likewise, the receiver may include a demodulator, a decoder, and a digital-to-analog converter (DAC). A communication channel refers to a physical transmission path which allows the propagation of the signal. It determines the technique to be used in real communications. The block diagram of a general IBC transceiver system is depicted in figure 2.10 .

A communication channel functions relatively like a filter that attenuates the signal and causes transmission signal loss and distortion. The channel distance affects the signal attenuation, where larger distances result in more attenuation. Furthermore, frequency dependent gain characteristics and multipath effects cause transmission wave shape distortion. These phenomena necessitate deeper understanding of 
the transmission medium to design more effective IBC transceivers [64].

The maximum efficiency of a transceiver is determined by its compatibility within the network [65]. Examples of transceiver design parameters are: data rate (number of bits per second), sensitivity which is the minimum signal power required to receive data correctly, transmitter output power, communication interface, carrier operation frequency, or the range of signal that can be sent and received, measurement resolution, and maximum transmission distance. Measurement resolution determines the smallest digital resolution while maximum transmission distance is the largest possible distance of the transmitter and receiver. Extra factors affecting the choice of transceivers involve power source, supply voltage, supply current, transmitter inputs, receiver inputs, and RF connector types. Uniquely designed transceivers will reduce the complexity and will offer the ability to fully integrate the whole system into a more compact form [43].

While a distinctive feature of IBC is to design efficient hardware transceivers, the hardware complexity of units including size, power, and cost must be minimized [66]. However, there is no definite principle to obtain the best electronic design of an IBC system. Power consumption, data rate, carrier frequency, and modulation method

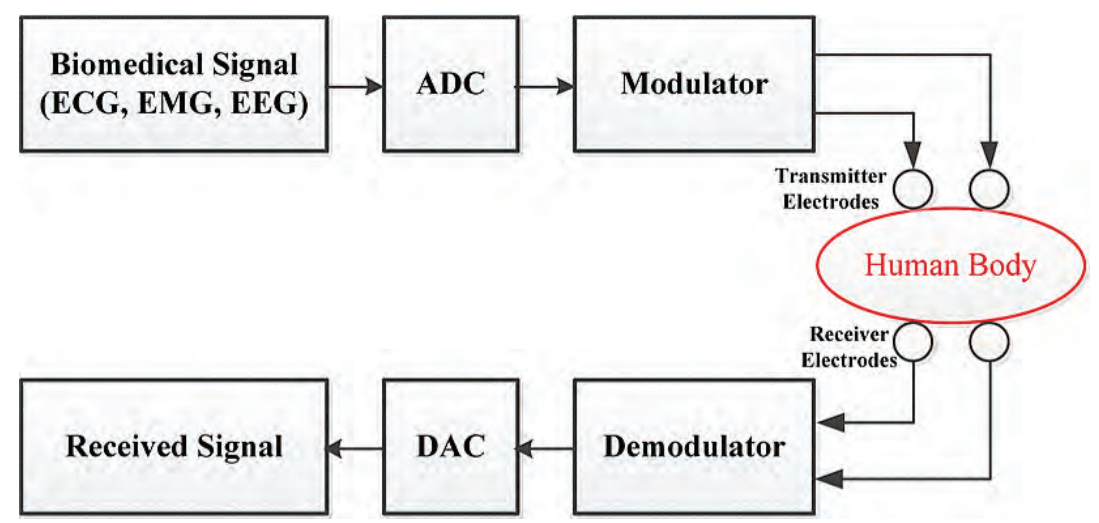

Figure 2.10: Simplified block diagram of the IBC transceiver system. 
are the main concerns [9]. Therefore, the IBC transceiver should be simple, have full integration ability, consume low power, and have the ability to transmit at low power. Since power hungry transceiver nodes need large batteries, power sources like solar cells appear to be suitable for IBC. At the same time, low voltage operation is required for IBC due to health and safety reasons e.g. direct contact with the human body $[67]$.

Several IBC transceiver designs have been proposed based on capacitive and galvanic coupling approaches. However, no acceptable standard has been established to implement an optimal design of a full intra-body transceiver system in terms of carrier frequency, modulation scheme, data rate, and power consumption. In the following, a survey of some recent transceiver designs is highlighted.

A battery powered transmitter and receiver unit was developed in the first IBC system prototype PAN (personal area network) transceiver [68]. Results indicated that the best received signal magnitude could be obtained when the PAN devices were placed on the feet close to the physical ground. Two kinds of digital modulation, on-off keying (OOK) and direct sequence spread spectrum (DSSS) techniques were examined in the scheme. In OOK, the existence of the carrier produces a binary one, while binary zero is represented by switching off the carrier. DSSS modulates the carrier signal with pseudo-noise (PN) sequences which are widely used in digital communications. The whole PN sequence is transmitted when a message bit is one, while it is inverted when a message bit is zero. To extract the message at the receiver, the transmitter and receiver PN sequence must be synchronized which is the greatest challenge of a DSSS system. Unsurprisingly, the OOK was found to be more effective and easier to implement for the PAN transceiver. The optimum carrier frequency range was determined between $100 \mathrm{kHz}$ to $500 \mathrm{kHz}$ and a suitable data rate was 
considered to be $2.4 \mathrm{kbps}$. However, the data rate was not practical for sensors sending high streams of data. The transceiver circuit of capacitive coupling IBC system was designed and implemented by Partridge et al. [69]. They extended Zimmerman's original IBC system by adding filters and amplifiers to the transceiver circuit. Two different microcontrollers were used in the circuit to generate and modulate the input digital signal. In addition, they utilized a frequency-shift keying (FSK) detector in the proposed circuit achieving a data rate of $38.4 \mathrm{kbps}$. The changes of frequency when message signal switch from zero to one or vice versa in FSK scheme leads to transmission of a large amount of data in a narrow bandwidth. Since complex body area networks such as those using portable electroencephalogram (EEG) 192-channel recordings [9], need data rates of almost hundreds of kbps, the achieved data rate was barely sufficient for continuous monitoring. To compensate the low data rate of an IBC transceiver in such a network, a larger array of sensors is required for increased redundancy which leads to increased power consumption at the same time. The low power consumption and high data rate (2.0 Mbps) capacitive coupled IBC transceiver based on wideband signaling (WBS) communication method was introduced in [70]. The WBS scheme directly transmits binary digital signals through wideband pulse signals. Lin et al. [67] implemented the small size system on a chip (SOC) IBC system for biomedical applications for the first time. The authors believed that the suitable carrier frequency for IBC was $200 \mathrm{MHz}$ and data rates up to $2 \mathrm{Mbps}$ were reported for OOK modulation. Moreover, they proposed a new generation of transceiver which required less than $0.5 \mathrm{~V}$ making it suitable for use with solar cell power. Unfortunately, this system had a short channel length or transmission distance (from subject's wrist to forearm). Recently, Xu et al. [22] inspected the systematic features of the electric field IBC (EF-IBC) channel containing attenuation, noise, and distortion by 
making use of battery powered transceiver board. Two modulation methods of binary phase shift keying (BPSK) and quadrature PSK (QPSK) was examined and a high speed 10Mbps EF-IBC links was created. A microcontroller unit was used in transceiver board to generate the baseband in-phase and quadrature digital signals and also controlled the digital direct synthesizer (DDS). Various measurements with different received signal powers and symbol rates indicated that the channel noise was white Gaussian. It was also concluded that both the signal-dependent distortion and the signal independent noise influenced the channel signal-to-noise ratio (SNR). Considering the bit error rate (BER) as a modulation scheme metric, it was revealed that QPSK has the highest transmission power at both $10^{-3}$ and $10^{-6}$ BER. A recent suggested capacitive coupling IBC transceiver by Bae et al. [21] was able to fulfill the requirements of the WBAN standard, such as network coexistence and quality of service (QoS) scalability, at the $10 \mathrm{Mbps}$ data rate in the operating frequency range of 40-120 MHz. This energy efficient transceiver was combined with contact impedance sensing (CIS), a ring oscillator as well as the double-FSK modulation scheme which decreased the power consumption of the transmitter and receiver to 2.0 and $2.4 \mathrm{~mW}$, respectively.

The principles of galvanic coupling were first introduced by Oberle [40] while designing a single chip low power biomedical system. The continuous phase frequency shift keying (CPFSK) modulation scheme was used in his implementation. A waveguide measurement approach was first performed by Hachisuka et al. [54], [61] to confirm the possibility of IBC design and to analyze the transmission characteristics of the human body. The various electrode sizes and materials as well as an ideal carrier frequency range were specifically taken into account. Transmission devices using 
10.7 MHz FSK at 9.6 kbps, and 10.7 MHz FM were developed for digital and analog transmission respectively. A suitable carrier frequency range of between $10 \mathrm{MHz}$ to $50 \mathrm{MHz}$ was considered. However, the proposed transceiver board in this study suffered from low data communication rate, low integration level, and large form factor. In further experiments on the galvanic approach by Wegmueller et al. [71], [72], the human body characteristics were taken into account and models were generated for static position of human body. In practical measurements, two FSK and BPSK digital modulation types were applied with a $128 \mathrm{kbps}$ and $255 \mathrm{kbps}$ data transfer rate respectively. A battery powered transceiver was also employed in order to isolate the sensor units from other power line. A field-programmable gate array (FPGA) was used to provide interfaces between analog front-end and digital communication link. However, the achieved data rate of transceiver was low for some biomedical applications such as medical implant communications service (MICS). An IBC/MICS dual modes transceiver was designed and fabricated by Cho et al. to communicate with both on-body and implanted sensors [73]. The operation frequency range was considered 30 to $70 \mathrm{MHz}$ and 402 to $405 \mathrm{MHz}$ for IBC and MICS, respectively. The achieved data rate of system was 5 Mbps for IBC and $200 \mathrm{kbps}$ for MICS transceiver. Since the common front-end circuits including amplifier and mixer was employed in both IBC and MICS receiver, therefore the total power consumption of system was reduced to $10.8 \mathrm{~mW}$. A list of some current transceivers based on both capacitive and galvanic coupling IBC is shown in Table 2.2.

In 2009, Al-Ashmouny et al. [74] applied the IBC method using brain tissue as a communication medium calling it intra-brain communication. Two miniaturized communication chips were designed and implanted in a rat brain. BFSK modulation technique was used in the proposed system with frequencies of 100 to $400 \mathrm{kHz}$. While 
Table 2.2: Summary and Comparision of Currently Reported IBC Transceivers

\begin{tabular}{|c|c|c|c|c|c|}
\hline & $\begin{array}{l}\text { Coupling } \\
\text { Method }\end{array}$ & $\begin{array}{l}\text { Carrier } \\
\text { Frequency }\end{array}$ & $\begin{array}{l}\text { Modulation } \\
\text { Technique }\end{array}$ & $\begin{array}{l}\text { Data } \\
\text { Rate }\end{array}$ & $\begin{array}{l}\text { Power Con- } \\
\text { sumption }\end{array}$ \\
\hline \hline$[68]$ & Capacitive & $330 \mathrm{kHz}$ & OOK & $\begin{array}{l}2.4 \\
\mathrm{kbps}\end{array}$ & $400 \mathrm{~mW}$ \\
\hline$[69]$ & Capacitive & $160 \mathrm{kHz}$ & FSK & $\begin{array}{l}38.4 \\
\mathrm{kbps}\end{array}$ & Not reported \\
\hline$[61]$ & Capacitive & $10.7 \mathrm{MHz}$ & FSK & $\begin{array}{l}9.6 \\
\mathrm{kbps}\end{array}$ & Not reported \\
\hline$[70]$ & Capacitive & $1.0-200$ & Not & $\begin{array}{l}2.0 \\
\text { Mbps }\end{array}$ & $2.4 \mathrm{~mW}$ \\
\hline$[67]$ & Capacitive & $200 \mathrm{MHz}$ & OOK & $\begin{array}{l}2.0 \\
\mathrm{Mbps}\end{array}$ & $4.535 \mathrm{~mW}$ \\
\hline$[22]$ & Capacitive & $20-100$ & PSK & $\begin{array}{l}10 \\
\mathrm{Mbps}\end{array}$ & Not reported \\
\hline$[21]$ & Capacitive & $40-120$ & $\begin{array}{l}\text { Double- } \\
\text { FSK }\end{array}$ & $\begin{array}{l}10 \\
\text { Mbps }\end{array}$ & $4.4 \mathrm{~mW}$ \\
\hline$[40]$ & Galvanic & $60 \mathrm{kHz}$ & CPFSK & $\begin{array}{l}4.8 \\
\mathrm{kbps}\end{array}$ & Not reported \\
\hline$[9]$ & Galvanic & $256 \mathrm{kHz}$ & BPSK & $\begin{array}{l}64 \\
\mathrm{kbps}\end{array}$ & $726 \mathrm{~mW}$ \\
\hline
\end{tabular}

the distance between electrodes was $15 \mathrm{~mm}$, the energy consumption was less than 650 pJ per transmitted bit. Similarly recent research on wireless intra-brain communication has also transmitted data through a rat brain [75]. Authors designed the small CMOS chip $(550 \mu \mathrm{m} \times 700 \mu \mathrm{m})$ to successfully transmit a $50 \mathrm{MHz}$ AM modulated signal using a $3.3 \mathrm{~V}$ supply voltage.

\subsection{IBC Challenges}

Studies in the literature have investigated the IBC technique from different perspectives. These aspects include diverse methods to measure the IBC characteristics, various experimental condition, different kinds of modeling, and several methods to 
design the efficient IBC system. However, despite the considerable and divergent amount of works on IBC and despite the fact that IBC has been ratified in the WBAN standard, there are still challenges to be addressed. For instance, the effect of IBC user motion on transmission quality is one of the main issues which need to be deal with. The influence of static and dynamic body situations is an influencing element since the IBC system needs to continuously keep track of the patients' or athletes' vital signs.

At the same time, the increasing data rate for low frequency carriers is needed in order to develop an energy efficient IBC system. IBC standard postulates the maximum data rate of $1321.5 \mathrm{kbps}$ in the $21 \mathrm{MHz}$ however, the results fulfills only the static body condition during the capacitive coupled IBC. This study investigates the effects of body motions during galvanic and capacitive IBC methods within a wider frequency range (up to $200 \mathrm{MHz}$ ) compared to previous researches. It also pursues to achieve a suitable frequency range for IBC during body movement.

We examine a digital baseband signal traveling through the body and propose a new energy efficient IBC system applying the galvanic coupling IBC method.

\subsection{Summary}

The present chapter provided a deep survey of research in IBC method. IBC is a short range non-RF wireless communication technique specified by the IEEE 802.15.6 using the human body as a transmission medium. IBC is a recent and novel communication technique and research studies in this field are in progress so as to address the many challenges and issues. In this chapter, we reviewed the current IBC coupling methods, various IBC models, and latest transceiver designs. As it stands, the IBC 
technique potentially offers a more power efficient and naturally secure short range communication method for body sensor networks, compared to wireless RF.

In the next chapters, IBC characterizations based on empirical measurement will be investigated comprehensively at the higher frequency range compared to past studies. Since there was a dearth of empirical studies on the signal propagation through dynamic body, we aim to examine the body motion effects on IBC through the next chapter. Moreover, new body channel model will be proposed considering the limb joints effect in this dissertation. Finally, a new energy efficient IBC transceiver will be designed and implemented on FPGA. The transceiver adapts PPM modulation with high data throughput for WBAN applications. 


\section{Chapter 3}

\section{Experimental Methodology}

Recent researches on IBC have investigated the influence of body movement on signal propagation through the body channel. These studies have assessed the general and complex movements such as moving the arm up and down $[11,78]$. Yet, none of these studies have addressed the IBC characteristics with respect to body limb motion details. Since limb joints control human mobility, the impact of the elbow and knee joints within the signal path as well as the effects of joint flexion and extension on signal attenuation is examined in our research. To this end, some real measurements need to be conducted to confirm the achieved results from the proposed model simulations before device fabrication begins. These findings will aid to improve the efficiency of IBC system which is important for BAN applications. It is worth noting that, the proposed experimental setups will be applied for investigations of the body channel characteristics in chapter 4 and 5. This Chapter is organized as follows. Section 3.1 will discuss the required conditions to perform acceptable measurements in terms of safety and technical requirements. Section 3.2 presents a framework of the 
various types of IBC measurements used in the thesis. Finally, in the last section (section 3.3), we will summarize the key issues and considerations of IBC measurement architecture.

\subsection{Pre-measurement Preparation and Safety}

In general terms, any communication system is composed of three fundamental components that are, a transmitter, the communication channel, and a receiver. Since the communication channel of IBC technique is the human body, safety and medical regulations have to be fulfilled for any real measurement on a human subject. In this work, ethics approval (number: HRETH 11/135) was obtained from Victoria University Human Research Ethics Committee (VUHREC) as a first step prior to performing empirical measurements on the human body.

Overall, the IBC empirical experiment poses two main possible health risks to the human body namely, physical risk and the danger of electrical shock. First, physical risk refers to the physical pain and discomfort caused by removing the on-body electrodes from the body fragments of electrode gel. Therefore, it is required to remove the hair from that particular skin surface. Shaving the target skin surface not only relieves the pain that may have been caused by sudden removal of electrodes, but also leads to acquisition of signals with higher quality by reducing contact impedance of the electrode-skin [86]. Second, faulty measurement instruments may create electrical hazards and accordingly increase the risk of electrical shock. The induced current intensity at specific frequencies determines the intensity of the electrical shock through the body [79]. For instance the maximum harmless induced current for IBC should be $20 \mathrm{~mA}$ at frequency range of $100 \mathrm{kHz}$ to $110 \mathrm{MHz}$ (general public exposure) [80]. 


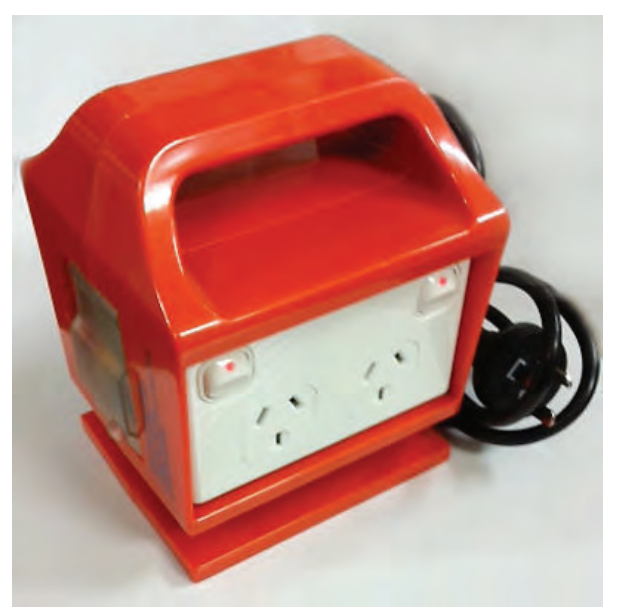

Figure 3.1: Portable residual-current device (RCD) safety switch power outlet.

During our experiments, to prevent any possible risks, the measurement equipment will be connected to the power line (50 Hz mains) via a portable "medical grade Residual-Current Device" (RCD) safety switch power outlet (see figure 3.1). The device protects both human subjects and the measurement appliances during any lab experiments that may be potentially hazardous. In other experiments, we use a battery powered transmitter and receiver board with less current draw and hence minimize the risk of electric shocks further.

\subsection{Measurement Setup}

In this thesis, we carried out empirical measurements to demonstrate the effect of electrical signal transmission through the human body using IBC technique. Figure 3.2 shows the measurement setup as applied in IBC system. Three main segments of IBC system are on-body surface electrodes, human body as device under test (DUT), and electronic equipment such as transmitter and receiver. 


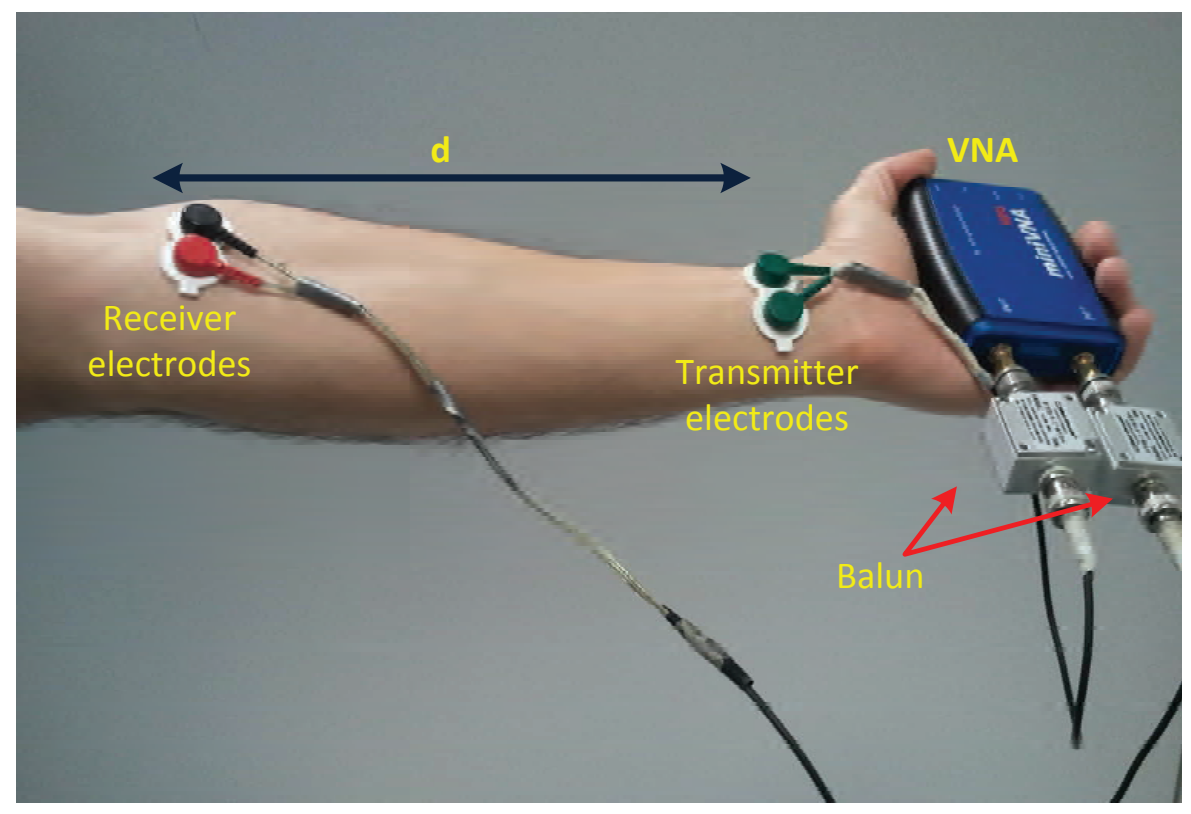

Figure 3.2: The dedicated measurement setup in IBC method.

\subsubsection{On-Body Electrodes}

During the measurement, each subject is equipped with two pair of hypoallergenic surface electrodes in transmitter and receiver sides. The electrodes are utilized as communication interface between electronic devices (transmitter and receiver) and the body skin. Using suitable impedance matched materials should reduce the dielectric mismatch between connecting cables and the human body. In our measurement, both single and dual commercial silver/silver chloride $(\mathrm{Ag} / \mathrm{AgCl})$ electrode (Noraxon Inc., Scottsdale, AZ, USA) was employed. These electrodes are popular in bio-potential systems such as electromyography (EMG) systems. Since the dry skin is a poor conductor and creates high electrode-body impedance up to $20 \mathrm{k} \Omega$ [79], these pre-gelled electrodes moisten the skin and provide better match to the minimized input skin impedance $(1 \mathrm{k} \Omega)$ for maximum signal acquisition. Additionally, employing the self-adhesiveness of Noraxon electrodes keeps them stable during the human 


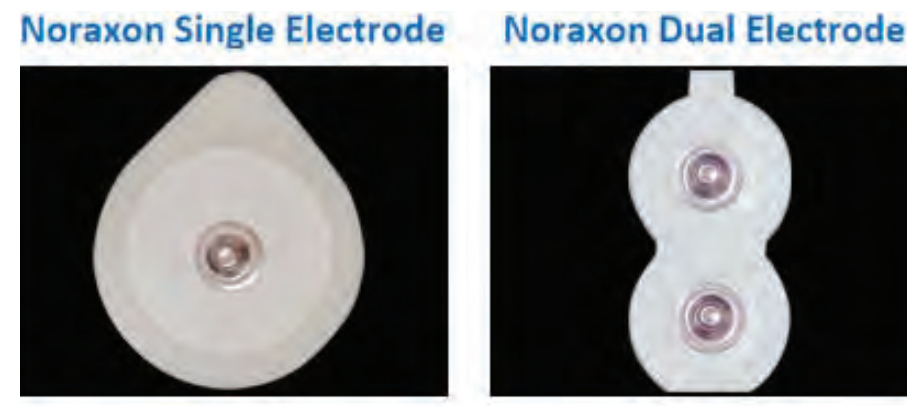

Figure 3.3: The employed electrodes in IBC measurement system.

subject movement. Figure 3.3 shows two different Noraxon electrodes employed in our study. Since both electrodes and leads were commercial equipment employed in EMG applications, the possibility of any leakage and interference from the separation points of the electrodes and leads is negligible (in low frequencies).

According to [81], the $\mathrm{Ag} / \mathrm{AgCl}$ electrodes demonstrate the lower noise power spectrum density (PSD) $\left(8.7 \times 10^{-9} V^{2} / H z\right)$ compared to textile (fabric based) electrodes $\left(1.0 \times 10^{-6} V^{2} / H z\right.$ for polyethylene terephthalate $(\mathrm{PET})-\mathrm{Cu}-\mathrm{Ni}$ fabric) which are suitable for wearable networks in dynamic BANs. Due to the higher electrical conductivity of $\mathrm{Ag}$ compared to copper $(\mathrm{Cu})$ (around 6\%), it is most appropriate compared to $\mathrm{Cu}$ in long-term healthcare monitoring. It is well worth to emphasize that the quick corrosion of $\mathrm{Cu}$ may lead to the achievement of unreliable results and biocompatibility issues. Another influential element in the structure of electrode is the effective electrode area (EEA). The EEA is the circular conductive area of the electrode which is in direct contact with skin through the electrode gel (see figure 3.4) [50]. The radius of EEA is equal to $1.0 \mathrm{~cm}$ in our Noraxon electrodes. 


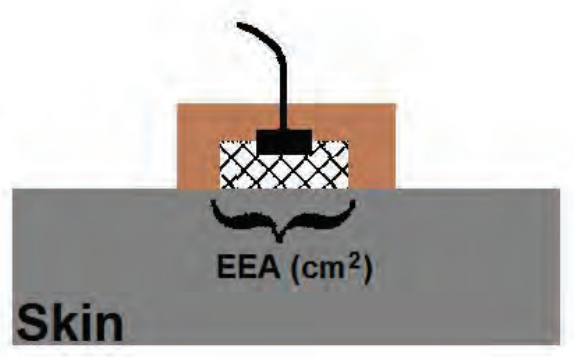

Insulator

Metal

Gel

Figure 3.4: Skin surface electrode designs including metal, gel, and insulator of electrode.

\subsubsection{Human Body Channel}

The IBC is distinguished from other communication techniques due to the two distinctive features of the human body. First, the human body is a non-static communication channel which undergoes high level of movements during the day. This leads us to be confronted with variations of signal power while performing out measurements. Second, human body has a non-homogeneous biological structure. Different features of human bones, muscle, and adipose tissue (fat) could potentially affect the signal transmission. In order to deal with the non-static features of the human body and to deeply and accurately characterize the body channel, we need to be aware of the amount of signal propagation. These can be done by measuring scattering parameters (S-parameters) that describe the operation of a two-port circuit network [82]. The S-parameters describe the operation of a two-port circuit network. As shown in figure 3.6, the two port circuit network is composed of transmitter electrodes, human body as a transmission medium, and receiver electrodes. Reflected signal power is measured by $\left(S_{11}\right)$ and $\left(S_{22}\right)$, known as reflection coefficients in the transmitter and receiver sides, respectively. $\left(S_{12}\right)$ and $\left(S_{21}\right)$ refer to signal transmission from transmitter electrodes to received electrodes, and are known as reverse and forward 


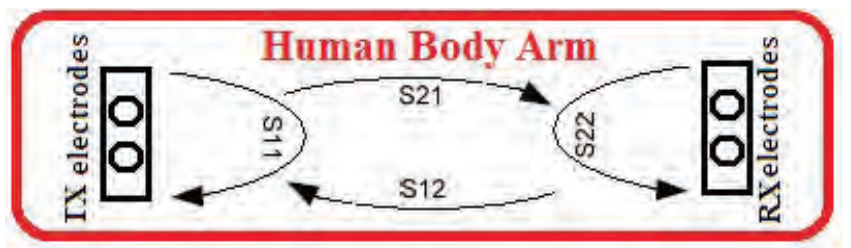

Figure 3.5: IBC measurement setup associated with S-parameter.

transmission coefficients, respectively. Although, the actual attenuation of the propagated signal through the body is measured by $\left(S_{21}\right)$, the amount of reflected signals (especially the $\left(S_{11}\right)$ ) could represent an extra loss and vary the value of $\left(S_{21}\right)$. Using the commercial electrodes in both transmitter and receiver sides and the calibration of VNA prior to measurements could minimize the reflected signal effects.

\subsubsection{Electronic Equipment}

To measure S-parameters, a two port vector network analyzer (VNA) is usually employed. The VNA measures the magnitude and phase of the network particularly at radio frequency $(\mathrm{RF})$ ranges. The characteristics of two-port networks such as attenuators, filters, or the human body in our study, could be measured using the VNA. It could be also used to characterize single-port networks such as passive components. As seen in figure 3.2 a portable battery-powered MiniVNA Pro (Mini Radio Solution Inc., Poland) was used in this study. Using battery-powered measurement equipment limits the noise and interference which are generated by the main power lines (50 $\mathrm{Hz}$ ) [83]. Additionally, the light weight portable miniVNA Pro (120 g compared to more than $2.7 \mathrm{~kg}$ for typical handheld VNAs, such as Agilent N9923A FieldFox, USA) makes it suitable for the outdoor measurements as well as for empirical measurements on non-static human body. Port 1 of MiniVNA Pro (DUT) generates the induced sinusoid signal and feeds into the human body which is the device under test (DUT) 


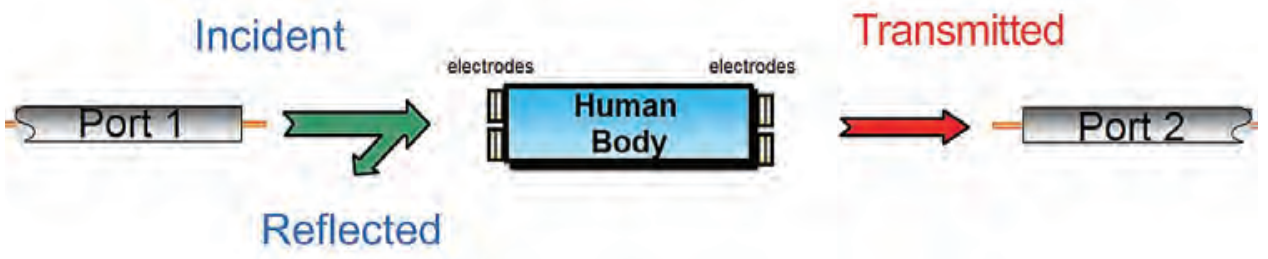

Figure 3.6: Measuring the transmission and reflection signal using MiniVNA Pro while the human body acts as DUT.

in our research. Detector port (DET) of MiniVNA Pro, Port 2, will be considered as receiver. The method of measuring the transmitted signal through the body by means of MiniVNA is shown in figure 3.6. To calculate the transmission coefficient $\left(S_{21}\right)$, equation (3.1) is applied as follows:

$$
S_{21}=20 \log _{10}\left(\frac{V_{\text {transmitted }}}{V_{\text {incident }}}\right)
$$

The value of $\left(S_{21}\right)$ (transmission loss or channel gain) is described in decibel (dB). Since the human body channel (DUT) represents a considerable loss during the signal transmission (more than $60 \mathrm{~dB}[18]$ ), the value of $S_{21}$ will be negative in our study. The MiniVNA Pro was calibrated using standard calibration kits (short, open, and load) to ensure accuracy with a data acquisition rate of 2000 samples per measurement. The MiniVNA Pro was periodically recalibrated to transmit low power test signal to human body for further safety. The output power of transmitter port was fixed to a very lower power of just $0 \mathrm{dBm}(=1.0 \mathrm{~mW})$. According to the exposure guidelines of the "international commission on non-ionizing radiation protection" (ICNIRP), this signal power is 20 times below the maximum allowed input signal power to human body, i.e. the safety limit [84]. In addition, the input signal power was selected based 


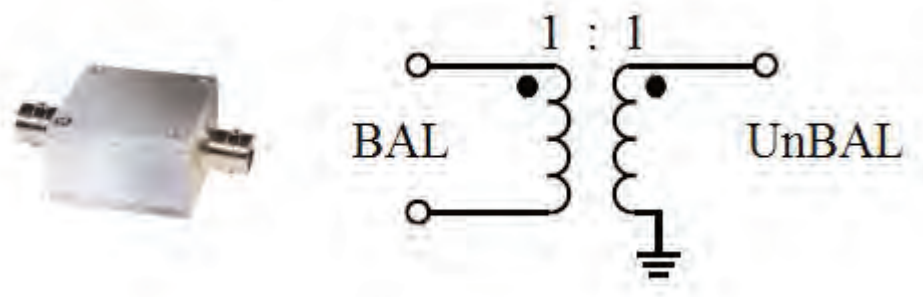

Figure 3.7: The schematic diagram of the employed baluns in the measurement setup.

on the study of "world health organization" (WHO) on the possible health effects of exposure to electromagnetic field [85].

To eliminate the undesired common mode signals from power line interference as well as to decouple both transmitter and receiver from MiniVNA Pro, differential amplifiers or balun (balance-unbalanced) transformers could be employed. They prevented the signal return path from being shorted to the common voltage of the MiniVNA battery. The two baluns (FTB-1-1+, mini-Circuits Inc., Brooklyn, NY, USA) used in measurements setup in this study are shown in figure 3.2. Since two ports of the same miniVNA Pro act as transmitter and receiver, the pair of baluns decouples the ports from each other. They also transform a single-ended (unbalanced) signal into a differential (balanced) signal which is required for the galvanic coupling IBC. Figure 3.7 shows the schematic diagram of the coaxial balun transformers which cover the wide frequency band of 0.2-500 MHz. Moreover, since the presence of baluns can affect the channel response, the measured transmission coefficient of the balun is demonstrated in figure 3.8. The overall picture of the IBC measurement architectures in this study, are depicted in figure 3.9. As mentioned earlier in section 3.2, the system is composed of the human body, the surface electrodes, portable MiniVNA Pro, balun transformers which are placed between on-body electrodes and MiniVNA Pro ports, and connecting cables. It should be noted that the measurement frequency range of 
this study, 0.3-200 MHz, is higher than the recent studies which covered only a limited frequency range for both capacitive (up to $100 \mathrm{MHz}[27,78]$ ) and galvanic coupling method (less than 2.0 MHz [27]). Hence, we propose to refer to the measurement setups as a "two-electrode configuration" and "four-electrode configuration" instead of IBC capacitive and galvanic coupling methods, respectively.

Before we proceed with the overall experiments on human body, it is required to first, determine any possible influence that the use of baluns might have on the achieved results and second, to investigate antenna effects of both body and connecting wires on the measurement.

We measure the $S_{21}$ of human body channel with and without employing the balun transformers in the measurement setup to determine the presence of baluns effects on IBC results (see figure 3.10). In this measurement the on-body communication channel length was fixed to $20 \mathrm{~cm}$. In the experiment without baluns, the connecting

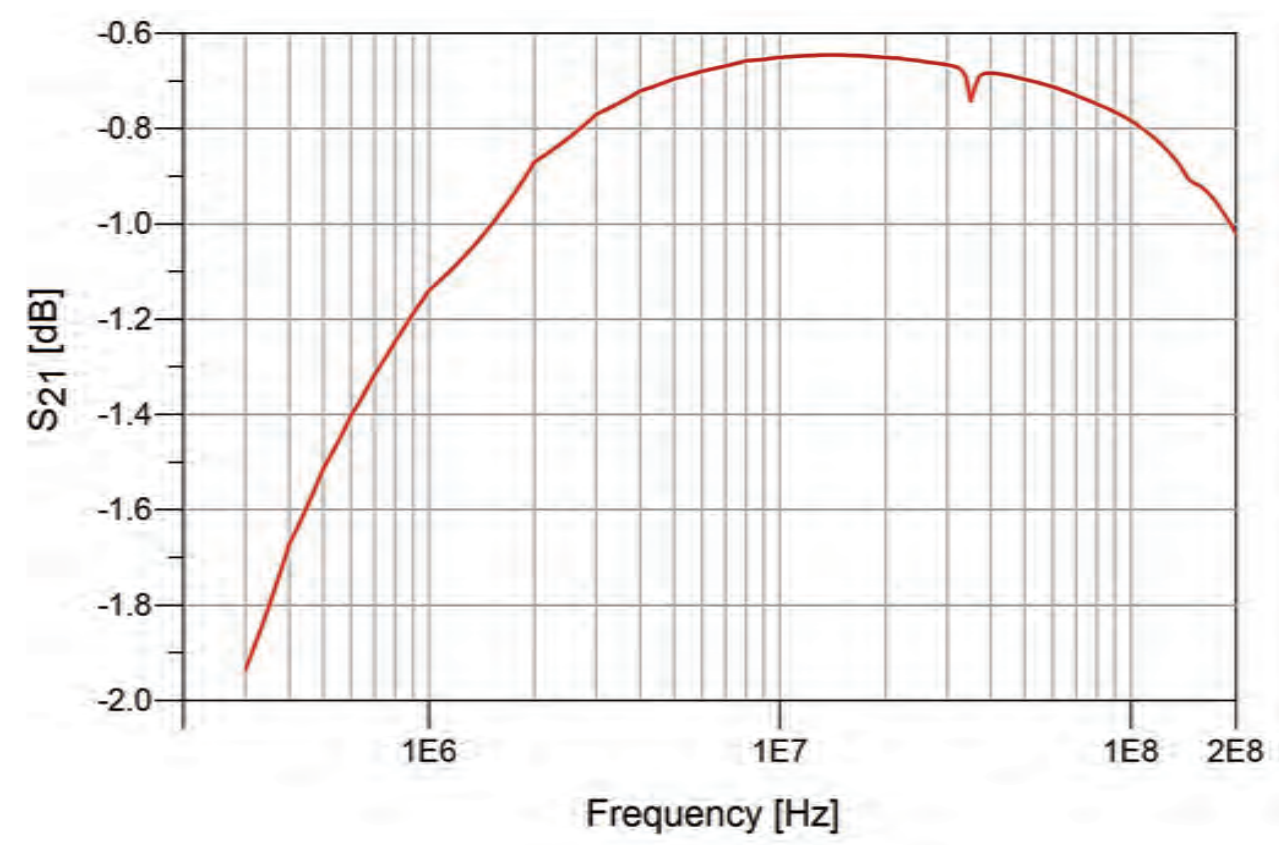

Figure 3.8: The balun loss at desired frequency range of this study. 
wires directly were connected from MiniVNA Pro to the on-body transmitter and receiver electrodes, whereas four on-body electrodes setup (figure 3.9) were used to examine the measurement setup with baluns. Results show that the IBC measurement without baluns has 3 to $55 \mathrm{~dB}$ less loss compared to the setup with baluns, below 80 MHz. This could be explained by the presence of common ground in the measurement setup without baluns. To provide the real measurements set up for IBC, the MiniVNA

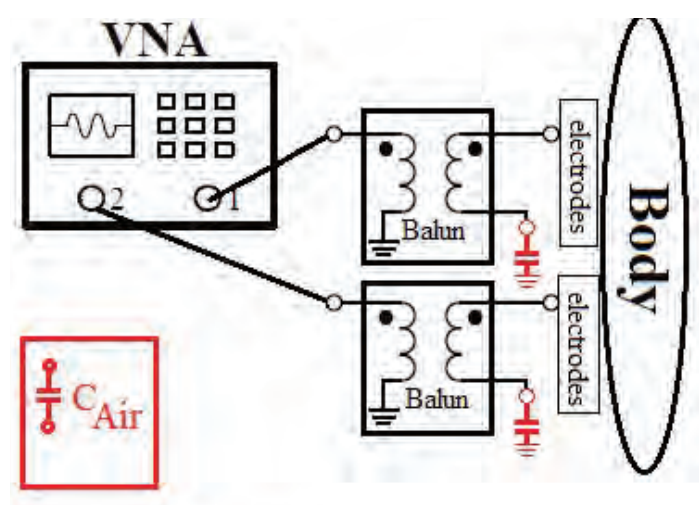

(a)

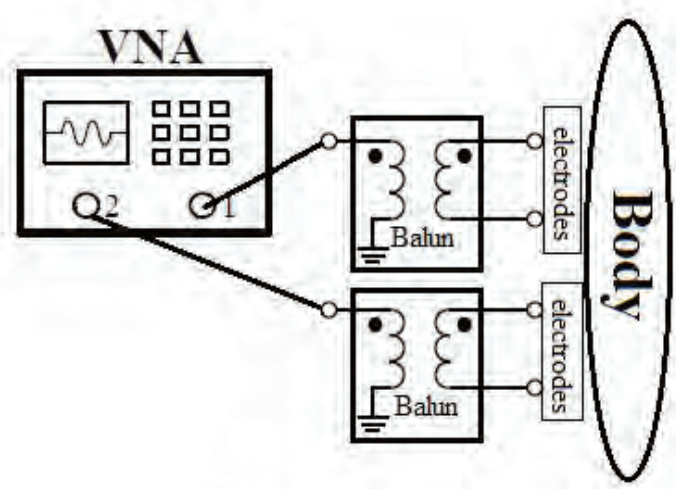

(b)

Figure 3.9: The IBC measurement setup: (a) two on-body electrodes and (b) four on-body electrodes configurations 


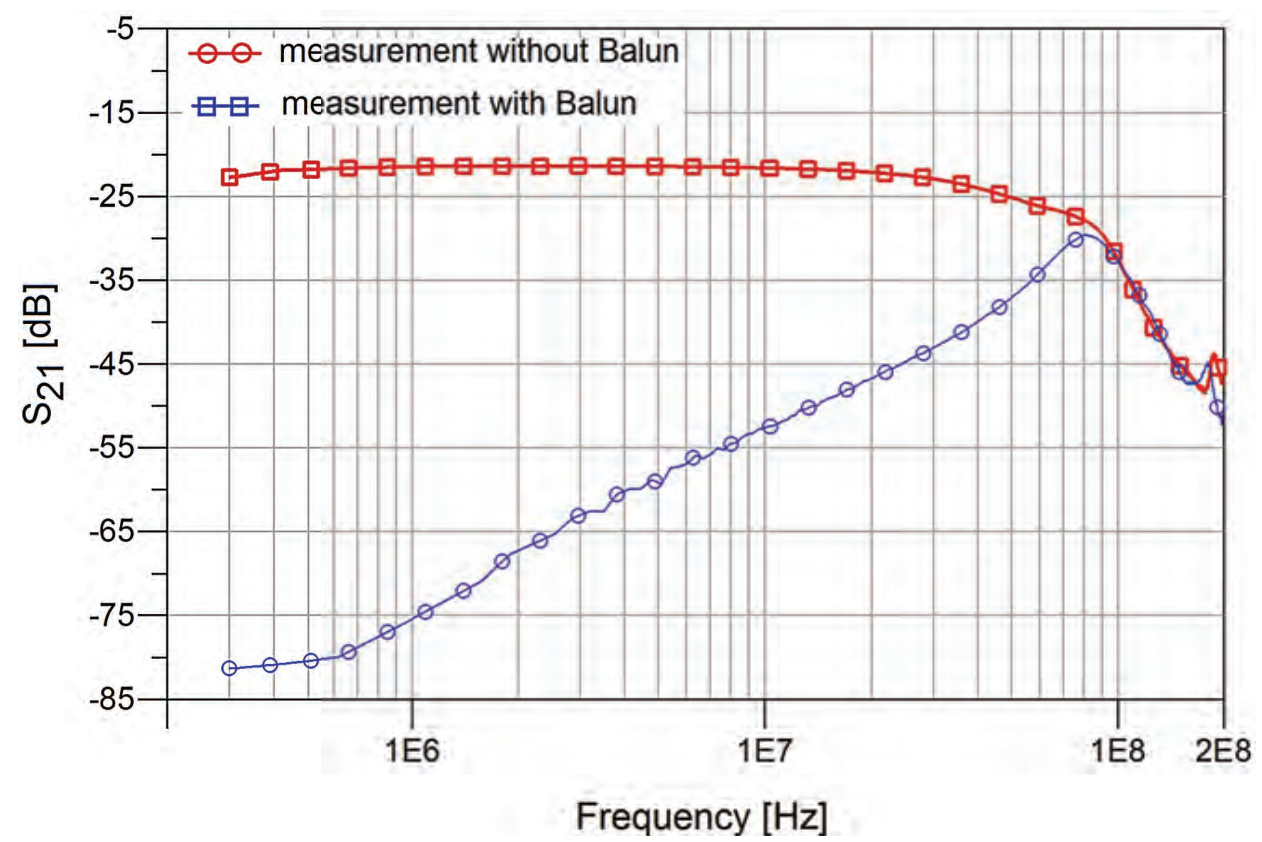

Figure 3.10: The variation of $S_{21}$ due to the presence of balun in the IBC measurement setup.

Pro ports need to be decoupled from the common point by means of baluns. Results show the similar behavior for the $S_{21}$ of with and without balun, above $80 \mathrm{MHz}$. This indicates that the signal is propagated through the environment rather than through the body.

Identifying a certain frequency range is necessary for IBC since at higher frequency the signal wavelength $(\lambda)$ becomes comparable to body height and the electromagnetic radiation will be generated through the air [35]. It is worth noting that the body height refers to the electrical dimension of the body rather than physical one [78]. The appropriate choice of operation frequency range (bandwidth) therefore, contributes significantly to data transmission rate through the body as a communication channel medium. Meanwhile, we could not ignore the critical role of wires $(115 \mathrm{~cm}$ long) in delivering the signal to the receiver. Hence, the preliminary experiments in this study 

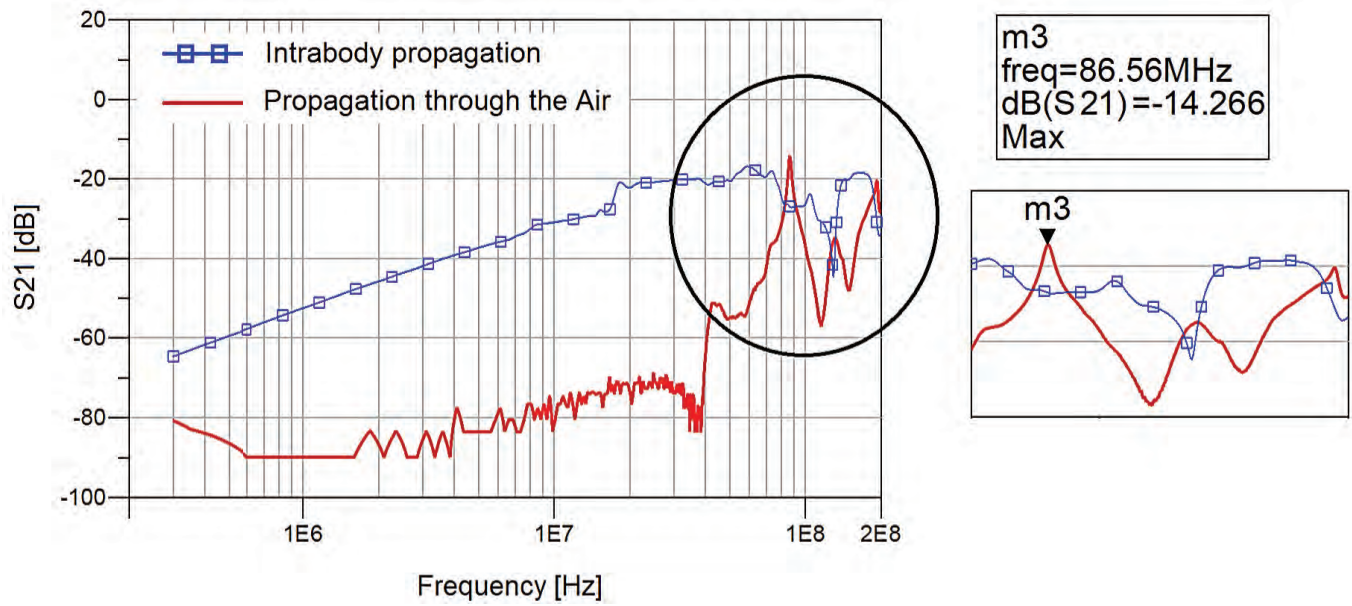

Figure 3.11: Comparison of signal attenuation through the human body and air.

were carried out with two main purposes: first, to identify the suitable frequency range where there is no signal radiation through the air; second, to examine the possible effects of wires, which could operate as an antenna, on signal delivery. In this sense, we considered the human body and the air as two separate transmission paths, to examine the process of signal propagation. The measurement was firstly carried out without connecting any electrodes to the body (propagation through the air). Then in the second step, electrodes were attached to the body forearm (intrabody propagation). It should be noted that both measurements were carried out in the same location (electronics laboratory). Additionally, the channel length between the transmitter and receiver electrodes was fixed to $20 \mathrm{~cm}$. Figure 3.11 indicates the comparison of both the human body and air channel loss. Below $80 \mathrm{MHz}$, up to $25 \mathrm{~dB}$ less loss is observed when signal is propagated through the body channel compared to when it is transmitted through the air surrounding the body. This could be due to the high conductivity of the body tissues (skin conductivity is $0.415 \mathrm{~S} / \mathrm{m}$ at 54 $\mathrm{MHz}$ ) [1]. Above $80 \mathrm{MHz}$, however, the achieved results from communication through 


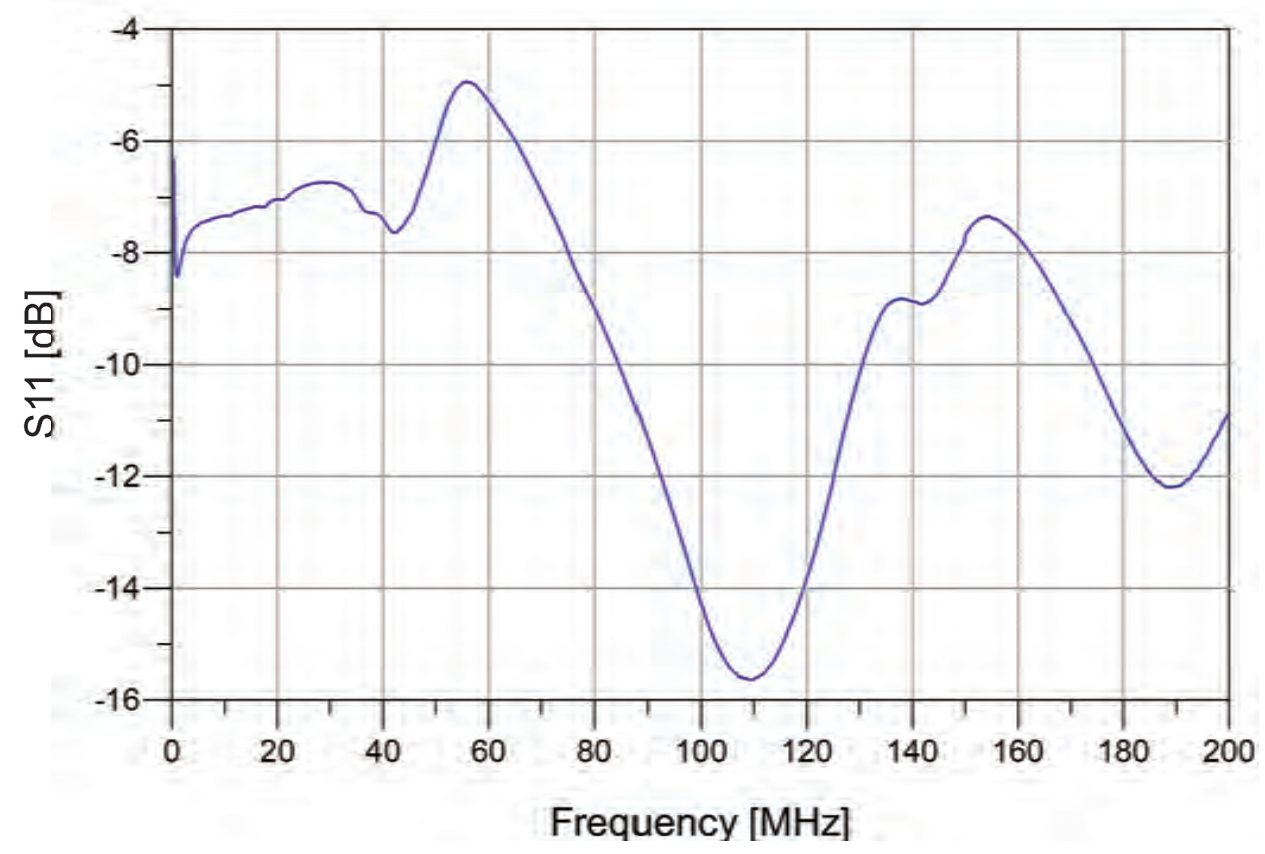

Figure 3.12: The measured body channel reflection coefficient $\left(S_{11}\right)$ by means of miniVNA Pro.

the air reveals less attenuation $(7.0 \mathrm{~dB}$ at $86.5 \mathrm{MHz})$, in some points, compared to the condition involving body limb. At these frequencies, since the relative permittivity of body tissues are less than lower frequencies (figure 2.4), the electrical body size becomes comparable with the wavelength. Hence, the antenna effects of the human body as well as the connecting wires need to be taken into account above $80 \mathrm{MHz}$ frequency band [78].

Figure 3.12 shows the reflection coefficient of the body channel $\left(S_{11}\right)$ while only transmitter electrodes were attached to the upper arm. The achieved results indicate that the body channel reflection coefficient is less than $10 \mathrm{~dB}$ below $82 \mathrm{MHz}$. Since this value is increased up to $16 \mathrm{~dB}$ in the frequency range between $82 \mathrm{MHz}$ to 130 $\mathrm{MHz}$, the frequency range below $82 \mathrm{MHz}$ with lower reflection could be suitable for the IBC technique. 


\subsection{Summary}

In this chapter, the employed measurement system for two IBC structures was presented. The utilization of battery-powered miniVNA provided us with the opportunity to perform out door measurement, to carry out experiments on dynamic body postures, and to decouple the measurement equipment from $50 \mathrm{~Hz}$ main power line (for further safety of IBC devices). Also, the components of IBC measurement setup (electrodes, baluns, and cables) were further elaborated so as to have a detailed understanding of the process of IBC technique. The rationale behind equipment preferences was discussed. For instance, the utilization of the balun, a distinguished characteristic of our measurement setup, aimed to prevent the parasitic return path from being shorted to the common voltage of the miniVNA battery in capacitive coupling IBC. Finally, the advantages and limitations of each element were discussed in this chapter. 


\section{Chapter 4}

\section{Empirical Measurement}

The mobility of the human body should be considered in BAN applications, particularly when IBC is employed for data communication. It is important to understand the dynamic body channel characteristics, as they affect the optimal operation frequency range and thus the data transfer rate for IBC. Therefore, in-vivo measurements are required to understand the body channel characteristics and constraints so as to propose new channel models for human body transmission medium. Many research projects are currently focusing on improving the performance of static IBC systems. Although dynamic motion studies for IBC have shown that the complex body movement such as walking [27], affects data transmission, no previous study has qualified the changes in signal loss on different frequency bands during smaller segment movements such as limb joints flexion and extension. Further studies are required to investigate the body posture effect, the influence of body dynamics, and the effect of surrounding environment on IBC.

The continuous body movements in daily activities are effectuated by joint-segments motions. This study aims to investigate details of body motion on IBC considering 
limb joint effects rather than complex body movements. Successful completion of this measurement on real human body will significantly contribute to enhancing the previous IBC models by taking the body limbs effects (such as joint) into account. More precisely, since body movements are mainly permitted by joint-segments and joint position monitoring is essential for posture analysis such as gait, the effect of joints in the signal path during IBC is investigated in the present chapter. This chapter is organized under five section headings: Section 4.1 presents the influence of joint-segments on IBC. The body movement effects on IBC are examined through the limb joints motion in both upper and lower human limbs in section 4.2. The influence of distance between transmitter and receiver electrodes through different joint angles is discussed in section 4.3. In section 4.4, we study the influence of various measurement locations and environments on signal transmission during IBC, both indoor and outdoor. Finally, section 4.5 provides a summary of this chapter and highlights the significance of the achieved results. It also explains how the obtained results will contribute to understanding of the IBC system in the future.

\subsection{Influence of Joint-Segments}

Body movements are mainly permitted by joints in a hard and rigid skeleton [30]. They are a linkage between two or more bones and their anatomy is slightly different from limb segments (see figure $4.1[77]$ ). Joints are protected by cartilage which is a special connective tissue between bones and have smaller amount of muscle compared to other parts of human body. Tendons of several muscles cross the joint and provide secure human body motions [30]. Therefore, due to different tissue layers of joints compared to the segments of the body without joints, the presence of joints in a 


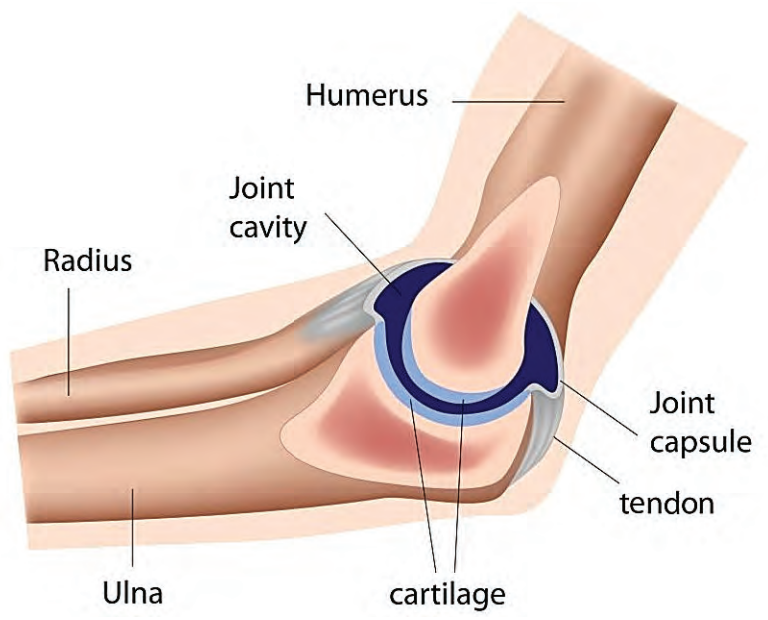

Figure 4.1: Elbow joint anatomy [www.iphysioperth.com.au].

human communication pathway has effect on signal propagation. Understanding the dielectric properties of joint layers will be helpful in further analysis of the signal propagation, reflection, and attenuation.

IBC is classified into two basic methods in terms of the positions of on-body electrodes which are used in transmitter and receiver sides (see figure 3.9). In the first method, only one of the electrodes is attached to the body in each side and the other electrode is floated between the body and external ground. Therefore, this method is referred to a "two-electrode configuration". The IBC signal return path is created through the capacitance between the floating electrodes and earth ground in this method. Since the capacitance is a function of the distance between the floating electrodes and earth ground, the variation of the distance during body movements affects the IBC signal propagation. The second method is the "four-electrode configuration", in which a pair of electrodes is attached to the body in both transmitter and receiver sides. The signal propagation in this method is confined to the body tissues particularly below $5 \mathrm{MHz}$ [57]; hence, the body tissue compositions have considerable 
effects on IBC using this method. We expect the higher signal attenuation from the "four-electrode configuration" compared to the "two-electrode configuration" method, specifically during the presence of joints within the body channel below $5 \mathrm{MHz}$. Due to the reduction of tissue penetration depth at higher frequencies, using this method could strength the influence of external ground rather than the tissue composition. Generally, the influence of earth ground becomes inevitable in both IBC methods and during the body movements. In this study, the on-body measurement points are divided to upper and lower limb based on the farthest and the closest proximity to the external ground, respectively. Since the arm and leg are commonly used as monitoring points in biomedical applications such as blood pressure monitoring, the empirical measurements are concentrated around the elbow and knee joints as representatives for other body joints. The influence of joint-segments within the IBC channel is studied through measuring the signal attenuation. We present empirical results to demonstrate the effect of joints on signal transmission and channel attenuation.

Protocol: In this part of study, three male subjects (30-33 years old) volunteered to participate. The subjects were $178-182 \mathrm{~cm}$ in height and $65-78 \mathrm{~kg}$ in weight. To measure the signal attenuation the MiniVNA Pro was used (see more details in chapter 3). The MiniVNA Pro was calibrated (using standard open, short, and load calibration kits) to drive AC current to the human body below $1.0 \mathrm{~mA}$ which is 20 times below the maximum allowed contact current. The maximum signal transmission power according to ICNIRP exposure guidelines [84] is $37 \mathrm{dBm}(=5 \mathrm{~W})$ for an average weight of $65 \mathrm{~kg}$. The source power from the MiniVNA Pro was fixed at a lower input power of just $0 \mathrm{dBm}(=1.0 \mathrm{~mW})$ for further safety. The sweep signal frequency range was $0.3-200 \mathrm{MHz}$ with a $50-\Omega$ impedance on the MiniVNA ports. Preparation protocol and setup follows that detailed in chapter 3 . 


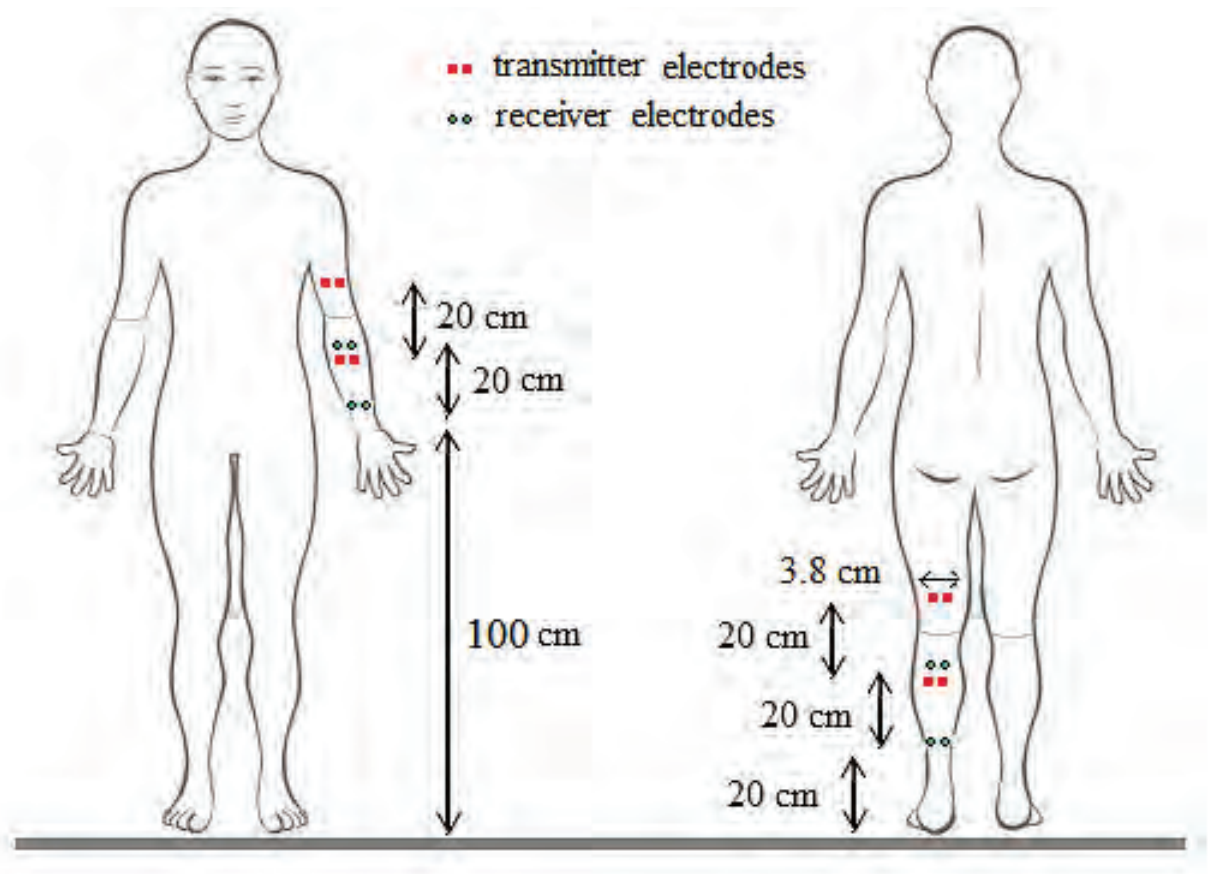

Figure 4.2: Transmitter and receiver electrodes points over the human body.

In all experiments, the subject was asked to stand in a relaxed manner. The transmitter and receiver electrodes were attached on the subject's left side (left arm and leg) while the inter-electrode distance was $3.8 \mathrm{~cm}$. Figure 4.2 shows the transmitter and receiver electrodes points on the subject's body. Receiver electrodes are placed on the forearm and calf while the transmitter electrodes were positioned on the upper arm, forearm, thigh, and calf. Since this study sought to investigate limb joints effects on IBC, the presence of limb joints through the communication path (between transmitter and receiver) was necessary. The achieved results from the IBC channel with joints need to be compared with no joint positions to confirm the influence of joints on signal transmission. The channel length for both positions was considered equal (20 $\mathrm{cm}$ ). The distance was deliberately set to be no longer than $20 \mathrm{~cm}$ for the following reasons. First, possible environmental interference is minimized in shorter channel 


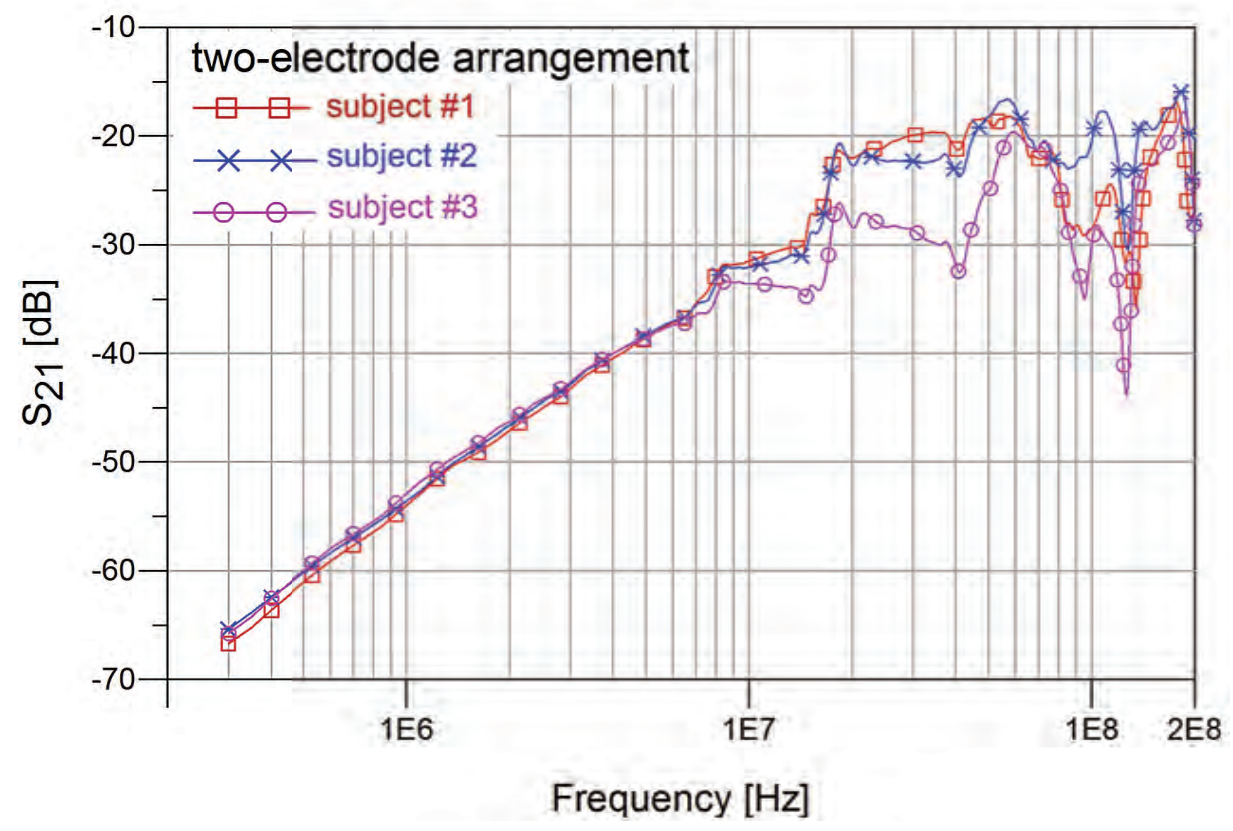

Figure 4.3: Signal attenuation of all subjects during the two-electrode configuration and the presence of elbow joint within the channel.

length. Second, the $20 \mathrm{~cm}$ is a short enough distance between the transmitter and receiver to involve the limb joint. The transmitter and receiver electrodes had equal distance $(10 \mathrm{~cm})$ from either elbow or knee joint. For the two-electrode arrangement, the floating electrodes of the transmitter and receiver were perpendicularly fixed at a distance of $5.0 \mathrm{~cm}$ from the body. The measurements for each position were repeated three times and the average attenuation was recorded for each test subject.

\subsubsection{Two-electrode Configuration}

The overall achieved results of this study demonstrated that the presence of limb joints across the signal transmission path increases channel loss in IBC. Figure 4.3 shows the effect of elbow joint on signal attenuation during two-electrode configuration for all three subjects. We have calculated the average and standard deviation values between 

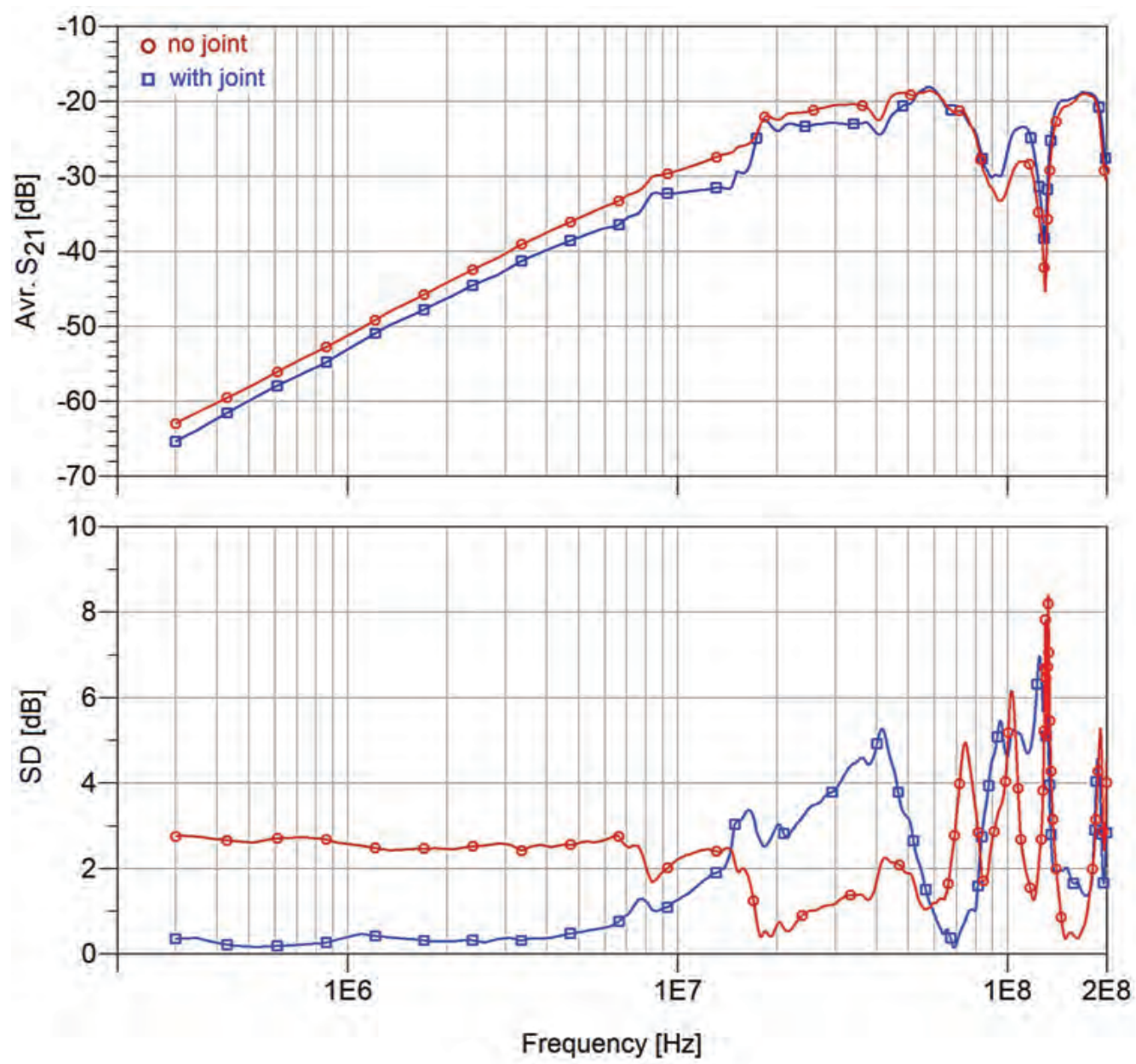

Figure 4.4: The average (Avr) and standard deviations (SD) of attenuation between three subjects. Results show the effect of elbow joint along the signal pathway during two-electrode IBC configuration.

the subjects. Figure 4.4 compares the average (Avr) and standard deviations (SD) of the signal attenuations during joint and no joint positions. The signal attenuations in the presence of elbow joint is $2.5 \mathrm{~dB}$ higher than those obtained without joints at the frequencies below $10 \mathrm{MHz}$. However, the attenuation difference reaches 5.62 $\mathrm{dB}$ at frequency range $10-20 \mathrm{MHz}$ which is the center frequency region for the IEEE 802.15.6 IBC standard [17]. 
At the frequency band of 20-60 MHz, the body channel with joint shows higher attenuation (less than $3.0 \mathrm{~dB}$ ) compared to that without joint in the signal path. Finally, the measurement results above $60 \mathrm{MHz}$ show that, the presence of joints has negligible effect on signal propagation path and the attenuation behavior. Further, standard deviation between all subjects varies from less than 1 dB below $10 \mathrm{MHz}$ to $4.5 \mathrm{~dB}$ at $42 \mathrm{MHz}$ in presence of joint. That is, the body channel with different tissue compositions and joints led to the maximum deviation of $4.5 \mathrm{~dB}$ at the IBC IEEE 802.15.6 frequency band [17]. The results from all subjects indicate less than $2.5 \mathrm{~dB}$ standard deviations in the channels without joint at the same frequency region. Therefore, the variation of communication mediums (i.e., different test subjects) affects the IBC to a lesser extent during forearm experiments.

Figure 4.5 shows the knee joint effect on signal attenuation during two-electrode configurations. This figure plots the average value and standard deviation of attenuation between all three subjects. Below $10 \mathrm{MHz}$, the channel with the knee joint indicates higher attenuation (less than $1.0 \mathrm{~dB}$ ) compared to that without joint in the signal path. At frequency range 10-60 MHz, the difference increases to approximately $3.0 \mathrm{~dB}$. However, above $60 \mathrm{MHz}$, the presence of joint presents a negligible influence on the signal attenuation. Figure 4.5 also indicates that the difference of standard deviations for both positions is less than $0.5 \mathrm{~dB}$ within the desired frequency range of this study. Therefore, the effects of the knee joint in different test subjects on IBC are negligible during the signal transmission in the lower limb. This could be attributed to the influence of the external earth ground which is more dominant in the two-electrode configuration [28]. 

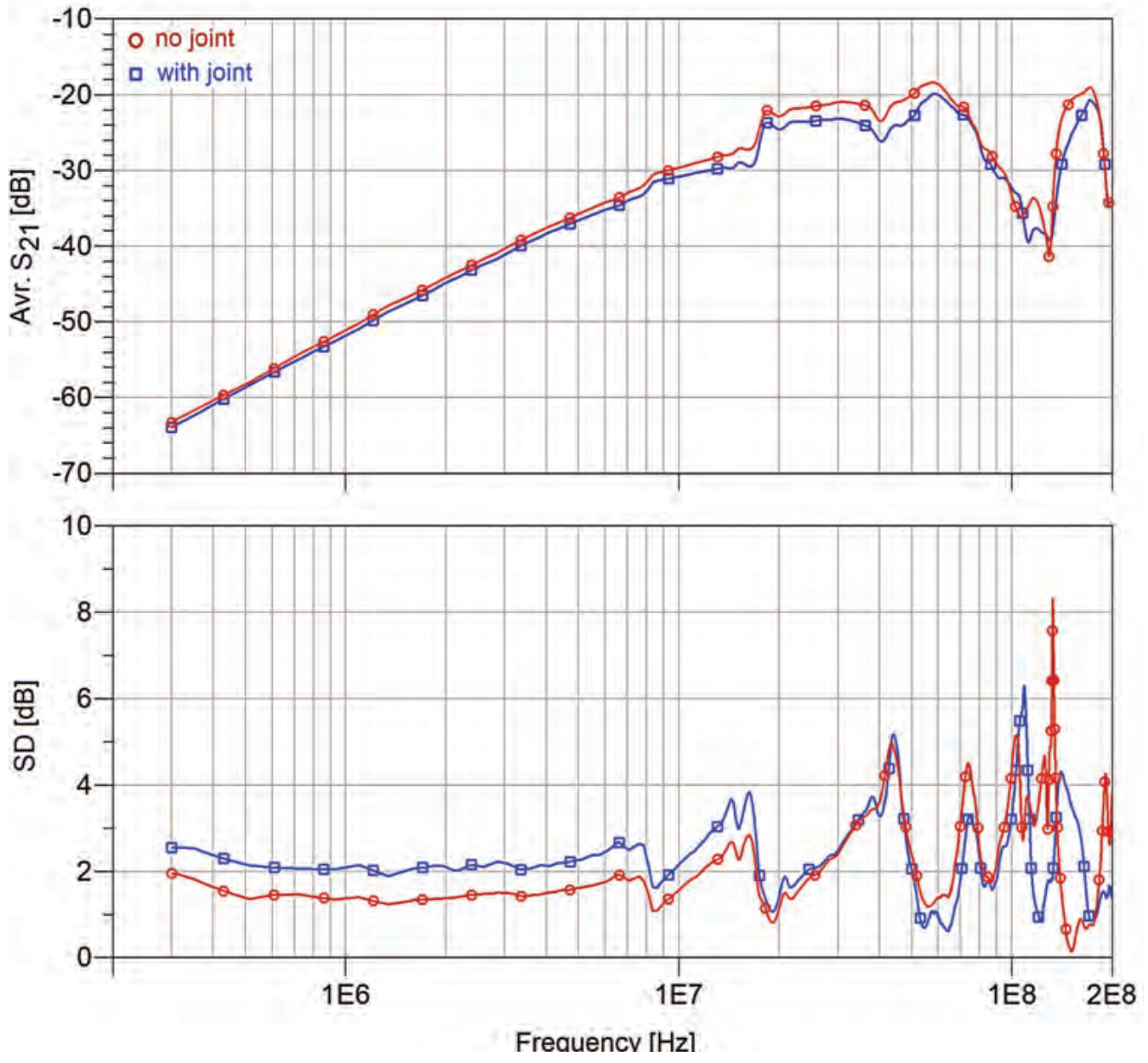

Figure 4.5: The measured attenuation average (Avr) and standard deviation (SD) between all subjects. The knee joint effect on the IBC signal propagation is demonstrated for two-electrode configuration.

\subsubsection{Four-electrode Configuration}

Figure 4.6 shows the results from three subjects in presence of elbow joint and during four-electrode configuration. The influence of elbow joint on signal attenuation is compared with no joint position in figure 4.7. The average and standard deviation are calculated between the achieved results from three subjects (see figure 4.6). The results indicate that the elbow joint causes up to $6.5 \mathrm{~dB}$ higher attenuation, below 20 


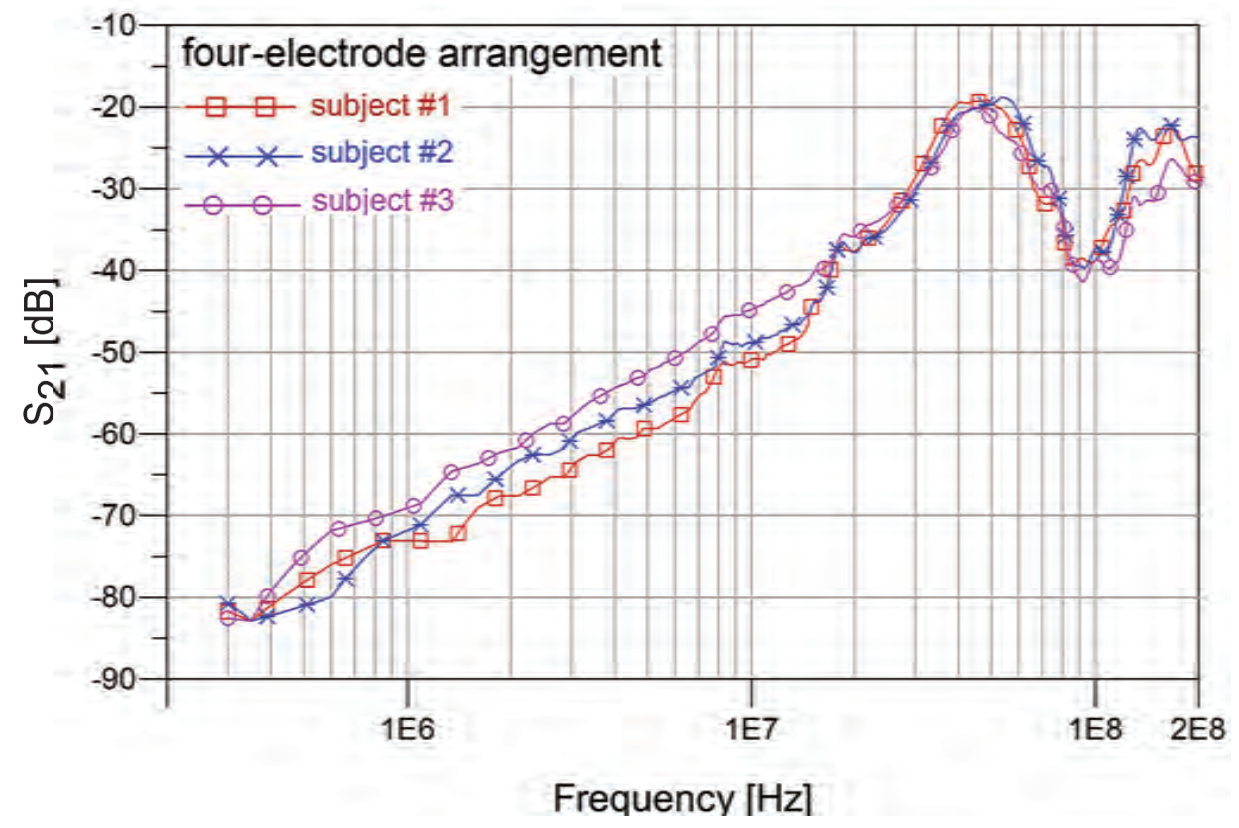

Figure 4.6: The influence of elbow joint on the signal propagation during fourelectrode configurations.

MHz. This value varies from $6.52 \mathrm{~dB}$ at $0.3 \mathrm{MHz}$ to $3.0 \mathrm{~dB}$ at $5.0 \mathrm{MHz}$ and reaches to $1.12 \mathrm{~dB}$ at $15.0 \mathrm{MHz}$ (see Figure 4.7). Above $20 \mathrm{MHz}$, the presence of the elbow joint within the transmission path has negligible effects (less than $1.0 \mathrm{~dB}$ loss) on signal attenuation variations. The measurement results show that the maximum standard deviation of no joint position is up to $2.0 \mathrm{~dB}$, below $20 \mathrm{MHz}$. This doubled to $4.0 \mathrm{~dB}$ when a joint was present in the transmission path. This could be due to the various elbow joint sizes of individual subjects with different body bone masses which varies based on several factors such as age, genetics and so on [76].

Figure 4.8 shows the knee joint effects on the signal transmission through the human body while four on-body electrodes were employed in the transmitter and receiver sides. The average results from three test subjects indicate that the signal propagation at frequencies below $60 \mathrm{MHz}$ cause less than $3.0 \mathrm{~dB}$ higher attenuation 


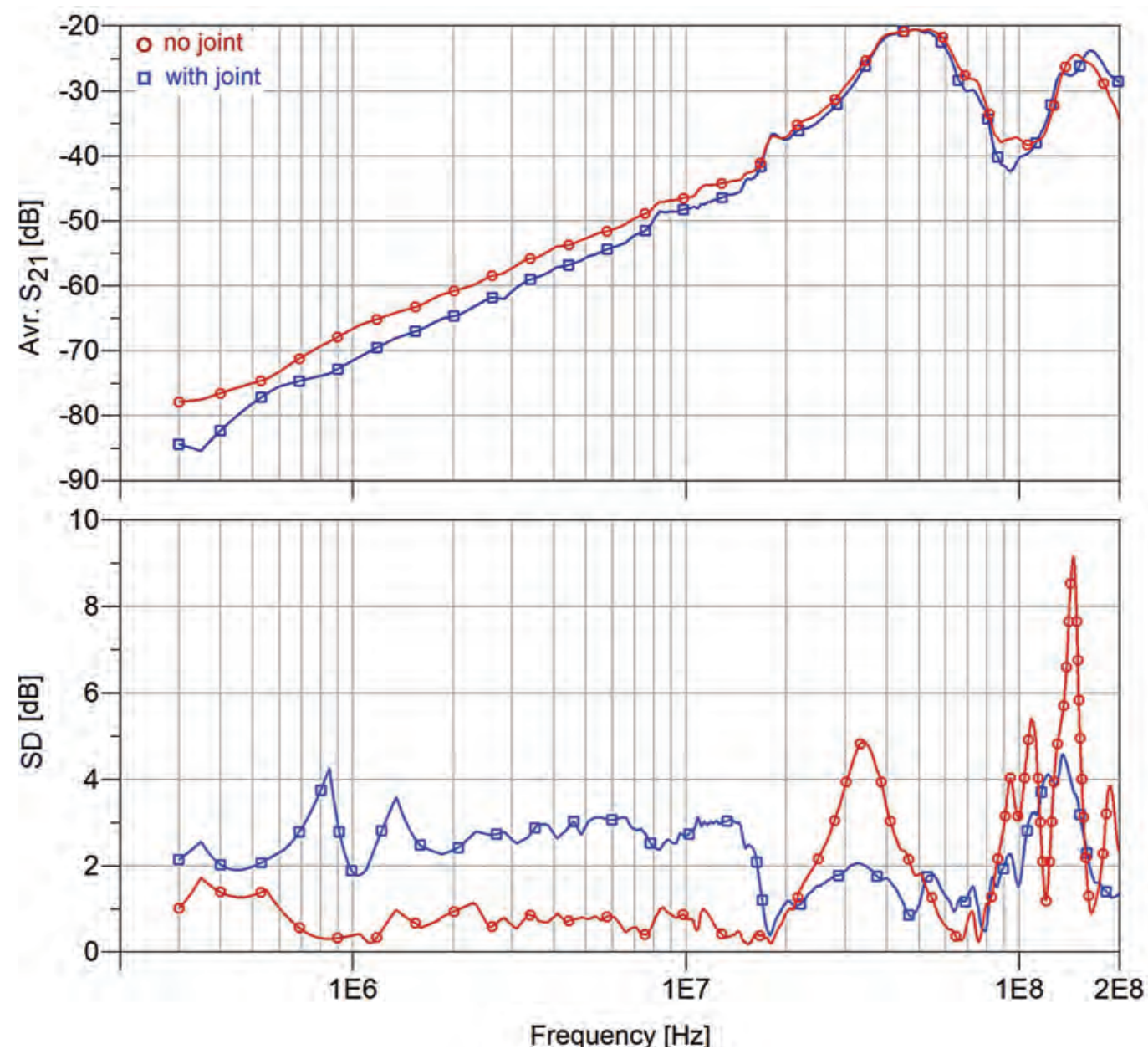

Figure 4.7: The influence of elbow joint on signal attenuation during IBC with fourelectrode configurations. The average (Avr) and standard deviation (SD) are calculated between the achieved results from all subjects.

due to knee joint effect. Above $60 \mathrm{MHz}$, the influence of the knee joint is insignificant enough (less than $1.0 \mathrm{~dB}$ ) to be ignored. The standard deviations of the achieved results indicate that the behavior of propagated signal is approximately less susceptible to various subjects with different knee joints, above $1.0 \mathrm{MHz}$ frequency. The maximum standard deviation is $4.6 \mathrm{~dB}$ which occurs around $40 \mathrm{MHz}$ for both channel conditions, knee joint and no knee joint. The standard deviation indicates that IBC 


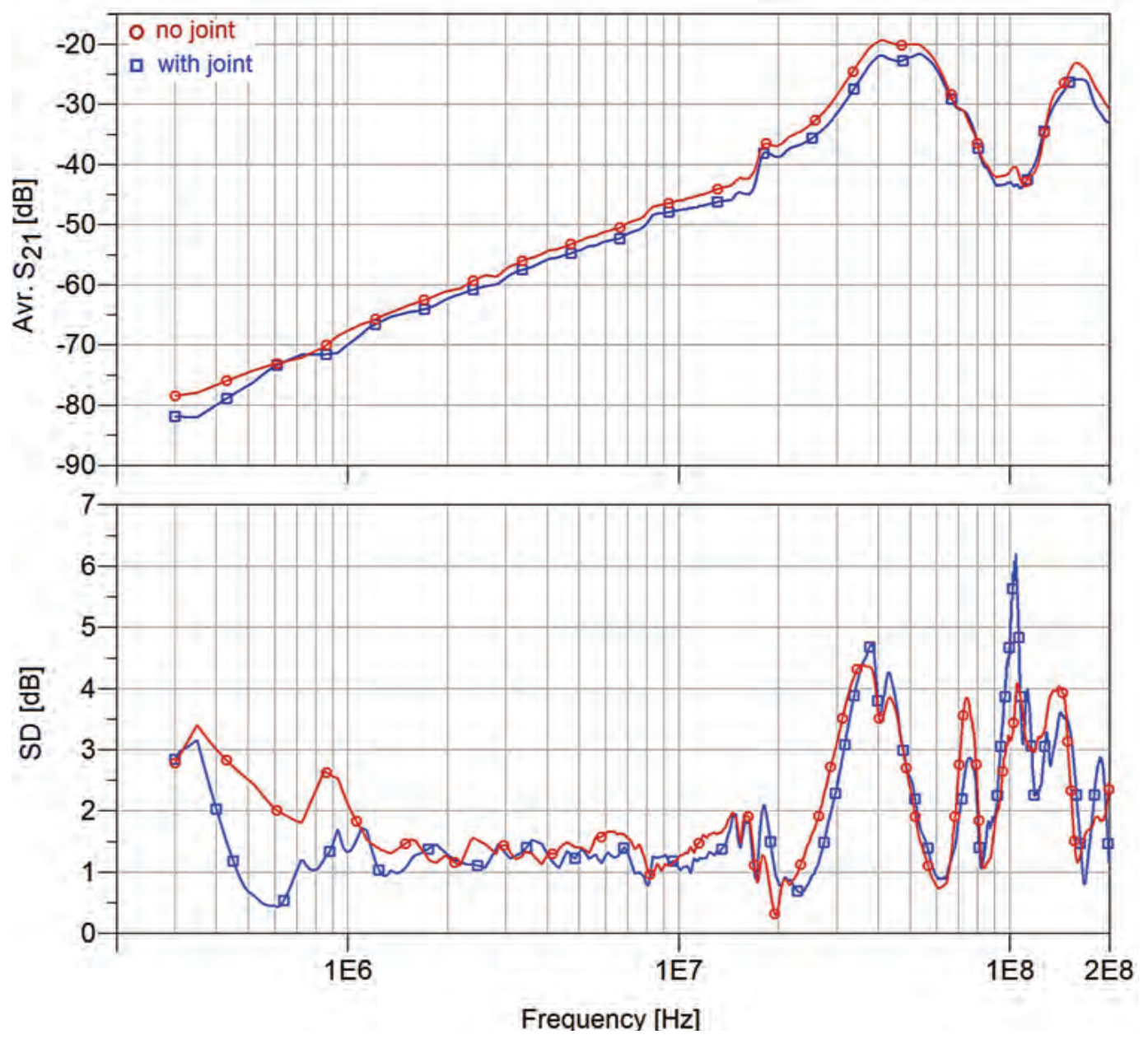

Figure 4.8: The knee joint effects on signal attenuation during four-electrode configurations. The calculated average (Avr) and standard deviation (SD) are plotted for both channels, with and without joints.

system with four-electrode configuration is susceptible to the variation of channel (i.e., different subjects), above $40 \mathrm{MHz}$.

\subsubsection{Discussion}

According to Wegmueller et al. [72], the signal attenuation on arms and legs exponentially increases with the distance between on-body transmitter and receiver. 
However, this could not only be attributed to the communication channel length increase. The achieved results in this chapter indicate that the signal transmission through two equal-distance communication channels i.e., channel with joint and channel without joint, result in different signal attenuation. Our results demonstrated that three main factors contribute to the variation of the signal attenuation for a constant channel length. First, the frequency range which the signal occupies for communication through the human body. To determine the optimal frequency range, a wide frequency range up to $200 \mathrm{MHz}$ has been chosen in this study. Second, the type of joint located along the communication path. We investigated the effects of the elbow and knee joints by performing measurements on the subjects' arm and leg. Third, the arrangement of electrodes attached on the human body (two- or four-electrode configurations).

Based on the measurement results, the signal attenuation shows the same trend for two- and four-electrode configurations within the desired frequency range of this study (0.3-200 MHz). We can divide the attenuation curves into two frequency regions; 0.3 to $60 \mathrm{MHz}$ and $60-200 \mathrm{MHz}$. In the first region, the limb joint effects on signal attenuation were observed for all subjects and minimum signal attenuation was achieved in this frequency band. In the two-electrode arrangement, the minimum attenuation was observed around 57.4 MHz. At this frequency, the presence of a joint along the channel caused 2.1 and $1.5 \mathrm{~dB}$ higher attenuation for elbow and knee joints, respectively (refer figure 4.4 and 4.5 ). On the other hand, minimum attenuation was found between 40-51 MHz with the four-electrode structure, where the presence of either the elbow or knee joints led to $2.0 \mathrm{~dB}$ higher propagation loss compared to the channels without joints. In the second area between 60 to $200 \mathrm{MHz}$, the presence of either elbow or knee joints has negligible effects on the channel loss (less than 
$1.0 \mathrm{~dB})$. It could be concluded that the signal propagation path, either in two- or four-electrode configurations in both lower and upper limbs, is primarily not through the body for signal frequencies above $60 \mathrm{MHz}$. Based on our achieved results and according to the IBC frequency in the IEEE 802.15.6 standard (centered at $21 \mathrm{MHz}$ with $\left.f_{B W}=5.25 \mathrm{MHz}\right)$, the first frequency region $(0.3-60 \mathrm{MHz})$ is the 'sweet spot' for the signal propagating using IBC. Therefore, the influence of joints among the IBC transmitter and receiver needs to be considered within this frequency band during the IBC architecture design.

We have divided the frequency band of 0.3-60 MHz into two narrower frequency regions of $0.3-10 \mathrm{MHz}$ and $10-60 \mathrm{MHz}$ so as to investigate the joint effects on IBC in more detail. At low frequencies (less than $10 \mathrm{MHz}$ ), electrical signal propagation occurs largely through body tissue due to their higher conductivity compared to the surrounding environment ( air $_{\text {conductivity }}$ is less than skin $_{\text {conductivity }}, 0<0.013[\mathrm{~S} / \mathrm{m}]$ ). The signal attenuation through different tissue layers largely depends on tissue dielectric properties. Hence, the joints and large bones (such as pelvic bone) are significant attenuators within the signal transmission path. The results from empirical measurement demonstrated that higher signal attenuation is caused by the elbow and knee joints along the communication path.

During the two-electrode configuration, the elbow and knee joints caused higher signal attenuations within $0.3-10 \mathrm{MHz}$. The shorter distance between the external ground and the floating electrodes in the lower limb results in lower impedance of the signal return path. Therefore, the influence of the knee joint within the IBC channel is less significant $(0.8 \mathrm{~dB}$ at $5 \mathrm{MHz})$ compared to the upper limb joint effects on the IBC signal propagation $(3.0 \mathrm{~dB}$ at $5 \mathrm{MHz})$.

For the four-electrode arrangement, both forward and return signals primarily 
propagate through the body, particularly at lower frequency below $10 \mathrm{MHz}$ (galvanic coupling). For instance, the knee joint as a largest and the most complex joint of the body caused $3.0 \mathrm{~dB}$ more loss compared to channel without the knee joint at $5 \mathrm{MHz}$. The inner layers of joints contain a large mass of bone, cartilage, and tendons [94] and they all have lower conductivity compared to muscle (see figure 2.4).

The increased frequency range above $10 \mathrm{MHz}$ leads to the reduction of tissue penetration depth [94]. Therefore, the signal transmission is confined to the body skin and the presence of the limb joints has lower influence on the signal propagation. For instance, the signal penetration depth of bone is $1.46 \mathrm{~m}$ at $5 \mathrm{MHz}$ which is three times less $(0.48 \mathrm{~m})$ at $40 \mathrm{MHz}$. Hence, less than $3.0 \mathrm{~dB}$ differences are caused by the joint presence during either two- or four-electrode configurations for signals above 10 $\mathrm{MHz}$.

Further, the results indicate that the effects of surrounding environment, particularly external ground, become more dominant at higher frequency. For instance, due to the dominant effect of the external ground during knee joint experiments (lower impedance between the on-body electrodes and ground compared to the body impedance), the knee joint influences become nearly ineffective at higher frequencies. Figure 4.5 and 4.8 indicate that by increasing the frequency, the presence of knee joints through the signal transmission path is more negligible $(2.5 \mathrm{~dB}$ at $40 \mathrm{MHz})$. This is while the knee is the largest joint of the body [72] and it is anticipated to have a strong effect on the IBC signal attenuation due to its larger portion of bone with lower conductivity (figure 2.4). Overall, the experiments revealed that using the IBC technique on the body lower limb is not dependent on joint effects and is strongly influenced by the external ground at the both configurations of electrodes. 


\subsection{Effect of Body Posture on IBC}

Normal activities and various postures of the human body are predicted to influence the direction of the signal route when IBC is employed. When the human body is in motion, the communication of information using wearable devices might be established through three different directions. The signal either couples across inner human tissues, over the surface of the skin, or through the air surrounding the human body. Although, an IBC system guides the signal via body tissues or over the skin, the propagation of the signal through the environment such as external ground is inevitable during body movements, particularly at higher frequencies. Therefore, the effect of movement is critical to the IBC applications since higher frequency and increased bandwidth is to be used for portable devices, particularly in biomedical applications, such as handheld electromyography (EMG). The body movement also plays an important role in the strength of the received signal. The variation of the channel length causes signal strength to change during movement. Both elbow and knee joints, which are the main focus for our research, classify as hinge joints. Hinge joints move in only one direction, namely, flexion and extension movements. Flexion of joint is a bending movement that decreases the angle of joint. The reverse of flexion is extension and occurs at the same joints. In this section, the effects of flexion and extension of the elbow and knee joints on signal attenuation during IBC are studied.

Protocol: To evaluate the limb joint motion effects on signal propagation, the same test subjects as in section 4.1 participated in this part of study. Again, the transmitter and receiver electrodes were attached to the left arm and left leg. The subjects were firstly asked to stand relaxed so as to measure the signal attenuation of the fully extended transmission path (joint angle equal to 180 degree). In the second step, we asked subjects to bend the left forearm for a joint angle of 90 degrees. As 


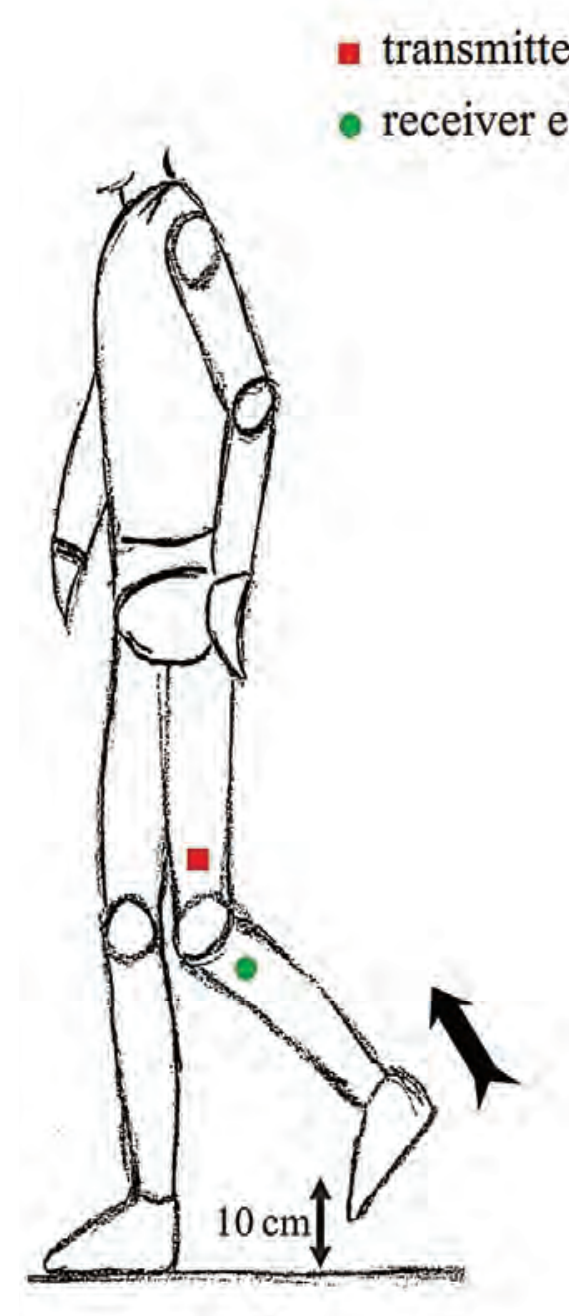

(a)

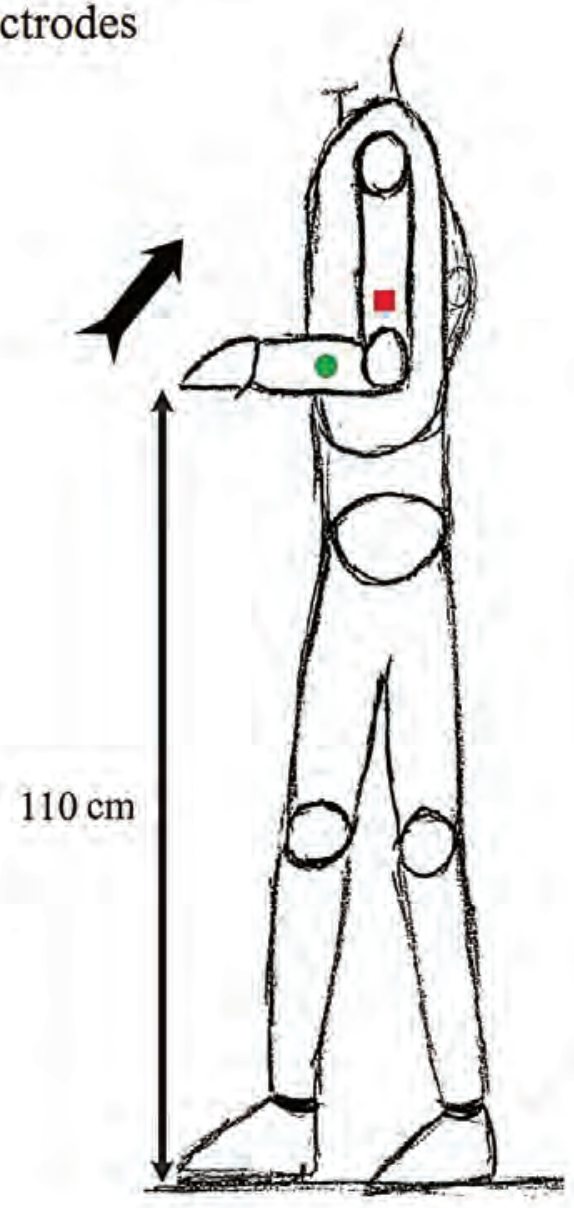

(b)

Figure 4.9: Flexion of elbow and knee joints: (a) lifting $10 \mathrm{~cm}$ toes off the floor (b) 90-degree elbow joint angle while the transmitter and electrodes are attached to the body.

shown in figure 4.9, the forearm distance from external ground was around $110 \mathrm{~cm}$. In the last position, knee joint flexion was examined while the subject was standing on the right leg with the left foot off the floor $10 \mathrm{~cm}$ apart (figure 4.9). The wooden table helped the subjects to keep their balance during the measurements on the leg. 


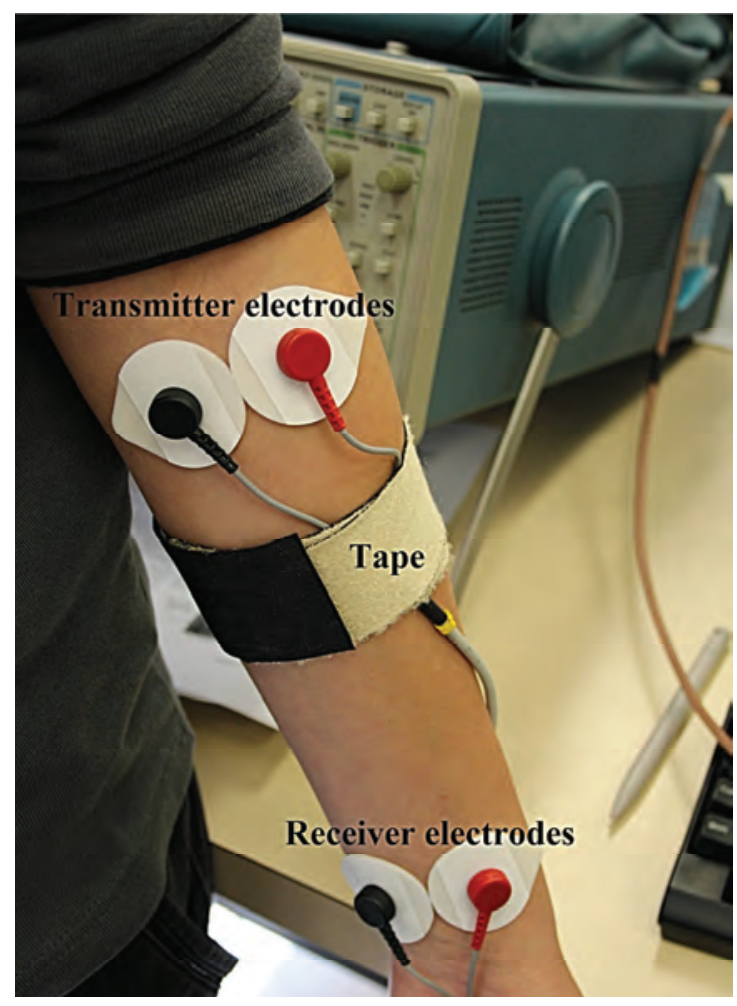

Figure 4.10: The dedicated measurement setup for joint angle motion experiments. By employing the tape, the position of connecting cables will be fixed on the body.

The connecting wires were attached to subjects' clothes using neoprene Velcro tapes (see figure 4.10) so as to minimize wire movement effects on the measurement results. The measurements were carried out three times for each subject. The average of three repeated trials for each subject was used to quantify the joints motion effects on the IBC.

Figure 4.11 and 4.12 show the measured signal attenuation using IBC for arm and leg posture effects, respectively. The attenuation curves in both elbow and knee joints flexion and extension positions could be divided to two frequency bands, $0.3-60 \mathrm{MHz}$ and above $60 \mathrm{MHz}$. We have chosen $60 \mathrm{MHz}$ because the minimum signal attenuation 


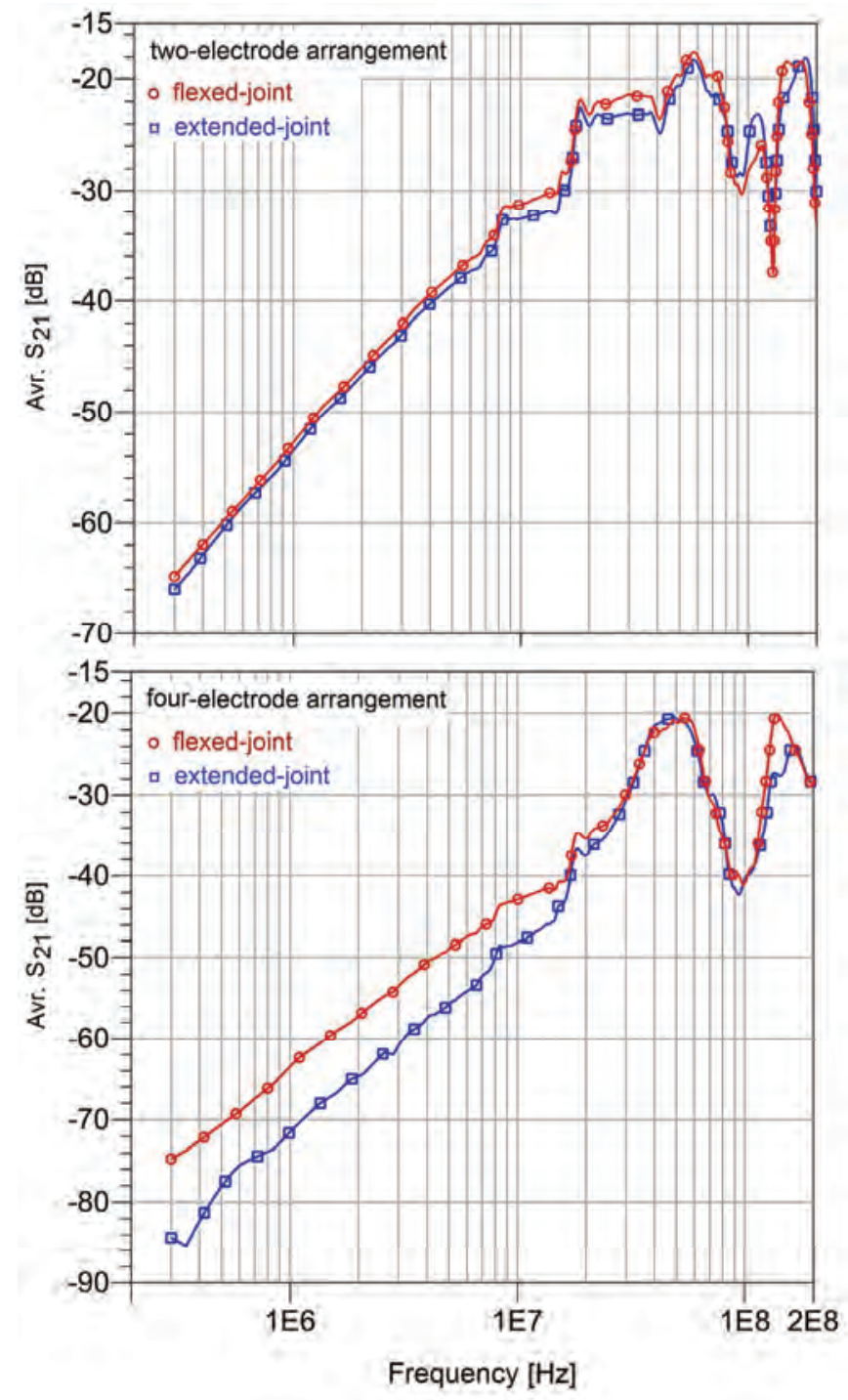

Figure 4.11: Elbow joint flexion effects on the IBC signal attenuation for both electrode configurations. Graphs show the average values of attenuations achieved from three subjects.

occurs at approximately $60 \mathrm{MHz}$ for both arm and leg motions. The measurement results indicate that at frequencies below $60 \mathrm{MHz}$, the joint flexion (smaller joint angle) leads to lower signal attenuation compared to the fully extended joint. According to the electrode configuration on the body (two- or four-configuration) as well as either 


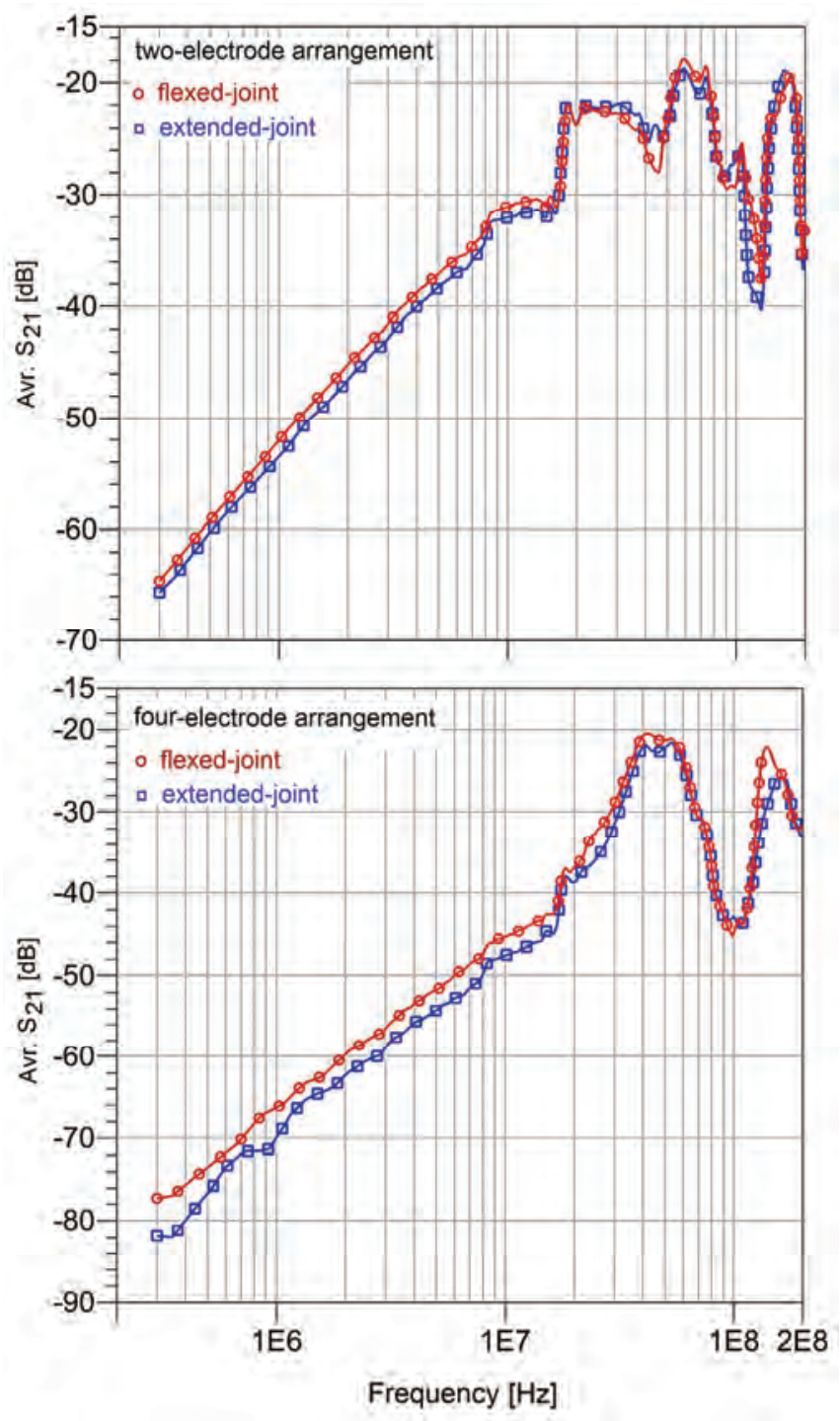

Figure 4.12: Knee joint flexion effects on signal attenuation for both IBC electrode configurations.

bending or stretching postures of the elbow and knee joints, different signal attenuations are obtained. Above $60 \mathrm{MHz}$, negligible attenuation variations are observed for both flexed limb joints during the two and four-electrode arrangements, respectively. Table 4.1 quantifies the rate of signal attenuation changes during joints motion for 
Table 4.1: The Signal Attenuation Changes During Joints Motion (Flexion) for Both Configurations of On-Body Electrodes.

\begin{tabular}{|c|c|c|c|c|}
\hline \multirow{2}{*}{$\begin{array}{c}\text { Electrodes } \\
\text { configuration } \\
\text { on the body } \\
\text { surface }\end{array}$} & \multicolumn{2}{|c|}{ Elbow } & \multicolumn{2}{|c|}{ Kneee } \\
\cline { 2 - 5 } & $\begin{array}{c}\text { Below } \\
60 \mathrm{MHz}\end{array}$ & $\begin{array}{c}\text { Above } \\
60 \mathrm{MHz}\end{array}$ & $\begin{array}{c}\text { Below } \\
60 \mathrm{MHz}\end{array}$ & $\begin{array}{c}\text { Above } \\
60 \mathrm{MHz}\end{array}$ \\
\hline \hline \multirow{3}{*}{ Two-electrode } & Less & & Less & \\
& than $1.0 \mathrm{~dB}$ & negligible & than $1.0 \mathrm{~dB}$ & negligible \\
& $@ 15 \mathrm{MHz}$ & & $\mathrm{MHz}$ & \\
\hline Four-electrode & $3.0 \mathrm{~dB}$ & \multirow{2}{*}{ negligible } & $\begin{array}{c}2.1 \mathrm{~dB} \\
\text { @1 } 15 \mathrm{MHz}\end{array}$ & negligible \\
\hline
\end{tabular}

both electrode arrangements.

\subsubsection{Discussion}

Based on empirical results, it was found that signal attenuation decreases when joint flexion occurs. As shown in figure 4.11, the maximum differences of attenuation between the flexed and fully extended elbow joint were found to occur below $60 \mathrm{MHz}$ for both two- and four-electrode arrangements, respectively. The physical proximity of the transmitter and receiver electrodes may account for the reduction of attenuation for the smaller joint angles. That is, during joint flexion, the spatial path becomes shorter than the body surface path (see Table 4.2). The signal is capacitively coupled through the air instead of flowing through the body. The distance between the receiver electrode and earth ground needs to be taken into account, particularly when the two-electrode arrangement is employed (Table 4.2). The experiments with knee joint flexion also showed decreased signal attenuation. However, the variation was less than $2.0 \mathrm{~dB}$ for both electrode arrangements (figure 4.12). The distance variation between the receiver electrodes and earth ground was very small $(1.0 \mathrm{~cm})$. It should be noted that, the four-electrode arrangement position below $10 \mathrm{MHz}$ shows between 2.0 to 
Table 4.2: Variation of Distance between Transmitter ( $\mathrm{Tx})$ and Receiver ( $\mathrm{Rx})$ Based on Joints Motion.

\begin{tabular}{|c|c|c|}
\hline \multirow{2}{*}{ Distance } & \multicolumn{2}{|c|}{ Joint Flexion } \\
\cline { 2 - 3 } & Elbow & Knee \\
\hline \hline $\begin{array}{c}\text { Between TX and RX electrodes } \\
\text { (body surface path) }\end{array}$ & $20.0 \mathrm{~cm}$ & $19.0 \mathrm{~cm}$ \\
\hline $\begin{array}{c}\text { Between TX and RX electrodes } \\
\text { (shortest spatial path) }\end{array}$ & $14.1 \mathrm{~cm}$ & From 40.0 to $41.0 \mathrm{~cm}$ \\
\hline $\begin{array}{c}\text { Between Earth and RX electrodes } \\
\text { (before and after flexion) }\end{array}$ & From 100 to $110 \mathrm{~cm}$ & \\
\hline
\end{tabular}

4.0 dB lower attenuation during knee flexion. This could be explained by the fact that the signal completely propagates through the human body (galvanic coupling IBC) below $10 \mathrm{MHz}$ and shorter spatial path has non-significant effects on signal propagation.

To examine the effects of external ground on signal propagation, both transmitter and receiver electrodes should have the similar distance to external ground (see figure 4.13). To this end, in the next section, further measurement on the human arm during two-electrode arrangement has been carried out while the arm was fully extended on the front.

\subsection{Arm Posture and Effect of Channel Length}

In this Section, we have investigated the effect of elbow joint-segment motions on signal attenuation in the two-electrode configuration of IBC.

Protocol: Three healthy subjects, two male and one female volunteered to participate in the measurements. The characteristics of chosen subjects are: 24 to 29 years old, 150 to $175 \mathrm{~cm}$ height, and 50 to $78 \mathrm{~kg}$ weight. The receiver electrodes were attached on the subject's left forearm and the transmitter electrodes was positioned 

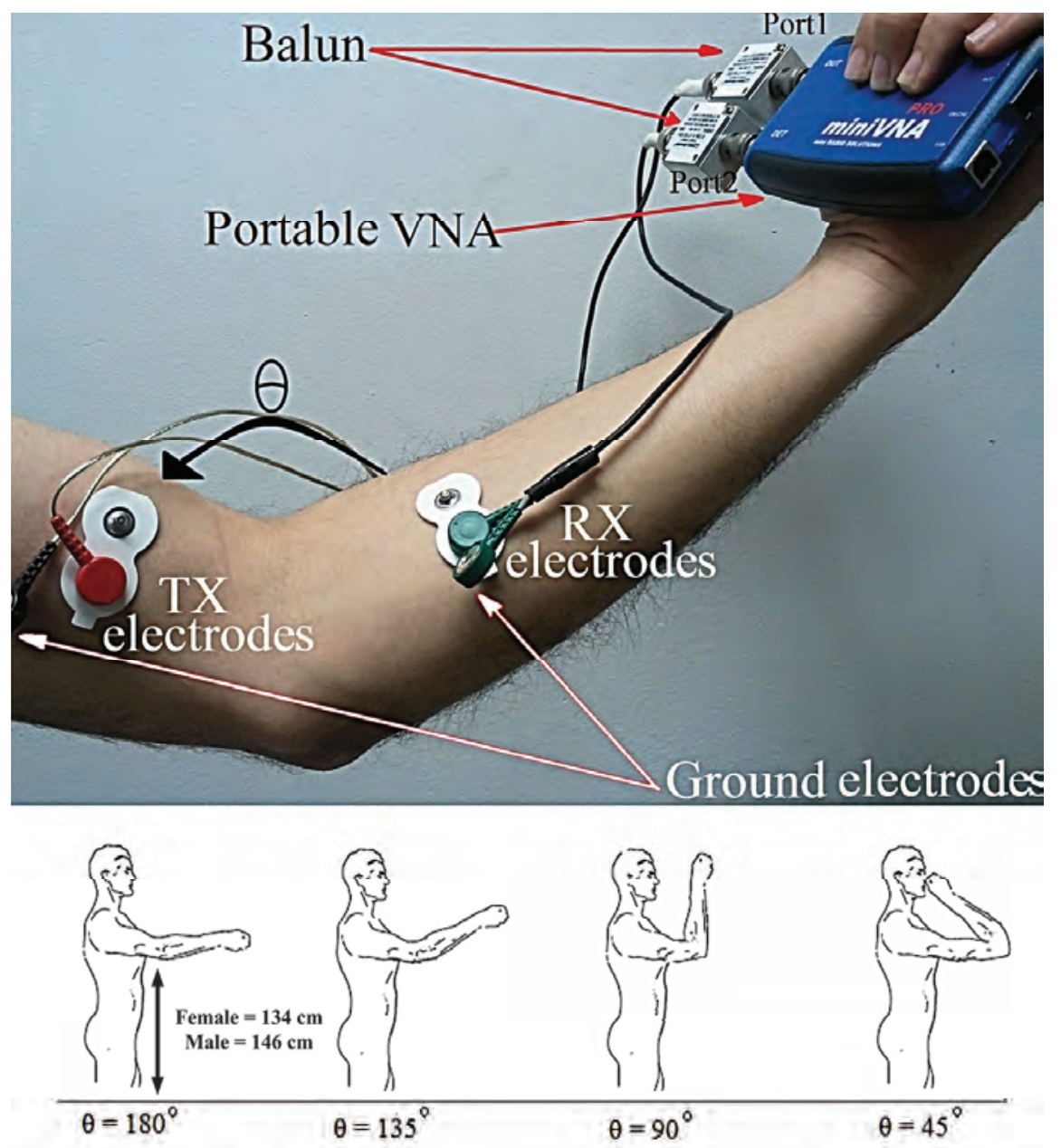

Figure 4.13: The measurement setup; $\theta$ indicates the joint angle between upper and forearm, receiver and transmitter are attached to lower and upper left arm, respectively.

on the upper left arm. Two different distances of 15 and $20 \mathrm{~cm}$ were chosen between transmitter and receiver electrodes. Figure 4.13 shows the measurement setup of elbow joint angle during IBC method. The angle between upper and forearm is $\theta$ which is varied from 45 to 180 degrees. In these measurements, the subject's was asked to stand and extend the arm out to the front. In this position, the transmitter and receiver electrodes distance from the external ground are equal during the joint 


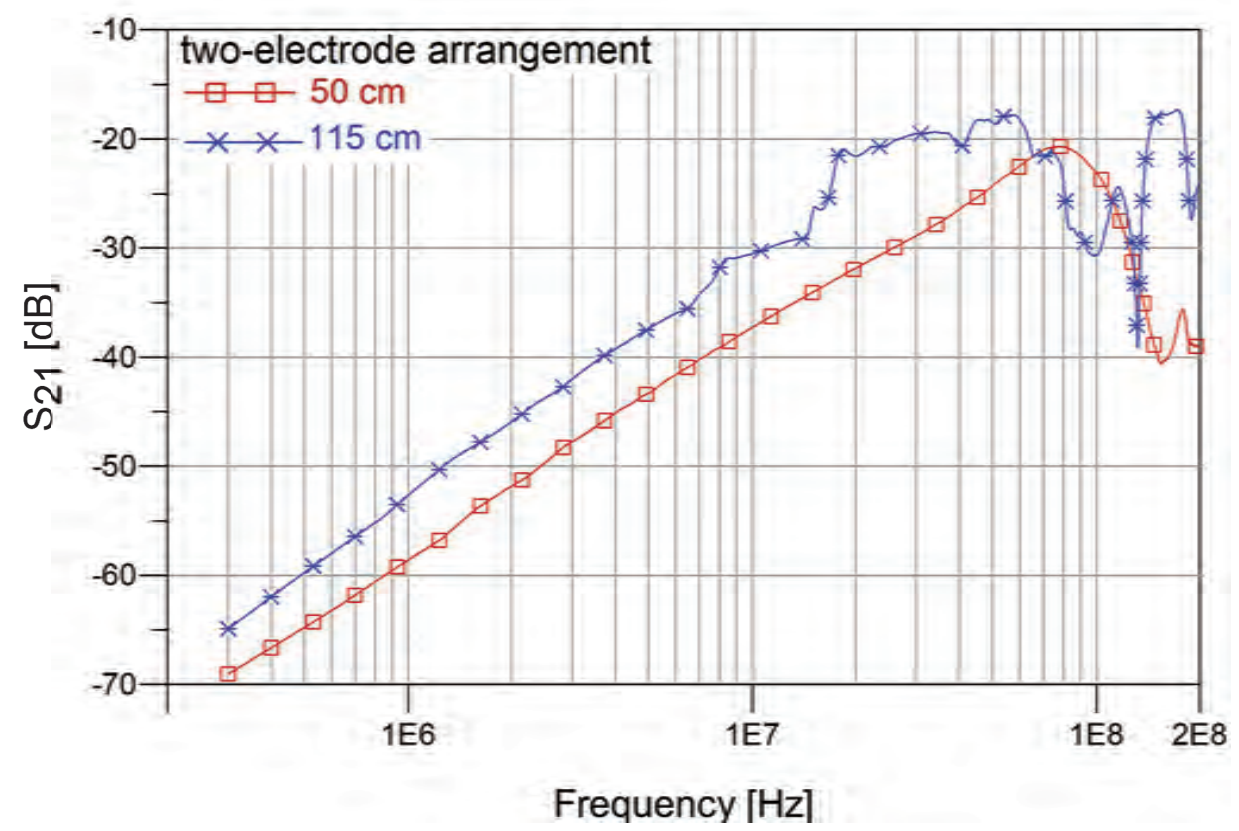

Figure 4.14: The $90^{\circ}$ flexed arm channel attenuations which measured with the two different cables.

angle of 180 degree (see figure 4.13). This distinguishes elbow joint measurements from section 4.2 measurements. In fact, the effect of external ground on the signal transmission has been decreased during our measurements in this Section. The short cables were employed between human body and baluns; hence the effect of any changes in cable positions due to different joint angle was negligible. We have compared the $50 \mathrm{~cm}$ unshielded twisted-pair cables used in this section with the $115 \mathrm{~cm}$ shielded cables which employed in two previous sections (see figure 4.14). Both cables were used to measure the attenuation of a same channel with 90 degree elbow joint angle and during two-electrode configuration. The results indicate that both cable behaviors are approximately similar within the desired frequency band of this study. The unshielded short cable insertion loss is $5.0 \mathrm{~dB}$ more than the shielded long cable. Further discussion on the effect of cable on IBC will be explored in chapter 5 . 

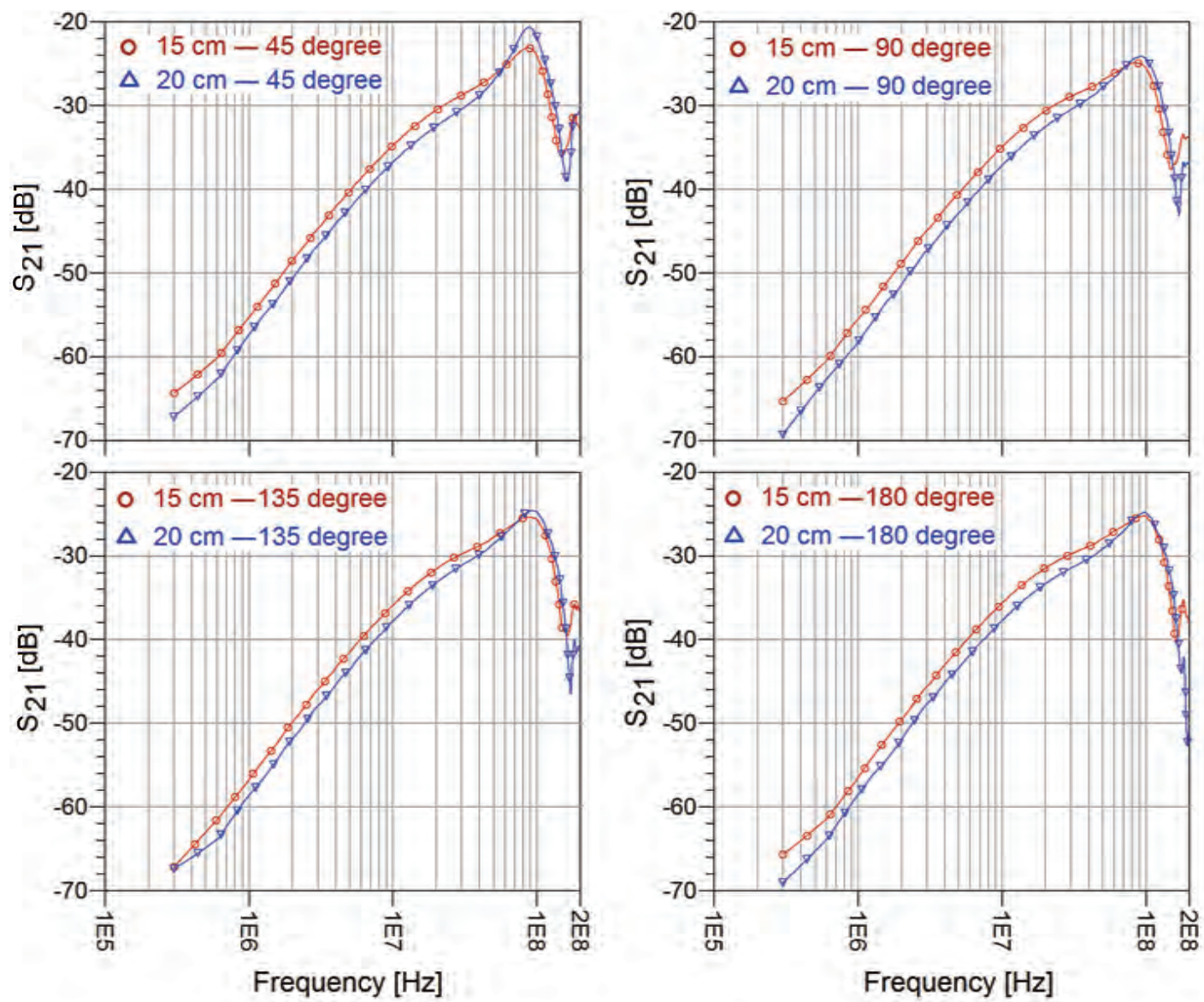

Figure 4.15: Variation of signal attenuation over input signal frequency. Each graph shows the results of 15 and $20 \mathrm{~cm}$ channel length for specified joint angles of 45, 90, 135 , and 180 degrees.

Our measurement results in section 4.2 showed that elbow joint flexion and extension lead to changes in signal attenuation in the two-electrode arrangement during constant on-body distance $(20 \mathrm{~cm})$ between transmitter and receiver electrodes. In this Section, the effects of both different distance and different joint angles, i.e., 45, 90, 135, and 180 degrees, were examined. Figure 4.15 depicts the influence of the 15 and $20 \mathrm{~cm}$ distances between transmitter and receiver electrodes during the joint angle of $45^{\circ}$ to $180^{\circ}$, respectively. 
The results indicate that the distance between transmitter and receiver also has significant effects on signal transmission when the arm is flexed or extended. For instance, at $2.0 \mathrm{MHz}$, the achieved signal attenuation was $48.5 \mathrm{~dB}$ in $15 \mathrm{~cm}$ in-body distance during 45 degree joint angle. However, for $20 \mathrm{~cm}$ distance between transmitter and receiver, this value was $50.6 \mathrm{~dB}$. That is longer communication channel leads to higher signal attenuation. Figure 4.16 shows the effects of distance on different joint angles in 2.0 and $60 \mathrm{MHz}$. According to results, at $2.0 \mathrm{MHz}$, due to the longer communication channel, the signal attenuation varies at least $2.0 \mathrm{~dB}$ in different joint angles. While at higher frequencies such as $60 \mathrm{MHz}$, the variations of attenuation is less than $2.0 \mathrm{~dB}$.

\subsubsection{Discussion}

Below $60 \mathrm{MHz}$ the results show less than $3.0 \mathrm{~dB}$ variation in attenuation between different limb positions. This is due to the fixed distances between transmitter and receiver electrodes on the human body. For frequencies above $60 \mathrm{MHz}$, the maximum difference of attenuation was found to be $2.0 \mathrm{~dB}$ between joint angles of 45 and 180 degrees. This could be due to the transmitter and receiver electrodes being physically closer together through the air (e.g. $5.8 \mathrm{~cm}$ for $45^{\circ}$ ) when the joint angle is smaller. Also, at higher frequency range the near field effect becomes a stronger return path which gives rise to lower attenuation.

The attenuation curves in figure 4.15 could be divided into two different regions according to frequency. The first area is from 0.3 to $60 \mathrm{MHz}$ where we observed that attenuation decreased at an approximately linear rate and was less affected by joint angles. This could be due to a majority of the signal power traversing the skin because of the decreasing penetration depth with frequency. The second area comprises the 


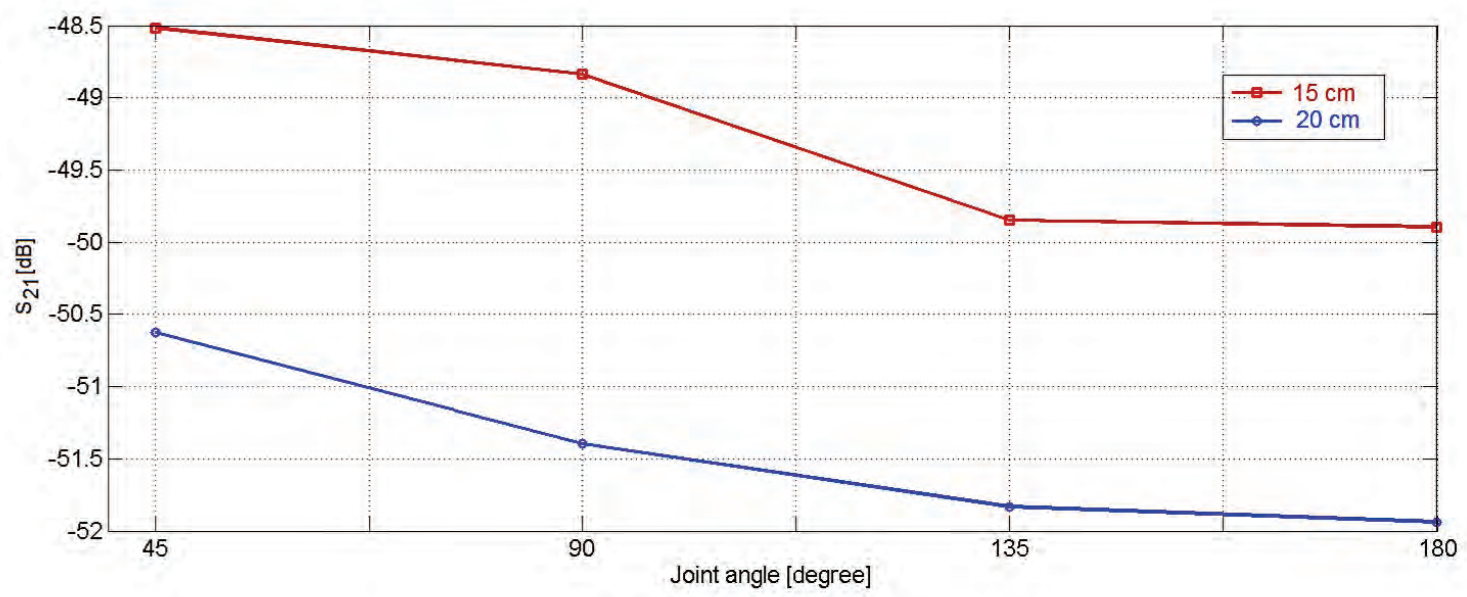

(a)

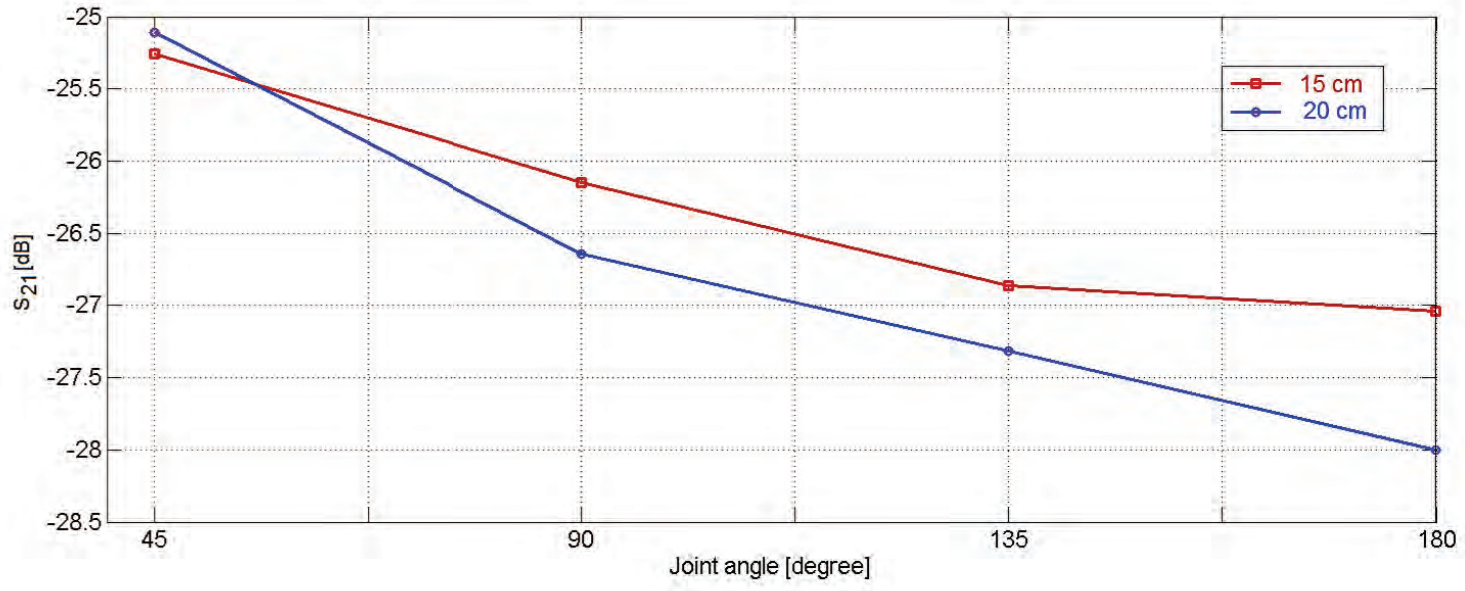

(b)

Figure 4.16: The value of average attenuations at different joint angles for two frequencies of (a) $2.0 \mathrm{MHz}$ and (b) $60 \mathrm{MHz}$ for channel lengths of $15 \mathrm{~cm}$ and $20 \mathrm{~cm}$.

minimum of attenuation which lies in the frequency range of 60 to $200 \mathrm{MHz}$ for different positions. This region could signify minimum penetration depth and hence joint effects (sudden changes in dielectric properties) are the most visible. In this area, the variations of signal attenuation do not follow a constant behavior potentially due 
to electromagnetic propagation. Different resonant frequencies (dependent on the limb characteristic) would explain the decrease in attenuation at interval frequency ranges. The results appear to generalize similarly across the three subjects tested.

The variation of attenuation is due to the flow of current through different tissue layers possessing differing dielectric properties. Since muscle has very high salinity and high water content, a good conductivity $(0.701 \mathrm{~S} / \mathrm{m}$ at $87 \mathrm{MHz}$ with minimum attenuation) is expected for muscle. Joint-segments do not contain a large mass of muscle and they are protected by cartilage which has even lower conductivity compared to muscle $(0.467 \mathrm{~S} / \mathrm{m}$ at $87 \mathrm{MHz})$ [94]. This could explain why the presence of a joint between transmitter and receiver increases the signal attenuation between two limbs by approximately $3.0 \mathrm{~dB}$ for transmission frequencies below $60 \mathrm{MHz}$. Based on figure 4.12, the sweet spot (minimum attenuation) in the presence of a joint would be below $60 \mathrm{MHz}$ region for IBC method.

\subsection{Environmental Effects on IBC}

Since human beings are in motion all the time, it is important to have a deep understanding of the possibility of the environment effects on IBC. In this section, we aimed to investigate possible effects of outdoor and indoor environment on the signal propagation through the body.

Measurements were conducted in three environments to investigate their effects on IBC. The same measurement setup and test subjects as section 4.1 were employed in this section. The transmitter and receiver electrodes were attached to subject's left leg (calf), $20 \mathrm{~cm}$ apart. Measurements were accomplished at the same time (11 am) on the three different days. 
Since the external ground has significant effect on IBC, particularly in two-electrode configuration on lower limb (see sections 4.1 and 4.2), the measurement has been carried out three times and in the multiple places. An electronic laboratory $\left(4 \times 6 \mathrm{~m}^{2}\right.$ in the ground floor) and a classroom on the $7^{\text {th }}$ storey $\left(3 \times 4 \mathrm{~m}^{2}\right)$ were selected as indoor areas. During the indoor measurements the temperature was in the range from $25^{\circ} \mathrm{C}$ to $28^{\circ} \mathrm{C}$. Several chairs and some electrical equipment were placed in the indoor areas. To evaluate an outdoor environment, experiments were carried out on a Rugby football field (on the grass) under ambient temperature of about $24^{\circ} \mathrm{C}$. The results in all three environments indicate similar signal attenuation behaviour for both electrode configurations.

\subsubsection{Results and Discussion}

Figure 4.17 and 4.18 shows the average and standard deviation of the channel loss calculated from all subjects during the indoor and outdoor experiments. The length of the body communication channel was fixed to $20 \mathrm{~cm}$ while the electrodes attached to the subject's calf. The evaluation of physical ground effects confirms that the frequency range of 40-60 MHz gives the minimum path loss in IBC for all experiment environments. Results show that the variation of the measurement environment (i.e., various external grounds) causes less than $2.0 \mathrm{~dB}$ path loss for both electrode configurations for frequencies up to $50 \mathrm{MHz}$. This could be explained by the propagation of the signal occurring mainly through the tissue layers, below $50 \mathrm{MHz}$. Further, the on-body electrodes have the same distance from the physical ground. Based on the achieved results, the highest standard deviation is obtained during the indoor measurement at the ground floor (electronic laboratory) within the frequency range of 35-40 MHz. That is, $5.0 \mathrm{~dB}$ and $4.2 \mathrm{~dB}$ are achieved for two- and four-electrode 

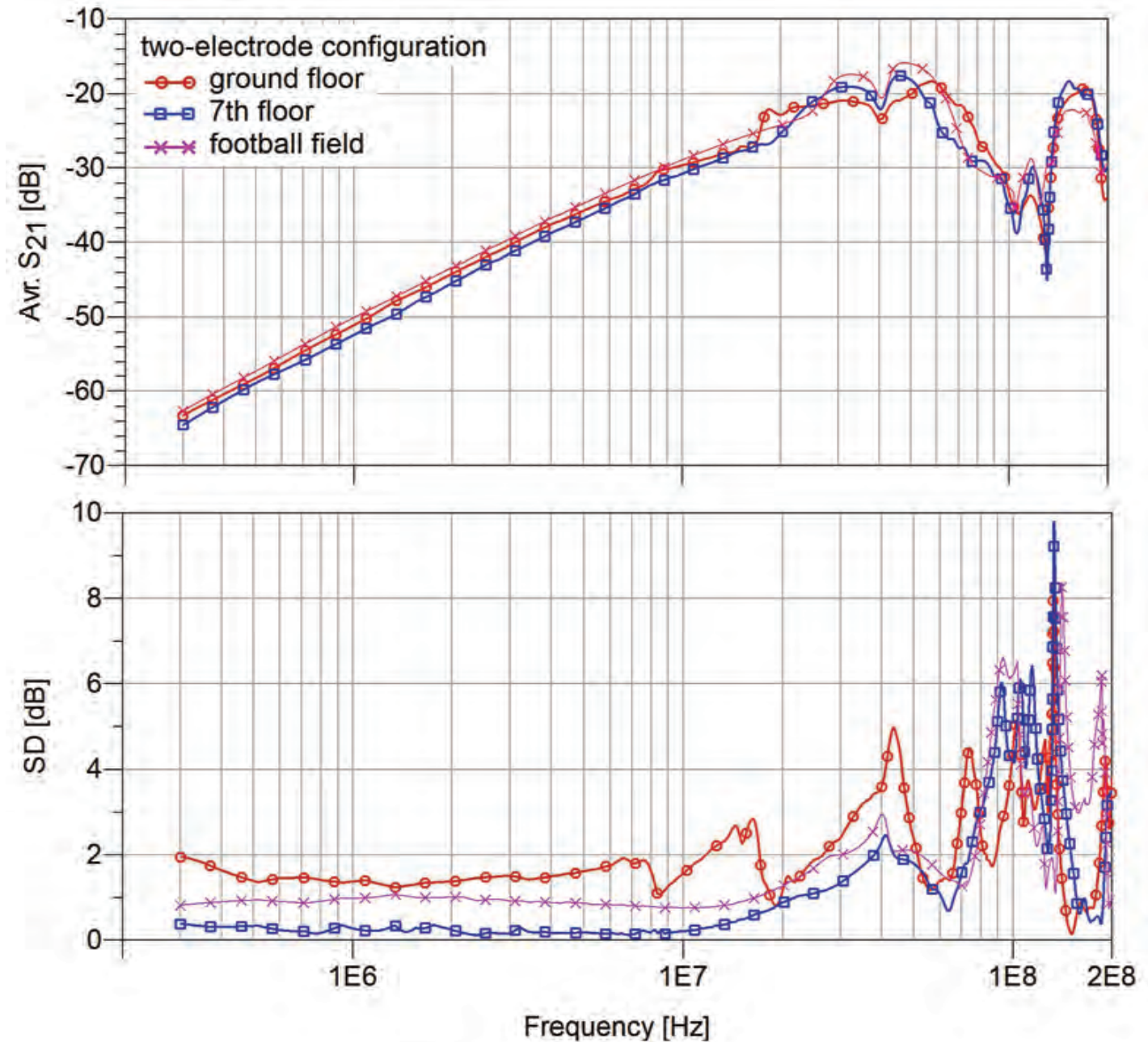

Figure 4.17: The average (Avr.) and standard deviation (SD) of the IBC channel loss in three different environments for two-electrode configurations.

configuration, respectively. This suggests that the influence of indoor electronics interference on the IBC signal transmission is not negligible [22].

The observed resonance frequency at approximately $100 \mathrm{MHz}$ could be due to the effects of our measurement equipment such as cables. According to Xu et al. [22], the electromagnetic radiation from the connecting cables become significant as frequency increase. In conclusion, the minimal influence of different surrounding environments 

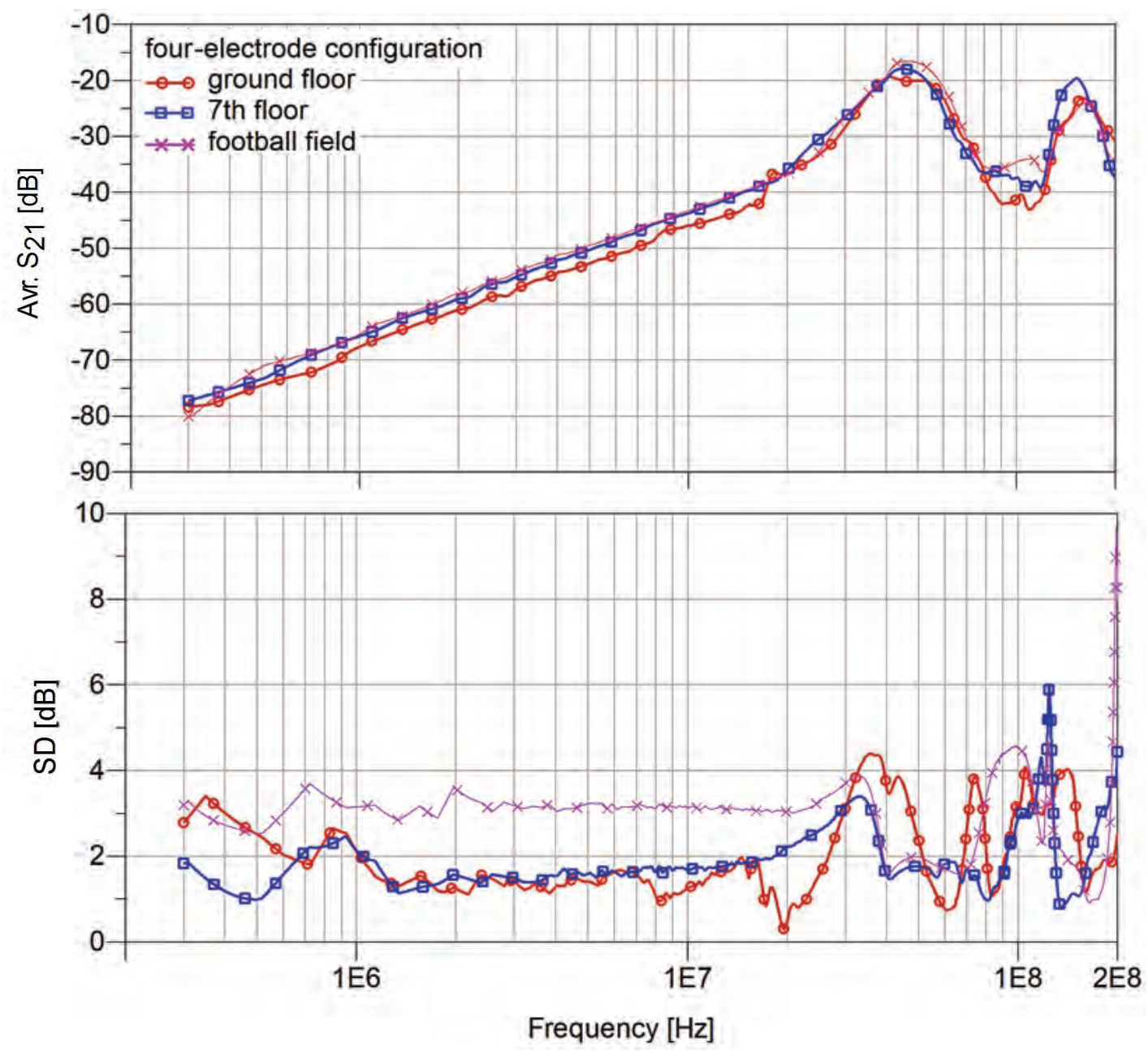

Figure 4.18: The average (Avr.) and standard deviation (SD) of the IBC channel loss in three different environments for four-electrode configurations.

at the frequency band $0.3-50 \mathrm{MHz}$ demonstrates the promising potential of applying IBC for both indoor and outdoor medical applications.

\subsection{Summary}

In this chapter, comprehensive experiments have been carried out to understand the effects of joint-segments on signal propagation through the human body. The 
measurements investigated the presence of two influential factors, namely the presence of joints in a signal path and the joint angles. Results show that the presence of joints along transmission path causes high signal attenuation (up to $6.0 \mathrm{~dB}$ in the fourelectrode configuration). The knee joint shows higher attenuation (more than $2.0 \mathrm{~dB}$ ) compared to the elbow joint, below $60 \mathrm{MHz}$. Second, different joint angle effects have been illustrated so as to have deeper understanding of the body limb movements on IBC signal transmission. The flexed joint exhibits $4.0 \mathrm{~dB}$ less attenuation compared to the extended one. Results also indicate that the influence of different surrounding environments is negligible at the frequency band of 0.3-50 MHz. The investigations on the two- and four-electrode configurations indicate that two-electrode configuration is more susceptible to body movement (particularly in the upper limbs) at frequencies less than $60 \mathrm{MHz}$ but less susceptible when deployed on the lower limbs.

The results also suggest that the IBC systems that implement IEEE 802.15.6 need to take the potential variation of signal attenuation during complex body motion such as walking into account. The next chapter will focus on the development of more accurate posture-related circuit models to explain the observed empirical results. 


\section{Chapter 5}

\section{Body Channel Modeling}

The communication channel modeling provides researchers with a profound understanding of data transmission mechanism over a body channel [62]. Due to the complicated and complex anatomy of the human body, modeling the human body as a communication medium is a challenging but demanding task for future IEEE 802.15.6 standard design and performance optimization [87]. One of the significant challenges with respect to the RF WBANs (NB and UWB) when employed in portable devices is the variations in channel characteristics such as multipath propagation, body shadowing effects, and time-varying channel as a result of user movement. WBAN communications should be robust and flexible during body motion especially if they are to be successfully deployed in everyday life. Although some studies have examined the body motion effects on RF WBAN systems [88] by means of channel modeling techniques such as numerical and theoretical simulation, the model of body motion in IBC considering limb structure have not been proposed, particularly for wider frequency bands (0.3-200 MHz).

Since human body joints have a significant role in movement, new IBC circuit 
models explaining joint effects with a focus on the elbow joint are proposed in this chapter for the first time. In fact, the model of body arm involving elbow joint as the heterogeneous material is necessary; because it is the only way to independently analyze the effect of joint-segment on IBC method. Moreover, the limb joint consideration in the modeling approach may reproduce different aspects that are caused by the moving body. Our proposed models also take into account the influence of measurement equipment on signal attenuation within a higher frequency range i.e., 0.3-200 $\mathrm{MHz}$ compared to the models of previous studies (less than $100 \mathrm{MHz}$ [59]). The IBC empirical measurements on real human body in chapter 4 provided empirical evidence that limb joints affect the signal behavior while using IBC. This chapter is organized as follows: section 5.1 briefly reviews background of the current IBC models. Section 5.2 investigates the anatomy of human arm and effects of tissue dielectric properties on the signal transmission. A proposed model for human arm communication channel is presented in the last part of section 5.2. Section 5.3 describes the measurement setup and the achieved results from both empirical and simulation of the communication channel. This is followed by the comparison between the measurements and model predictions in section 5.4. Finally, section 5.5 and 5.6 contain the discussions of the obtained results, conclusion and summary of the chapter, respectively.

\subsection{Background on IBC Channel Models}

The first model of body communication channel using electrical lumped elements was proposed by Zimmerman [39]. This equivalent circuit model consists of four impedances in between IBC transmitter and receiver electrodes which represented lumped electrical model representations of human tissue. However, more detailed 
components such as inter-electrode impedances were ignored in this preliminary model. In 2011, more than a decade after Zimmerman's model following several attempts to propose an accurate IBC model $[20,46,54]$, a new simplified circuit model was introduced by Song et al. [57]. The authors added the output resistance of transmitter $(50 \Omega)$ as well as the input impedance of receiver $(1 \mathrm{M} \Omega$ and $13 \mathrm{pF})$ to improve the proposed model by Wegmueller et al. in 2007 [20]. The comparison between the measurement and simulation results indicated that the absolute error in the operation frequency range $0.1-5 \mathrm{MHz}$ was limited to $2.0 \mathrm{~dB}$. Their model did not consider the effects of body joints as well as the measurement equipment used for removing the influence of common ground e.g., balun. Hence, a high correction factor $(=20.2 \mathrm{~dB})$ was required to fit the model simulations results to empirical values. Recently, a theoretical model of IBC was proposed based on the equation of electric field (near-field and far-field) [62]. The comparison of the empirical and modeling results for body channel loss verified the validity of the IBC model for a frequency range of $0.1-100$ $\mathrm{MHz}$ and the channel length from 10 to $130 \mathrm{~cm}$. However, this model covered only the wave propagation IBC method, i.e. transmission through human skin surface. Therefore, only the characteristics of skin layer were involved in the model and inner tissue layer effects were ignored. Moreover, the effects of measurement components such as baluns were not considered in the model. In the mentioned models, the human body was approximated as a homogeneous solid volume and more detailed segments such as body joints were rarely taken into account.

According to the achieved results in chapter 4, the presence of joint along the transmission path caused up to $6.0 \mathrm{~dB}$ higher signal attenuation. Hence, the joint influences on signal transmission need to be considered in the human body channel modeling based on dielectric properties of biological tissues. In this chapter, we aim 
to develop a new equivalent circuit model of a human arm including elbow joint impedance elements. The new model predicts intrabody signal propagation behavior in higher frequency range of 0.3-200 MHz. A root mean square error (RMSE) of only $0.25 \%$ implies the high accuracy of the models. Our proposed models explain the non-negligible effects of IBC measurement equipment at higher frequencies.

\subsection{Methods}

In this Chapter, four on-body electrodes are employed to differentially transmit current into the human tissues. Since the signal frequency range in our study $(0.3-200$ $\mathrm{MHz})$ is much higher compared to the common galvanic coupling IBC ( $<2 \mathrm{MHz}$ ) in past studies [27], we refer to the setup as a four-electrode configuration rather than the galvanic coupling [20]. Figure A.1 shows pathways for a current flow between the transmitter and receiver electrodes in the four-electrode configuration. A major portion of current propagates between the transmitter electrodes and a smaller current is received by the two receiver electrodes. Based on the operation frequency, the smaller current propagation follows two paths as seen in figure A.1. Path 1 is through human body where the amount of current arriving at receiver depends on the human tissue dielectric properties. At frequency of higher than tens $\mathrm{MHz}$, we propose that the signal is coupled more through the air capacitance (path 2) between on-body electrodes or connecting cables. At even higher frequencies ( $>60 \mathrm{MHz}[62])$, the signal may start to radiate from the measurement equipment and the body skin (the body antenna effects). Thus, in this chapter, an equivalent circuit model of the human body (the arm) is developed for the first time using four-electrode configuration which examines the body channel characteristics in the presence of joint-segment along the 

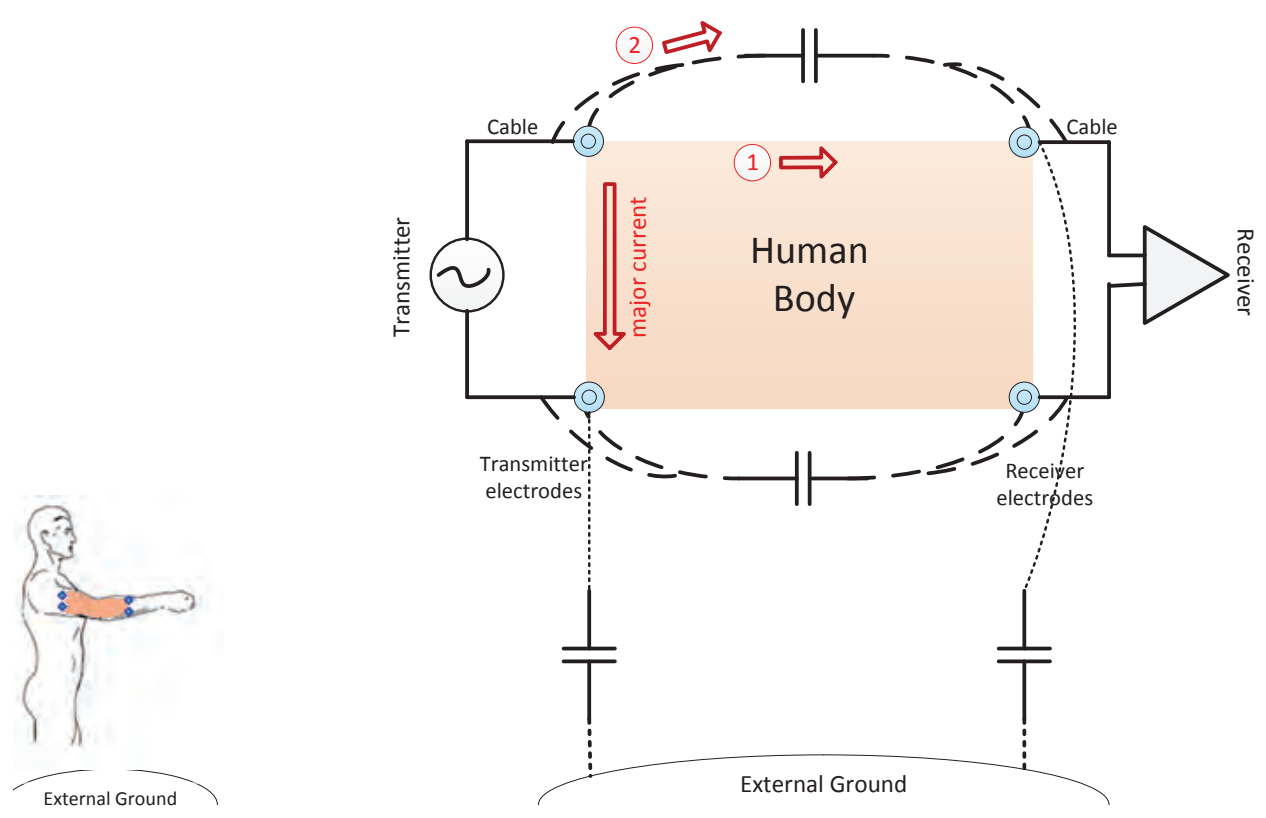

Figure 5.1: Current propagation through body and air, current path 1 and 2 respectively, during four-electrode IBC configuration

signal transmission path. Unlike previous studies which simulated the IBC below $100 \mathrm{MHz}$ only [62]- [59], this work developed the model within the higher operating frequency range of 0.3-200 MHz.

\subsubsection{Anatomy of the Human Arm}

The human arm consists of three main segments, the upper arm between shoulder and elbow, the forearm between elbow and wrist, and the hand [30]. In addition, there are four different joint-segments: the shoulder joint, the elbow joint, the wrist joint, and the radioulnar joint. Body movements are mainly permitted by joints where two bones make contact. For instance, elbow joint is the linkage of the upper arm bone (humerus) with forearm bones i.e., ulna and radius. Since anatomy of joint is slightly different from other limb segments, this has to be considered in human 


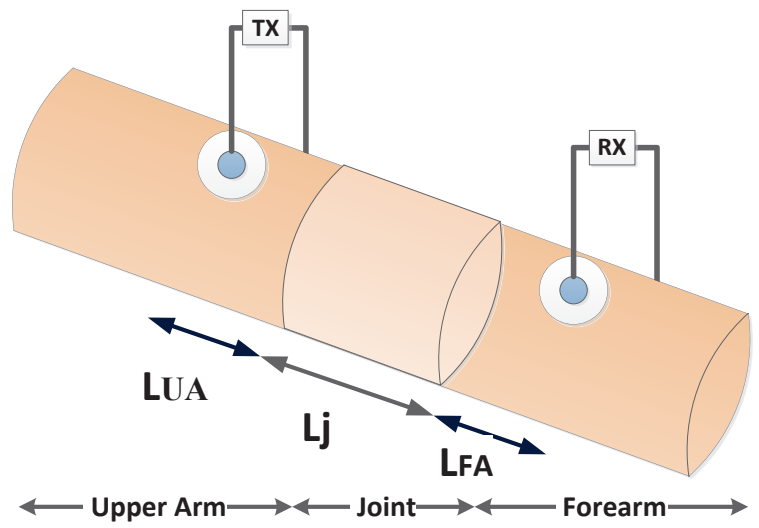

(a)

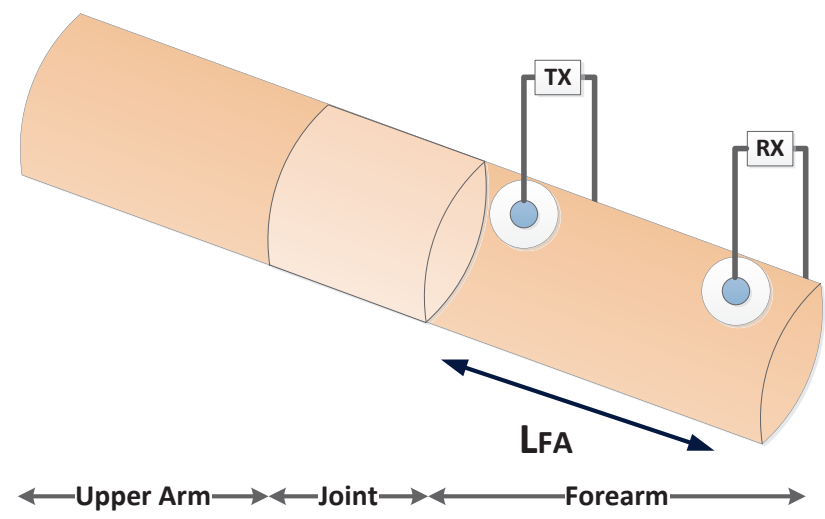

(b)

Figure 5.2: Geometrical human arm model including four on-body electrodes and presence of the elbow joint in the middle of signal transmission path between transmitter (TX) and receiver (RX) electrodes. (a) Human body communication channel is involved the limb joint (b) No joint is across the data transmission path

channel modeling to analyze the IBC method.

Control of the signal propagation, reflection, and attenuation through the human body like any other conductor is mainly dependent on the dielectric properties (relative permittivity and conductivity) of body tissue layers. According to Kanai 
et al. [52], the characteristics of human tissues can be modeled as a time constant equivalent circuit composed of resistance and capacitance (more details in chapter 2). In IBC, if the longitudinal distance between electrodes is fixed to $d$ and $A$ represents the cross section area of each tissue layers, then the complex impedance of the $R C$-parallel network of the tissue layers among the electrodes can be calculated as follows:

$$
\begin{gathered}
C(W)=\varepsilon(\omega) \frac{A}{d} \quad, \quad G(W)=\sigma(\omega) \frac{A}{d} \\
Y^{*}=Y_{C}+Y_{G}=\sigma^{*} \frac{A}{d} \quad, \quad Z^{*}=\frac{1}{Y^{*}}
\end{gathered}
$$

where $Y^{*}$ and $\sigma^{*}=\sigma+\mathrm{j} \omega \varepsilon_{0} \varepsilon_{r}$ are the complex admittance and the complex conductivity, respectively. The relative permittivity $\left(\varepsilon_{r}\right)$ and electrical conductivity $(\sigma)$ of human tissues are reported online based on Gabriel's research findings [1].

In our proposed circuit model for human arm, the geometry of the human arm is approximated as a non-homogeneous cylinder with length $L_{A}$ and the diameter $D_{A}$. It is segmented into three blocks which are the upper arm, forearm, and elbow joint in the middle of cylinder $\left(L_{A}=L_{U A}+L_{j}+L_{F A}\right)$. While upper arm and forearm possess equal length $\left(L_{U A}=L_{F A}\right)$, they have different length from elbow joint $\left(L_{j}\right)$. It is assumed that the upper arm and forearm consist of various tissue layers such as skin, fat, muscle, and bone with different electrical properties and thickness. The elbow joint is a complex structure comprised of multiple layers such as skin, fat, ligament, tendon, cartilage, and bone. Joints are protected by cartilage and they have smaller amount of muscle compared to other parts of human body. Tendons of several muscles cross the joint and provide limb movements. Meanwhile, joints are reinforced with ligaments which connect one bone to another [30]. Figure 5.2 shows the proposed 


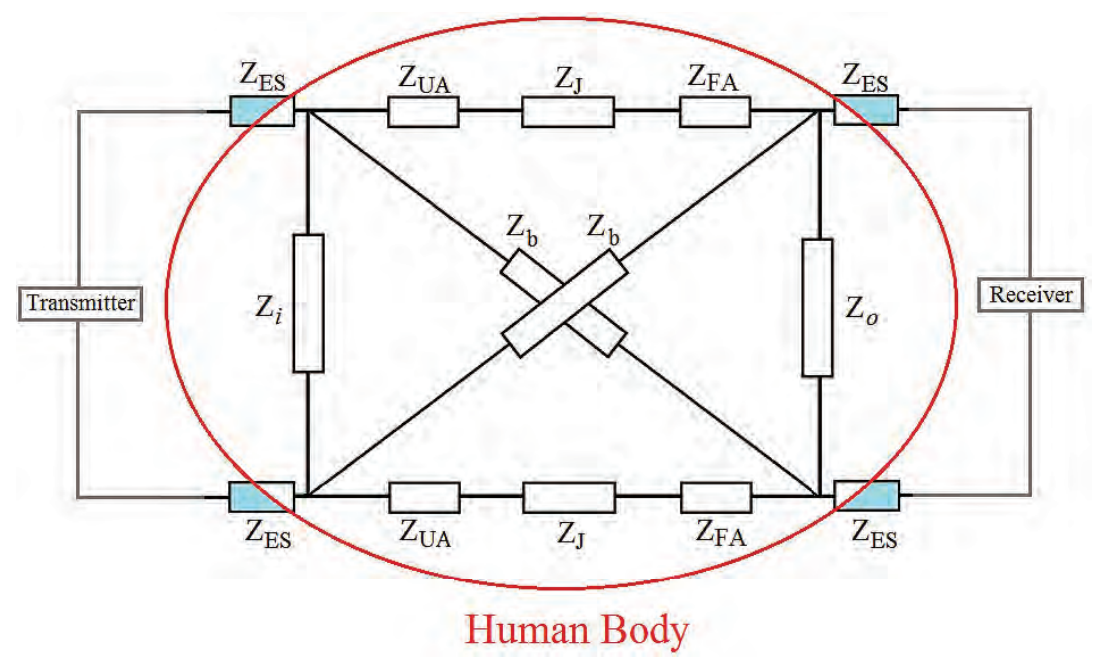

Figure 5.3: Proposed circuit model of human arm considering elbow joint during four-electrode IBC configuration: Circuit model A.

geometrical model of human arm when the four-electrode configuration is used.

\subsubsection{Limb-Joint Circuit Model}

Our proposed model (figure 5.3) takes into account the effect of human elbow joint, which was not done in previous studies on body circuit models $[20,54,60]$. Three series impedances $Z_{U A}, Z_{J}$, and $Z_{F A}$, are used to represent the human upper arm, elbow joint, and forearm impedances, respectively. Transversal current flows between transmitter electrodes and receiver electrodes. The transversal impedances are modeled by $Z_{i}$ and $Z_{o}$. Cross impedances between transmitter and receiver electrodes are modeled by $Z_{b}$. Since electrodes act as interface between transmitter or receiver and human body, the impedance of skin-electrode $\left(Z_{E S}\right)$ is also considered in the proposed model.

To calculate the value of the impedances based on (5.1) and (5.2), the electrical properties as well as the different tissue layer sizes have to be considered. For instance, 


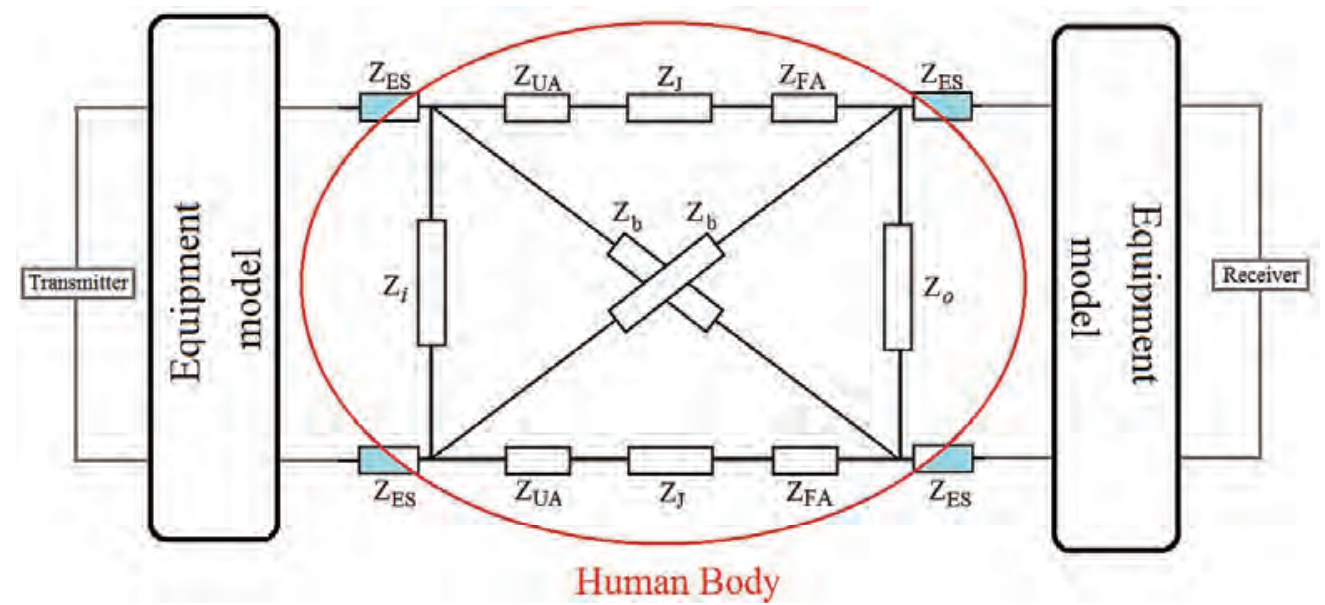

Figure 5.4: The body channel circuit model including measurement equipment model: Circuit model B.

Table 5.1 expresses the thickness of tissue layers for both no joint and joint-segments such as skin related to one of the participants in this study. The values are calculated in proportion to the tissue layer thicknesses mentioned in the Wegmueller's model [9]. The elbow joint area is divided into three main tissue layers including bone, skin, and fat. The bone layer, including cartilage and bone marrow, comprise half of the elbow joint [89]. Similar to the forearm and upper arm, we assume the same thickness for skin and fat tissue in the joint-segment [72]. It should be noted that the thin layer including the muscle, tendon, and ligament is also considered in the joint-segment. $Z_{U A}, Z_{J}$, and $Z_{F A}$ are the equivalent of parallel impedances of different tissues while the series impedances of tissues consist of $Z_{i}$ and $Z_{o}$. It is worth noting that, because of the high operation frequency range, the effects of measurement equipment should be considered in the proposed model (see figure 5.4). 
Table 5.1: Thickness of the Tissue Layers Based on Human Arm Radius

\begin{tabular}{|l|c|c|c|c|}
\hline \multicolumn{2}{|c|}{ no joint-segment (Forearm and Upper arm) } & \multicolumn{2}{c|}{ joint-segment } \\
\hline \hline $\begin{array}{c}\text { Tissue } \\
\text { layer }\end{array}$ & $\begin{array}{c}{[9]} \\
\text { Arm radius } \\
=50 \mathrm{~mm}\end{array}$ & $\begin{array}{c}\text { test subject } \\
\text { Male }(\# 1) \\
\text { Arm radius } \\
=36 \mathrm{~mm}\end{array}$ & $\begin{array}{c}\text { Tissue } \\
\text { layer }\end{array}$ & $\begin{array}{c}\text { test subject } \\
\text { Arm }(\# 1) \\
=36 \mathrm{~mm}\end{array}$ \\
\hline Skin & 1.5 & 1.08 & Skin & 1.08 \\
\hline Fat & 8.5 & 6.12 & Fat & 6.12 \\
\hline Muscle & 27.5 & 19.8 & $\begin{array}{c}\text { Muscle, } \\
\text { tendon, and } \\
\text { ligament }\end{array}$ & 5.76 \\
\hline $\begin{array}{c}\text { Cortical } \\
\text { bone }\end{array}$ & 6.0 & 4.32 & $\begin{array}{c}\text { Cortical } \\
\text { bone }\end{array}$ & 5.76 \\
\hline $\begin{array}{c}\text { bone } \\
\text { marrow }\end{array}$ & 6.5 & $\begin{array}{c}\text { Cartilage } \\
\text { and Bone } \\
\text { marrow }\end{array}$ & $\begin{array}{c}17.28 \\
\text { (radius) }\end{array}$ \\
\hline
\end{tabular}

\subsection{Measurement and Simulation}

As shown in figure 5.3, the human arm is modeled using equivalent circuit model to simulate the changes in the communications channel while the elbow joint is located along the current pathway through the body. Comparisons between empirical measurements and simulation results will validate the accuracy of the proposed model.

Attenuation has a crucial role in an efficient IBC transceiver design. In this study, we investigated the joint presence effect as a parameter in relation to channel attenuation. The channel attenuation generally varies with frequency and can be measured using S-parameters. The $S_{21}$ transmission coefficient represents the attenuation between the transmitter and the receiver electrodes. $S_{21}$ of human body channel is expressed by:

$$
S 21_{\text {body channel }}=20 \log _{10}\left(\frac{V_{\text {receiver }}}{V_{\text {transmitter }}}\right)
$$




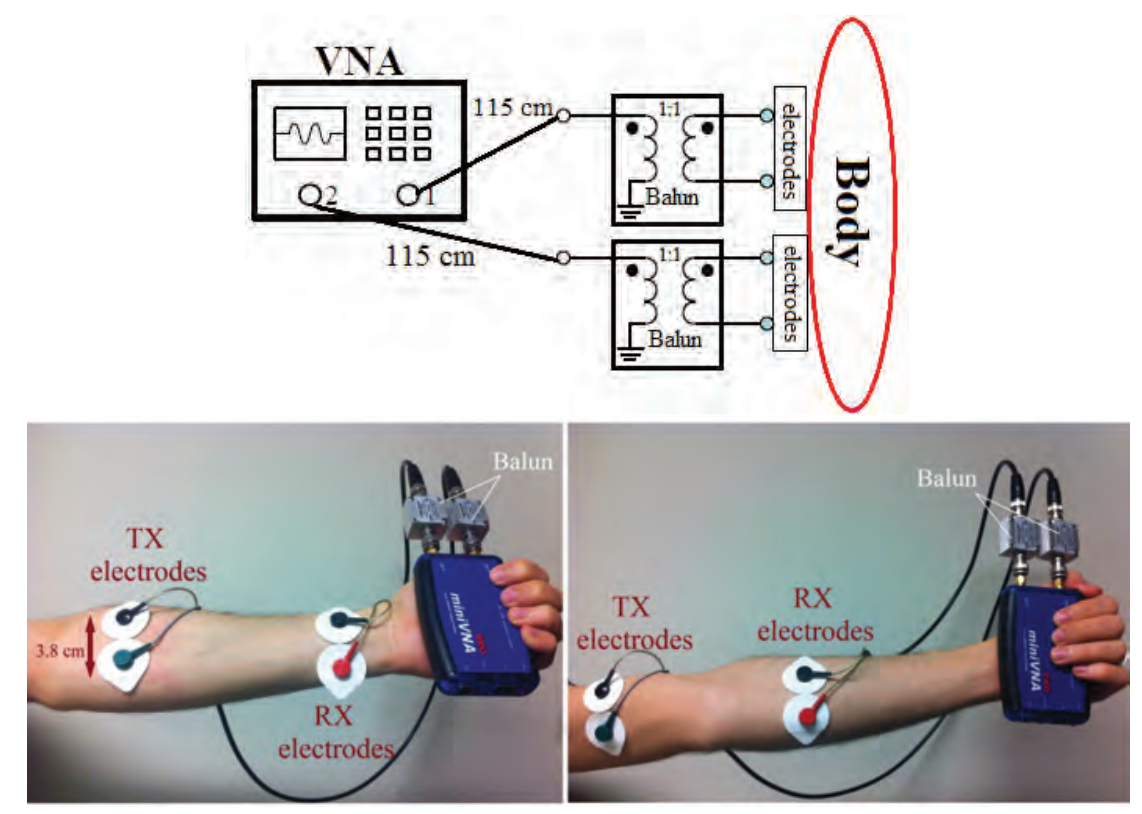

Figure 5.5: The measurement setup of IBC using four on-body electrodes during no joint (left) and with joint (right) positions.

where $V_{\text {receiver }}$ is the received voltage amplitude and $V_{\text {transmitter }}$ the transmitted voltage amplitude.

\subsubsection{Empirical Measurement}

The experimental architecture of the four-electrode IBC configuration is shown in figure 5.5. Four commercial $\mathrm{Ag} / \mathrm{AgCl}$ single electrodes (Noraxon Inc., Scottsdale, AZ, USA), EMG shielded snap leads as connecting cables, MiniVNA Pro, and a pair of balun transformers placed between the on-body electrodes and MiniVNA Pro ports were employed for our empirical measurement. The measurement equipment details have been explained in chapter 3 .

To measure the signal attenuation, port 1 of miniVNA was considered as the transmitter and port 2 was used as the receiver. The miniVNA was calibrated (using 
standard calibration kits) to transmit a low power test signal to the human body below $0 \mathrm{dBm}$. The measured attenuation by mini VNA is the sum of the human body channel attenuation (equation 5.3) and measurement equipment attenuation such as connecting cables and baluns [90].

Four healthy subjects, one female and three males, are volunteered to participate in this study. The physical parameters of the subjects are: 26 to 33 years old, 172 to $183 \mathrm{~cm}$ height, and 60 to $85 \mathrm{~kg}$ weight. Considering an average weight of 65 $\mathrm{kg}$, the maximum safe transmission power would be $37 \mathrm{dBm}(=5 \mathrm{~W})$ [84]. The miniVNA output power (source power) was fixed to a low power of $0 \mathrm{dBm}(=1.0$ $\mathrm{mW}$ ) for further safety. The sweep signal frequency range was set from 0.3 to $200 \mathrm{MHz}$ with a $50 \Omega$ impedance MiniVNA Pro. In all experiments, we set up four-electrode IBC configuration, where the signal was differentially applied at the transmitter and receiver sides. All experiments were repeated three times over three sequential days and the average data were used in the analysis.

In the first measurement, the receiver electrodes were attached on the subject's left forearm and the transmitter electrodes were positioned on the upper left arm to measure the influence of the joint-segment. As in figure 5.2, the distance between transmitter and receiver electrodes is divided to three segments with lengths denoted by $L_{U A}, L_{j}$, and $L_{F A}$. The length of elbow joint $\left(L_{j}\right)$ was assumed $8.0 \mathrm{~cm}$ in both empirical and simulation measurements. In addition, the distance of transmitter electrodes from elbow joint was equal to the distance of elbow joint to receiver electrodes $\left(L_{U A}=L_{F A}\right)$.

In the next measurement, the electrodes of both transmitter and receiver were attached to the subject's left forearm. The receiver electrodes were close to the wrist, therefore, no joint was in the transmission path (figure 5.5). During both experimental 

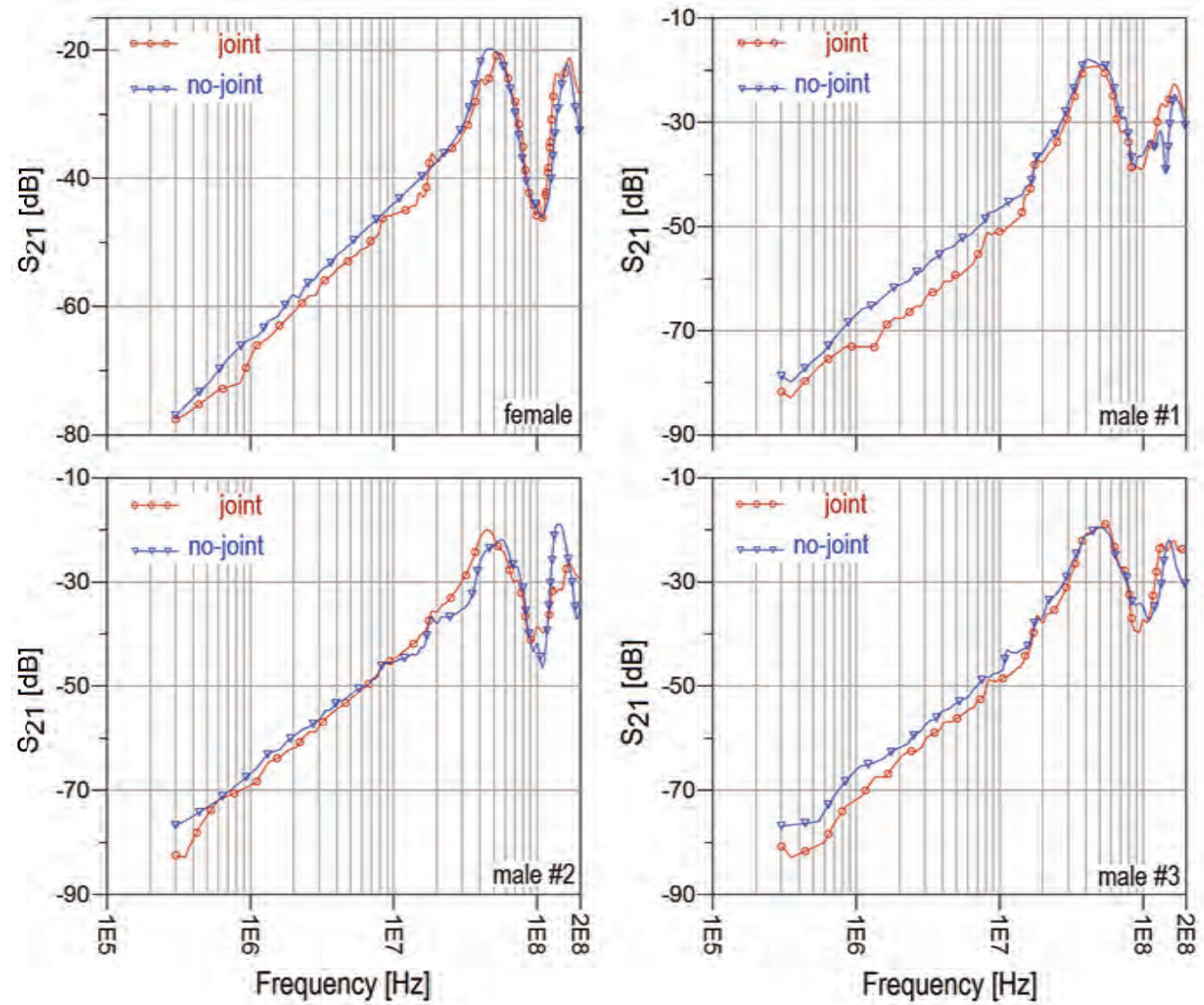

Figure 5.6: The results of empirical measurements on a body arm using IBC.

conditions the distance between the centers of the electrodes was set to $20 \mathrm{~cm}$. The subject was asked to stand with left arm straight out in front of the chest and right arm at the side in a relaxed position. At each measurement position, the attenuation was measured for the frequency range of 0.3 to $200 \mathrm{MHz}$.

Figure 5.6 shows the measured signal attenuation for a $20 \mathrm{~cm}$ communication channel distance for four different subjects. Results indicate that the presence of a joint between transmitter and receiver electrodes causes 1.0 to $5.2 \mathrm{~dB}$ higher signal attenuation compared to the no joint situation. These variations of attenuation 
are observed in the frequency range of 0.3 to $20 \mathrm{MHz}$. Increasing the frequency of operation leads to decreasing differences between attenuations observed in joint and no-joint scenarios. Overall, it was found attenuation decrease gradually with increasing frequency for all subjects up to approximately $54 \mathrm{MHz}$.

\subsubsection{Circuit Model Simulation}

"Advanced design system (ADS)" software (Agilent EEsof EDA Inc., Santa Rosa, CA, USA) was utilized to simulate the proposed human arm circuit models (figure $5.3,5.4)$. In this simulation method, two circuit models were examined. In model A, the body circuit model was directly connected to a signal source and VNA, while the effect of baluns and cables was not taken into account. Moreover, the S-parameters of differential circuit model were measured without using baluns due to the use of VNA with ungrounded ports. The first schematic is illustrated in figure 5.7(a). In the second model, the possible influence of cables as well as baluns was modeled by $R L C$ components and importing the S-parameter data file of balun (s2p file [90]), respectively (see figure 5.7(b)). It is worth noting that, S-parameter simulation is used to calculate the channel attenuation rather than the network analyzer with ungrounded ports in the circuit model B. In both models simulation, the impedance of VNA ports was considered as $50 \Omega$.

According to (5.1), to calculate the component values of $Z_{U A}, Z_{J}$, and $Z_{F A}$, in addition to the geometries of the subjects, the length of upper arm, joint, and forearm are necessary (see Table 5.2).

The value of $Z_{i}$ and $Z_{o}$ are highly affected by inter-electrode distance (distance between two electrodes on one side $=3.8 \mathrm{~cm}$, see figure 5.5 ) and diameter of the employed electrodes $(1 \mathrm{~cm})$. As seen in figure 5.8, the larger intra-electrode distance 


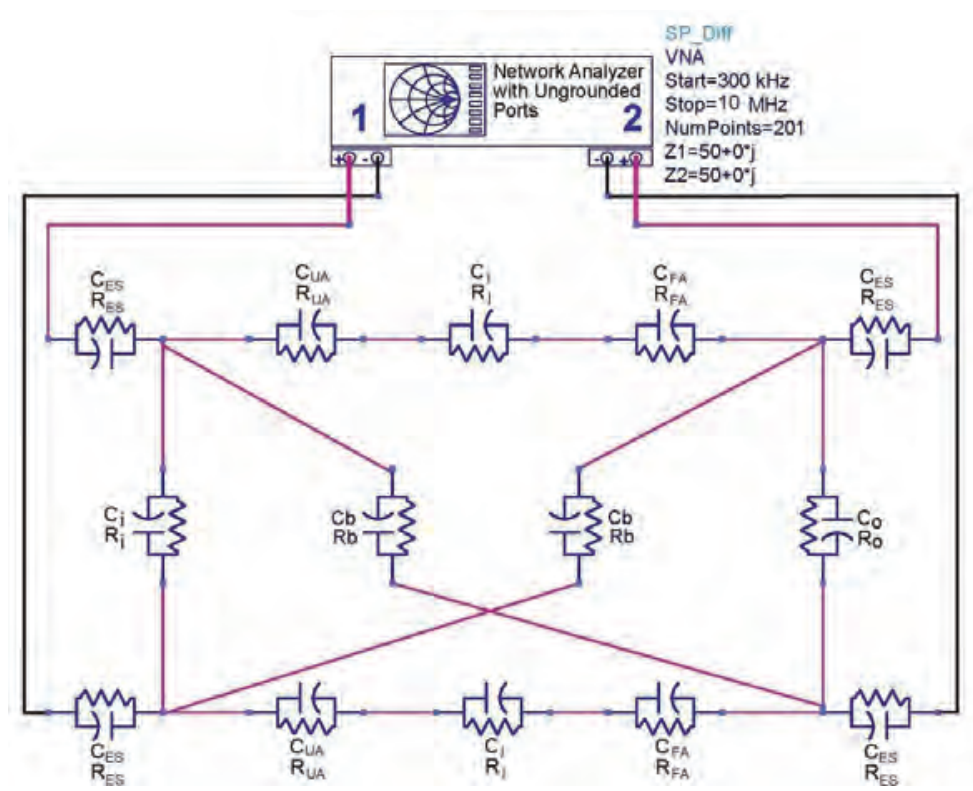

(a)

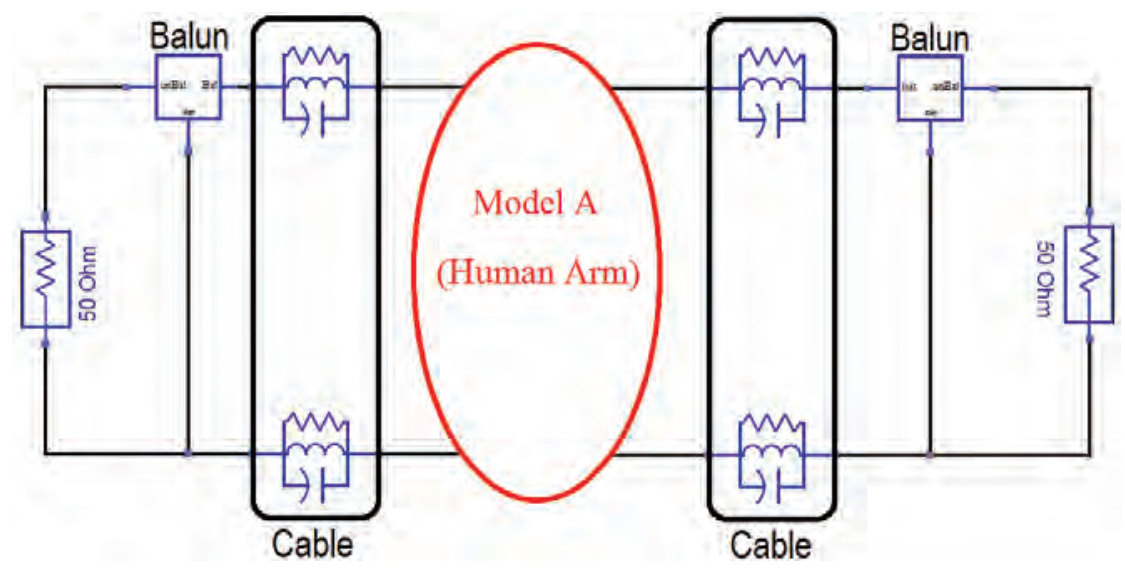

(b)

Figure 5.7: (a) Circuit model A: simulation of the human arm in presence of the elbow joint. (b) Circuit model B: simulation by adding the cables and Baluns model.

(between Tx and Rx) results in deeper penetration of current into the inner tissue layers. Owing to the short distance between two circular conductive area of electrodes $(=1.0 \mathrm{~cm})$ and also higher conductivity of wet skin compared to subcutaneous fat and bone, the transversal current approximately distributes between electrodes through 
Table 5.2: The Geometries of the Subjects and the Length of the Communication Channel

\begin{tabular}{|c|c|c|c|c|c|}
\hline Subject & $\begin{array}{c}\text { Arm Diameter } \\
\left(D_{A}\right)\end{array}$ & $\begin{array}{c}\text { Distance between } \\
\text { arm and } \\
\text { external ground }\end{array}$ & $L_{U A}$ & $L_{J}$ & $L_{F A}$ \\
\hline \hline Female & $6.4 \mathrm{~cm}$ & $138 \mathrm{~cm}$ & $6 \mathrm{~cm}$ & $8 \mathrm{~cm}$ & $6 \mathrm{~cm}$ \\
\hline Male$(\# 1)$ & $7.2 \mathrm{~cm}$ & $149 \mathrm{~cm}$ & $6 \mathrm{~cm}$ & $8 \mathrm{~cm}$ & $6 \mathrm{~cm}$ \\
\hline Male(\#2) & $7.8 \mathrm{~cm}$ & $152 \mathrm{~cm}$ & $6 \mathrm{~cm}$ & $8 \mathrm{~cm}$ & $6 \mathrm{~cm}$ \\
\hline Male(\#3) & $8.9 \mathrm{~cm}$ & $153 \mathrm{~cm}$ & $6 \mathrm{~cm}$ & $8 \mathrm{~cm}$ & $6 \mathrm{~cm}$ \\
\hline
\end{tabular}

the wet skin layer only. Therefore, the following equation was used to calculate $Z_{i}$ and $Z_{o}$ values:

$$
Z_{i}=Z_{o}=\frac{2 l_{\text {skin }}}{A_{\text {electrodes }}\left(\sigma_{\text {skin }}+j \omega \varepsilon_{0} \varepsilon_{r \text { skin }}\right)}
$$

where $l_{\text {skin }}$ is the thickness of skin layer. Additionally, the value of $Z_{b}$ is determined by the sum of the transversal and longitudinal impedances [57]:

$$
Z_{b}=Z_{i}+Z_{U A}+Z_{J}+Z_{L A}
$$

Therefore, the most effective impedance to determine body channel attenuation is the contact impedance or $Z_{E S}$ [55]. The value of $Z_{E S}$ is represented by a series of three impedances consisting of electrode metal impedance, gel impedance, and skin layer impedances which include epidermis and subdermal impedances. According to Besio et al. [56], in the suitable contact between electrode and skin surface, the amount of $Z_{E S}$ is less than $10 \mathrm{k} \Omega$. To achieve the value of $Z_{E S}$ in this study, port 1 of Mini VNA Pro is directly connected to the on-body transmitter electrodes via the connecting cable. It should be noted that the impedance of both transmitter and receiver loads were considered in our model simulation. Figure 5.9 plots the real and imaginary of 


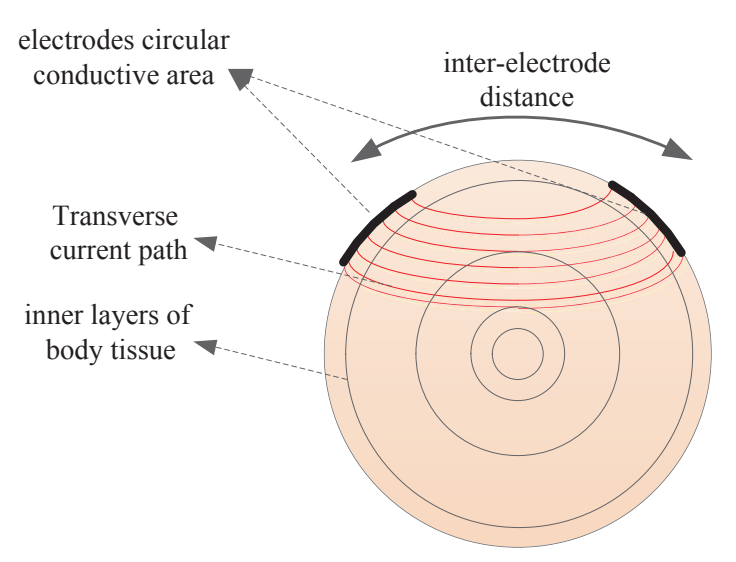

Figure 5.8: The transverse current flow between two electrodes of transmitter or receiver through the inner layers of human tissue.

the measured $\mathrm{Z}$ which is a combination of three series impedances including cable, as follows:

$$
Z=Z_{E S}+Z_{i}+Z_{E S}
$$

where $Z_{i}$ is the input impedance. Further, the influence of the connecting cable needs to be considered in order to develop a more precise model. Based on figure 5.9, two resonance points are observed around 90 and $180 \mathrm{MHz}$. This could be explained by the resonance of the employed shielded cable at higher frequencies. While a shielded cable has both resistance and inductance, a capacitance could be created between the signal conductors of cable [92]. Therefore, the parallel $R L C$ lumped circuits are employed to simulate the behaviors of the connecting cables in our simulation. The reflection coefficient, $S_{11}$, is used to determine the $R L C$ circuits. To this end, the cable is connected to the port 1 of the MiniVNA Pro while the far end of the cable was left open. The capacitor of the cable is calculated through reading the measured reactance $(X)$ by means of MiniVNA using equation (5.7): 


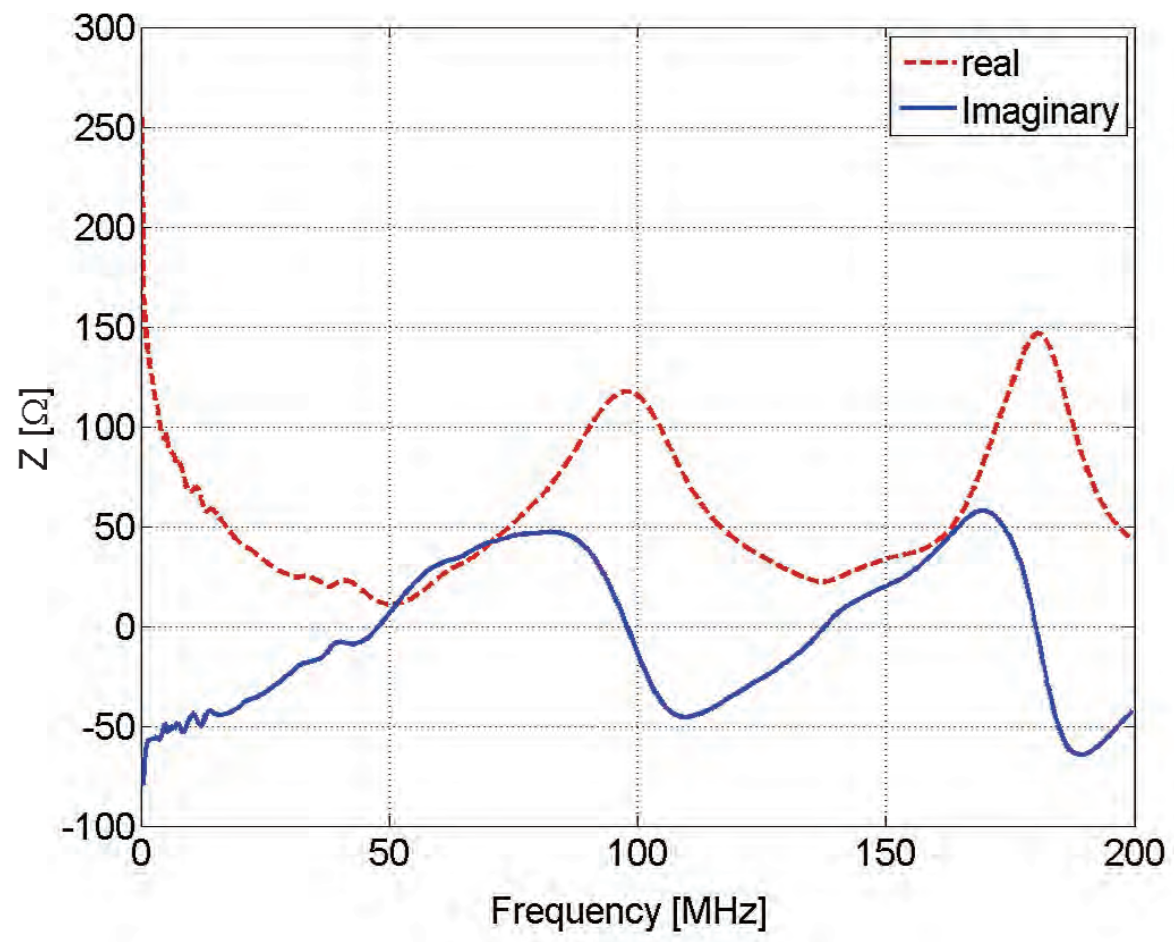

Figure 5.9: The empirical measurement results to determine the electrode-skin impedance. $\mathrm{Z}$ is the combination of electrode-skin and input impedance of the body $\left(Z_{i}\right)$.

$$
X=\frac{1}{2 \pi f C}
$$

where $f$ represents the frequency of the reactance and $C$ is the capacitor of the cable with 1 meter length. According to the resonance frequency $\left(f_{\text {res }}\right)$ achieved from IBC empirical measurements (see figure 5.6), the value of the inductance could be obtained using:

$$
f_{\text {res }}=\frac{1}{2 \pi \sqrt{L C}}
$$

where $\mathrm{C}$ has been calculated by (5.8). Therefore, the components of $R L C$ circuits have the values of $110 \Omega, 51 \mathrm{nH}, 70 \mathrm{pF}$ which representing the resonance frequencies of $90 \mathrm{MHz}$. Finally, by considering the calculated $Z_{i}$ and the model of cable in the 


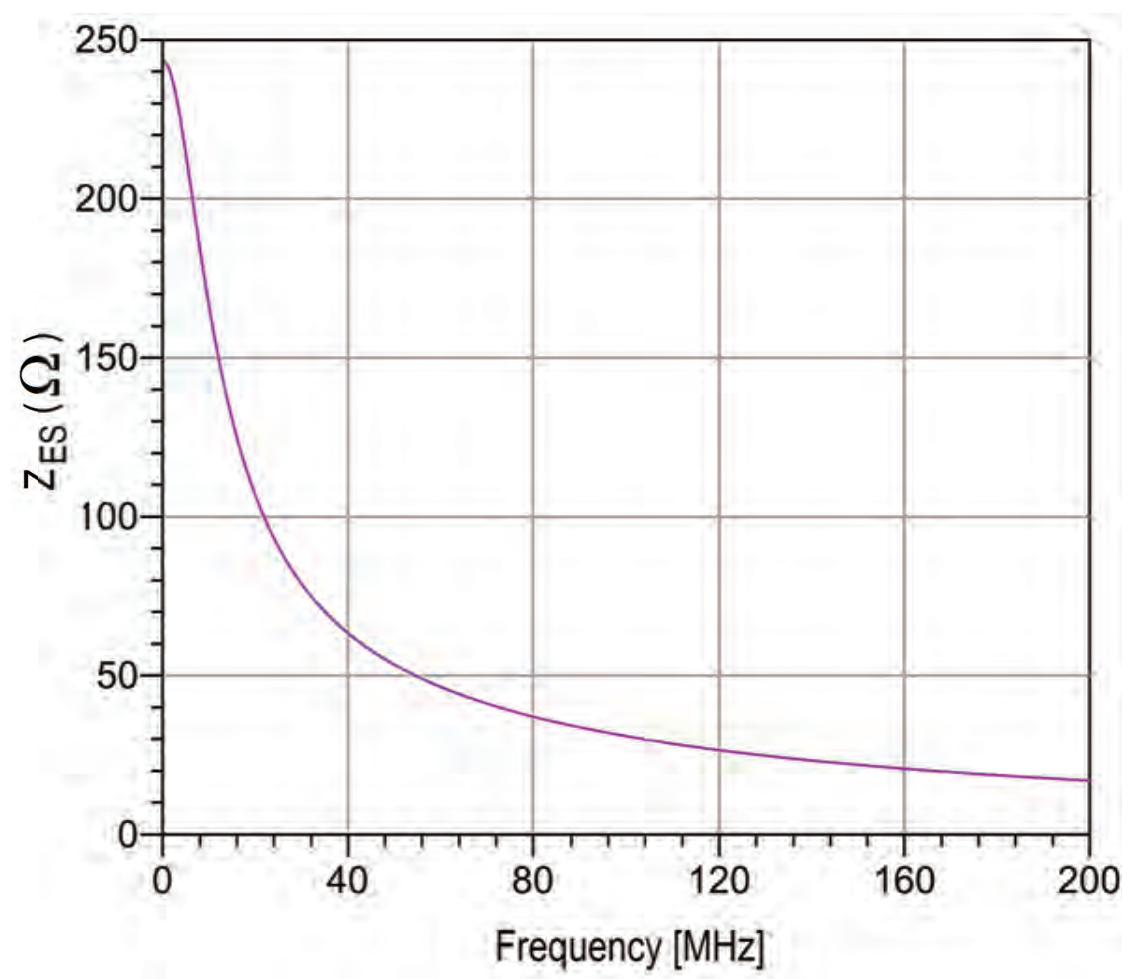

Figure 5.10: The measurement results of electrode-skin impedance.

simulation model, value of $Z_{E S}$ over the desired frequency range in this study is presented in figure 5.10.

Since the air capacitances between on-body electrodes or the connecting cables (in figure A.1) are two small (0.347 fF in our study), they have little effect on the channel propagation, particularly at lower frequencies. Figure 5.11 compares the body path (path 1) and the air path (path 2) impedances. Since the air path has higher impedance compared to the body path in the $20 \mathrm{~cm}$ channel length, it is not included in the proposed models in figure 5.7. 


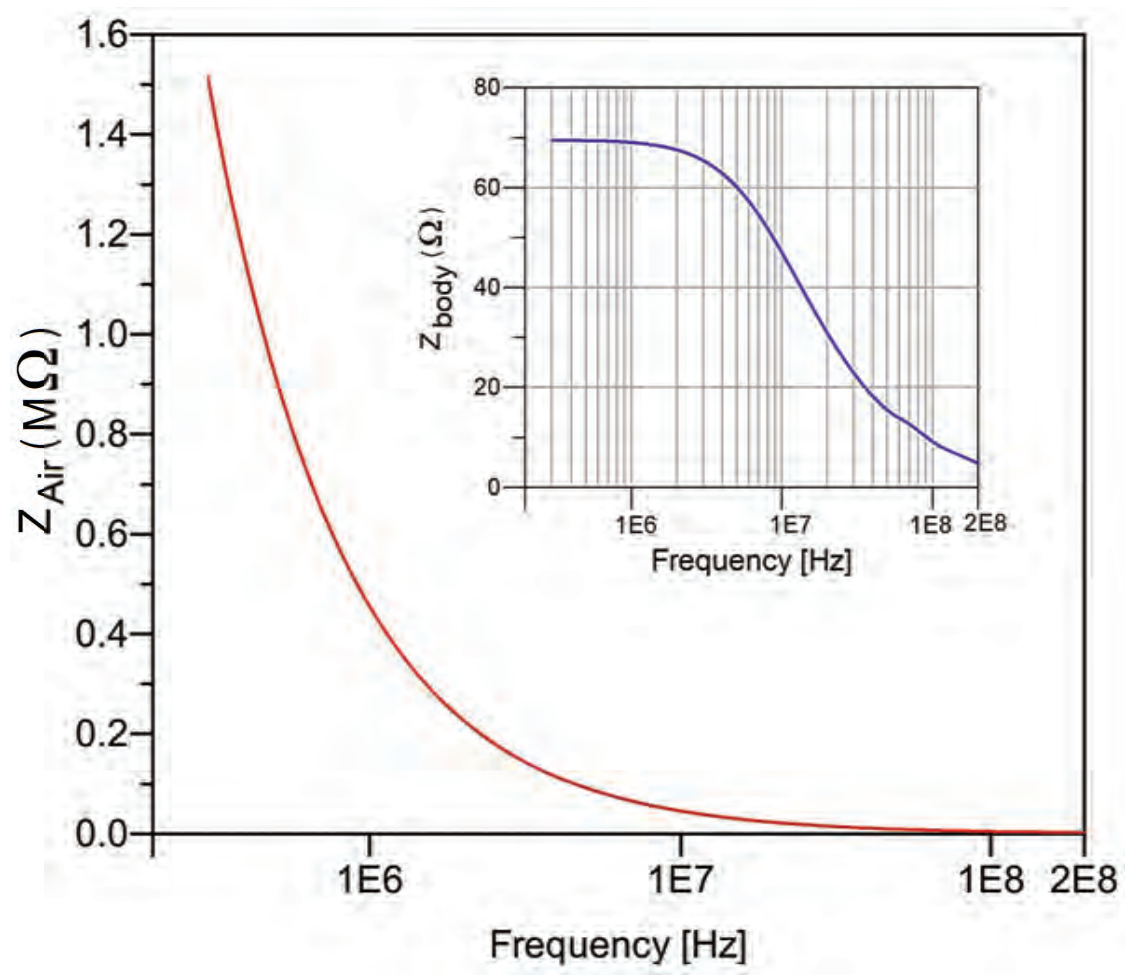

Figure 5.11: The comparison of the air path and the body path impedances between the transmitter and receiver electrodes.

\subsection{Results}

In figure 5.12, both the empirical and simulation results of the IBC signal attenuation are compared. It is worth noticing that, the effects of cables and baluns were not considered in the simulation results of figure 5.12. Moreover, since the empirical results of all subjects show approximately similar behavior (see figure 5.6), the arm layers thickness and geometries of the Male subject \#1 is considered in our simulation (see Table 5.1 and 5.2). The achieved results from simulation indicate higher signal attenuation when the joint was in the transmission path. This change is approximately 3.0 $\mathrm{dB}$ for frequencies below $20 \mathrm{MHz}$ while less than $2.0 \mathrm{~dB}$ variations is observed above 


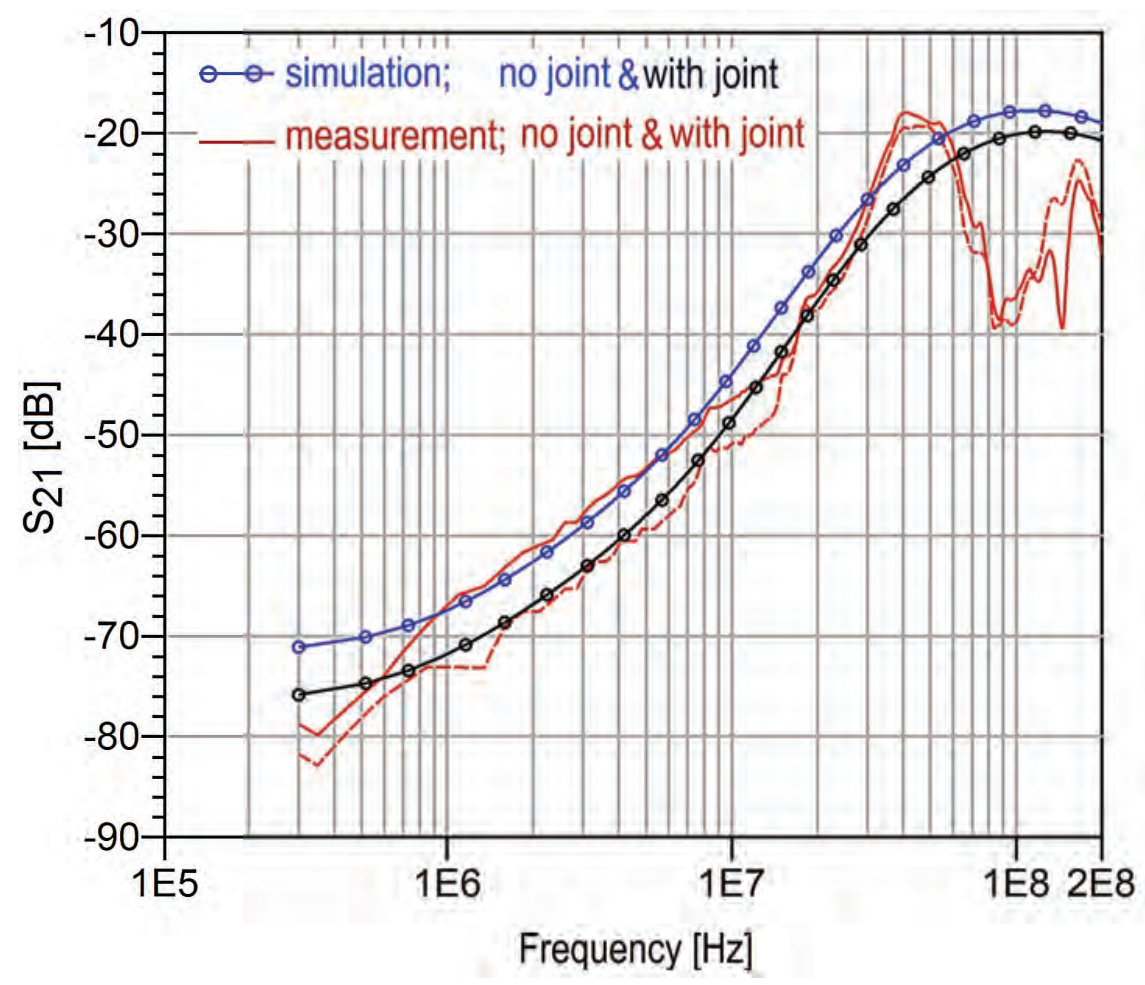

Figure 5.12: Measurement and model simulation (without cables and baluns model) results on human arm as a transmission path.

$20 \mathrm{MHz}$. For instance, at frequency of $5.0 \mathrm{MHz}$, the attenuation is $55.19 \mathrm{~dB}$ and $58.17 \mathrm{~dB}$ based on the simulation results. Meanwhile, good agreements were found between simulation and measurement results only below $54 \mathrm{MHz}$ for both joint and no joint situation.

Figure 5.13 represents the results of both empirical and simulation methods (i.e., circuit model B). Here, the empirical results support the validity of our circuit model (B) over the whole desired frequency range $(0.3-200 \mathrm{MHz})$. In order to assess the proposed circuit models performance (accuracy) at suggested frequency range, the root mean square error (RMSE) between empirical measurements and simulation models were calculated. Figure 5.14 shows the RMSE level of $0.25 \%$ below $20 \mathrm{MHz}$ 


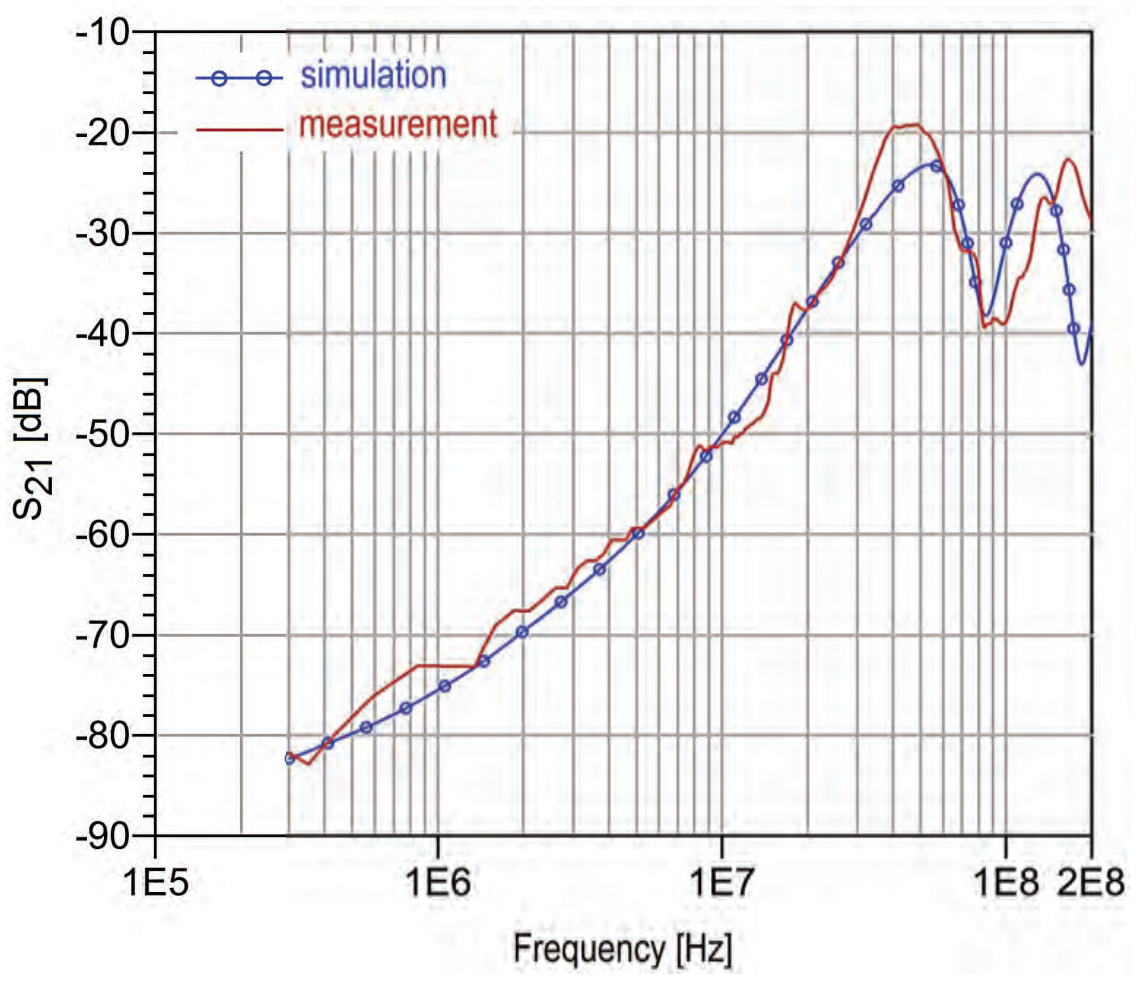

Figure 5.13: The comparison of the empirical and model B simulation results. The effects of cables and baluns were considered in the simulation.

for both circuit models. The highest RMSE of $5.82 \%$ was achieved for model A at $100 \mathrm{MHz}$ while model B showed a highest RMSE of $4.3 \%$ at $180 \mathrm{MHz}$.

The curves in figure 5.15 show the simulation results considering physiological parameters and the geometries of subject's joint when the distance between transmitter and receiver electrodes was $20 \mathrm{~cm}$. It is worth noting that since determining the exact length of the elbow joint is only possible through a medical imaging technique such as magnetic resonance imaging (MRI) scan, the joints lengths were assumed to be 8 , $10,12,14 \mathrm{~cm}$ in this study [93]. The graph shows that increasing joint length causes higher signal attenuation below $60 \mathrm{MHz}$. For instance, at frequency of $10 \mathrm{MHz}$, the attenuations are $50.01 \mathrm{~dB}, 51.35 \mathrm{~dB}, 52.10 \mathrm{~dB}$, and $53.57 \mathrm{~dB}$ for different elbow joint 


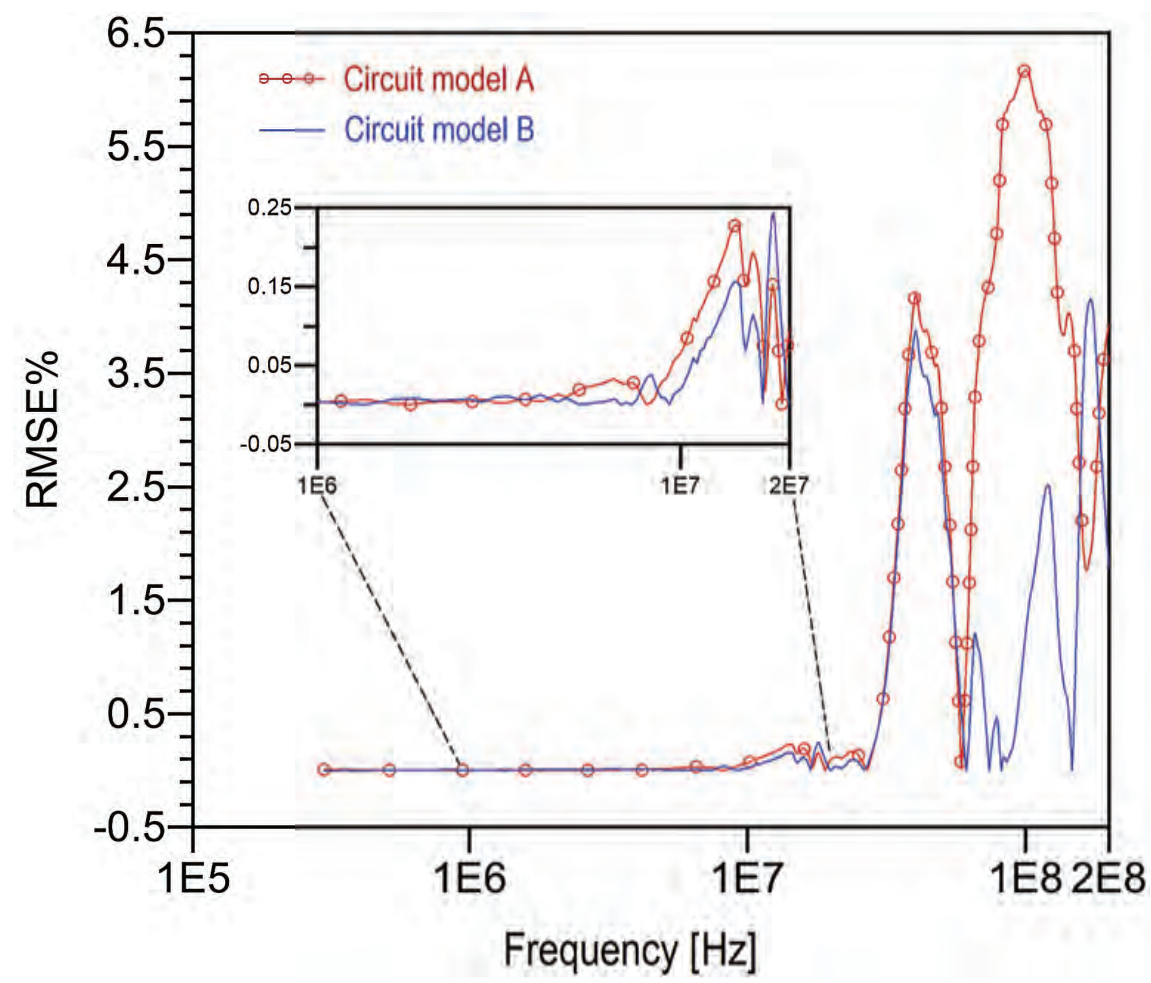

Figure 5.14: The RMSE between empirical and simulation for both proposed models.

lengths of $8.0,10,12,14 \mathrm{~cm}$, respectively. This effect is more prominent at the frequency range of $0.3-20 \mathrm{MHz}$. For instance, at frequency of $300 \mathrm{kHz}$, the difference of signal attenuations is $3.69 \mathrm{~dB}$ when the joint length increases from 8 to $14 \mathrm{~cm}$ while this difference reached $1.77 \mathrm{~dB}$ at $54 \mathrm{MHz}$. However, the variation of joint length $\left(L_{j}\right)$ shows a negligible effect above $60 \mathrm{MHz}$, suggesting that signal propagation path is now not only confined to body tissue.

\subsection{Discussion}

Joint-segments of the human body are necessary for the wide range of motion we currently enjoy [30]. In this chapter, we have presented a new circuit model of the 


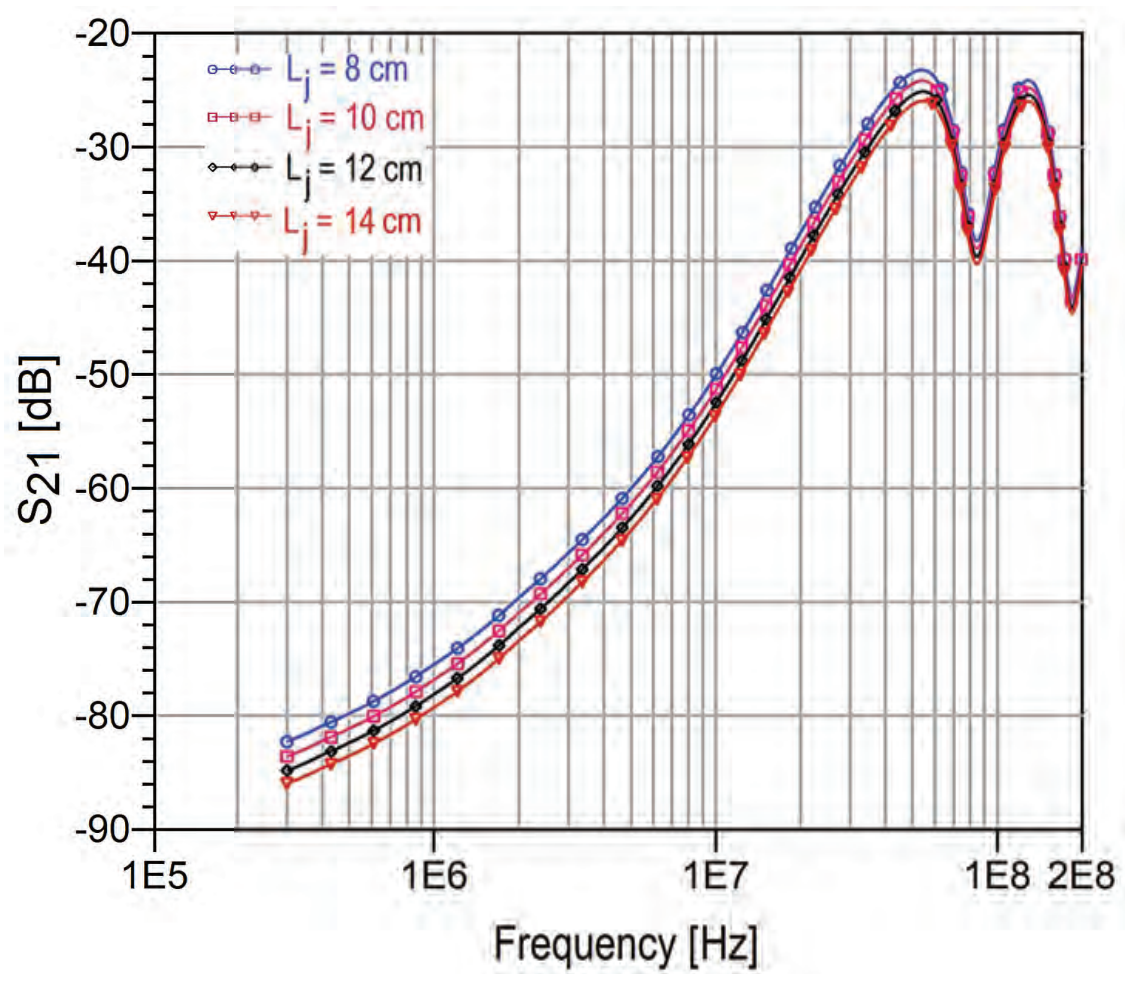

Figure 5.15: Signal attenuation at different elbow joint length $\left(L_{j}\right)$.

human arm taking into account elbow joint effects and the measurement equipment within a larger frequency range of $0.3-200 \mathrm{MHz}$.

Based on the frequency range, the attenuation curves in figure 5.6 can be divided into three regions. First, between $0.3-20 \mathrm{MHz}$ where the effect of joint-segment is most pronounced on the signal attenuation compared to the no joint position. This is thought to be due to the penetration of the current into deepest layer (i.e. bone) when propagated between transmitter and receiver electrodes (path 1 in figure A.1). In between 20 to $54 \mathrm{MHz}$, presence of the joint-segment has a smaller effect $(<1.0 \mathrm{~dB})$ on the signal attenuation. Since increasing the operation frequency can strengthen the surface wave propagation [62], the current communication confines only to the 
skin layer. This could be explained by the air capacitance (see figure A.1) developed between the nearest electrode to ground and the earth ground which becomes a more significant return path through the ground (lower impedance above $20 \mathrm{MHz}$ compared to body tissue) and consequently improves the signal attenuation at this frequency range. At frequencies above $54 \mathrm{MHz}$, the higher intensity of electric field [41] led to the signal transmission through spatial path. Further, the wavelength of body skin above $54 \mathrm{MHz}$ approximately equals to the twice of the body channel length. Thus, the human body acts as an antenna and radiates the IBC signal [24]. For instance, the wavelength of a wet skin is $0.486 \mathrm{~m}$ at $54 \mathrm{MHz}$ which is close to the twice of our body channel length, that is $0.4 \mathrm{~cm}$ in this study. It should be noted that any increment of frequency range reduces the penetration depth of the transmitted signal into the tissues [94]. Therefore, the IBC input signals tend to be radiated rather than penetrating into the body tissues at high frequencies.

Figure 5.12 shows a reasonable agreement between empirical and simulation results within 1-54 MHz for both joint and no joint positions. The differences below 1.0 $\mathrm{MHz}$ could be explained by the presence of baluns. The balun insertion loss (1.0 to $2.1 \mathrm{~dB}$ loss for each balun [91]) needs to be considered in the circuit model. Above 54 $\mathrm{MHz}$, empirical and simulation results show larger differences. The variation of attenuation in higher frequencies could be attributed to the antenna effects of connecting cables and the body.

Both empirical experiments and model simulation results show a progressive decrease in signal attenuation with and without elbow joint in the communication path. As mentioned earlier, at low frequencies (less than $54 \mathrm{MHz}$ ), the variation of attenuation as well as the rate of variation are dependent on signal penetration across different 
tissue layers. This can be explained by the tissue layers possessing differing dielectric properties. For instance, muscle on average has better conductivity of $0.512 \mathrm{~S} / \mathrm{m}$ compared to fat $(0.027 \mathrm{~S} / \mathrm{m})$ within the $0.3-200 \mathrm{MHz}$ frequency range. Hence, greater penetration of signal is expected for muscle. Further, the joint-segment with a large mass of bone marrow and cartilage has even lower conductivity compared to muscle (0.007 S/m and $0.278 \mathrm{~S} / \mathrm{m}$, respectively compared to $0.512 \mathrm{~S} / \mathrm{m}[94])$. Therefore, the presence of joint-segments causes $1.0 \mathrm{~dB}$ to $5.2 \mathrm{~dB}$ higher attenuation between $0.3-20$ MHz. Figure 5.13 shows the results of both empirical and simulation (circuit model B). The signal attenuation trends for the high frequency region (54-200 MHz) were observed when the cables and baluns models were added to the body circuit model A.

We plotted the RMSE\% for both empirical and circuit models in figure 5.14. The comparison between results from model A and B prove the current propagation hypothesis from path 1 and 2 (see figure A.1). A good correlation is observed at frequency range 0.3-54 MHz. Hence, the presence of cables and baluns has negligible effects on signal propagation and the primary signal path is through the body tissue layers. The larger RMSE\% of model B compared to model A 54-200 MHz. indicated that the influence of cables and baluns should be considered at higher frequencies. This is due to the measurement equipment behaves as an antenna at this frequency band and consequently, the signal is primarily either radiated or capacitively coupled through the air (path 2 in figure A.1).

Since the length of joint is different for every individual person, we investigated the effect of four different elbow joint lengths on signal attenuation. Figure 5.15 shows the signal attenuation at different elbow joint length. It can be seen that a $6.0 \mathrm{~cm}$ increment in joint length led to at most 3.69 and $2.78 \mathrm{~dB}$ higher attenuation within the 
frequency bands of $0.3-20$ and $20-60 \mathrm{MHz}$, respectively. This could be due to the large mass of bone in longer joints causing higher signal reflections. Minimal differences in attenuation due to different joint length above $60 \mathrm{MHz}$, again, prove the nonsignificant influence of body tissues in the signal propagation at higher frequencies.

In short, the presence of joints in the signal pathway in addition to joint length should be considered since the IBC method will be affected by the body composition of the user, particularly at frequencies below $54 \mathrm{MHz}$. It should be noted that the mentioned frequency band covers the center frequency for IEEE 802.15.6 HBC standard which is $21 \mathrm{MHz}$. At higher frequencies (above $54 \mathrm{MHz}$ ) the antenna effects of measurement equipment were observed but could still be predicted accurately by model B. Our proposed models offer unique advantages of being more accurate even for high frequency ranges up to $200 \mathrm{MHz}$ compared to previous IBC model. The models also possess the capability to predict the IBC signal attenuation in presence of limb joint. In addition, the effects of measurement equipment were considered in our models which were rarely addressed in past studies. Table 5.3, summarizes the characteristics of our models and compares them to previous IBC models.

These proposed models will aid in the development of an efficient posture-related transceiver based on the IBC technique in the presence of movement for improved intrabody communications.

\subsection{Summary and Conclusion}

IBC is a novel data communication technique using the human body itself as the transmission medium or communication channel. The present study proposed a new 
Table 5.3: The Proposed IBC Models Characteristics Compared to Current Models

\begin{tabular}{|c|c|c|c|c|c|}
\hline $\begin{array}{c}\text { Model } \\
\text { charact- } \\
\text { eristics }\end{array}$ & {$[54]$} & {$[46]$} & {$[57]$} & {$[62]$} & This work \\
\hline \hline $\begin{array}{c}\text { Type } \\
\text { of } \\
\text { model } \\
\text { model } \\
\text { impeda- } \\
\text { nces }\end{array}$ & $\begin{array}{c}\text { Circuit } \\
\text { impedances })\end{array}$ & $\begin{array}{c}\text { Circuit } \\
\text { impedances })\end{array}$ & $\begin{array}{c}\text { Circuit } \\
\text { model }(10\end{array}$ & $\begin{array}{c}\text { Theoretical } \\
\text { model }\end{array}$ & $\begin{array}{c}\text { model }(14 \\
\text { impedances } \\
\text { including } \\
\text { limb joint) }\end{array}$ \\
\hline $\begin{array}{c}\text { Frequency } \\
\text { range }\end{array}$ & $\begin{array}{c}1.0 \mathrm{k} \\
\text { to } 1.0 \mathrm{MHz}\end{array}$ & $\begin{array}{c}1.0 \mathrm{k} \\
\text { to } 1.0 \mathrm{MHz}\end{array}$ & $\begin{array}{c}100 \mathrm{k} \\
\text { to } 5.0 \mathrm{MHz}\end{array}$ & $\begin{array}{c}1.0 \mathrm{M} \\
\text { to } 100 \mathrm{MHz}\end{array}$ & $\begin{array}{c}300 \mathrm{k} \\
\text { to 200MHz }\end{array}$ \\
\hline $\begin{array}{c}\text { Electrode } \\
\text { configur- } \\
\text { ation }\end{array}$ & $\begin{array}{c}\text { Two- } \\
\text { and Four- } \\
\text { electrode }\end{array}$ & $\begin{array}{c}\text { Four- } \\
\text { electrode }\end{array}$ & $\begin{array}{c}\text { Four- } \\
\text { electrode }\end{array}$ & $\begin{array}{c}\text { Two- } \\
\text { electrode }\end{array}$ & $\begin{array}{c}\text { Four- } \\
\text { electrode }\end{array}$ \\
\hline $\begin{array}{c}\text { IBC } \\
\text { coupling } \\
\text { method }\end{array}$ & $\begin{array}{c}\text { Capacitive } \\
\text { and }\end{array}$ & $\begin{array}{c}\text { Galvanic } \\
\text { Galvanic }\end{array}$ & $\begin{array}{c}\text { Galvanic } \\
\text { Capacitive } \\
\text { and } \\
\text { body surface } \\
\text { propagation }\end{array}$ & $\begin{array}{c}\text { General } \\
\text { IBC model }\end{array}$ \\
\hline $\begin{array}{c}\text { Error } \\
\text { analysis }\end{array}$ & $\begin{array}{c}\text { Not } \\
\text { reported }\end{array}$ & $\begin{array}{c}\text { Not } \\
\text { reported }\end{array}$ & $\begin{array}{c}2.0 \mathrm{~dB} \\
\text { absolute } \\
\text { error }\end{array}$ & $\begin{array}{c}\text { Not } \\
\text { reported }\end{array}$ & $\begin{array}{c}\text { RMSE } \\
<5.82 \%\end{array}$ \\
\hline
\end{tabular}

body channel model and focused on signal attenuation in IBC systems due to limbjoint effects. The joint-segment presence effect on signal transmission path was investigated in this work. Based on both measurements and simulations results, it was concluded that the increase in attenuation was proportional to the joint length between the forearm and upper arm at the low frequency range. It was also found that measurement equipment behave as an antenna at higher frequencies (above $54 \mathrm{MHz}$ ). Simulation results indicated that the constant increasing of a joint length leads to increasing the attenuation by an approximately constant amount. Results of both human experiments and model simulation reveal that the presence of a joint between the signal transmission pathway causes an additional 1.0 to $5.2 \mathrm{~dB}$ attenuation. The results are from different test subjects at frequency ranges of $0.3-54 \mathrm{MHz}$ for path 
lengths of $20 \mathrm{~cm}$. Below $20 \mathrm{MHz}$, the models provide more accuracy with a percentage root mean square error (RMSE) of only $0.25 \%$. In addition, a longer length of the elbow joint increases the signal attenuation with an approximately linear rate of 0.6 $\mathrm{dB} / \mathrm{cm}$, below $20 \mathrm{MHz}$. The results suggest that the signal is propagates through the body tissues below $54 \mathrm{MHz}$ when the effects of measurement equipment e.g., cables are negligible. 


\section{Chapter 6}

\section{IBC System Design}

The significance of developing a human-centric WBAN system lies in the vital role that it plays in transmitting human physiological parameters to enable long-term healthcare monitoring. This raises research and development issues concerning transceiver circuit design, particularly reducing power consumption while improving the data rate. Among the WBAN physical layers, IBC is emerging as an energy efficient technique compared to both UWB and NB. For instance, while the reported energy consumption for an UWB communication system was $2.5 \mathrm{~nJ} / \mathrm{b}$ [95], the latest IBC system consumed only $0.24 \mathrm{~nJ} / \mathrm{b}[21]$. In comparison, the recently reported IEEE 802.15.6 NB RF transceiver chip consumes 7.9 nJ/b [96]. Therefore, IBC appears to be more energy efficient and enticing for WBAN medical applications compared to UWB and NB PHYs.

In this chapter, we present a novel energy efficient IBC system employing a digital baseband signaling technique. The measurement and modeling results in past chapters indicate that the four-electrode IBC configuration, which is called galvanic coupling below $5 \mathrm{MHz}$ [57], is a more reliable approach compared to two-electrode 
configuration. Therefore, we focus on developing the galvanic coupling IBC system with the aim of improving data throughput. A carrier-free transmission is implemented in a FPGA board based on a pulse position modulation (PPM). PPM is a modulation scheme which uses time-based pulse positioning to encode data and is widely used in radio frequency impulse radio (IR) propagations. In this chapter, we propose the galvanic coupling IBC based on IR for the first time and refer to it as IR-IBC. The chapter is organized as follows: Section 6.1 explains a brief background of the past implemented IBC systems. Section 6.2 elaborates the details of using a square wave as the excitation test signal to measure the IBC channel's frequency response. Then, our proposed IBC design and implementation on the FPGA board and analog front end is examined in section 6.3. The final issue to be addressed is to present a summary of what has been discussed about IBC system so as to provide the reader with an overall picture of the design and implementation of IBC physical layer.

\subsection{IBC Hardware Overview}

A typical bidirectional IBC transceiver essentially consists of three fundamental components in the same package including a transmitter, a receiver, and a digital control unit (microcontroller) as shown in figure 6.1. A digital control unit is required to control the transceiver by sending or receiving messages based on a defined protocol. The transmitter and the receiver may include several sub-systems such as an "analog-todigital" or "digital-to-analog" convertor (ADC or DAC), a channel coding technique, and particular modulation or demodulation scheme. The decision to add each of the mentioned sub-systems determines characteristics of the transceiver system such 


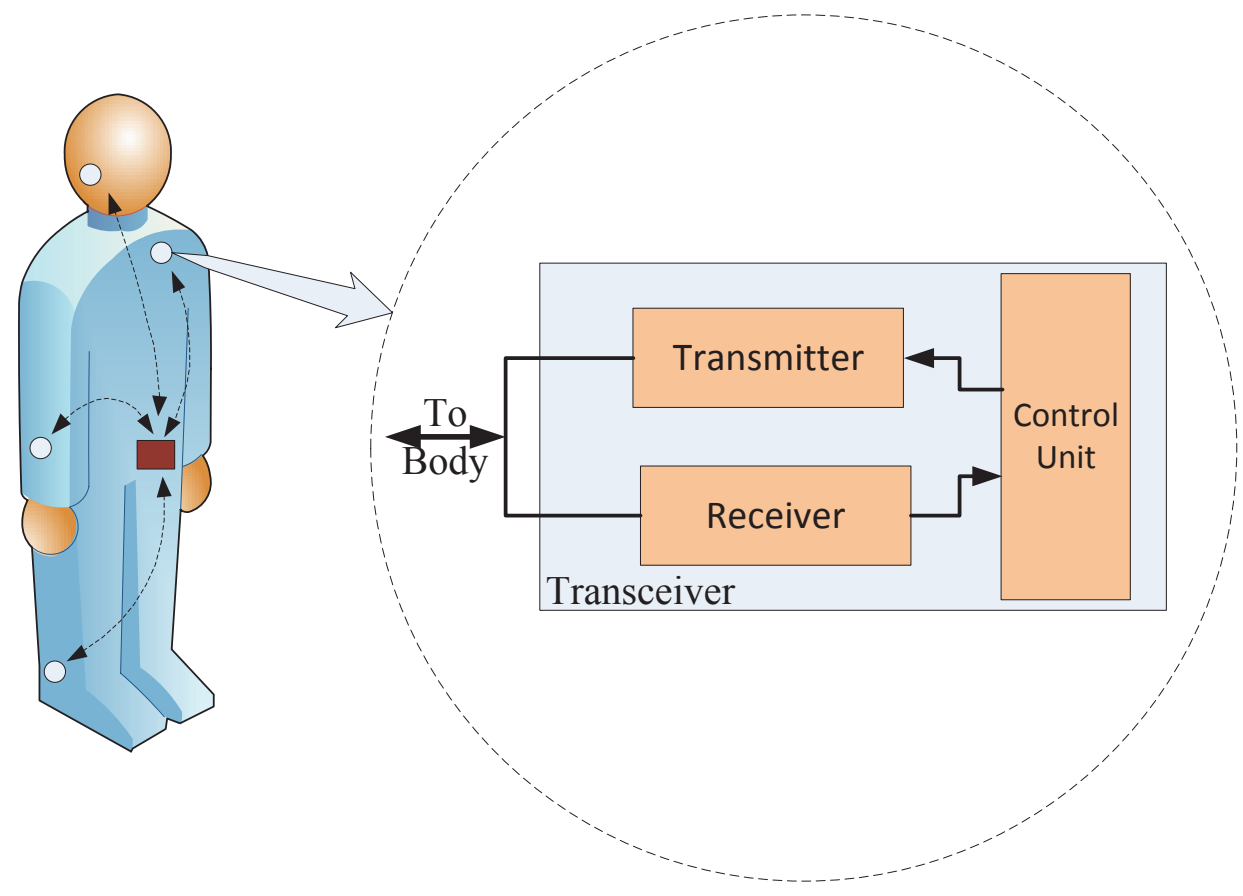

Figure 6.1: Bidirectional IBC Transceiver.

as data rate, power consumption, energy efficiency, and operation frequency range. Based on different types of biomedical signals, such as analog or digital, the IBC transceiver may consist of one or more subsystems such as ADC or DAC [97]. Also, due to the human body channel limitations and the body tissue characteristics in different frequency domains, direct data transmission through the human body could lead to a high transmission voltage or power [67]. This suggests that the signals produced by various medical devices are not always suitable for direct transmission over a body channel. Hence, a coding technique or a modulator-demodulator scheme should be considered in IBC system design to influence the complexity, energy and cost efficiency of the IBC system.

In some of the past studies, the coded digital signal was directly transmitted 
through the body without using any modulation schemes. For instance, Harikomar et al. [98] employed a digital Manchester coding technique for IBC system. Since neither modulation module nor $\mathrm{ADC}$ is required in the digital IBCs, their system demonstrated a power consumption of $2.0 \mathrm{~mW}$. However, an error in Manchester code signals leads to a large bit error in the entire received signal.

The fundamental digital modulation schemes such as the amplitude-shift keying (ASK), frequency-shift keying (FSK), and phase-shift keying (PSK) have been deployed in IBC systems [16]. These low-speed narrow-band modulation schemes need to operate in a high frequency range to carry the digital signal which causes the whole system to have high power consumption. However, a recent double-FSK modulation scheme on the capacitive coupling IBC transceiver indicated a reasonable transceiver power consumption of $5.4 \mathrm{~mW}$ with data throughput up to $10 \mathrm{Mbps}$ [21].

The maximum IBC data rate is reported as $1312.5 \mathrm{kbps}$ in the $21 \mathrm{MHz}$ frequency band by IEEE 802.15.6 standard [17]. Although, this data rate is suitable for medical data transmission, e.g. capsule endoscope and glucose monitoring, some healthcare applications such as medical imaging might require higher data rates above $10 \mathrm{Mbps}$ [21]. Recently, a modified frequency selective digital transmission (MFSDT) was employed in the IBC transmitter to provide thrice the data rate (3.9375 Mbps) [25]. In the proposed work by KAIST (Korea Advanced Institute of Science and Technology) research group, a new WBAN transceiver chip for multichannel electro-acupuncture application was proposed [99]. The carrier frequency of the system was $21 \mathrm{MHz}$ with a scalable data rate of $1.3125 \mathrm{Mbps}$ and the power dissipation of $5.5 \mathrm{~mW}$ when the receiver was activated. However, this study did not propose any method to enhance data throughput of WBAN.

In further experiments on the galvanic coupling IBC by Wegmueller [9], a battery 
powered transmitter and receiver was implemented in frequency range of $10 \mathrm{kHz}$ to 1.0 MHz. The proposed system was composed of an analog sub-circuit which led to high power consumption of $726 \mathrm{~mW}$ with a maximum $64 \mathrm{kbps}$ data rate. Galvanic coupled transceivers appear to require more power potentially due to the higher attenuation in both signal forward and return path through the body. Hence, it has not as yet been reported to fulfill the WBAN standard requirements. Thus, a new IBC system design is required to improve the data throughput and power consumption of galvanic coupling. The signal propagation in galvanic coupling tacks place within the human tissue and the body tends to screen out external interfering signals. Therefore, the galvanic coupling is the suitable IBC method for future WBAN applications.

The main objective of this chapter is to design and implement a novel energy efficient transmitter and receiver, i.e. high data throughput as well as low power consumption architecture for IBC applications. Since galvanic coupling method is less prone to the environmental effects as seen in capacitive one (see chapter 4), it has been selected for our proposed system in the study. However, the reduced bandwidth of the galvanic method (less than $5.0 \mathrm{MHz}$ [57]) compared to the capacitive one (up to $100 \mathrm{MHz}[27])$ causes a lower data throughput. Therefore, with the aim to improve a data throughput of the galvanic IBC, we develop a carrier-free scheme based on impulse radio (IR) which is implemented on FPGA in this chapter. Also, in-vivo experiments are conducted to evaluate the communication schemes for input digital signal within the galvanic coupling IBC. 


\subsection{High Frequency Pulse Transmission}

To design and implement an appropriate digital IBC system, we first need to investigate an empirical observation which explores square wave propagation through the human body. In this work, the pulse wave was transmitted through the body in different frequencies while the amplitude was constant. The attenuation or channel loss was calculated for each frequency point using (6.1):

$$
S_{21}=20 \log _{10}\left(\frac{V_{\text {receiver }}}{V_{\text {transmitter }}}\right)
$$

where $V_{\text {transmitter }}$ and $V_{\text {receiver }}$ depicts the amplitude of the transmitted and the received signal, respectively.

Figure 6.2 shows the measurement setup used in the experiments. In this section, rather than using a MiniVNA Pro, a data timing pulse generator (DTG 5274, Tektronix Inc., OR, USA) and a mixed signal oscilloscope (MSO 5204, Tektronix Inc., OR, USA) were employed as the transmitter and receiver, respectively. A pair of transmitter electrodes was employed as an interface to transmit pulse waves through the body arm. Two electromyography (EMG) snap leads, with $115 \mathrm{~cm}$ length, were used as connecting cables between on-body electrodes and baluns. The baluns were directly connected to the transmitter and receiver. To minimize the effects of the cable movements on measurement results, neoprene straps (with Velcro) were used to attach cables to the arm. Meanwhile, a coaxial cable was connected directly from the pulse generator to the oscilloscope as a reference.

The pulse generator provided a waveform with amplitude of $1.0 \mathrm{~V}$ and a $50 \%$ duty cycle at different frequencies between $0.3-200 \mathrm{MHz}$ band. Figure 6.3 shows a $50 \mathrm{MHz}$ pulse wave which was generated by the pulse generator to transmit into the 


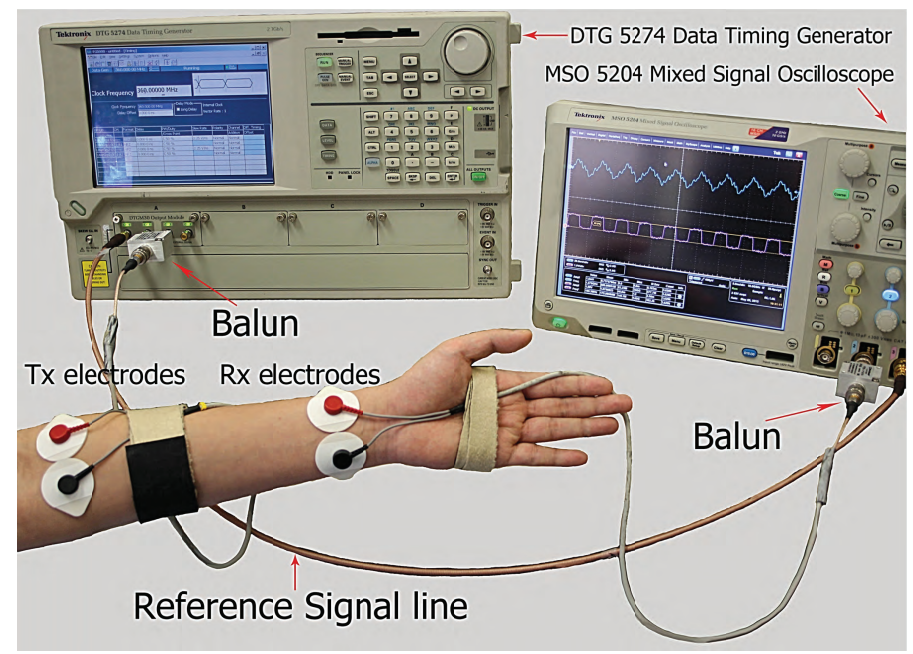

Figure 6.2: The measurement setup of the IBC technique.

IBC channel.

Healthy subjects, one male and one female, volunteered to participate in this part of our research study. The subjects were: 27 and 30 years old, 162 and 182 $\mathrm{cm}$ height, and 57 and $75 \mathrm{~kg}$ weight for female and male, respectively. The signal generator was set to a low input value of $1.0 \mathrm{~V}$ for safety reasons based on ICNIRP exposure guidelines and recommendations [84]. The measurements for each subject were repeated three times over several days and average data were used in analysis.

In our measurements, same as chapter 4 , receiver electrodes were firstly attached on the subject's left forearm and the transmitter electrodes were positioned on the upper left arm to measure the influence of the joint-segment. The distance between transmitter and receiver electrodes was $20 \mathrm{~cm}$, while the elbow joint was located in the middle (10 $\mathrm{cm}$ from each transmitter and receiver electrodes, respectively). In the next experiment, the electrodes of both transmitter and receiver were attached to subject's left forearm $20 \mathrm{~cm}$ apart, and the receiver electrodes were close to the wrist, to simulate the "no joint" condition on a limb segment of similar length. To 


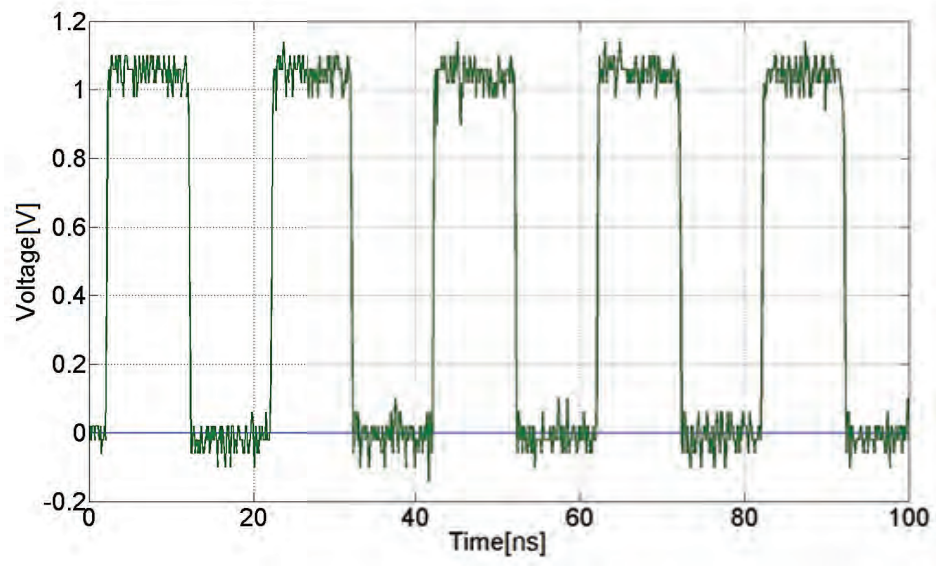

Figure 6.3: Transmitted pulse wave $(50 \mathrm{MHz})$ through the body using IBC method.

minimize any environment interference on the IBC the short distance of $20 \mathrm{~cm}$ between transmitter and receiver has been chosen. During both experimental conditions interelectrode distance was set to $3.8 \mathrm{~cm}$. The inter-electrode distance was the minimum distance between the centers of the electrodes and each electrode had a radius of 1.9 $\mathrm{cm}$ (see chapter 3 for more details).

The subjects were asked to stand and hold their left arm horizontally (parallel with earth ground) while the arm distance from earth ground was 128 and $152 \mathrm{~cm}$ for female and male subject, respectively. At each position, the received signal amplitude was measured by the oscilloscope for a pulse waves with eleven different frequencies within the range of $0.3-200 \mathrm{MHz}$.

The signal attenuation of the communication channel was calculated by the measured signal by means of oscilloscope. Table 6.1 shows the calculated attenuation using equation (6.2) for the male test subject for different frequency points. The yielded signal attenuation was then plotted against the frequency for both "with joint" and "no joint" positions. It is worth noting that, the calculated attenuation is 
Table 6.1: The Characteristics of IBC Received Signal from the Body of Male Subject

\begin{tabular}{|c|c|c|}
\hline $\begin{array}{c}\text { Frequency } \\
\mathrm{MHz}\end{array}$ & $\begin{array}{c}\text { Received signal amplitude }[\mathrm{mV}] \\
\text { Joint/no joint }\end{array}$ & $\begin{array}{c}\text { Attenuation }[\mathrm{dB}] \\
\text { Joint/no joint }\end{array}$ \\
\hline \hline 0.5 & $0.4 / 0.6$ & $68.0 / 64.4$ \\
\hline 1.0 & $0.4 / 0.8$ & $68.0 / 61.9$ \\
\hline 10 & $11.6 / 14.4$ & $38.7 / 36.8$ \\
\hline 20 & $11.2 / 17.2$ & $39.0 / 35.3$ \\
\hline 40 & $35.2 / 43.2$ & $29.1 / 27.3$ \\
\hline 50 & $52.8 / 58.8$ & $25.5 / 24.6$ \\
\hline 60 & $32.0 / 36.2$ & $29.9 / 28.8$ \\
\hline 80 & $10.8 / 9.20$ & $39.3 / 40.7$ \\
\hline 100 & $3.20 / 5.50$ & $49.9 / 45.2$ \\
\hline 150 & $30.0 / 50.0$ & $30.5 / 26.0$ \\
\hline 200 & $3.30 / 8.00$ & $49.6 / 41.9$ \\
\hline
\end{tabular}

the combination of human body path loss $\left(P L_{b o d y}\right)$, the loss of two baluns $\left(P L_{b a l u n}\right)$, and the connecting cables path loss $\left(P L_{\text {cable }}\right)$ :

$$
\text { Attenuation }=P L_{\text {body }}+P L_{\text {balun }}+P L_{\text {balun }}+P L_{\text {cable }}
$$

Figure 6.4 shows the attenuation characteristics for both female and male subject's arm during joint and no joint conditions, respectively. The behavior of attenuation in both the joint and in no joint positions is similar to what we have observed in chapter 4 based on MiniVNA Pro measurements. Therefore, both square waves and sinusoidal waves indicated the similar behavior during communication through the human body at the frequency range of 0.3-200 MHz. It was observed that within the frequency region of 0.3 to $50 \mathrm{MHz}$, the channel loss decreased when the frequency 

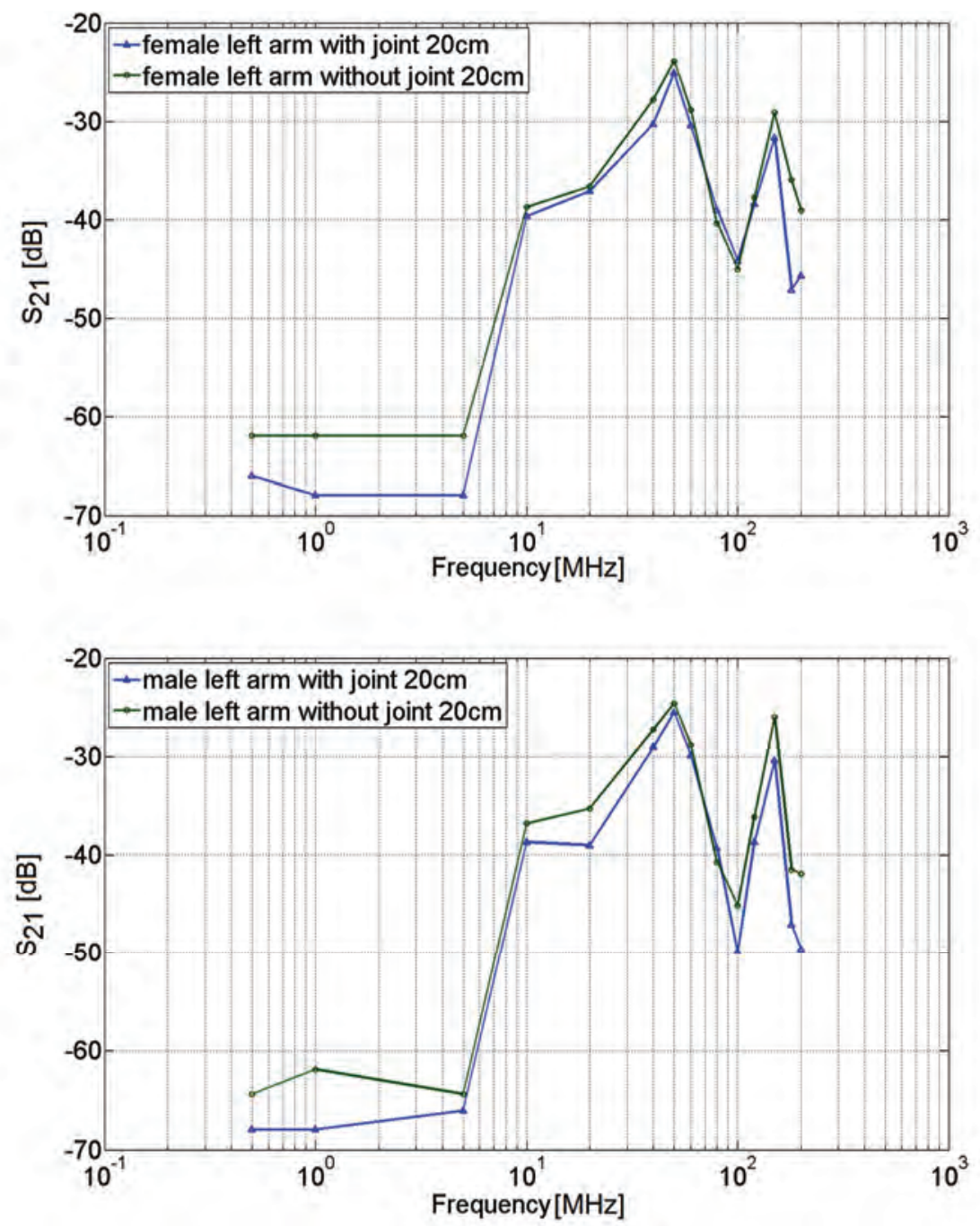

Figure 6.4: The signal attenuation of the arm for 0.3-200 MHz.

increases. This could be explained by the propagation of the signal through the body tissue layers beginning from skin to fat and to muscle at the end. The effect of jointsegments on signal attenuation in IBC appears to be more dominant in this frequency range, as the signal is predominantly coupled through the body tissues. For further details refer to chapter 4 .

The received signal for both test subjects are shown in figure 6.5 and 6.6. The 

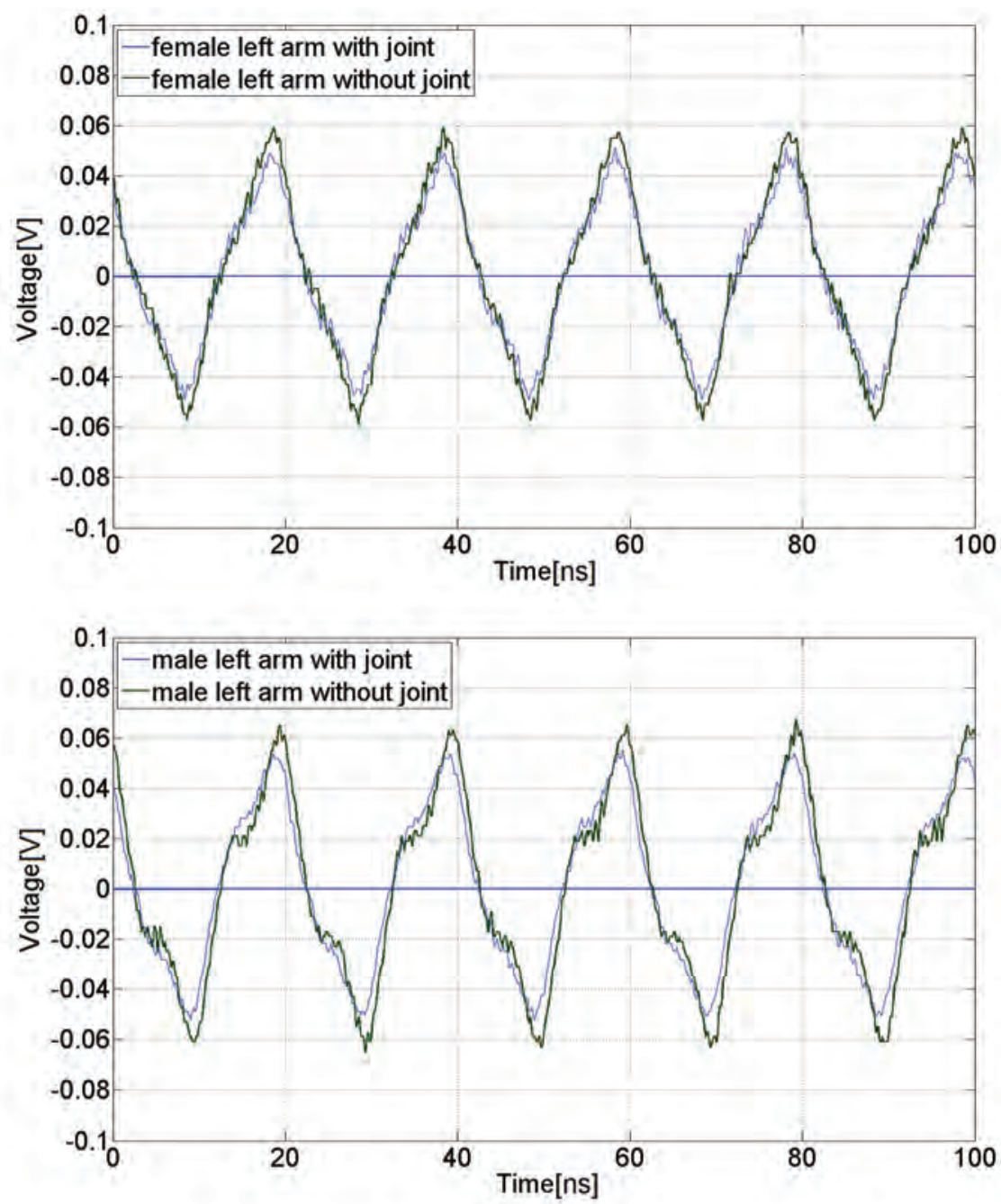

Figure 6.5: IBC received signal in $50 \mathrm{MHz}$ for both female male test subject.

results show that the received signal has lower amplitude when a joint-segment is present among communication channel. For example, the results from female subject's arm (joint-included channel) show that the strength of transmitted signal decreased from $1.0 \mathrm{~V}$ to $118 \mathrm{mV}$ (peak to peak) at $50 \mathrm{MHz}$ frequency. In the same condition, the signal amplitude attenuates to $132 \mathrm{mV}$ for the male subject. In addition, the output voltage difference between joint and no joint positions was less that $12 \mathrm{mV}$ in 

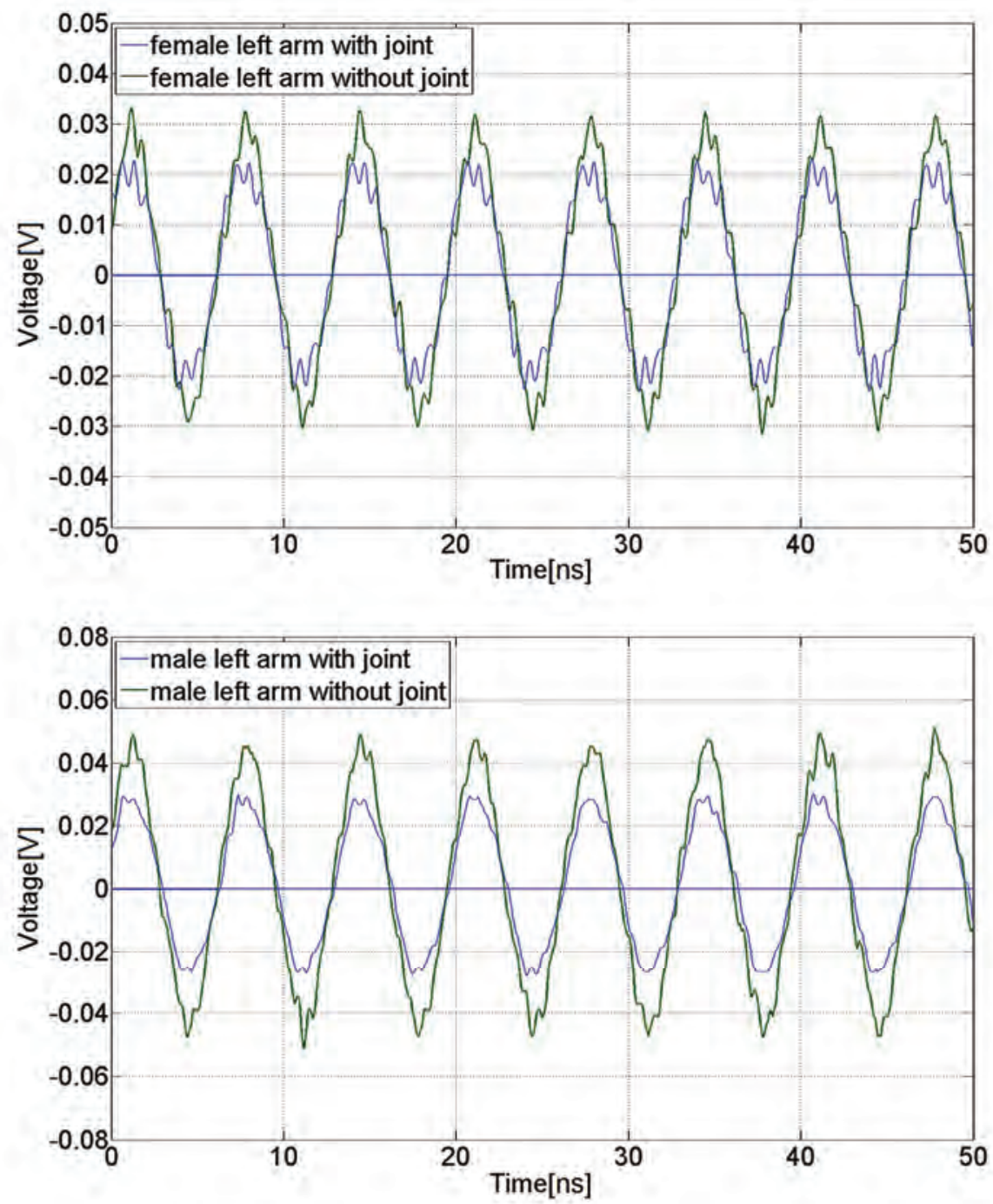

Figure 6.6: IBC received signal in $150 \mathrm{MHz}$ for both female male test subject.

peak points. The received signals are still periodic (see figure 6.5 and 6.6) but do not appear to be digital as per transmitted signals shown in figure 6.3. 


\subsubsection{Pulse Duty Cycle Effect on IBC}

The impact of transmitted pulse duty cycle on IBC is examined through the empirical experiments in this section. Duty cycle represents the proportion of the time duration in which a function is active over the period of the function. The value of duty cycle is usually expressed as a percentage. In this section, we employed similar measurement setup and protocol (see figure 6.2) for examination of duty cycle effects on signal propagation through the human body. During the measurement, we varied the signal frequency to 10, 20, 30, 50 and $60 \mathrm{MHz}$ and adjust the Data Generator to let it output the different duty cycle pulse for transmission through the body. We repeat the IBC experiment in each frequency, and observe the received signal by oscilloscope while recording the duty cycle.

Figure 6.7 demonstrates the attenuation characteristics at the frequency range

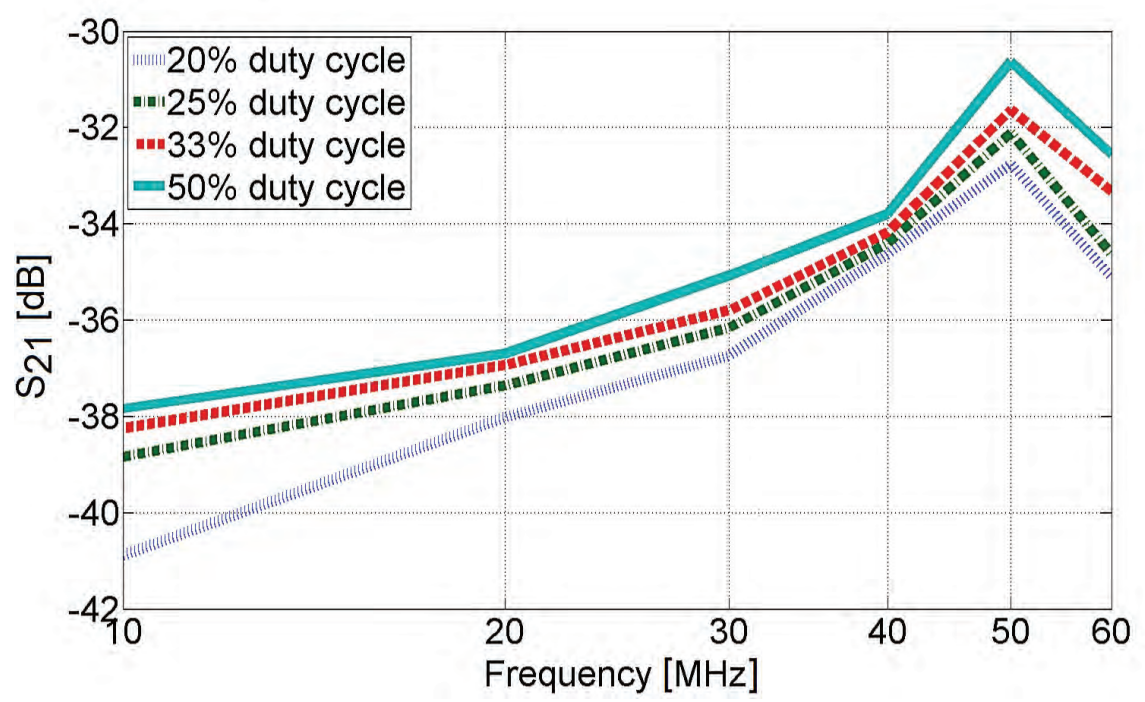

Figure 6.7: The signal attenuation of the body channel for input signal with 20\%-50\% duty cycle when Data Generator was used as transmitter. 
(10-60 MHz) for four different duty cycles of 20\%, 25\%, 33\%, and 50\%. Results show that the signals with lower duty cycle suffer higher attenuation. The duty cycle can be used to simulate user occupancy in a time division multiplexed pulse position based modulation (PPM) scheme. For instance, the duty cycle of signal is $50 \%$ when half of the available positions are occupied by users. This leads to low power spectrum density when the duty cycle of signal is low compared to the signal with higher duty cycle. Therefore, lower body channel attenuation is obtained during higher frequency transmissions as well as higher duty cycles. This suggests that the digital baseband signals deployed in a time division multiplexed mode with higher timeslot occupancy (user occupancy) leads to lower attenuation during the IBC transmissions.

\subsection{IBC Hardware Architecture}

The IBC architecture should be simple, have high data throughput, and be energy efficient. Since the output power of analog transmitter might be varied due to temperature variation as well as high power consumption of analog transmitter, a digital transmitter is proposed in this work. We suggest a carrier-free scheme based on IR for the IBC hardware design. We argue that an optimal modulation scheme for IR-IBC would fulfill the requirements to design a more efficient IBC hardware [100].

Both digital baseband OOK and PPM schemes are commonly employed in the systems based on IR, such as IR-UWB transceiver system design [16]. In terms of the complexity of different modulation schemes, both schemes are the potential candidates that allow the implementation of simple transmission circuit. The channel noise detection is simple in PPM compared to OOK, due to the constant envelope of PPM [100]. Unlike OOK, a threshold to distinguish signals and noise is not required 
in PPM [101]. Moreover, PPM scheme provides opportunity to design an interferencefree receiver without any bulky off-chip filter [102]. Since the body channel indicates no multipath effect neither spreading delay, PPM is more immune to error detection compared to OOK [35]. Therefore, a robust, power efficient [104], and high-speed IR-IBC is developed by employing PPM scheme in this study.

To implement our IBC architecture, a FPGA development board was employed. One of the significant features of FPGAs is that they are able to perform in the wide frequency range up to 500MHz. Other features of FPGAs are achievable through increasing the unprecedented logic density, such as embedded processors, digital signal processing (DSP) blocks, simple design cycle, clocking, and high-speed serial. FPGAs are a compelling proposition for almost any type of design even at lower price [103]. The system based on the FPGA provides advance features such as low power and cost, high flexibility, and high data rate. Therefore, the IBC system was implemented on FPGA instead of ASIC design in this stage. The proposed transmitter and receiver circuits of IBC were built on a Xilinx Virtex5-XC5VLX50T FPGA Genesys board (Digilent Inc., WA, USA). The clock frequency of FPGA board is $100 \mathrm{MHz}$. Also, a 3.3 $\mathrm{V}$ power supply is required to run the IBC system. A battery was used to run the FPGA in order to produce a real IBC situation and to isolate the system from main power effects. Figure 6.8 shows the proposed IR-IBC architecture. It is composed of three different units: a transmitter and receiver units implemented on the FPGA as well as an analog front-end (AFE) unit in the receiver side.

\subsubsection{Transmitter Unit}

To design a digital energy-efficient IBC system, the width of the transmitted pulses should be considered as one of the significant parameters. Therefore, IR as a train of 


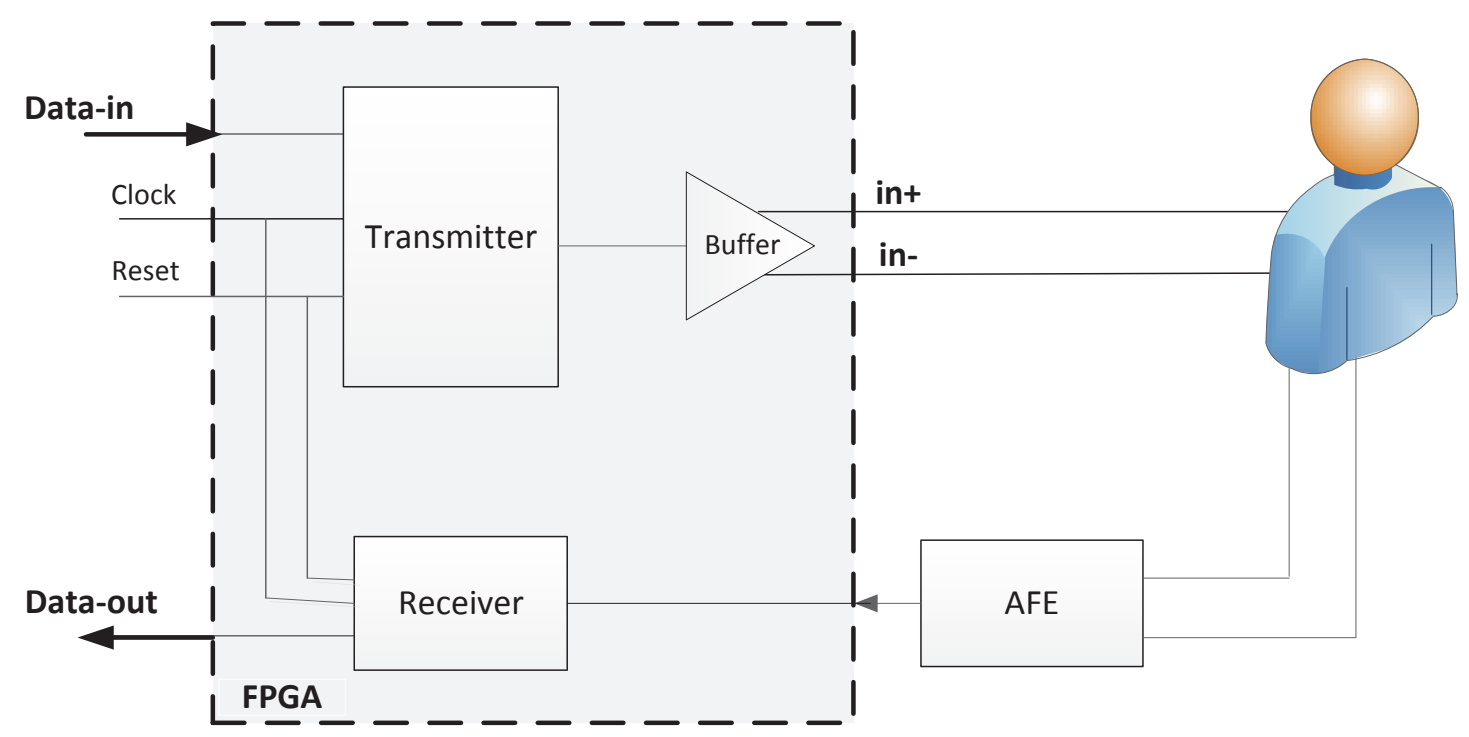

Figure 6.8: The architecture of proposed IBC PPM.

short duration pulses is chosen to propagate through the body channel in our study. IR is able to propagate the signal in wideband range of DC to several gigahertzes. The most wireless sensor networks employ IR-UWB technique [16] due to the inherent wideband nature, low power, and low transmitter hardware complexity.

PPM is a well-known modulation scheme is widely used in IR due to the simplicity of its transmitter and receiver structure and signal detection [105]. It uses pulse positions to encode the original data signal. The PPM causes the variations of pulse polarities over an extended period of time. This breaks the regular intervals in the transmission and smoothen the overall spectrum by diminishing the line spectrum components [104]. Figure 6.9 describes the general structure of the proposed PPM transmitter in IBC method while the output of PPM transmitter is connected to the human body via a differential buffer (see figure 6.8). The buffer is required to create a differential signal for transmission through the body. Additionally, figure 


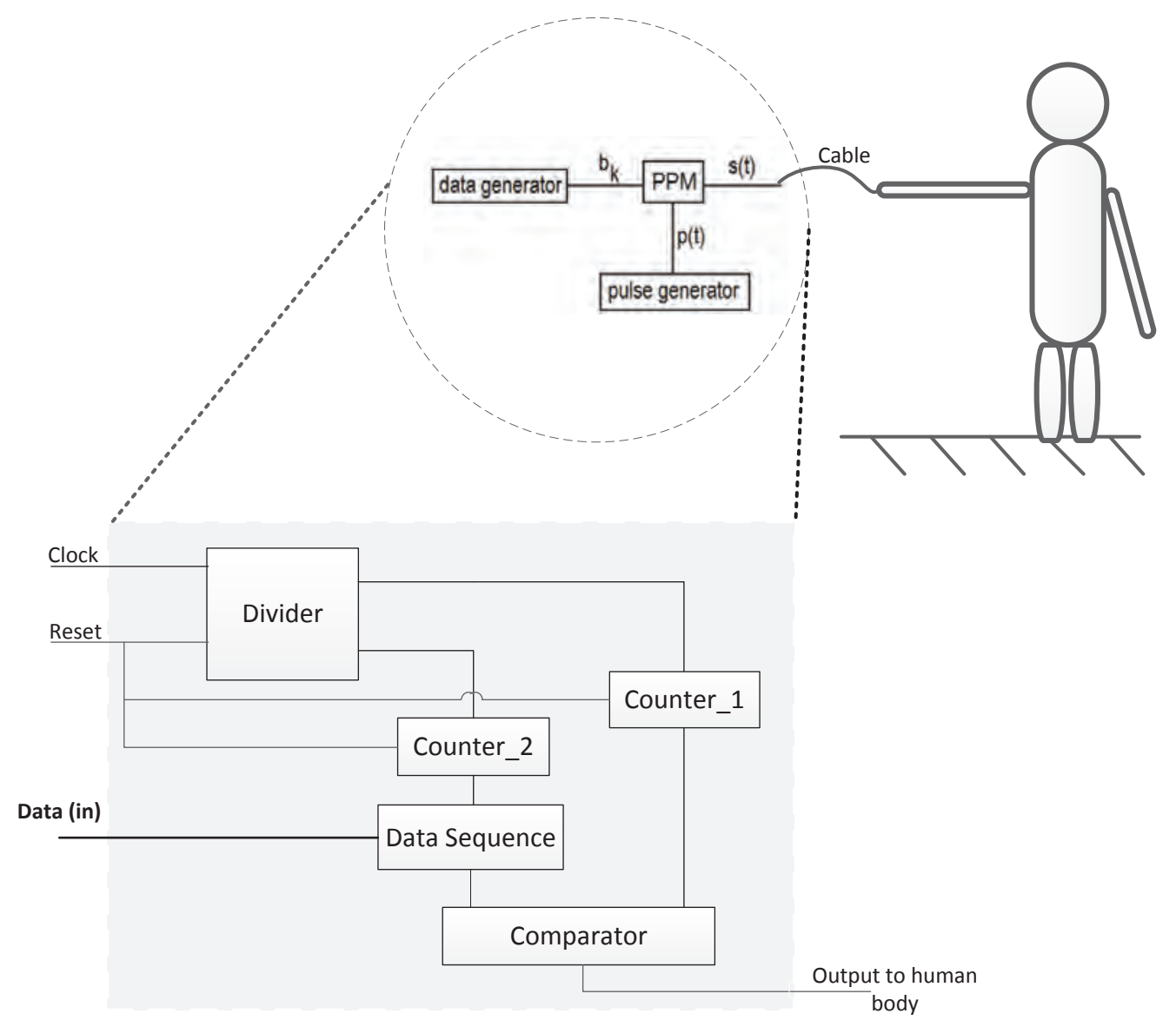

Figure 6.9: A simplified architecture of the IBC PPM transmitter.

6.9 indicates that PPM scheme could be implemented without external carrier signal requirement. Carrier-free PPM results in a simplified architecture. Through the analytical expression of the PPM signal, $s(t)$, can be achieved as follows:

$$
s(t)=\sum_{k=0}^{K} p\left(t-b_{k} \frac{T}{2}-k T\right)
$$

where $p$ and $T$ represent the basic pulse waveform and symbol period, respectively. The values of $b_{k}$ for the $k^{\text {th }}$ transmitted bit are assumed to be 0 or 1 . The transmitter 


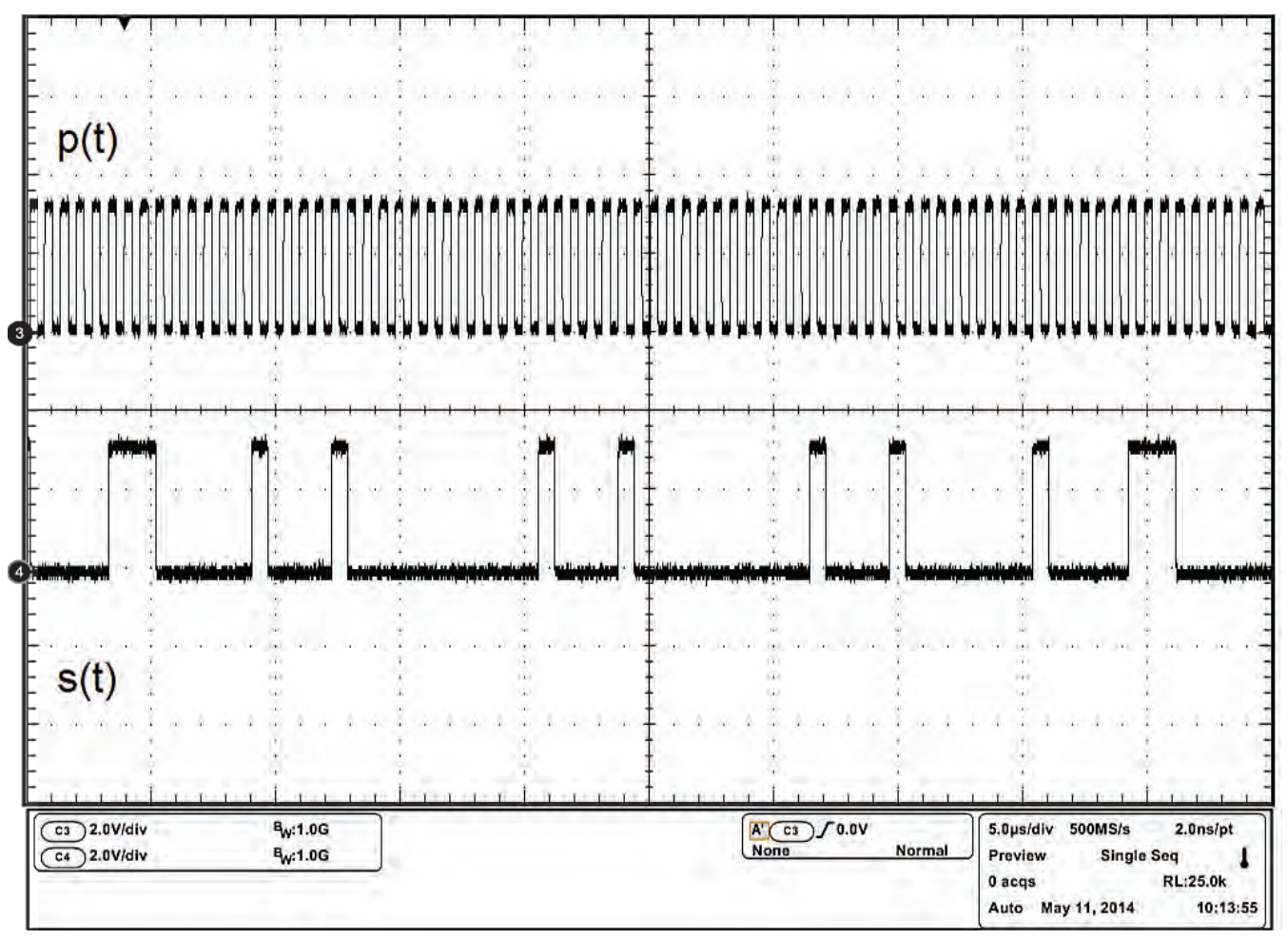

Figure 6.10: A generated PPM signal, $s(t)$, and the output of pulse generator, $p(t)$, in the IBC PPM architecture.

architecture of the IBC aims to communicate a digital signal (i.e. "Data-in" in figure 6.8) through the body after the PPM modulation. Input digital data (Data-in) consists of 3-bits which could be collected from a biomedical sensor. In fact, these data will be employed to define the positions of the active '1' in PPM coding. In this study, predefined data has been used as the input data. The $p(t)$ and $s(t)$ are plotted in figure 6.10. Also, the differential output signals from buffer (see figure 6.8) is shown in figure 6.11. The amplitude of "in+" and "in-" which are directly connected to the transmitter electrodes are $950 \mathrm{mV}$.

The IBC transmitter consists of five main components: a divider, two counters, a data sequence, and a comparator. The main clock of the FPGA (100 MHz) is 


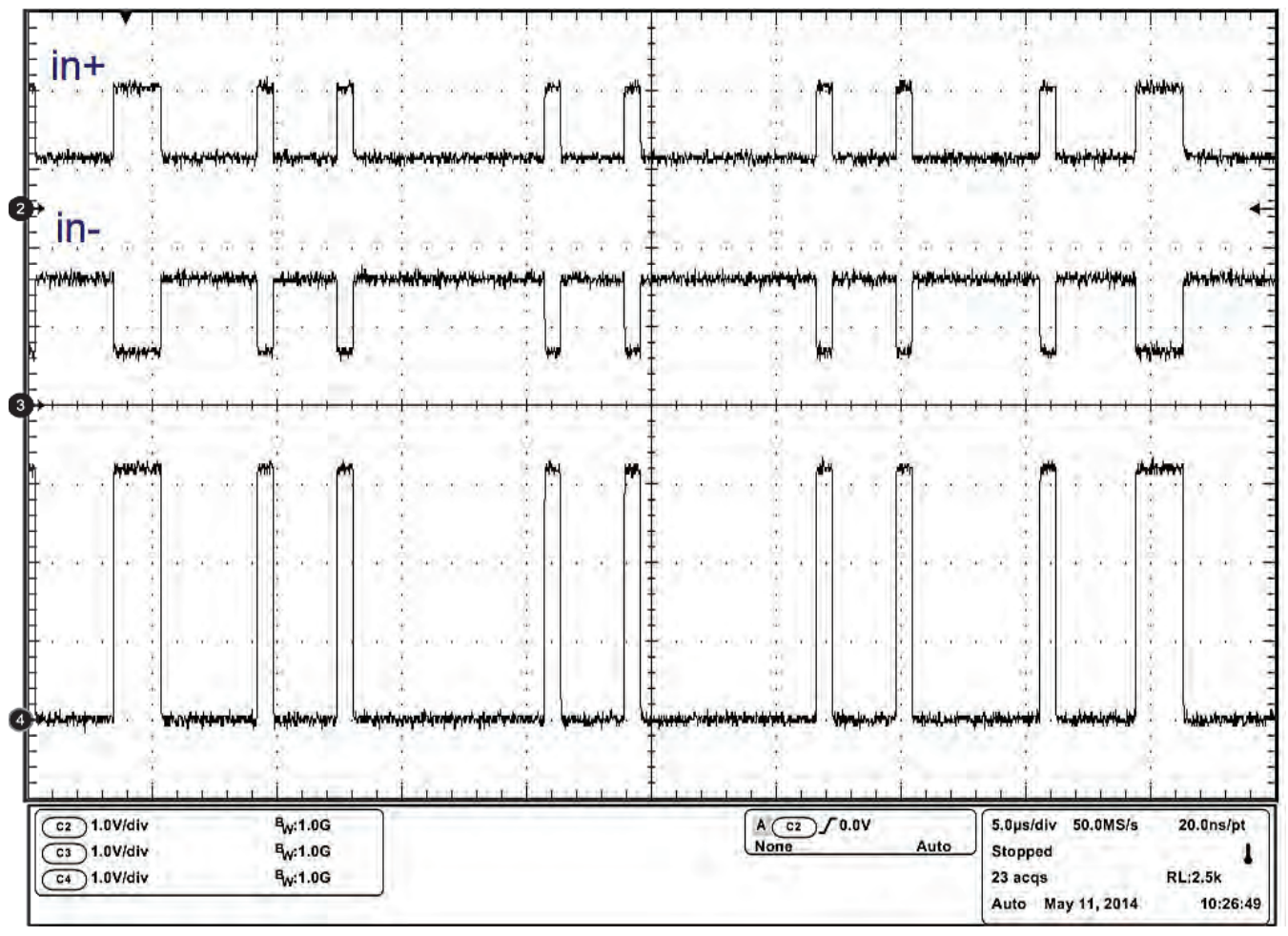

Figure 6.11: The output of IBC transmitter, in+ and in-, which transmit through the body.

divided into two different clocks by means of a divider component which activates the counter_1 and counter_2, respectively (see figure 6.9). A 24-bits "Data-in", which we assume generated by 8 biomedical sensors (3-bits per sensor) is saved in the data sequence block. Based on the output of counter_2, the number of stored information is transmitted to the comparator. Finally, the outputs of counter_1 and data sequence blocks are compared in the comparator and once the values become equal, the output of comparator will be active ' 1 '. Otherwise the output of ' 0 ' will be generated. Figure 6.11 shows the output of proposed transmitter. It is assumed that 64 slices are transmitted in each time frame. The time frame is divided into 8 time slots and each time slot contains 8 time slices encoding 8-bits. It is worth noting that for each time 


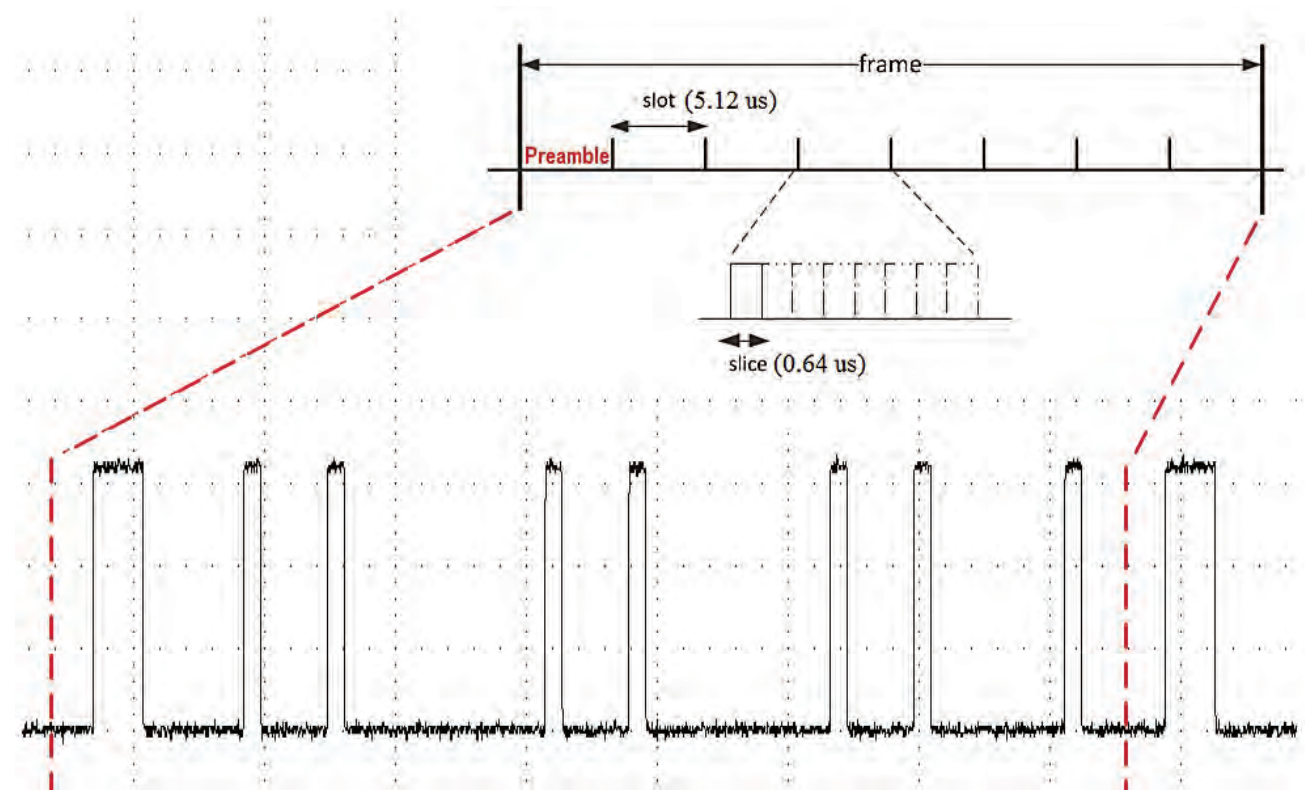

Figure 6.12: The transmitter output pattern in the proposed IBC system.

slot only 1 slice out of 8 will be activated, i.e. 8 slices per frame. Since a slice width (figure 6.12) is much shorter compared to a slice repetition cycle (frame width), the power dissipation per cycle will be low.

To generate the mentioned pattern, the FPGA configuration is specified using a very high-speed hardware description language (VHDL). The clock frequency of FPGA is divided by 64 and 512 to run the counter_1 and counter_2, respectively. We set up the counter_1 to count consecutively 8 time slices. counter_2 is also programmed to count the 8 time slots. The active slice or bit is selected by the input Data-in which stored in data sequence block. It should be noted that the first 8-bits of each frame (first time slot) is occupied by the predefined preamble sequence (i.e. "0011100" in our study) to indicate the beginnings point of each frame, before transmitting the data toward the body. 


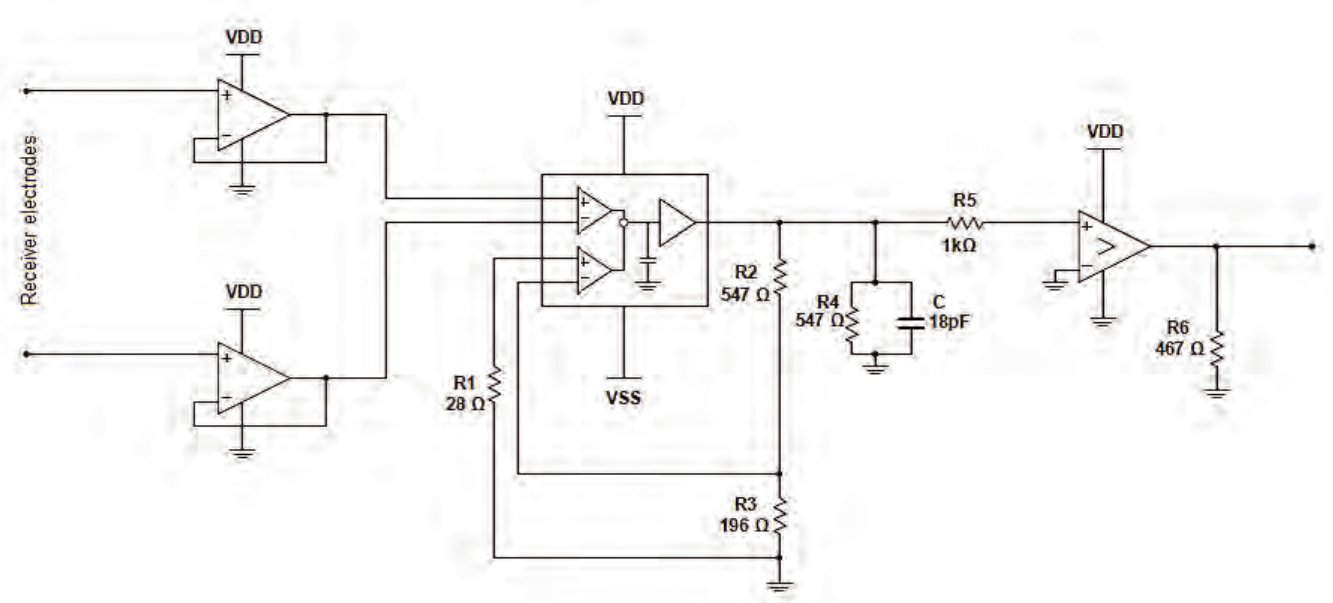

Figure 6.13: The schematic of receiver AFE used in the proposed IBC system.

\subsubsection{Analog Front-End (AFE) for Receiver}

The analog front-end (AFE) is the first stage in pursuit of the received data through the receiver electrodes. Based on our measurement results achieved in chapter 4 and the beginning of this chapter, the acquired signal from the body channel has been attenuated (between 20-80 dB). Moreover, the data has been affected by the noise of the measurement equipment and therefore needs to be removed from the received signal. Hence, the presence of filters, amplifiers, comparators, and so on is required before the data is processed by the receiver unit. Figure 6.13 shows the schematic of the proposed AFE which consists of two buffers, a differential amplifier, a comparator, based on discrete off-the-shelf components. The proposed AFE is powered by two batteries $(4.5 \mathrm{~V}$ each) to reduce the effects of power lines (50 Hz mains) on signal propagation.

Since the received data rate from the body is lower compared to the original data rate, the transmitted and received data fail to be synchronized. Therefore, first two amplifiers are used as a unity gain buffer or voltage follower. The buffers isolate 


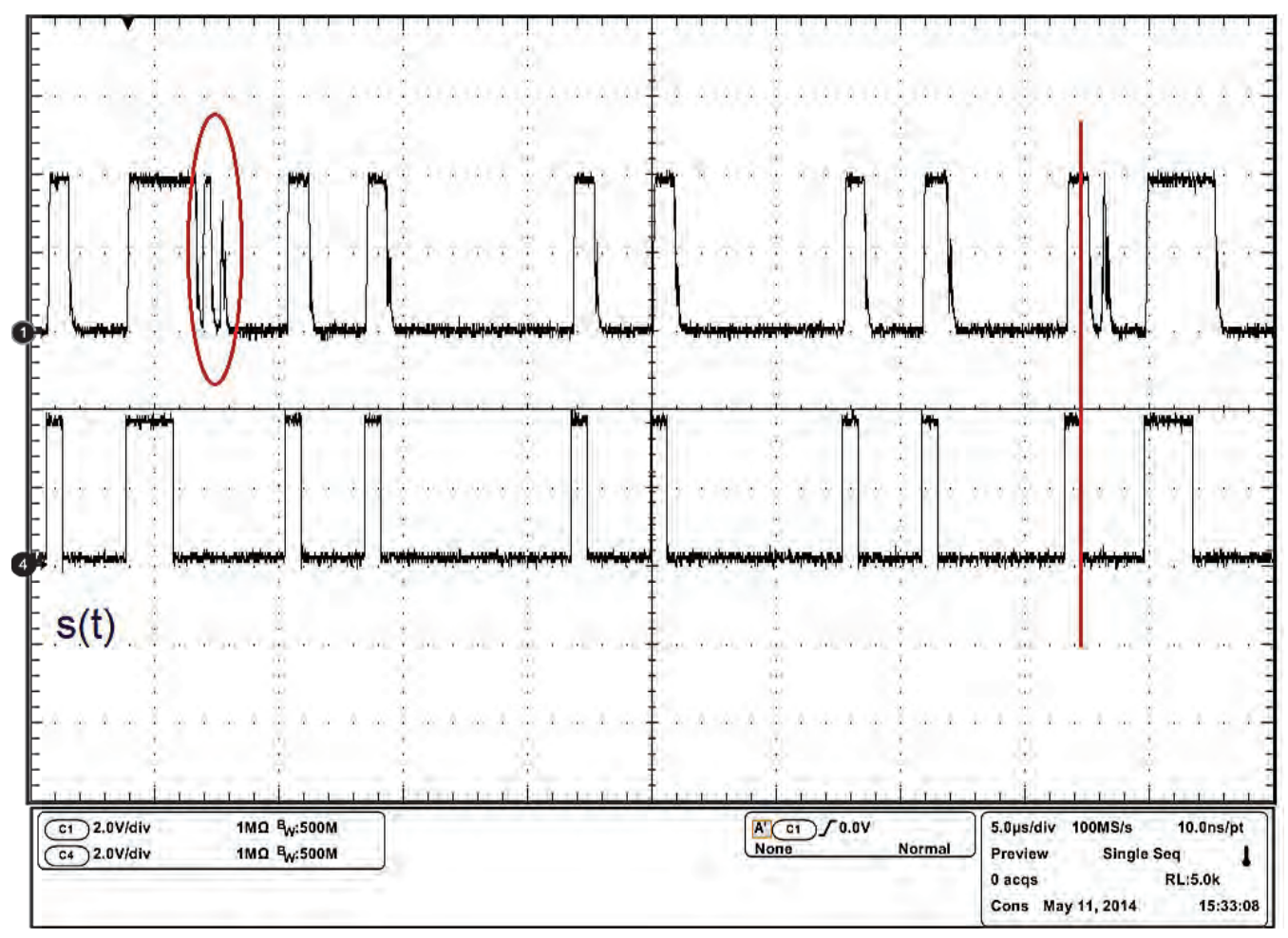

Figure 6.14: The output signal of AFE compared to the generated signal by transmitter, $s(t)$ during the body channel length of $14 \mathrm{~cm}$. The phase-offset and noise is shown in the figure.

test subjects from the receiver unit and reduce the effects of noise and distortion caused by the transmitter and the body. They also increase the ratio of common mode rejection. The differential received data from the body needs to be converted to the single-ended data to drive the receiver unit. To this end, the high speed differential amplifier (AD830, Analog Device, MA, USA) is used to deliver a wide utility gain bandwidth $(85 \mathrm{MHz})$ and a high common mode rejection ratio (65 $\mathrm{dB}$ at $5 \mathrm{MHz}$ ). The differential amplifier decouples the effects of both transmitter unit and the human body from the receiver side. According to our measurement in previous chapters, the peak to peak voltage of the received signal from body is around 80 
$\mathrm{mV}$, below $5 \mathrm{MHz}$ frequency. This poor signal is amplified by $12 \mathrm{~dB}\left(=1+\frac{R 2}{R 3}\right)$ in the differential amplifier. Further, the R1 is employed to decrease the offset of the output voltage. The differential amplifier output is connected to a comparator through a 1 $\mathrm{k} \Omega$ resister. This resistor effectively reduces the noise influence and provides a suitable signal for the comparator. The last stage of AFE is a comparator used to amplify and digitalize the signal. The low power, high speed comparator (Max941, Maxim, CA, USA) is employed in our system. The output of comparator is approximately varied between its positive and negative input terminals. In the proposed AFE, the output of comparator varies between 0 and $+3.8 \mathrm{~V}$. Figure 6.14 compares both generated signal by IBC transmitter, $s(t)$, and the AFE output signal.

\subsubsection{Receiver Unit}

The digital receiver unit of the IBC system is shown in figure 6.15. It is composed of three stages: a sampling unit, a serial to parallel (S2P) convertor, and a decoder implemented in the FPGA. The output data from AFE feeds the sampling unit of the receiver. Since this data might include a phase-offset and noise (see figure 6.14) due to time synchronization issue and the effect of body composition such as limb joint effects, the presence of sampling unit is necessary. The sampling unit calculates the

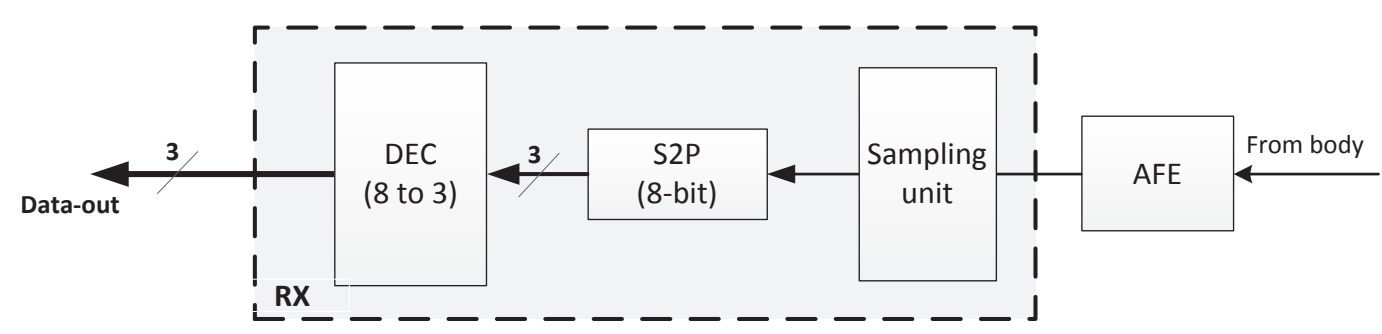

Figure 6.15: The receiver (RX) unit of the proposed IBC system. 


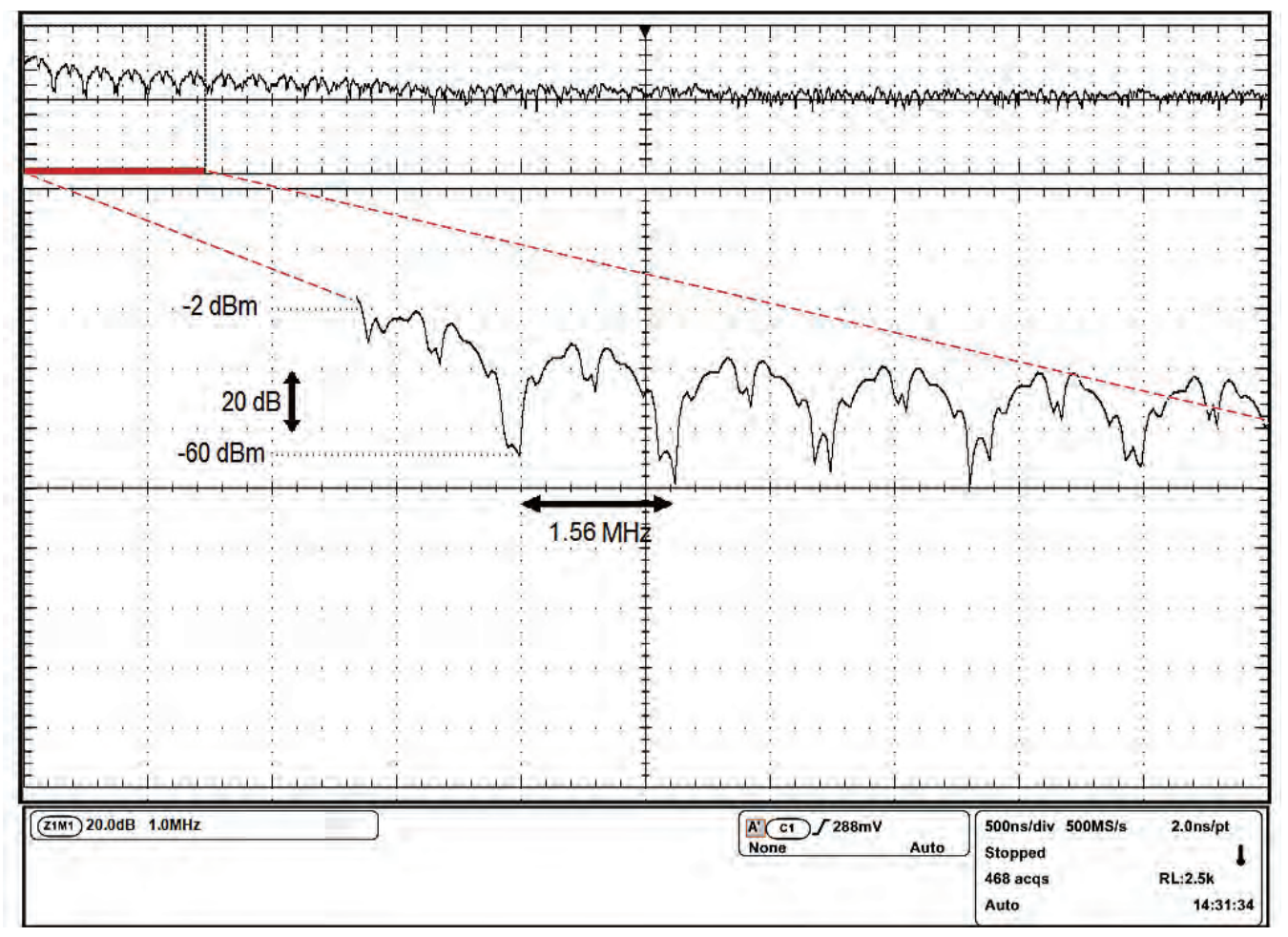

Figure 6.16: Spectrum measured at PPM transmitter output.

correct value of data after AFE, i.e. ' 1 ' or ' 0 '. Two samples from each received bit provide the correct value for the 8-bits $\mathrm{S} 2 \mathrm{P}$ shift register convertor. Once the $\mathrm{S} 2 \mathrm{P}$ is completed by 8-bits, data will flow toward decoder. Next, the 8-bits data decode to 3-bits data which is shown by the 'Data-out' in figure 6.8 and 6.14.

\subsection{Measurement Results and Discussion}

The baseband signal was modulated using PPM to transmit the digital data through the body communication channel. The maximum communication data throughput is $1.56 \mathrm{Mb} / \mathrm{s}$ when the duration of each slot is $5.12 \mu$ s which contains eight slices of 0.64 $\mu$ s duration. Figure 6.16 shows the frequency responses of the generated signal by 


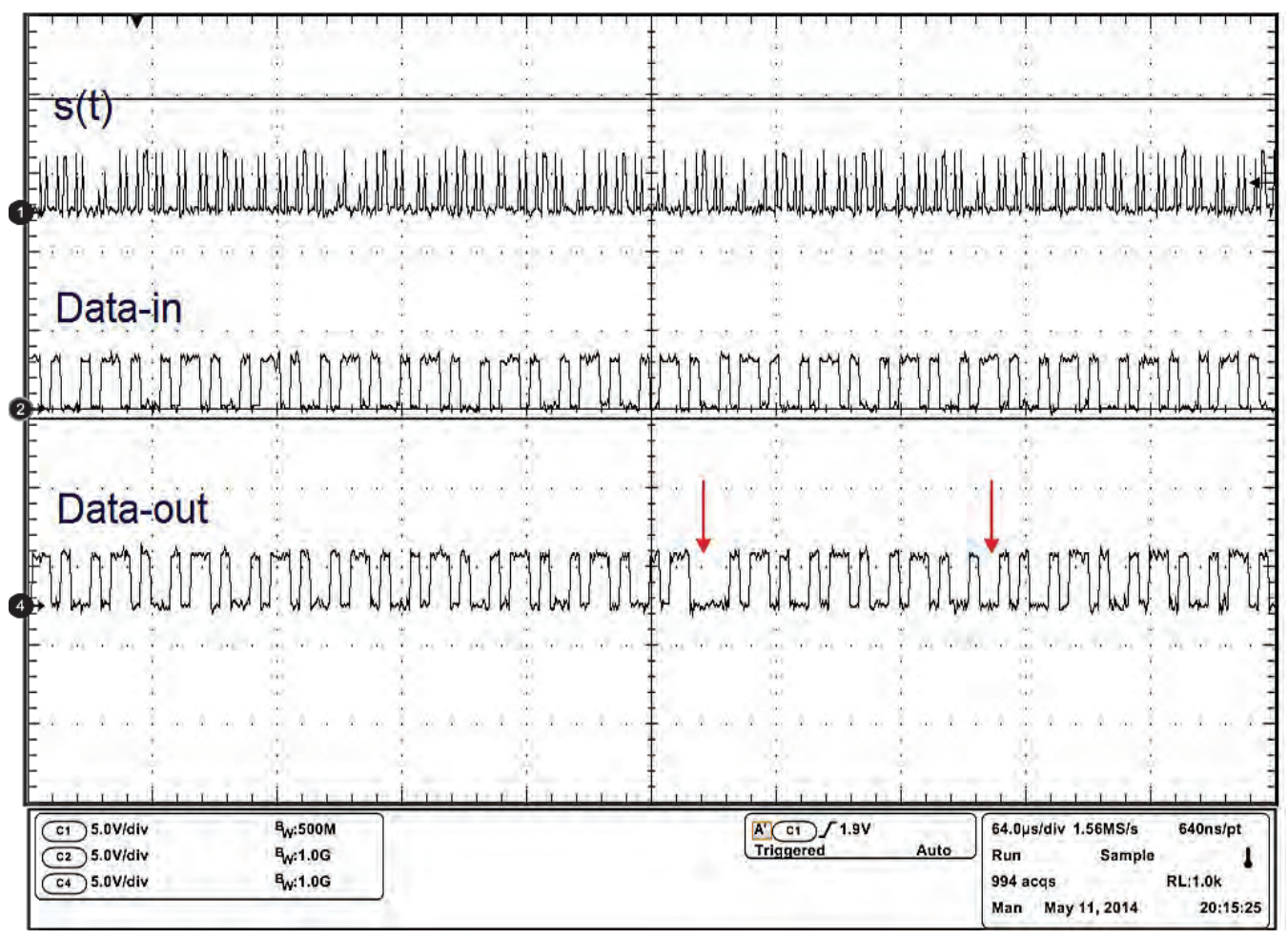

Figure 6.17: BER calculation through the measurement results of PPM IBC system. The error bits are indicated in red color.

means of the transmitter unit. The results indicate that the effect of noise becomes significant at frequency range above $18 \mathrm{MHz}$. Above this frequency, the signal is too noisy to be recognized. Therefore, the output of proposed PPM transmitter is suitable at frequencies below $18 \mathrm{MHz}$ which complies with the WBAN standard bandwidth [17].

Bit error rate (BER) quantifies the reliability of the entire communication system. To measure the BER of our IBC system, both input (Data-in) and output data captured from the receiver (Data-out) were recorded. To empirically calculate the PPM BER, the receiver electrodes were attached close to the wrist, while the transmitter electrodes were located along the arm at different positions so as to vary the distance 


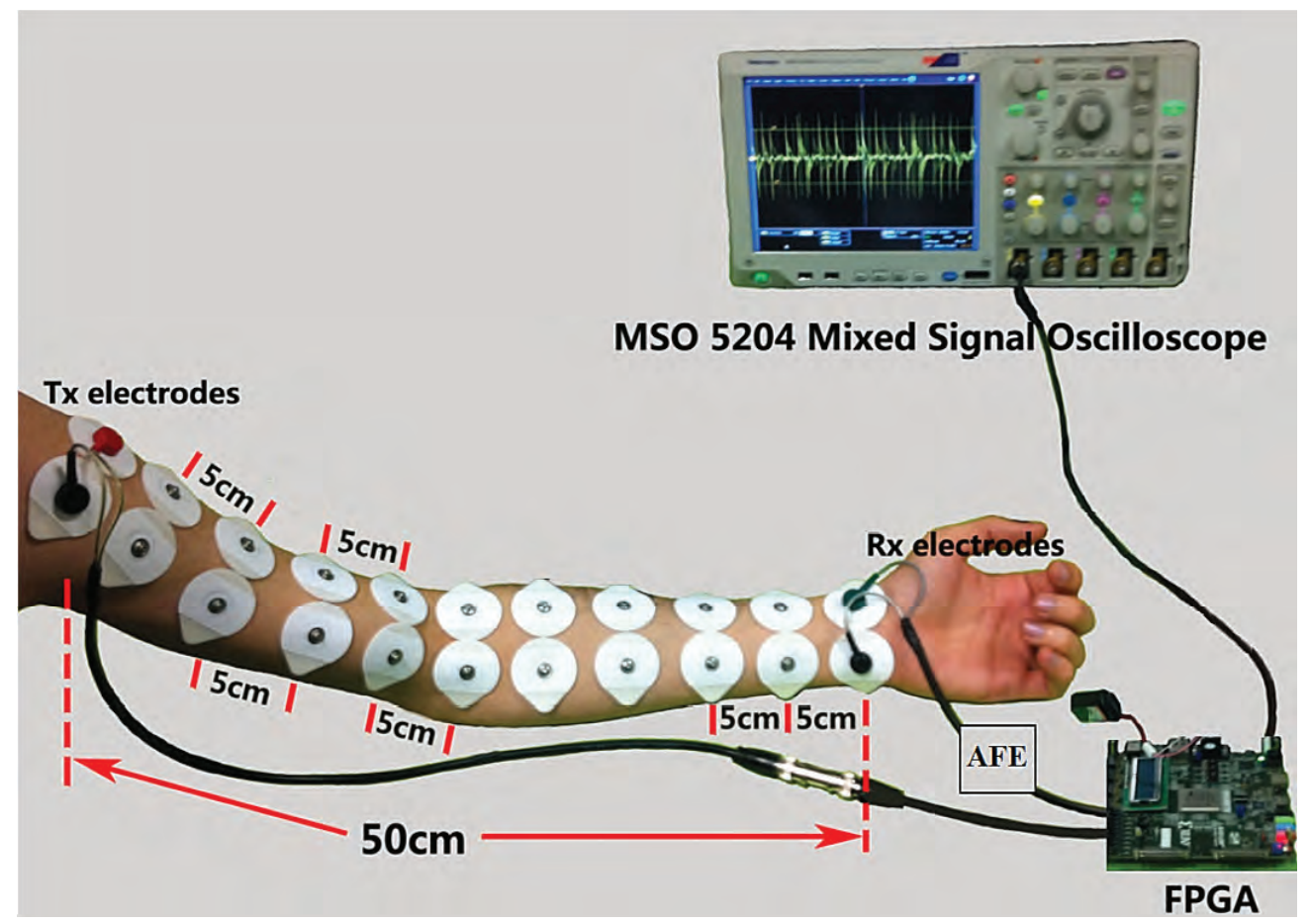

Figure 6.18: The measurement setup of galvanic coupling IBC using FPGA board for calculating the system BER.

from the receiver electrodes. The transmitter electrodes were moved along the subject's arm from 15 to $35 \mathrm{~cm}$ from receiver electrodes, i.e. the on-body channel lengths of $15 \mathrm{~cm}$ and $35 \mathrm{~cm} .250000$ stored data bit sequence from the experimental measurement were compared to calculate the BER of the IR-PPM architecture, such as figure 6.17. For instance, 187 -error bits $(7.5 \%)$ was measured during data communication through subjects' forearm. The distance between transmitter and receiver electrodes was $15 \mathrm{~cm}$. To evaluate the influence of elbow joint on the BER, the channel length was increased to $35 \mathrm{~cm}$. The results show the error-bit nearly $30 \%$ which could be due to the limb joint effect on the communicated data.

Further, the theoretical BER could be calculated using the equation (6.4) for 
L-PPM scheme:

$$
B E R=\frac{1}{2} \operatorname{erfc}\left(\frac{1}{2 \sqrt{2}} \sqrt{S N R \frac{L}{2} \log _{2} L}\right)
$$

where $L$ represents the possible pulse positions which is equal to 8 in our study. The achieved signal power in the receiver electrodes should be used to calculate the signal to noise ratio (SNR) for different distances between the transmitter and receiver electrodes. We suppose that the value of signal power is equally increasing per 5 $\mathrm{cm}$ channel lengths shown in figure 6.18. In fact, each $5 \mathrm{~cm}$ length between the transmitter and the receiver on the body arm is considered as a constant attenuator. Moreover, the power spectrum density of the additive white Gaussian noise (AWGN) of the IBC channel [106] is supposed to be constant for different on-body distances. In addition to the body noise, the measurement equipment and the measurement location noises need to be considered. To measure the value of noise, the transmitter

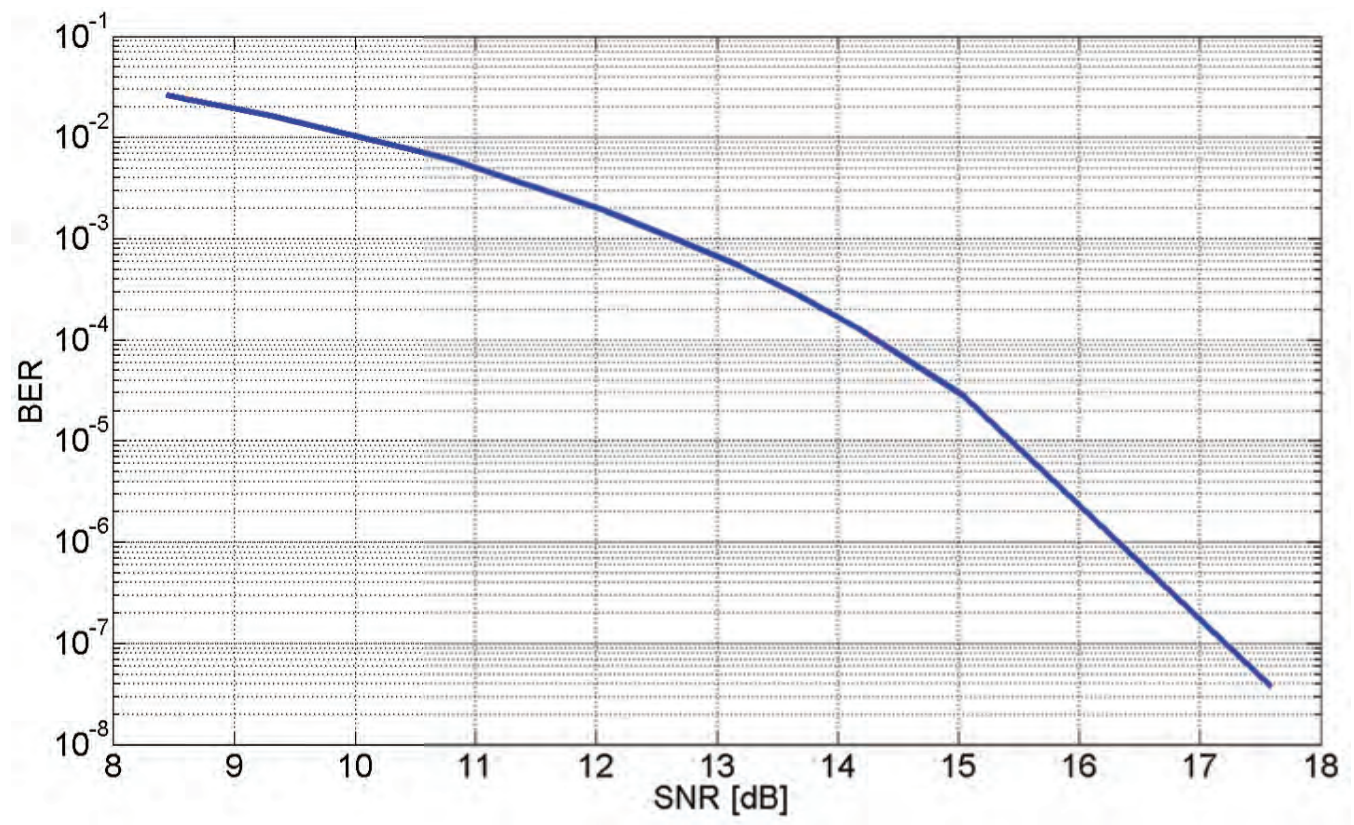

Figure 6.19: BER Performance of PPM IBC system. 
Table 6.2: The Performance Comparision of IBC Systems

\begin{tabular}{|c|c|c|c|c|}
\hline Parameters & $2007[9]$ & 2010 [108] & 2011 [67] & This work \\
\hline supply voltage & $3.3 \mathrm{~V}$ & $1.5 \mathrm{~V}$ & $0.5 \mathrm{~V}$ & $\begin{array}{c}3.3 \mathrm{~V}(\mathrm{TX}, \mathrm{RX}) \\
4.5 \mathrm{~V}(\mathrm{AFE})\end{array}$ \\
\hline $\begin{array}{l}\text { body coupling } \\
\text { method }\end{array}$ & $\begin{array}{l}\text { Galvanic } \\
\text { (FPGA) }\end{array}$ & $\begin{array}{c}\text { Capacitive } \\
\text { (ASIC-0.18 } \mu \mathrm{m} \\
\text { CMOS) }\end{array}$ & $\begin{array}{c}\text { Capacitive } \\
\text { (ASIC- } 0.18 \mu \mathrm{m} \\
\text { CMOS) }\end{array}$ & $\begin{array}{l}\text { Galvanic } \\
\text { (FPGA) }\end{array}$ \\
\hline modulation & $\begin{array}{l}\text { BPSK } \\
\text { QPSK }\end{array}$ & FSK & $\mathrm{OOK}$ & PPM \\
\hline data rate & $64 \mathrm{~kb} / \mathrm{s}$ & $1 \mathrm{Mb} / \mathrm{s}$ & $2 \mathrm{Mb} / \mathrm{s}$ & $1.56 \mathrm{Mb} / \mathrm{s}$ \\
\hline $\begin{array}{c}\text { power } \\
\text { consumption }\end{array}$ & $35 \mathrm{~mW}$ & $\begin{array}{l}2.3 \mathrm{~mW}(\mathrm{TX}) \\
3.1 \mathrm{~mW}(\mathrm{RX})\end{array}$ & $4.535 \mathrm{~mW}$ & $\begin{array}{c}2.0 \mathrm{~mW}(\mathrm{TX}, \mathrm{RX}) \\
1.94 \mathrm{~mW}(\mathrm{AFE})\end{array}$ \\
\hline energy/bit & 547 nJ/b & $3.2 \mathrm{~nJ} / \mathrm{b}$ & $2.25 \mathrm{~nJ} / \mathrm{b}$ & $1.28 \mathrm{~nJ} / \mathrm{b}$ \\
\hline
\end{tabular}

unit and the receiver electrodes were connected to the body while the transmitter unit was switched off. The oscilloscope was directly connected to on-body receiver electrodes and the observed value was $0.0028 \mathrm{~V}(-38 \mathrm{dBm})$. This value is used as the received noise power during IBC. Figure 6.19 shows the BER performance using equation (6.4). A BER of less than 0.01 is observed below $10 \mathrm{~dB}$ SNR which is acceptable value in digital communication [107].

Table 6.2, summarizes the performance of our design and compares it to the past galvanic and capacitive coupling IBC systems. While the previous galvanic coupling reported $35 \mathrm{~mW}$ of power consumption [9], our proposed transmitter and receiver consumed only $2.0 \mathrm{~mW}$ energy (estimated by Xilinx ISE Xpower analyzer) when data rate was $1.56 \mathrm{Mb} / \mathrm{s}$. Therefore, the proposed IBC system not only improves the energy efficiency of galvanic IBC methods but also could be a potential competitor for the IBC systems employing the capacitive coupling approach. The high data rate of the proposed system takes a further step forward to make it desirable for biomedical applications. 


\subsection{Summary and Conclusion}

Most of the design techniques that are developed for digital/analog communications, wireless mobile networks, digital processing techniques, and hardware designs can be readily applied to the design of IBC transceivers. However, decreasing the cost of the equipment, increasing the data rate, and fulfilling the requirements of a new WBAN standard remain significant challenges. We examined the 0.3-200 MHz frequency range for the IBC method to determine digital signal propagation characteristics at various frequency points. Based on the empirical measurements, it was found that the minimum value of body transmission channel loss was achieved at $50 \mathrm{MHz}$. In addition, the presence of a joint increased the attenuation less than $2.0 \mathrm{~dB}$ for these frequency points. In this chapter, we also focused on a baseband digital IBC system design using PPM impulse radio (PPM-IR) scheme for low frequency galvanic coupling IBC. The proposed IR-PPM architecture was implemented on FPGA board. Based on the achieved results, the presence of elbow joint within the digital data communication pathway leads to approximately $20 \%$ more error-bit compared to the channel without joint-segment. The low energy consumption per bit $1.28 \mathrm{~nJ} / \mathrm{b}$ suggests that the proposed PPM transmitter is more suitable for portable biomedical devices in the future IBC. This would help to develop a new IBC transmitter and receiver with a fully integrated ASIC (application-specific integrated circuit) architecture that has an enhanced data rate for the medical WBAN applications compared to current IBC systems. 


\section{Chapter 7}

\section{Conclusions and Future Work}

Intrabody communication (IBC) is a novel non-RF wireless data communication technique using the human body itself as the communication channel or transmission medium. In this communication method, human body acts as conductor to transmit all or a major portion of either physical or physiological data between wearable or implanted sensors around the body. The cost and energy efficiency of IBC propose it as a potential alternative technology to the high power consumption RF techniques in order to physiological data transmission around the body.

In this thesis, we focused on the influence of human body movement on IBC signal transmission considering limb joint effects. To this end, signal attenuation in IBC systems in terms of limb joint posture effects is examined in details. Also, we presented experimental and simulation results to investigate the IBC channel characteristics up to $200 \mathrm{MHz}$. Choosing higher frequencies compared to previous empirical works is to ensure that the achieved results are consistent with previous studies. Additionally, to investigate the possible effects of body movements and different environments on IBC transmissions, the wider frequency range has been employed in this work. 
Chapter 1 presented an introduction to a new ratified WBAN standard and the characteristics of its PHYs, especially IBC.

Chapter 2 compiled the latest research carried out on the novel IBC technique. Current IBC coupling methods, different IBC models, and the existed IBC hardware schemes were surveyed in this Chapter. As it stands, the IBC technique potentially offer a more power efficient and naturally secure short range communication method for body sensor networks, compared to wireless RF.

In chapter 3, the IBC safety requirements based on international guidelines were presented. The employed measurement setup and the utilized equipment in our measurements were discussed in this chapter.

In chapter 4 , the extensive empirical study results were obtained through a series of experiments on real human body. The general findings of this chapter focused on signal attenuation in IBC systems caused by limb posture effects. It studied the presence of limb joints effects on IBC, the influence of distance between the transmitter and receiver electrodes during different limb postures and motions. It also analyzed the influence of the electrode structure and their arrangements on the body during the mentioned conditions. The measurements were carried out in both indoor and outdoor environment. Understanding the dynamic body channel characteristics is crucially demanding for selecting the optimum operation frequency range and consequently determining the data transfer rate for IBC. Our experimental results show that the minimum signal attenuation was achieved between $40-60 \mathrm{MHz}$ when the body was in motion. It was concluded that while the increase in the attenuation was a function of the joint angle, the presence of joint increases the attenuation by an approximately constant amount. It was also found that. Moreover, the effect of different locations on IBC measurements can be neglected. Finally, it was concluded that the 
performance of IBC during the two-electrode arrangement will be more susceptible to motion while the four-electrode arrangement is affected by the body composition of the IBC user.

In chapter 5 , we proposed a new IBC channel modeling in order to increase the efficiency of the technical aspects of IBC. The proposed models address two major deficiencies of previous IBC circuit models. First, they take the limb joint effects of the body into account which underlies the future investigation of more advanced posture adapted IBC system design. Second, the proposed models consider the influence of measurement equipment in higher frequency band through covering wider frequency ranges. Based on the achieved measurement and simulation results, it was obtained that the joint presence between the forearm and the upper arm leads to an increment in signal attenuation at low frequencies $(<54 \mathrm{MHz})$. It was also found that the measurement equipment behave as an antenna at higher frequencies above $54 \mathrm{MHz}$. Simulation results indicated that the constant increasing of a joint length leads to attenuation growth by an approximately constant value of $0.6 \mathrm{~dB} / \mathrm{cm}$, below $20 \mathrm{MHz}$.

Chapter 6 focused on the study of IBC system component, IBC system design, and implementation. We first examined the transmission characteristics of a pulse wave at high frequency range of 0.3-200 MHz during IBC method. Furthermore, we proposed the carrier-free scheme based on the high frequency impulse radio (IR) for the IBC system design. The PPM modulation scheme was employed to implement the IRIBC architecture on the battery-powered FPGA board. The low-energy consumption per bit $1.28 \mathrm{~nJ} / \mathrm{b}$ and the low BER performance (less then $10 \%$ for the $15 \mathrm{~cm}$ onbody channel) suggests that the proposed IR-IBC could be more suitable for WBAN biomedical devices in the future. 


\subsection{Challenges of the IBC System Design}

IBC is relatively a new communication technology and has been investigated for less than two decades by several researcher groups. Although it is ratified in a standard, there are still some major remaining challenges to be addressed. The transmission characteristics of the human body is the key feature for designing a high data rate, energy and cost efficient IBC system. In order to achieve this, understanding the signal path loss and signal propagation through the body is paramount. Despite the existence of different human body communication channel modeling approaches, the empirical measurement has generally failed to be conformed to the proposed models. Majority of the proposed models did not fully considered the dielectric properties of tissues, which determine the accurate human body response to applied electrical stimulus. Moreover, tissue dispersions and dielectric spectrums have not been addressed to suit the frequency range of the proposed models. Another important factor that has not been adequately emphasized despite its pivotal role in signal coupling and signal attenuation is the coupling impedance between the electrodes and the human skin. On top of that, there is no sufficient analysis and models that could explain the created impedance by the electrode-skin contact within the context of IBC. Some of the proposed measurement procedures have not reflected all the conditions in the proposed theoretical models; e.g., little attention has been given to the implementation of a grounding strategy that matches the correct galvanic or capacitive coupling outline. Also, the proposed models have failed to take the subject specific anatomical parameters, such as subject anthropometry into account. Finally, the models are not succeeded to assess the effects of all devices involved in measurement set-ups.

A primary aim of the IBC architecture design, similar to all communications systems, is to provide a highest possible data rate. For this purpose, a high carrier 
frequency and a wide frequency bandwidth are necessary. Although different frequency ranges and bandwidths were considered in IBC transceivers, no specific study has investigated the optimal frequency range for IBC systems. In general, two significant factors that determine the frequency range of capacitive coupling IBC are the carrier wavelength $(\lambda)$, and the electromagnetic interference. For frequencies above $300 \mathrm{MHz}$, the wavelength of the carrier signal gets comparable to size of the human body. Consequently, the body behaves as an antenna; therefore, significant channel variation will appear. On the other hand, frequencies below $100 \mathrm{kHz}$ are vulnerable to all kinds of electromagnetic interference. The optimum range for the capacitive coupling method is therefore between these two frequencies. In the galvanic method, the frequency range is mostly determined by the dielectric properties of human tissues. According to Gabriel et al. [1], 0.1-10 MHz is suggested as a suitable frequency range in which the dielectric properties of most human body tissues are almost constant. The reduced bandwidth compared to the capacitive method implies a lower data throughput. Therefore, improving data throughput within these frequency ranges is of highest priority for current and future IBC transceiver designs.

Finally, since various postures are encountered during the complex human body movement such as walking, the IBC system should be adaptable to divergent body motions. Although motion effects in radio frequency (RF) communication systems have been reported, body motion in IBC transceiver design has been insignificantly considered. It has been known that body movement affects data transmission, however, the variations of channel capacity during the body motion has not as yet been studied or modeled. Human body channel models are also required for determining various transmission channel limitations and the conditions under which these limitations occur. The investigation of channel limitations will inevitably raise further 
concerns about transmitter and receiver design.

\subsection{Future Work and Future Directions}

IBC as a newborn technique is in its initial developmental stages. The field is still suffering from major and growing concerns which needs to be addressed in future investigations. The forthcoming attempts in this field may explore theoretical models to understand the body channel characteristics and constraints. In addition, complex and dynamic body limb structures as well as the environmental effects of body's vicinity on a secure data communication leave significant room for future discussions on the current empirical results to further enhance the forthcoming models. Further measurements on the various groups of test subjects with different age range would involve proper feature selection method for highly efficient channel modeling. Additional human channel modeling will also be necessary while the body is in motion. This would help to develop an adaptable body movement IBC transceiver that are mainly based on low power consumption, enhanced data rate, and small form factor for ease of use in the biomedical applications compared to current IBC systems.

A promising approach for IBC (capacitive coupling or galvanic coupling) together with an effective and accurate transceiver circuit design based on the technical details such as noise and interference must be considered in the future IBC system design. In addition, since IBC is one of the physical layers (PHYs) of the new wireless body area network (WBAN) IEEE 802.15.6 standard, the specification of WBAN such as quality-of-service (QoS) scalability and network coexistence needs to be considered in future IBC system design. The future IBC systems will be optimized with the aim of 
utilizing it networking for monitoring a variety of health parameters. Successful developments will improve portable health monitoring through the use of multiple wearable or implanted sensors on the human body (sensor networks) and the development of low power data transmission methods. Future work will include a development of a body posture adapted IBC transceiver based on the achieved results in this work. The technology will increase patient convenience and improve the cost effectiveness of health care through wireless portable monitoring. Table 7.1 shows the summary of challenges and future work which should be considered in the IBC area.

Table 7.1: Summary of Challenges and Future Work in the IBC Technique

\begin{tabular}{|c|c|}
\hline Challenges & Future Work \\
\hline $\begin{array}{l}\text { The frequency dispersions of human } \\
\text { tissue limits maximum carrier frequen- } \\
\text { cies and data rate. }\end{array}$ & $\begin{array}{l}\text { New techniques to improve data rates } \\
\text { in low frequency ranges required. }\end{array}$ \\
\hline $\begin{array}{l}\text { The coupling impedance between the } \\
\text { electrodes and the human skin }\left(Z_{E S}\right) \\
\text { is difficult to determine, but greatly af- } \\
\text { fects the transmission quality. }\end{array}$ & $\begin{array}{l}\text { New electrode-body impedance match- } \\
\text { ing schemes required to maximize the } \\
\text { power transfer. }\end{array}$ \\
\hline $\begin{array}{l}\text { Channel models incorporating the hu- } \\
\text { man body movement required. In ad- } \\
\text { dition, the effects of all component de- } \\
\text { vices in the measurement setup should } \\
\text { be considered. }\end{array}$ & $\begin{array}{l}\text { Theoretical models to understand the } \\
\text { body channel characteristics and con- } \\
\text { straints. Human channel modeling will } \\
\text { be necessary while the body is in mo- } \\
\text { tion. }\end{array}$ \\
\hline Effect of long term use & $\begin{array}{l}\text { Studies on tissue effects for IBC use in } \\
\text { the long term, e.g. Cancer. }\end{array}$ \\
\hline $\begin{array}{l}\text { Transceiver specifications based on } \\
\text { IEEE 802.15.6 standard (low power } \\
\text { consumption, transmit mask, receiver } \\
\text { sensitivity, and signal modulation and } \\
\text { coding) difficult to fulfill. }\end{array}$ & $\begin{array}{l}\text { The specification of IEEE } 802.15 .6 \\
\text { needs to be considered in future IBC } \\
\text { transceiver design. }\end{array}$ \\
\hline $\begin{array}{l}\text { Networking issues such as security for } \\
\text { information, QoS, inter-relationship in } \\
\text { different environments, and mobility. }\end{array}$ & $\begin{array}{l}\text { New transceiver design based on high } \\
\text { security, low power consumption, en- } \\
\text { hanced data rate, and small form factor } \\
\text { for ease of use compared to current IBC } \\
\text { systems. }\end{array}$ \\
\hline
\end{tabular}




\section{References}

[1] S. Gabriel, R. Lau, and C. Gabriel, "The dielectric properties of biological tissues: Iii. parametric models for the dielectric spectrum of tissues," Phys. Med. Biol., vol. 41, no. 11, pp. 2271-2293, Nov. 1996.

[2] J. M. Smith, "The doctor will see you always," IEEE Spectrum Magazine, vol. 48, no. 10, pp. 51-55, Oct. 2011.

[3] J. C. Kvedar, Heart Failure Management using Telemonitoring, [Online] Available: www.ehcca.com/presentations/readsummit1/kvedar_2.pdf, 2009.

[4] Connecting Health Services with the Future: Modernising Medicare by Providing Rebates for Online Consultations, [Online] Available: $\quad$ www.health.gov.au/internet/mbsonline/publishing.nsf/Content/ 256BA3C38B7EEA22CA2577EA006F7C42/\$File/CHSWTFsub-MTAA.pdf, 2011.

[5] A. Dhamdhere, H. Chen, A. Kurusingal, V. Sivaraman, and A. Burdett, "Experiments with wireless sensor networks for real-time athlete monitoring," in IEEE Local Computer Networks (LCN), Oct. 2010, pp. 938-945. 
[6] 10 Wearable Health Tech Devices To Watch, [Online] Available: http://www. techweb.com/news/240012613/10-wearable-health-tech-devices-to-watch. html, 2012.

[7] H. W. Winston, A. T. B. Alex, A. B. Maxim, D. L., and J. K. William, "Incremental diagnosis method for intelligent wearable sensor systems," IEEE Trans. Inf. Technol. Biomed., vol. 11, no. 5, pp. 553-562, Sep. 2007.

[8] B. Mariani, M. C. Jimenez, F. Vingerhoets, and K. Aminian, "On-shoe wearable sensors for gait and turning assessment of patients with parkinsons disease," IEEE Trans. Biomed. Eng., vol. 60, no. 1, pp. 155-158, Jan. 2013.

[9] M. S. Wegmueller, "Intra-Body Communication for Biomedical Sensor Networks," Ph.D. dissertation, ETH Zurich, Switzerland, 2007.

[10] M. R. Yuce and T. Dissanayake, "Easy-to-swallow wireless telemetry," IEEE Microw. Magazine, vol. 13, no. 6, pp. 90-101, Oct. 2012.

[11] U. Varshney, "Pervasive healthcare and wireless health monitoring," Mobile Netw. Appl., no. 12, pp. 113-127, Jul. 2007.

[12] M. Lauzier, P. Ferrand, H. Parvery, A. Fraboulet, and J.-M. Gorce, "WBANs for live sport monitoring: An experimental approach, early results and perspectives," in EURO-COST IC1004, Sep. 2012, pp. 1-6.

[13] S. Adibi, "Link technologies and blackberry mobile health (mhealth) solutions: A review," IEEE Trans. Inf. Technol. Biomed., vol. 16, no. 4, pp. 586-597, Jul. 2012 . 
[14] M. Patel and J. Wang, "Applications, challenges, and prospective in emerging body area networking technologies," IEEE Commun. Magazine, vol. 17, no. 1, pp. 80-88, Feb. 2010.

[15] M. Hanson, H. Powell, A. Barth, K. Ringgenberg, B. Calhoun, J. Aylor, and J. Lach, "Body area sensor networks: Challenges and opportunities," Computer, vol. 42, pp. 58-65, Jan. 2009.

[16] J. Wang and Q. Wang, Body Area Communications: Channel Modeling, Communication Systems, and EMC. Singapore : Wiley (Asia), 2013.

[17] "IEEE Standard for Local and Metropolitan Area Networks Part 15.6: Wireless Body Area Networks," IEEE Std. 802.15.6-2012, Feb. 2012.

[18] H.-J. Yoo, "Wireless body area network and its healthcare applications," in IEEE Asia-Pacific Microwave Conf. (APMC'13), Nov. 2013, pp. 89-91.

[19] L. Liu, R. D'Errico, L. Ouvry, P. De Doncker, and C. Oestges, "Dynamic channel modeling at $2.4 \mathrm{GHz}$ for on-body area networks," ADVANCES IN ELECTRON. AND TELECOMMUN., vol. 2, no. 4, pp. 18-27, Dec. 2011.

[20] M. S. Wegmueller, M. Oberle, N. Felber, and W. Kuster, N. Fichtner, "Signal transmission by galvanic coupling through the human body," IEEE Trans. Instrum. Meas., vol. 59, no. 4, pp. 963-969, Apr. 2010.

[21] J. Bae, K. Song, H. Lee, H. Cho, and H.-J. Yoo, "A low-energy crystal-less double-FSK sensor node transceiver for wireless body-area network," IEEE J. Solid-State Circuits, vol. 47, no. 11, pp. 2678-2692, Nov. 2012. 
[22] R. Xu, N. W. Chiu, H. Zhu, S. Hengying, and J. Yuan, "Equation environment coupling and interference on the electric-field intrabody communication channel," IEEE Trans. Biomed. Eng., vol. 59, no. 7, pp. 2051-2059, Jul. 2012.

[23] T. Falck, H. Baldus, J. Espina, and K. Klabunde, "Plug'n play simplicity for wireless medical body sensors," Mobile Netw. and Appl., vol. 12, pp. 143-153, Jul. 2007.

[24] N. Cho, L. Yan, J. Bae, and H.-J. Yoo, "A 60-kb/s-10-Mb/s adaptive frequency hopping transceiver for interference-resilient body channel communications," IEEE J. Solid-State Circuits, vol. 44, no. 3, pp. 708-717, Mar. 2009.

[25] T. Kang, I. Lim, J. Hwang, C. Hyoung, H. Park, and S. Kang, "A method of increasing data rate for human body communication system for body area network applications," in IEEE Vehicular Technology Conf. (VTC Fall), Sep. 2012, pp. 1-5.

[26] M. Shinagawa, J. Katsuyama, K. Matsumoto, S. m. a. Hasegawa, T. Yanase, R. Sugiyama, and Y. Kado, "Noise analysis for near-field intra-body communication systems," in IEEE Int. Instrum. Meas. Technol. Conf. (I2MTC), May. 2013, pp. 902-907.

[27] M. A. Callejon, D. Naranjo-Hernandez, J. Reina-Tosina, and L. M. Roa, "A comprehensive study into intrabody communication measurements," IEEE Trans. Instrum. Meas., vol. 62, no. 9, pp. 2446-2455, Sep. 2013.

[28] N. Zedong, M. Jingjing, C. Hong, and W. Lei, "Statistical characterization of the dynamic human body communication channel at $45 \mathrm{MHz}$," in IEEE 28th Annu. Int. Conf. EMBC, Jul. 2013, pp. 1206-1209. 
[29] S. Schaal, "Dynamic movement primitives - A framework for motor control in humans and humanoid robotics," in Adaptive Motion of Animals and Machines, H. Kimura, K. Tsuchiya, A. Ishiguro, and H. Witte, Eds. Springer, 2006.

[30] E. N. Marieb, Human Anatomy and Physiology. San Francisco: Benjamin Cummings Press, 2001.

[31] T. C. W. Schenk, N. S. Mazloum, L. Tan, and P. Rutten, "Experimental characterization of the body-coupled communications channel," in IEEE Int. Symp. Wireless Communication Systems (ISWCS '08), Oct. 2008, pp. 234-239.

[32] M. A. Callejon, D. Naranjo-Hernandez, J. Reina-Tosina, and L. M. Roa, "Distributed circuit modeling of galvanic and capacitive coupling for intrabody communication," IEEE Trans. Biomed. Eng., vol. 59, no. 11, pp. 3263-3269, Nov. 2012.

[33] K. Y. Yazdandoost, K. Sayrafian-Pour, and K. Hamaguchi, "RF propagation and channel modeling for UWB wearable devices," IEICE Trans. Commun., vol. E94-B, no. 5, pp. 1126-1134, May. 2011.

[34] H. B. Lim, D. Baumann, and E.-P. Li, "A human body model for efficient numerical characterization of UWB signal propagation in wireless body area networks," IEEE Trans. Biomed. Eng., vol. 58, no. 3, pp. 689-697, Mar. 2011.

[35] S.-J. Song, "Energy-Efficient Body-Coupled Data Transceiver for Body Area Networks," Ph.D. dissertation, KAIST Daejeon, South Korea, 2007.

[36] K. Van Dam, M. Pitchers, and M. Barnard, "Body area networks: towards a wearable future," in Wireless World Research Forum (WWRF) Kick Kff Meeting, Mar. 2001. 
[37] H.-J. Yoo, "Your heart on your sleeve," IEEE Solid-State Circuits Magazine, vol. 5, no. 1, pp. 59-70, Feb. 2013.

[38] B. Dolan, U.S. Home-Based Wireless Healthcare Market: \$4.4B in 2013, [Online] Available: http://mobihealthnews.com/3727/ us-home-based-wireless-healthcare-market-44b-in-2013/, 2009.

[39] T. G. Zimmerman, "Personal area networks: near-field intrabody communication," IBM Syst., vol. 35, no. 3-4, pp. 609-617, Apr. 1996.

[40] M. Oberle, "Low Power Systems-on-Chip for Biomedical Applications," Ph.D. dissertation, ETH Zurich, Switzerland, 2002.

[41] N. Cho, J. Yoo, S. Song, J. Lee, S. Jeon, and H.-J. Yoo, "The human body characteristics as a signal transmission medium for intra-body communication," IEEE Trans. Microw. Theory Tech., vol. 55, no. 5, pp. 1080-1086, May 2007.

[42] H. Baldus, S. Corroy, A. Fazzi, K. Klabunde, and T. Schenk, "Human-centric connectivity enabled by body-coupled communications," IEEE Commun. Magazine, vol. 47, no. 6, pp. 172-178, Jun. 2009.

[43] S. Pun, Y. Gao, P. Mak, M. Vai, and M. Du, "Quasi-static modeling of human limb for intra-body communications with experiments," IEEE Trans. Inf. Technol. Biomed, vol. 15, no. 6, pp. 870-876, Nov. 2011.

[44] J. D. Bronzino, The Biomedical Engineering Handbook: Biomedical Engineering Fundamentals. Boca Raton: CRC Press, 2011.

[45] H. P. Schwan, "Electrical properties of tissue and cell suspensions," Adv. Biol. Med. Phys., vol. 5, pp. 147-209, 1957. 
[46] R. Xu, H. Zhu, and J. Yuan, "Electric-field intra-body communication channel modeling with finite-element method," IEEE Trans.Biomed. Eng., vol. 58, no. 3, pp. 705-712, Mar. 2011.

[47] K. S. Cole and R. H. Cole, "Dispersion and absorption in dielectricspart i: Alternating current characteristics," J. Chem. Phys., vol. 9, no. 4, pp. 341-351, Apr. 1941.

[48] D. Miklavcic, N. Pavselj, and F. Hart, "Electric properties of tissues," in Wiley Encyclopedia of Biomedical Engineering. New York: Wiley Press, 2006.

[49] A. Ivorra, "Bioimpedance monitoring for physicians: An overview," Centre Nacional de Microelectronica: Biomed. Appl. Group, Jul. 2003.

[50] S. Grimnes and O. G. Martinsen, Bioimpedance and Bioelectricity Basics. U.K: Academic Press, 2008.

[51] Skin Effect and Bio-Electrical Impedance Analysis, [Online] Available: http:// www.rifevideos.com/pdf/skin_effect_and_bio_electrical_impedance_analysis.pdf, 2011.

[52] H. Kanai, M. Haeno, and K. Sakamoto, "Electrical measurement of fluid distribution in legs and arms," Med. Prog. Technol., vol. 12, no. 3-4, pp. 159-170, 1987.

[53] M. Callejon, L. Roa, J. Reina-Tosina, and D. aranjo Hernandez, "Study of attenuation and dispersion through the skin in intra-body communications systems," IEEE Trans. Inf. Technol. Biomed., vol. 16, no. 1, pp. 159-165, Jan. 2012. 
[54] K. Hachisuka, Y. Terauchi, Y. Kishi, T. Hirota, K. Sasaki, H. Hosaka, and K. Ito, "Simplified circuit modeling and fabrication of intrabody communication devices," Sens. Actuators A, vol. 130-131, pp. 322-330, Jun. 2006.

[55] J. Bae, K. Song, H. Lee, H. Cho, and H.-J. Yoo, "A 0.24-nJ/b wireless bodyarea-network transceiver with scalable double-fsk modulation," IEEE J. SolidState Circuits, vol. 47, no. 1, pp. 310-322, Jan. 2012.

[56] W. Besio and A. Prasad, "Analysis of skin-electrode impedance using concentric ring electrode," in IEEE 28th Annu. Int. Conf. EMBC, Aug. 2006, pp. 64146417.

[57] Y. Song, Q. Hao, K. Zhang, M. Wang, Y. Chu, and B. Kang, "The simulation method of the galvanic coupling intra-body communication with different signal transmission paths," IEEE Trans. Instrum. Meas., vol. 60, no. 4, pp. 1257-1266, Apr. 2011.

[58] R. Xu, H. Zhu, and J. Yuan, "Circuit-coupled FEM analysis of the electricfield type intra-body communication channel," in IEEE Biomed. Circuits Syst. Conf., Nov. 2009, pp. 221-224.

[59] M. Callejon, J. Reina-Tosina, D. aranjo Hernandez, and L. Roa, "Galvanic coupling transmission in intra-body communications: A finite element approach," IEEE Trans. Biomed. Eng., vol. 61, no. 3, pp. 775-783, Mar. 2014.

[60] K. Fujii, M. Takahashi, and K. Ito, "Electric field distributions of wearable devices using the human body as a transmission channel," IEEE Trans. Antennas Propag., vol. 55, no. 7, pp. 2080-2087, Jul. 2007. 
[61] K. Hachisuka, A. Nakata, T. Takeda, Y. Terauchi, K. Shiba, K. Sasaki, H. Hosaka, and K. Itao, "Development and performance analysis of an intrabody communication device," in IEEE 12th Int. Conf. Solid-State Sens., Actuators Microsyst., Jun. 2003, pp. 1722-1725.

[62] J. Bae, K. Song, H. Lee, H. Cho, and H.-J. Yoo, "The signal transmission mechanism on the surface of human body for body channel communication," IEEE Trans. Microw. Theory Tech., vol. 60, no. 3, pp. 582-593, Mar. 2012.

[63] X. Chen, P. Mak, S. Pun, Y. Gao, M. Vai, and M. Du, "Signal transmission through human muscle for implantable medical devices using galvanic intrabody communication technique," in IEEE 34th Annu. Int. Conf. EMBC, Aug. 2012, pp. 1651-1654.

[64] B. P. Lathi and Z. Ding, Modern Digital and Analog Communication Systems. New York: Oxford Univ. Press, 2010.

[65] S. Mishra, Brief overview of network transceivers, [Online] Available: http: //ezinearticles.com/?Brief-Overview-of-Network-Transceivers\&id=5315342, 2011.

[66] A. Fazzi, S. Ouzounov, and J. van den Homberq, "A $2.75 \mathrm{~mW}$ wideband correlation-based transceiver for body-coupled communication," in IEEE Int. Solid-State Circuits Conf. Dig. Tech. Papers, Feb. 2009, pp. 204-205.

[67] Y.-T. Lin, Y.-S. Lin, C.-H. Chen, H.-S. Chen, Y.-C. Yang, and S.-S. Lu, "A 0.5-V biomedical system-on-a-chip for intrabody communication system," IEEE Trans. Ind. Electron., vol. 58, no. 2, pp. 690-699, Feb. 2011. 
[68] T. G. Zimmerman, "Personal area networks (PAN): Near-field intra-body communication," Master's thesis, Media Lab., Massachusetts Inst. Technol.,Cambridge, MA, USA, 1995.

[69] K. Partridge, B. Dahlquist, A. Veiseh, A. Cain, A. Foreman, J. Goldberg, and G. Borriello, "Empirical measurements of intra-body communication performance under varied physical configurations," in User Interface Softw. Technol Symp., Nov. 2001, pp. 183-190.

[70] S.-J. Song, N. Cho, and H.-J. Yoo, "A 0.2-mW 2-Mb/s digital transceiver based on wideband signaling for human body communications," IEEE J. Solid-State Circuits, vol. 42, no. 9, pp. 2021-2033, Sep. 2007.

[71] M. S. Wegmueller, N. Felber, W. Fichtner, A. Lehner, O. Hess, J. Froehlich, N. Kuster, R. Reutemann, and M. Oberle, "Measurement system for the characterization of the human body as a communication channel at low frequency," in IEEE 27th Annu. Int. Conf. EMBC, Sep. 2005, pp. 3502-3505.

[72] M. S. Wegmueller, A. Kuhn, J. Froehlich, M. Oberle, N. Felber, N. Kuster, and W. Fichtner, "An attempt to model the human body as a communication channel," IEEE Trans. Biomed. Eng., vol. 54, no. 10, pp. 1851-1857, Oct. 2007.

[73] N. Cho, J. Bae, and H.-J. Yoo, "A $10.8 \mathrm{~mW}$ body channel communication/mics dual-band transceiver for a unified body sensor network controller," IEEE J. Solid-State Circuits, vol. 44, no. 12, pp. 3459-3468, Dec. 2009. 
[74] K. Al-Ashmouny, C. Boldt, J. Ferquson, A. Erdman, A. Redish, and E. Yoon, "Ibcom (intra-brain communication) microsystem: wireless transmission of neural signals within the brain," in IEEE 31th Annu. Int. Conf. EMBC, Sep. 2009, pp. 2054-2057.

[75] K. Sasagawa, T. Matsuda, P. Davis, B. Zhang, and et al., "Wireless intrabrain communication for image transmission through mouse brain," in IEEE 33th Annu. Int. Conf. EMBC, Sep. 2011, pp. 2917-2920.

[76] M. Sneve, N. Emaus, R. M. Joakimsen, and R. Jorde, "The association between serum parathyroid hormone and bone mineral density, and the impact of smoking: the troms stud," European Journal of Endocrinology, vol. 158, no. 3, pp. 401-409, 2008.

[77] Physiotherapy for Elbow Injuries, [Online] Available: http://iphysioperth.com. au/news/physiotherapy-for-elbow-injuries/, 2014.

[78] Z. Lucev, I. Krois, and M. Cifrek, "A capacitive intrabody communication channel from $100 \mathrm{kHz}$ to $100 \mathrm{MHz}, "$ IEEE Trans. Instrum. Meas., vol. 61, no. 12, pp. 3280-3289, Dec. 2012.

[79] J. J. Carr and J. M. Brown, Introduction to Biomedical Equipment Technology. New Jersey: Prentice Hall, 2000.

[80] Z. Lucev, I. Krois, and M. Cifrek, "Intrabody communication in biotelemetry," in Wearable and Autonomous Biomedical Devices and Systems for Smart Environment, A. Lay-Ekuakille, Ed. Springer, 2010. 
[81] T. I. Oh, S. Yoon, T. E. Kim, H. Wi, K. J. Kim, E. J. Woo, and R. J. Sadleir, "Nanofiber web textile dry electrodes for long-term biopotential recording," IEEE Trans. Biomed. Circuits Syst., vol. 7, no. 2, pp. 204-211, Apr. 2013.

[82] R. Sorrentino and G. Bianchi, Microwave and RF Engineering. Singapore: John Wiley and Sons, 2010.

[83] A. Sasaki, T. Mizota, H. Morimura, S. Yabe, and et al., "Extended noisychannel models for capacitively coupled personal area network under influence of a wall," IEEE Trans. Antennas Propag., vol. 62, no. 5, pp. 2802-2812, May 2014 .

[84] International Commission on Non-Ionizing Radiation Protection (ICNIRP), "Guidance for limiting exposure to time-varying electric, magnetic, and electromagnetic fields (up to $300 \mathrm{GHz}$ )," Health Physics, vol. 74, no. 4, pp. 494-522, Apr. 1998.

[85] World Health Organization, Electromagnetic Fields (300 Hz to $300 \mathrm{GHz}$ ), [Online] Available: http://www.inchem.org/documents/ehc/ehc/ehc184.htm, 1993.

[86] D. T. Nguyen, R. Kosobrodov, M. A. Barry, W. Chik, and et al., "Electrodeskin contact impedance: in vivo measurements on an ovine model," in Journal of Physics: Conference Series 434, 2013, pp. 1-4.

[87] A. M. Hayar and G. M. Vitetta, "Channel models for ultra-wideband communications: an overview," in 15th Mobile and Communications Summit, Jun. 2005, pp. $1-5$. 
[88] L. Liu, R. D.'Errico, L. Ouvry, P. De Doncker, and C. Oestges, "Dynamic channel modeling at $2.4 \mathrm{GHz}$ for on-body area networks," Advances in Electronics Telecommunications-Radio Communication Series: Recent Advances in Wireless Communication Networks, vol. 2, no. 4, pp. 18-27, 2011.

[89] D. Terfera and S. Jegtvig, Clinical Anatomy for Dummies. Hoboken, NJ : Wiley Press, 2012.

[90] Y. Song, K. Zhang, Q. Hao, and J. P. Rolland, "Modeling and characterization of the electrostatic coupling intra-body communication based on Mach-Zehnder electro-optical modulation," Optics Express, vol. 20, no. 12, pp. 13488-13500, Jun. 2012.

[91] [Online] Available: www.minicircuits.com/MCLStore/ModelInfoDisplay.,2013.

[92] J. Brown and B. Whitlock, "Common-mode to differential-mode conversion in shielded twisted-pair cables (shield-current-induced noise)," in Audio Engineering Society Convention 114. Audio Engineering Society, Mar. 2003, pp. 1-22.

[93] S. J. Mathes and F. Nahai, Reconstructive Surgery: Principles, Anatomy \& Technique, Volume 1. UK : Churchill Livingstone, 1997.

[94] Calculation of the Dielectric Properties of Body Tissues in the frequency range $10 \mathrm{~Hz}$ - $100 \mathrm{GHz}$, [Online] Available: http://niremf.ifac.cnr.it/tissprop/ htmlclie/htmlclie.htm\#atsftag, 2010.

[95] F. S. Lee and A. P. Chandrakasan, "A 2.5 nJ/b $0.65 \mathrm{~V}$ pulsed UWB receiver in 90 nm CMOS," IEEE J. Solid-State Circuits, vol. 42, no. 12, pp. 2851-2859, Dec. 2007. 
[96] A. Wong, M. Dawkins, G. Devita, N. Kasparidis, and et al., "A 1 V 5 mA multimode IEEE 802.15.6/bluetooth low-energy WBAN transceiver for biotelemetry applications," IEEE J. Solid-State Circuits, vol. 48, no. 1, pp. 186-198, Jan. 2013.

[97] M. S. Wegmueller, W. Fichtner, M. Oberle, and N. Kuster, "BPSK \& QPSK modulated data communication for biomedical monitoring sensor network," in IEEE 28th Annu. Int. Conf. EMBC, Sep. 2006, pp. 2071-2074.

[98] P. Harikumar, M. Kazim, and J. Wikner, "An analog receiver front-end for capacitive body-coupled communication," in IEEE 30th NORCHIP, Dec. 2012, pp. 1-4.

[99] H. Lee, K. Lee, S. Hong, K. Song, and et al., "A 5.5mW IEEE 802.15.6 wireless body area network standard transceiver for multi-channel electro-acupuncture application," in IEEE Int. Solid-State Circuits Conf., Feb. 2013, pp. 452-454.

[100] H. Sjoland, J. B. Anderson, C. Bryant, R. Chandra, and et al., "A receiver architecture for devices in wireless body area networks," IEEE J. Emerging Selected Topics in Circuits Syst., vol. 2, no. 1, pp. 85-95, Mar. 2012.

[101] D. Anzai, Y. Takagi, K. Katsu, and J. Wang, "Effect of energy detection duration on UWB-IR transmission performance in wireless body area networks," in IEEE 6th Asia-Pacific Conf. Environmental Electromagnetics (CEEM), Nov. 2012, pp. 218-221.

[102] S. Drago, D. Leenaerts, F. Sebastiano, L. Breems, K. Makinawa, and B. Nauta, “A $2.4 \mathrm{GHz} 830 \mathrm{pJ} /$ bit duty-cycled waked-up receiver with -82 dBm sensitivity 
for crystal-less wireless sensor nodes," in IEEE Int. Solid-State Circuits Conf., Feb. 2010, pp. 224-225.

[103] [Online] Available: www.xilinx.com, 2014.

[104] A. N. Kim, P. A. Floor, T. A. Ramstad, and I. Balasingham, "Communication using ultra wide-band pulse position modulation for in-body sensors," in IEEE/ACM rth BODYNETS Conf., Sep. 2012, pp. 159-165.

[105] S. H. Song and Q. T. Zhang, "CDMA-PPM for UWB impulse radio," IEEE Trans. Veh. Technol., vol. 57, no. 2, pp. 1011-1020, Mar. 2008.

[106] J. Wen Li, X. Chen, S. Hang Pun, P. Mak, and et al., "Bit error rate estimation for galvanic type intra-body communication using experimental eye-diagram and jitter characteristics," in IEEE 35th Annu. Int. Conf. EMBC, Jul. 2013, pp. 5195-5198.

[107] K. Shikada and J. Wang, "Development of human body communication transceiver based on impulse radio scheme," in IEEE 2nd CPMT symp., Dec. 2012, pp. 1-4.

[108] L. Yan, J. Bae, and H.-J. Yoo, "A 1 Mb/s, -75 dBm sensitive fully integrated body channel transceiver for a low energy compact wearable healthcare sensor," in IEEE Asian Solid-State Circuits Conf., Nov. 2010, pp. 1-4. 


\section{Appendix A}

\section{RTL Schematic}

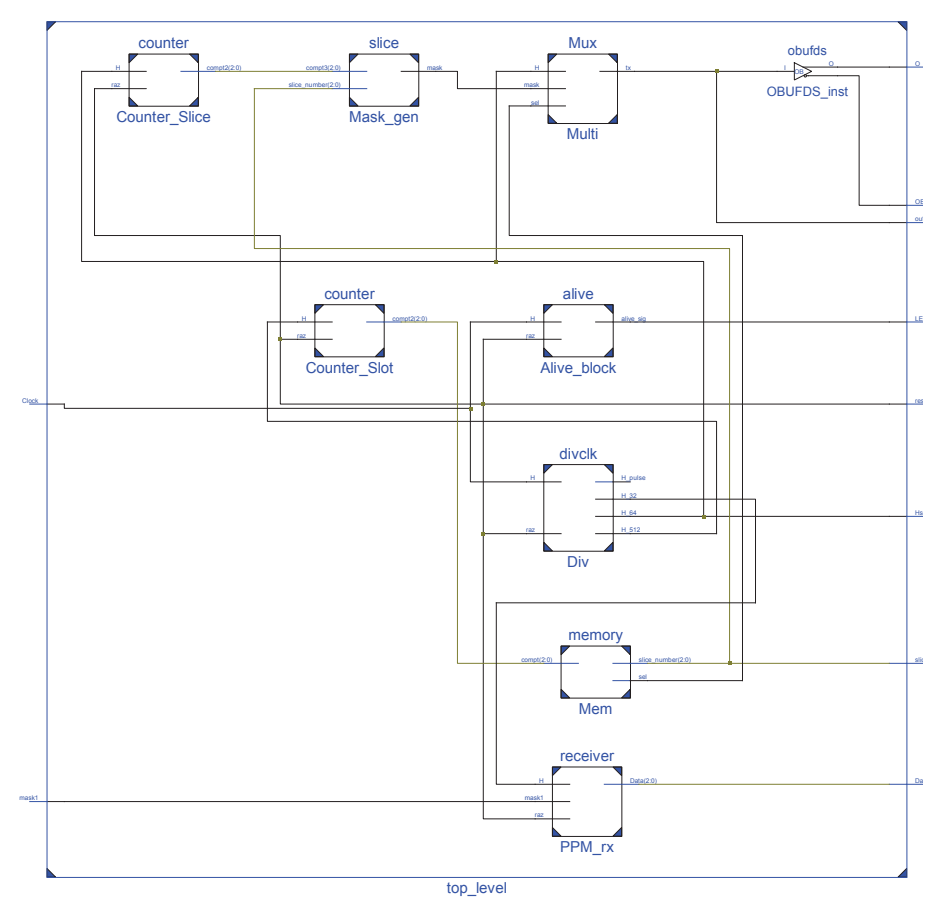

Figure A.1: The top-level register-transfer level (RTL) schematic of the proposed IBC tranceiver in this syudy. 